\title{
Molecular Structures of Gas-Phase Polyatomic Molecules \\ Determined by Spectroscopic Methods
}

\author{
Marlin D. Harmony \\ Department of Chemistry, The Unitersity of Kansas, Lawrence, KS 66045 \\ Victor W. Laurie \\ Department of Chemistry, Princeton University, Princeton, NJ 08540 \\ Robert L. Kuczkowski \\ Department of Chemistry, University of Michigan, Ann Arbor, MI 48109
}

R. H. Schwendeman

Department of Chemistry, Michigan State University, East Lansing, MI 48824

D. A. Ramsay

Herzberg Institute of Astrophysics, National Research Council of Canada, Otan:a, KIA ORo, Canuda

Frank J. Lavas, Walter J. Lafferty, and Arthur G. Maki

National Bureau of Standurds, TFrashington, DC 20334

\begin{abstract}
Spectroscopic data related to the structures of polyatomic molecules in the gas phase have been reviewed, critically evaluated, and compiled. All reported bond distances and angles have been classified as equilibrium $\left(r_{\mathrm{a}}\right)$, average $\left(r_{\mathrm{z}}\right)$, substitution $\left(r_{\mathrm{s}}\right)$, or effective $\left(r_{0}\right)$ parameters, and have been given a quality rating which is a measure of the parameter uncertainty. The surveyed literature includes work from all of the areas of gas-phase spectroscopy from which precise quantitative structural information can be derived. Introductory material includes definitions of the various types of parameters and a description of the evaluation procedure.
\end{abstract}

Key words: Bond angles; bond distances; gas-phase polyatomic molecules; gas-phase spectroscopy; microwave spectroscopy; molecular conformation; molecular spectroscopy; molecular structure; molecules; sirucrure.

\section{Contents}

1. Introduction. .................... 619

2. Definitions of Structural Parameters....... 620

3. Uncertainties. . . . . . . . . . . . . . . . 623

4. Evaluation Procedure... . . . . . . . . . . 625

5. Description of Tables... . . . . . . . . . . 625 Appendix: Selected Diatomic Molecule Distances. . 627 Structural Data Tables. . . . . . . . . . . . . . . . 628

\section{Introduction}

Since the first reports of high resolution microwave spectra more than thirty years ago, the microwave spectra of hundreds of molecules have been examined. By far the most common aim of these studies has been the determina. tion of geometrical structural parameters-internuclear

(C) 1979 by the U.S. Secretary of Commerce on behaif of the United States. This copyright is assigned to the American Institute of Physics and the American Chemical, Society.
Page

Inorganic Molecules. . . . . . . . . . . . . . . . . 628

$\mathrm{C}_{1}$ Molecules . . . . . . . . . . . . . . . . . 650

$\mathrm{C}_{2}$ Molecules. . . . . . . . . . . . . . . . . . 668

$\mathrm{C}_{3}$ Molecules. . . . . . . . . . . . . . . . . . . 688

$\mathrm{C}_{4}$ Molecules. . . . . . . . . . . . . . . . 702

$\mathrm{C}_{5}-\mathrm{C}_{11}$ Molecules................ 711

distances and angles and nuclear conformations-by analysis of molecular rotational constants. At the same time, precision uv-visible, infrared, and Raman spectroscopy have yiclded valuable structural results on relatively small molecules, and more recently molecular beam reasonance methods have played a small but important role. This report is a critical evaluation and compilation of gas-phase structural data for polyatomic molecules determined by these lechniques. The literature surveyed in this work covers largely the time period from the late 1940 's through 1976 and approximately half-way into 1977. 


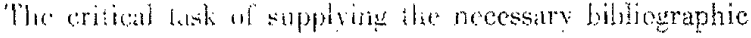
support lias besn performed by the Vational Bureau of Standurds, although the Iondolt-Börnstein tables $[1,2]^{1}$ have also been usefil in locating pertiment literature. We have tried to include in this survey all polyatomic molectiles for which reliable structural data are available for the ground electronic state from spectroscopic studies. Omissions have surely occurred, but it is hoped that they have been held to a minimum.

In the early stages of this project, it had been planned to irclude diatomic molecules as well as polyatomics. Sub. sequently, several factors led to the omission of this phase of the evaluation. First, Lovas and Tiemann [3j have recently published a critical review of the spectral data of all those diatomic molecules siludied by microwaye techniques up to 1974. Secondly, Herzberg and Huber [4] have near completion a comprehensive review of diatomic molecules which will up-to-date the spectral and molecular properties presented in the earlier tables of Herzberg [5]. Since diatomic molecules are of fundamental importiusce as the cornerstone of structural chenistry, and often provide an important point of comparison for polyatomic molecules, a selection of diatomic distances has been included in an Appendix to this work. In order than nearly all microwave structures might be available in a single source, the Appendix includes all those molecules review by Lovas and Tiemann [3]. Other molecules have hecn selected for inclusion primarily on the basis of their role as prototypes for the structural units of polyatomic molecules.

Previous compilations containing gas-phase structurit data of polyatornic molecules include the tables by sullon [6-, Appendix VIII of Gordy and Cook [?], and Appesuix VI of Heraberg [8]. The most recent compilation is that of Callomon et at. [2] covering gats-phase spectroscupic and diffraction data. For diatomic molecules, in addition to the sources mentioned in the previous paragraph, struclura] data have been compiled by Rosen [9] and have also appeared in the JANAF' 'Thermochemical 'lables [10].

The primary aim of the present work is the critical evaluation and compilation of reliable structural data according to a set of relatively well-defined guidelines. The evaluation procedure is described in more detail in subsequent sections, but in general it results in the inclusion of only those strtictural resulis that are reliably determined by experimental daia and are free of assumptions, intuition, and analogy. For the most part, this mears that only those polyatomic molecules for which more than one isotopic species has been studied are included. Exceptions include. cases for which unambiguous and non-trivial conformational information could be obtained from a study of only one species.

A second important aspect of the work is that every distance or angle reported has been classified according to a consistent set of operational definitions of the methods of analysis by which the parameters were oblained. These definilions $\left(r_{a}, r_{0}\right.$, etc.) are described in detail in the

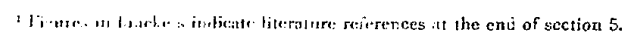

1. Pley: Clewm, Ref Dotca, Vai. 8, No. 3, 1979 following section. Unccrainties in the reported flaramelers have been reassessed by the reviewers and reported according $\mathrm{t}$ a consistent scheme. Finally, when it appeared to be necessary, distances and angles bave been recalculated from the reported rotational constants or from the spectra. Because there are many possible slight variations in methods of analysis, reported parameters were replaced by recalculated ones only when the two sets differed by amounts outside the estimated uncertainties.

The formal for the presentation of the structural data has been chosen for clarity and simplicity. Distances have bcen reported in $\AA$ ( $100 \mathrm{pm}$ ) units with no more than three decimal digits. This choice is a result of the fact that, except for a relatively small number of polyatomic moleculss, experimental and model errors lead to physically meanizgless numbers beyond $0.001 \AA$. For angles, reported in degrees, only one decimal digit has been listed. Param. ela $\mathrm{r}$ uncerlainlies have been indicated by a letter rating $(A, B \ldots)$ which defines a range of uncertainty. This scheme was chosen not only for simplicity and clarity, but also to discourage users from placing an unrealistic emphasis upon minor variations in parameters, or an undue reliausce upon a precise ancertainty. As described below, the uncertanties in structural parameters for the majority of polyatomic molecules are dominated by rotation-vibration interactions which can be estimated only within certain narrow ranges.

In the next section the various operational definitions of distances and angles are given. Following this, the method of assessing uncertainties is described, and a discussion of the gencral evaluation procedures is presented. As a final prologuc, a brief description is given of the format, content, and organization of the labulated data.

\section{Definitions of Structural Parameters}

Tho Hamiltonian used to analyze the rotational spectra of most molenules consists of some collection of the following terms:

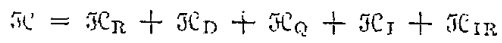

in which $x_{n}$ is the rigid rotator Hamiltonian, $\mathfrak{X}_{D}$ is the contribution: from ontrifligal distortion, $x_{Q}$ describes the nuclear quadrupole inleraction, $\tau_{I}$ contains the internal rotation energs, and $x_{1 \mathrm{n}}$ describes the interaction of internal and overall rolation. For purposes of distance and angle delermination the most important part of $(1)$ is $\mathfrak{T C}_{R}$;

$$
\pi_{\mathrm{H}}=h\left(A J_{u^{2}}+B J_{t}^{2}+C J_{c}^{2}\right)
$$

in which $J_{u}, J_{i}$ and $J_{s}$ are components of the rotational anguiar momentum in the directions of the molecule-fixed $a, b$, and $c$ principal inertial axes, and $A, B$, and $C$ are rotational constants. The forms of $\mathfrak{H}_{\mathrm{D}}, \mathcal{K}_{\mathrm{Q}}, \mathfrak{K}_{\mathrm{I}}, \mathfrak{K}_{\mathrm{In}}$, and any other possible contributions to the Hamiltonian will not be discussed here; it will be assumed that they hate been properly accounted for in each study. Where there 
was question as to whether this was in fact the case, the reviewer either reanalyzed the spectra or omitted the molecule from the compilation.

The rotational constante depend on the vibrational state of the molecules. For molecules for which all the vibrations are harmonic or nearly so, the dependence on vibrational state quantum numbers is as follows:

$$
A_{v}=A_{0}-\sum_{k} \alpha_{k}^{(A)}\left(v_{k}-d_{k} / 2\right)
$$

where $\alpha_{k}^{(A)}$ is a vibration-rotation parameter, and $v_{k}$ and $d_{k}$. are the vibrational quantum number and the degeneracy of the $k$ th vibrational mode, respectively; $"$ stands for $v_{1}, v_{2} \ldots, v_{3 N-6}$. In eq (3) $A$, is the $A$ rotational constant for the hypothetical equilibrium configuration of the molecule; that is,

$$
A_{\mathrm{p}}=h / 8 \pi^{2} I_{u}{ }^{(\mathrm{e})}
$$

where $l_{a}(e)$ is the moment of inertia of the molecule about the $a$-axis when all of the atoms are at equilibrium. Expressions similar to eq (3) and (4) may be written for $B_{v}, B_{c}, C_{n}$, and $C_{c}$. For any vibrational state $v$,

$$
A_{u}=h / 8 \pi^{2} I_{a}^{(v)}
$$

in which $I_{n t}{ }^{(v)}$ is an "effective" moment of inertia about the $a$-axis for molecules in the vibrational state $v$. Since in any sample most molecules are in the ground vibrational state $v=0$, the most commonly derermined rotational constants are $A_{0}, B_{o s}$ and $C_{0}$; the corresponding effective moments of inertia are $I_{a}^{(0)}, I_{b}^{(0)}$, and $I_{c}{ }^{(0)}$.

The principal moments of inertia may be defined in terms of the eigenvalues of an inertial tensor $\mathbf{P}$ with components defined as follows:

$$
\mathbf{P}_{\alpha \beta}=\sum_{i=1}^{N} m_{i} \alpha_{i} \beta_{i}
$$

in which $\alpha_{1}$ and $\beta_{i}$ are Cartesian $x, y$, or $z$ coordinates of an atom $i$ of mass $m_{i}$. The coordinates are measured from the center of mass of the molecule and the sum is over all the atoms. The principal second moments, taken in the order $P_{a a} \geq P_{b b} \geq P_{c c}$, are the eigenvalues of the tensor $\mathbf{P}$ and are related to the principal moments of inertia, as follows:

$$
\begin{aligned}
& I_{a}=P_{b b}+P_{a c}, \\
& I_{b}=P_{a a}+P_{c c}, \\
& I_{c}=P_{a a}+P_{b b} .
\end{aligned}
$$

From the definitions in eq (6.) and (7) it is clear that the coordinates of the atoms determine the principal moments of inertia. To the extent that the reverse is true-that the moments of inertia determine the coordinates of the atoms-it is possible to calculate distances and angles from experimentally-determined rotational constants.

There are two serious problems in determination of structural parameters from rotational constants. First, there are nearly always many bond distances and angles to be determined and there are only three rotational constants for a single molecular species. It is therefore usually necessary to determine rotational constants for molecules with differing isotopic composition of the nuclei. To high approximation the equilibrium structures of molecules are invariant to isotopic substitution. Thus, equilibrium bond distances and angles, so-called $r_{\mathrm{c}}$ parameters, are determined by adjuting the parametors to give calculated moments of inertia which agree with experimentally. derived equilibrium moments for as many isotopically. substituted species as are necessary.

The second serious problem in structure determination is that it is seldom possible to obtain equilibrium moments of inertia from the spectra. In the most common situation effective rotational constants for the ground vibrational states of one or more isotopic species are available. In addition to the sccond moment contributions from the sums over the atomic coordinates. effective moments of inertia contain contributions from averaging over the zero-point vibrations and from other vibration-rotation interactions (mainly Coriolis effects). These extra contributions are mass dependent so that the assumption of invariance of structural parameters to isotopic substitution is not valid. Parameters determined by adjusting bond distances and bond angles to give calculated moments of inertia which best fit experimentally derived effective moments of inertia are called effective parameters or $r_{0}$ parameters.

In general, equilibrium bond distances and angles determined from different sets of moments of inertia are found to agree within the uncertainties propagated by the experimental uncertainties in the moments. By contrast, effective distances and angles are nearly always strongly dependent on the particular set of moments of inertia used to obtain them. Furthermore, the variations in parameters from set to set may be an order of magnitude or more larger than the propagated experimental uncertainties. As a consequence, uncertainties in effective parameters cannot be deduced from the uncertainties which commonly accompany the results of a least-squares fitting routine. The method used for estimating the uncertainties for effective parameters in the present compilation is described in the next section.

It is possible to reduce the effect of vibration-rotation interactions on the determination of structural parameters frous effective unuments of inertia by fitling differences in corresponding moments for different isotopic species rather than fitting the moments themselves. This technique is based on the assumption that the vibration-rotation contributions to the moments are very similar for two isotopic species. Since they are mass dependent, however, there is only partial cancellation, so it should not be surprising to find that when the differences in moments of inertia for two isotopic species are very small, the residual differences in vibration-rotation contributions become important. 
Differences in moments of inertia may be fit directly by least-squares procedures just as may be done for the momen's themsclves. However, the simplest procedure is to make use of Kraitchmar's equations [I1], which are specifically for the case in which two isotopic species of the same molecule differ in isotopic labeling at a single atomic center. One molecule, usually the most common jsotopic species, is designated as the parent molecule. Then, according to Kraitchman's equations, the $a$ coordinate of the atom which is isotopically labeled in the substituted molecule is given by the expression [11]

$$
\begin{array}{r}
u_{\mathrm{s}}=\left\{\left(\Delta P_{a a} / \mu\right)\left[I+\Delta P_{b b} !\left(P_{b b}-P_{a a}\right)\right]\right. \\
\left.\left[1+\Delta P_{c c:}\left(P_{c c}-P_{a a}\right)\right]\right\}^{1 / 2}
\end{array}
$$

In eq (8) $P_{a a}, P_{b b}$ and $P_{a c}$ are effective principal second moments of the parent molecule and

$$
\mu=M_{\mathrm{p}} \Delta \mathrm{m} /\left(M_{\mathrm{p}}+\Delta \mathrm{m}\right)
$$

in which $M_{p}$ is the molecular mass of the parent molecule and $\Delta m$ is the change in mass of the isotopically-sub. stituted atom. Also,

$$
\Delta P_{n \theta}=P_{e o}^{\prime}-P_{p o}
$$

for $g=a, b, c$, in which $P^{\prime}{ }_{\theta 0}$ is an effective principal second moment of the isotopically substituted molecule. Expressions similar to eq (8) may be written for $b_{s}$ and $c_{\mathrm{s}}$ by cyclic permutation of subseripts. If the effective moments of inertia are determined for a parent molecule and two singly-substituted species, the coordinates of two atoms may be determined and from them the distance between the atoms. From the data for a parent and three singly-substituted species an interatomic angle can be calculated, etc. Parameters determined by means of Kraitchman's equations are called substitution parameters or $r_{s}$ parameters [12]. In many structure determinations some of the parameters have been determined by Kraitchman's equations, whereas others are determined by least squares adjustment. In this compilation the former are always labeled $r_{s}$ parameters. Parameters determined by least squares adjustment to fit only differences in moments of inertia are considered to be $r_{s}$ parameters also. If, however, any moments of inertia or second moments of inertia are used to determine coordinates of an atom on which no substitution was made, parameters obtained from those coordinates are labeled generally as $r_{0}$.

In addition to eq (8) and the corresponding equations for $b_{\mathrm{s}}$ and $c_{\mathrm{s}}$, it is possible to derive similar equations for multiple substitution at symmetrically equivalent sites [15-15]. Parameters determined from coordinates obtained by these equations are labeled as $r_{\mathrm{s}}$. It is also possible to derive special forms of the Kraitchman equations for coordinates of atoms which lie in a plane of symmetry or on a symmetry axis $[11,7]$. Because a symmetry plane is also a principal inertial plane, the change in the second moment perpendicular to such a plane should vanish for isotopic substitution of an atom in the plane: For substitution on a symmetry axis the changes in both second moments perpendicular to the axis should vanish. For example, for isotopic substitution in the $a b$ inertial plane, $\Delta P_{c c}$ should be zero so that $\Delta I_{a}+\Delta I_{b}$ should equal $\Delta I_{c}$. Under these circumstances three combinations of the principal moments of inertia could be used to calculate $\Delta P_{a u}$, as follows:

$$
\begin{aligned}
& \Delta P_{a a}=\left(\Delta I_{b}+\Delta I_{\mathrm{c}}-\Delta I_{a}\right) / 2 \\
& \Delta P_{a a}=\Delta I_{b} \\
& \Delta P_{a a}=\Delta I_{c}-\Delta I_{a}
\end{aligned}
$$

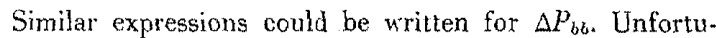
nately, the vibration-rotation contributions do not completely cancel so that $\Delta P_{\mathrm{cc}}$, as calculated from effective moments, is never exactly zesu for substitution iss llse $u b$ plane. As a result, the substitution coordinates depend on which of eqs (11) is used to compute $\Delta P_{a \text { at. Since no one of }}$ these expressions is theoretically preferred, all of the possible combinations should be tested to insure that the parameters reported are representative. Since this has not always been done, the reviewers looked for the possibility of unrepresentative use of Kraitchman's equations. A similar, but more serious problem occurs in the determination of substitution coordinates for molecules which are near oblate symmetric tops. For near oblate tops, $P_{u a}$ is approxinatcly equal to $P_{b b}$ so that the effecl of $\Delta l_{b b}$ in the second factor of eq $(8)$ is greatly magnified. In the corresponding equation for $b_{s}$ the effect of $\Delta P_{c o}$ is magnified. If either $\Delta P_{t h}$ or $\Delta P_{c c}$ is small enough that vibration-rotation contributions are a significant fraction of the value, sizable uncertainties in $a_{s}$ or $b_{s}$ will result. A procedure for treating this situation by use of data from additional isotopically-labeled species has been described [16].

Examination of the form of eq (8) reveals that the effect of isotopic substitution on the moments of inertia decreases rapidly as the substitution site approaches an inertial plane. This is most easily seen by calculating the change in a coordinate which results from changes in the $\Delta P_{\text {go }}$. In most cases the factors in square brackets in eq $(8)$ contribute an order of magnitude or so less to this change than the first factor. (An exception is the near oblate tops just mentioned.) With these contributions ignored it is found that

$$
\delta a_{s}=\delta \Delta P_{u a} / 2 \mu a_{s *}
$$

Similar expressions may be derived for $\delta b_{s}$ and $\delta c_{s,}$ Contri butions to $\delta \Delta P_{u a}$ come from experimental uncertainty in the $P_{a n}$ values and from mass-dependent vibration-rotation interactions. It is clear from eq (12) that as $a_{s} \rightarrow 0, \delta a_{\mathrm{s}}$ gets very large so that the determination of $a_{s}$ by Kraitchman's equations becomes questionable. If only one coordinate is close to a given plane, it may be determined 
by the appropriate first moment relation

$$
\sum_{i=1}^{N} m_{i} g_{i}=0 ; g_{i}=a_{i}, b_{i} \text {, or } c_{i}
$$

Substitution coordinates are known to satisfy the first moment relations to a good approximation. It is also possible to determine coordinates for atoms close to an inertial plane by the double substitution method of Pierce [17]. In this report coordinates determined either by eq (13) with all $g_{i}$ but one being substitution coordinates or by Pierce"s method are referred to as $r_{\mathrm{s}}$ coordinates.

Subsitution parameters were shown by Costain [12] to be considerably less sensitive than effective parameters to the particular set of isotopic species used to determine them. In addition, it is believed that the equilibrium structure is more closely approximated by the $r_{\mathrm{s}}$ structure than by the $r_{0}$ structure. For example, Costain [12] has shown that $r_{\mathrm{s}} \simeq \frac{3}{2}\left(r_{\mathrm{o}}+r_{\mathrm{c}}\right)$ for diatomic molecules.

Watson [18] has defined an $l^{(\mathrm{m})}$ moment of inertia as follows:

$$
I_{n}^{(m)}=2 I_{n}^{(s)}-I_{n}^{(0)}
$$

in which $I_{f(}{ }^{(g)}$ is the moment of inertia about the $g$-axis calculated from the $r_{*}$ parameters. The $I^{(\mathrm{m})}$ moments were shown to be equal to equilibrium moments of inertia to first order. Watson [18] has devised a procedure for determining structural parameters from $I^{(\mathrm{m})}$ moments. The resulting bond distances and bond angles are designated as $r_{\mathrm{m}}$ parameters. The $r_{\mathrm{m}}$ procedure is difficult to apply in general because data of very high precision are required for a large number of isotopic species. In addition, the approximation that $I^{(\mathrm{m})} \sim I^{(\mathrm{e})}$ breaks down for very light atoms, so that $\mathrm{H}$-atom parameters cannot be determined by this method. Since the $r_{\mathrm{rn}}$ method has been applied to very few molecules, parameters of this 1 ypo has not been included in this compilation.

Oka [19], Herschbach and Laurie [20], and Toyama, et al. [2I], showed that the effective moments of inertia and the moments of inertia of the molecule with all of its atoms in their average positions are related by terms which depend only on the harmonic part of the vibrational potential function. Since the harmonic potential terms are known for many small molecules, a set of moments $I^{(2)}$ for the average configuration can be derived from $I^{(0)}$ values. The bond distances and bond angles derived from the $I^{(z)}$ moments for the ground vibrational state are called average parameters or $r_{z}$ parameters. Average parameters are mass dependent so that when more than three independent structural paramelers must be determined, isotope effects must be estimated. These are typically estimated by assumption of an anharmonicity correction for bond stretching and from computed vibrational amplitudes [22]. In this compilation, no distinction is made between average parameters determined from $I^{(s)}$ moments for a single isotopic species and those determined from $I^{(z)}$ moments for several species with isotope corrections.

\begin{tabular}{|c|c|}
\hline Parameter & Defmition \\
\hline$r_{\mathrm{\theta}}$ (Equilibrium) & $\begin{array}{l}\text { Distance or angle between equilibrium nuclear } \\
\text { positions. }\end{array}$ \\
\hline$r_{z}$ (Average) & $\begin{array}{l}\text { Distance or angle between average nuclear } \\
\text { positions in the ground vibrational state. }\end{array}$ \\
\hline$r_{\mathrm{s}}$ (Substitution) & $\begin{array}{l}\text { Distance or angle calculated from coordinates } \\
\text { determined by Kraitchman's equations. }\end{array}$ \\
\hline$r_{\mathrm{o}}$ (Effective) & $\begin{array}{l}\text { Distance or angle from coordinates adjusted to } \\
\text { give hest fit to effective ground state rotational } \\
\text { constants. }\end{array}$ \\
\hline$\tau_{\mathrm{m}}$ & $\begin{array}{l}\text { Approximate equilibrium distance or angle cal- } \\
\text { culated from coordinates determined by Wat- } \\
\text { son's equations. }\end{array}$ \\
\hline$r_{g}$ & Thermal average value of distance or angle. \\
\hline
\end{tabular}

TABLE 1. Definitions of structural parameters

It is important to distinguish between $r_{\mathrm{z}}$ parametersbond distances and angles for the atoms frozen in their average positions-and $r_{l /}$ parameters-the true ground vibrational state average values of distances and angles. The $r_{g}$ parameters are determined by electron diffraction. It was pointed out by Morino et al. $\lceil 237$ and by Kuchitsu [22] that the difference between an $r_{s}$ and an $r_{s}$ distance is the difference between averaging over the molecular motion before or after the distance is calculated. As shown by Kuchitsu, the $r_{z}$ distance is to good approximation the average of the projection of the distance on the line connecting the nuclei at equilibrium.

The different definitions of structural parameters are summarized in table 1 . Comparisons of the different parameters for representative molecules have been given in many places (e.g., in refs. 7, 12, 20, 24, 25, and 26). In addition, many such comparisons appear in data tabulated in this report. Examination of such comparisons shows that differences of several thousandths of an Angstrom unit in distance and several tenths of a degree in angle should be expected for the different definitions of a given parameter in a molecule. By contrast, the strictly experimental uncertainty is often less than $0.001 \AA$ or $0.1^{\circ}$. As a result, the definition of a reported structural parameter is an important consideration, particularly when parameters from different molecules are compared.

\section{Uncertainties}

In order to report uncertainties in structural parameters it is necessary to define the basis for the uncertainty estimate. For $r_{\infty}$ and $r_{z}$ parameters, which have well-defined mathematical and physical models, it is sensible to estimate the uncertainties strictly on the basis of the experimental uncertainties of the input data. On the other hand, for $r_{0}$ and $r_{*}$ parameters of polyatomic molecules the operational definitions lead to parameters which are less well-defined physically because of the effects of zero-point vibrationrotation interactions. In this case one would like to know the extent to which these generally unknown moleculedependent terms contribute to the computed value of the 
parameicrs. (hie way of assessing such contributions would be in compute the values of the paramelers $\left(r_{0}\right.$ or $\left.r_{s}\right)$ by using several different independent combinations of isotopic data, a procedure which normally leads to a range of values. This range could then serve as a measure of the uncertainty in the computed parameter.

A second way of assessing the uncertainly in $r_{\mathrm{o}}$ or $r_{\mathrm{s}}$ parameiers is to attompt to compute the extent to which vibration-rotation terms cause these parameters to differ from the $r_{e}$ value. Such a computation cannot be made precisely in the general case; if it could, the more desirable $r_{c}$ parameter would be obtainable. It is possible, however, to compute an estimate of the general magnilude of the vibration-rotation contributions. Such an approach is the one taken here; the result is an operational definition of the uncertainty which is described bolow by eqs $(20)-(22)$.

The derivation of eqs (20)-(22) follows from eq (12). For this derivation eq (7) is inverted to give

$$
P_{a a}^{(0)}=\left(I_{b}{ }^{(0)}+I_{c}^{(0)}-I_{a}^{(0)}\right), 2
$$

and

$$
P_{a c t}^{(s)}=\left(I_{b}^{(0)}+I_{c}^{(0)}-I_{a}^{(c)}\right) / 2 .
$$

Then, a pseudoinertia defect $\Delta_{\text {ra }}$ is defined to be

$$
\Delta_{a s}=2\left(P_{a a}^{(e)}-P_{a a}^{(0)}\right)
$$

from which it follows that, upon isotopic substilution,

$$
\begin{aligned}
& \left.\Delta P_{a a}{ }^{(0)}=P_{a a}{ }^{(0)}\right)-P_{u a}(0) \\
& =P_{a a}(\mathrm{e}) \prime-P_{t a n}(\mathrm{e})-\left(\Delta^{\prime}{ }_{4 a}-\Delta_{t a}\right) / 2 .
\end{aligned}
$$

Therefore, the vibration-rotation contribution to $\delta \Delta P_{n \text { an }}$ in eq (12) may be estimated as

$$
\delta \Delta P_{a n}=\left|\Delta_{a n}^{\prime}-\Delta_{n a}\right| / 2 .
$$

If the value $0.006 \mathrm{u} \cdot \AA^{2}$ is used for the difference in the pseudomertia defect upon isotopic substitution, it is found that

$$
\delta a_{s} / \AA= \pm\left|\frac{0.0015}{a_{s} ! \AA}\right| .
$$

A value of 1 u has been assumed for $\mu$. Equation (20) for the uncertainty in the coordinate of an atom derived by application of Kraitchman's equations is known as the Costain rule [27]. Although it has been derived for substiturion parameters, it expresses the general insensitivily of the moments of inertia of a molecule to isotopic substitution near a principal inertial plane. The Costain rule should therefore have wide application to the estima. tion of uncertainties in both $r_{\mathrm{s}}$ and $r_{\mathrm{o}}$ parameters.

The chnice of $0.006 u \cdot \AA^{2}$ for $\delta \Delta P_{t \prime}$ is based on an culimite of a lypical difference in pseudoinertial defect upon isolopic substitution. In some cases, parlicularly for $H$ coordinates, a larger value may be desirable, while for heary atoms the estimale may be somewhat conservative. The value may also be increased for cases in which the experimental uncertainty in $\Delta P_{y y}$ is an appreciable fraction of $0.006 \mathrm{u} \cdot \AA^{2}$ or in cases in which an unusully large range of coordinate values is obtained by using different combinations of coordinates in Kraitchman's equations.

The unambiguous conversion of uncertainties in atomic coordinales of the $r_{s}$ or $r_{\mathrm{n}}$ type in uncertainties in interatomic distances or angles requures more knowledge than is usually available. It is known that the use of the Kraitchman equations can lead to systematic vibration-rotation contributions to the uncertainties in the coordinates $[12,20]$. By contrast, in most cases it is probably safe to assume that the experimental contributions to the coordinate uncertainlies are distributed randomly. It is possible to compute uncertainties in inleratomic parameters by assuming that the contributions to the uncertainties in coordinates from experimental error are random and that the vibration-rotation contributions to each $\delta_{s},(g=a, b, c)$ have the same sign as $g_{\text {s }}$ [28]. However, the latter assumption is not always valid and the method requires that the effects of courdinate uncertaintes be added absolutely, which probm ably leads to overstatement of the uncertainties in the disiance and angles. In view of these ambiguities it was decided for the purposes of this compilation to use the usual formula for the propagalion of random uncertainties to calculate the uncertainties in distances or angles. For the distance between the ith, and $j$ th nuclei this leads to an uncertainty

$$
\delta r_{i j}=\left\{\sum_{i, a, b, \varepsilon}\left[\left(\frac{\partial r_{i j}}{\partial_{i ;}}\right)^{2} \delta g_{i}^{2}+\left(\frac{\partial r_{i j}}{\partial g_{j}}\right)^{2} \delta g_{j}^{2}\right]\right\}^{1 / 2} .
$$

In this equation $\left(\partial r_{i j}{ }^{\prime} \partial g_{i}\right)$ and $\left(\partial r_{i j}{ }^{\prime} \partial g_{j}\right\rangle$ are calculated from the final structure and $\delta g_{i}$ and $\delta g_{j}$ for $g=a, b, c$ are oblained by eq $(20)$ or as described in the next paragraph. $A$ corresponding equation for $i \theta_{i j k}$ is used to estimate the uncertainties in the angles.

Ar exception to the use of eq $(20)$ for the determination of the uncerlainly in an $r_{\mathrm{o}}$ or $r_{\mathrm{s}}$ coordinate is made for those coordinates which are calculated by the use of frest moment or second moment relations. For example, the uncertainty i1 a cuvilindie $g_{1}$ which has been calculated by eq (13) is obtained as follows:

$$
\delta g_{1}=m_{\perp}^{-1}\left[\sum_{i=2}^{N}\left(m_{i} \hat{o} g_{i}\right)^{2}\right]^{1 / 2}
$$

where the $\delta g_{i}$ for $i=2$ to $N$ arc obtained from eq (20).

As mentioned above, eqs $(20)-(22)$ provide the basic operational definition of the uncertainty in an $r_{\mathrm{s}}$ or $r_{\mathrm{o}}$ bond distance or angle which has been used throughout this rork. This definition is intended to be a combincd estimate of the contributions of experimental unccrtainty and vibration-rotation interaction. It should not, however, be taken too literally. For example, while it is expected that 
TABLE 2. Definition of letter symbols for uncertainties

\begin{tabular}{|c|c|c|}
\hline \multirow{2}{*}{ Symbol } & \multicolumn{2}{|c|}{ Uncertainty range } \\
\hline & Distance $/ \AA$ & Angle/degrees \\
\hline $\begin{array}{l}\mathrm{A} \\
\mathrm{B} \\
\mathrm{C} \\
\mathrm{D} \\
\mathrm{E} \\
\mathrm{X}\end{array}$ & $\begin{array}{l}\leq \pm 0.002 \\
\pm 0.002- \pm 0.005 \\
\pm 0.005- \pm 0.010 \\
\pm 0.010- \pm 0.020 \\
\pm 0.020- \pm 0.050 \\
\text { unable to evaluate } \\
\begin{array}{l}\text { unknown, or un- } \\
\text { reliable }\end{array}\end{array}$ & $\begin{array}{l}\leq \pm 0.2 \\
\pm 0.2- \pm 0.5 \\
\pm 0.5- \pm 1.0 \\
\pm 1.0 \quad \pm 2.0 \\
\pm 2.0- \pm 5.0\end{array}$ \\
\hline
\end{tabular}

the $r_{0}$ value of the distance or angle will lie within the uncertainty range in many cases, the approximations are such that this cannot be guarantecd.

Uncertainties in equilibrium and average parameters are assigned, as mentioned earlier, by estimating the uncertainties in the input data. The principal contributions to the uncertainty in equilibrium parameters is experimental uncertainties in the measured spectra. The bond distances and bond angles should be isotopically invariant to high approximation. Thus, standard least-squares methods of propagating the uncertainties in the rotational constants to uncertainties in the parameters can be used. The situation for uncertainties in average $\left(r_{z}\right)$ parameters is only slightly different. Here, the principal contributions to uncertainties in the moments of inertia come from experimental uncertainties and from uncertainties in the estimation of $I_{\theta}{ }^{(g)}$ from $I_{n}{ }^{(0)}$. However, additional contributions may come from estimation of isotope effects if moments of inertia from more than one species are required.

In this compilation the numerical values of the uncertainties in the distances or angles are not reported explicitly: a letter rating which mrresponds to a range of uncertainty is used instead. The correspondence between the letters used and the uncertainty ranges is given in table 2 . The selected ranges for the $A, B, C$.. ratings are, of course, arbitrary, but they seem to provide a useful classification of the accuracy of the numbers reported while at the same time they discourage a too-literal interpretation of the uncertainty estimates.

\section{Evaluation Procedure}

Although each reviewer adopted his own procedure for evaluating the structural parameters in the molecules assigned to him, some steps were common to all of the procedures. These are as follows:

1. The spectral fitting process was examined and uncertainties in the rotational constants were assessed.

2. The method of structural analysis used by the authors was checked for logic and evaluated in a general way. Different definitions. were assigned to each of the parameters according to the method of analysis (table 1 ).
3. For $r_{\mathrm{e}}$ and $r_{\mathrm{z}}$ parameters the errors were established by the errors in the experimental input data. Normally it was found that the published results had a proper assess: ment of the uncertainties. Numerical uncertainties were converted to letter ratings according to table 2 .

4. For $r_{\mathrm{o}}$ and $r_{\mathrm{s}}$ parameters, coordinate uncertainties were established by eq (20) or an equation such as eq (22). These were converted to parameter uncertainties using eq “(21) and to letter ratings via table 2. Special methods, such as the "double-substitution" procedure [17], were evaluated as individual cases. For $r_{0}$ structures, the range of values obtained by using differing data sets provided an. additional test of the parameter validity, normally leading to a rcduccd letter rating.

5. In most cases, structural results were not included if all ratings were $X$.

6. Studies which reported only conformational results were included when they were unambiguous and non-trivial. Although this represents the least objective aspect of the compilation, such results were deemed to be of sufficient interest and value to merit inclusion.

7. Because of the strong influence of vibration-rotation terms on parameters involving $\mathbf{H}$ atoms, such parameters have in most cases been rated no higher than $B$.

8. In general, the use of parameter assumptions was considered sufficient to invalidate a structure or to lead to $X$ ratings for the dependent parameters. However, in some well-defined castes the uncertainties in computed parameters were assessed according to the plausible uncertainties in the assumed parameters. Since $\mathrm{H}$ atom parameter assumptions have a relatively small influence upon heavy atom parameters, such assumptions were normally permitted.

9. The $\mathrm{E}$ rating has been used sparingly since a parameter with such a large uncertainty often has little practical validity. Thus a $\mathrm{CH}$ bond distance would usually be rated $X$ rather than $E$ in order to indicate its imprecise character. On the other hand, an $\mathrm{E}$ rating might provide a useful guideline for certain bond angles or for some of the more uncommon bond lengths.

10. Spot check calculations or complete recalculations of the structure were performed if any results seemed in doubt. Parameters in the original literature were replaced by recalculated values only if the values differed by more than the assigned uncertainty.

\section{Description of Tables}

Each mulecule in this compilation has been identified by its empirical formula according to the conventional scheme. The tables have been arranged so that the inorganic molecules appear first, followed in order by $\mathrm{C}_{1}, \mathrm{C}_{*} . \mathrm{C}_{3}$, etc. carbon-containing specics. Compound names generally follow the standard I.U.P.A.C. or Chemical Abstracts nomenclature schemes, but common names are often included also. Where the empirical formula alone is not sufficient for the identification of the structural parameters, a more conventional structural formula or drawing is included. Additional aid in interpreting the structures is 
provided by the conventional point group symbol, which is listed for each molecule above the data table.

Structural paramaters for each molceulc ure tabulated according to their operational definition, viz., substitution $\left(r_{3}\right)$, equilibrium $\left(r_{\mathrm{c}}\right)$, effective $\left(r_{0}\right)$, or average $\left(r_{z}\right)$, and are separated into distance and angle categories. As a general rule, $r_{\mu,}, r_{s}$, and $r_{z}$ parameters are always listed $w^{\text {then }}$ available; $r_{0}$ values are normally included only if one of the more reliable types can not be obtained from the experimental data. All distance values are in $\vec{A}(100 \mathrm{pm})$ units and angles are in degrees. Explanatory comments and footnotes follow the tabulated parameters, and finally the original literature sources are listed. In most cases only those sources have been referenced which are necessary to obtain the reported structure.

All structural data in this compilation refer to the ground electronic state, which for most polyatomic molecules implies a totally symmetrical orbital state with a spin multiplicity of unity. For those few molecules whose ground states do not fall in this category, the standard term symbol has been listed below the table. Lxeept for a few cases as noted, all structural parameters refer to the normal (most abundant) isotopic species, although the equilibrium parameters are expected to he isotopically invariant.

\section{Acknowledgments}

This work has been performed under the auspices and with the support of the National Bureau of Standards Office of Standard Reference Data. The overall coordinatiun, support, and encouragement of Dr. D. R. Lide has been much appreciated. Special thanks go to Gloria Rotter at the Bureau who handled the difficult task of literature searching and bibliographic support. Finally, the services of Nancy Murray, who typed most of the tables, and Norman Carpenter, who handled the artwork, have been much appreciated.

\section{References}

[1] Tandolt Rornctein, New Series, Group IL Volumos it and 6, Editor-in-Chief, K. H. Hellwege, Springer-Verlag, Berlin (1974).

[2] Callomon, J. H., Hirota, E., Kuchitsu, K., Lafferty, W. J.,
Maki, A. G., and Pote, C. S., "Structure Data of Free Poly. atomic Molecules," in Landolt-Bornstein, New Series, Croup 11. Volume 7, Editor-in Chief, K. H. Hellwege, Springer-Verlag, Berlir (1976).

[3] Lovas, F. J., and Tiemant, E., J. Phys. Chem. Ref. Data 3, 603 (1974).

[4] Huber, K. P., and Herzberg, G., Molecular Spcctra and Molecular Structure, Fol. $l V$. Constants of Diatomic Molecules, Van Vostrand Reinhold Company, New York (in nress).

[5] Heraberg, G., Spectra of Diatomic Molecules, D. van Nostrand Co., Ine., Prirceton, N. I. (1950).

[6] Sutton, L. E., Tables of Interatwaic Discences and Configuration in Itolecules and lons, The Chemical Society, Loncion (1958).

[7] Gordy. Wo and Cook, R. Lu, Microtrave Molecular Spectra, Interscience Publishers, New York, N. Y. (1970).

[8] Heraberg, $C_{n}$. Electronic Spectra and Eleotronio Structure of Polyatomic Molecules, D. van Nostrand Co., Inc., Princeton; N. J. $(1966)$,

[9] Rosen, B., Spectroscopic Data Relative to Diatomic Molecules," Pergamor Press, New York, N. Y. (1970).

[10] Stull, D. R., Prophet, H., et. al., JANAF Thermochemical Tables, 2nd Edition, NSRDS-NBS 37 (1971).

[11] Kraitchman, J., Amer, J. Phys. 21, 17 (1953).

[12] Costain, C. C., J. Chem. Phys. 29, 864 (1958).

13] Chutjian, A., J. Mol. Spectrose. 14, 361 (1964)

[14] Nygaard, Ln, J. Mol. Spectrosc. 62, 292 (1976).

[15] Li. Y. S., Kiser, K. L., and Durig, J. R., J. Mol. Spectrcsc. 42, $430(1972)$.

[lo] Kuczkowski, R. L., Gilies, C. W., and Gallaher, K. L., J. Mol. Spectrose. 60, 361 (1976).

[17 J Pierce, L., J. Mol. Spectrosc. 3, 575 (1959).

[18] Watson, J. K. G., J. Mol. Spectrosc. 48, 4.79 (1973).

[19] Oka, T., J. Phys. Soc. Japan 15, 2274. (1950).

[20] Herschbach, D. $R_{x}$, and Laurie, V. W. J. Chem Phys. 37, 1668 (1962); Ialurie, V. W., and Herschbach, D. R., J. Chem. Phys. 37, 1687 (1962).

[21] Toyama. M... Oka. T., and Morino. Y.. I. Mol. Speetrose. IB, $193(1964)$.

[22] Kuchitsu, K., and Cyvin, S. J., Molecular Structures and Vibrations, S. J. Cyvin, Ed., Flsevier, Amsterdam (2972), Chap. 12.

[23] Morino, Y., Kuchitsu, K., and Oka, T., J. Chem. Phys. 36, $1108(1962)$.

[24] Kuchitsu, K., J. Chem. Phys. 49, 4456 (1968).

[25] Lide, D. R., Jr., MTP International Review of Science, Physical Chemistry Series Two, A. D. Buckingham, Ed., Butterworths, London (1975), Vol. 2, Chap. 1:

[26] Schwendeman, R. H., "Critical Evaluation of Chemical and Physical Structural Information," D. R. Lide and M. A. Paul, Eds., National Academy of Sciences, Washington (1974), p. 24.

[27] Costain, C. C., Trans, Am. Cryst. Assoc. 2, 157 (1966).

[28.] Tobiason, F. L., and Schwendeman, R. H., J. Chem. Phys. 40, 1014 (1964). 
Appendix: Selected Diatomic Molecule Distances ${ }^{a}$

\begin{tabular}{|c|c|c|c|c|c|c|c|c|}
\hline Molecule & $r_{0}$ & $r_{\theta}$ & Molecule & $r_{o}$ & $x_{0}$ & Molecule & $r_{\mathrm{o}}$ & $r_{\theta}$ \\
\hline $\mathrm{AgBr}$ & 2.395 & 2.393 & $\mathrm{CiCs}$ & 2.910 & 2.906 & $\mathrm{HN}$ & 1.045 & 1.035 \\
\hline $\mathrm{AgCl}$ & 2.284 & 2.281 & $\mathrm{CIF}$ & 1.632 & 1.628 & $\mathrm{HO}$ & 0.980 & 0.971 \\
\hline $\mathrm{Ag} F$ & 1.987 & 1.983 & $\mathrm{CIGa}$ & 2.205 & 2.202 & $\mathrm{HP}$ & 1. $433^{*}$ & \\
\hline AgI & 2.547 & 2.545 & $\mathrm{ClH}$ & 1.284 & 1. 275 & HS & 1. 355 & $1.345^{*}$ \\
\hline$A i B r$ & 2.298 & 2.295 & $\mathrm{ClI}$ & 2.324 & 2.321 & $\mathrm{HSi}$ & 1.531 & $1.520^{*}$ \\
\hline AlCl & 2.133 & 2.130 & $\mathrm{ClIn}$ & 2.404 & 2.401 & $\mathrm{H}_{2}$ & 0.751 & $0.741^{*}$ \\
\hline AIF & 1.658 & 1.654 & $\mathrm{ClK}$ & 2.671 & 2.667 & IIn & 2.756 & 2.754 \\
\hline AlI & 2.540 & 2.537 & ClLi & 2.027 & 2.021 & IK & 3.051 & 3.048 \\
\hline $\mathrm{BF}$ & 1.267 & 1.263 & CINa & 2.365 & 2.361 & ILi & 2.398 & 2.392 \\
\hline $\mathrm{BH}$ & 1.247 & $1.236^{*}$ & $\mathrm{ClO}$ & 1.573 & 1.569 & INa & 2.715 & 2.711 \\
\hline $\mathrm{BN}$ & 1986 & $1981 *$ & CIRb & 9700 & 2. 787 & $\mathrm{IRb}$ & 3.180 & 3.177 \\
\hline $\mathrm{BO}$ & 1.210 & $1.204^{*}$ & CITI & 2.488 & 2.485 & ITl & 2.815 & 2.814 \\
\hline $\mathrm{B}_{2}$ & 1.594 & $1.590^{*}$ & $\mathrm{Cl}_{2}$ & 1.991 & $1.988^{*}$ & $\mathrm{I}_{2}$ & 2.669 & $2.667^{*}$ \\
\hline $\mathrm{BaO}$ & 1.942 & 1.940 & $\mathrm{CsF}$ & 2.347 & 2.345 & $\mathrm{Li}_{2}$ & 2.680 & $2.673^{*}$ \\
\hline $\mathrm{BrCl}$ & 2.139 & 2.136 & $\mathrm{CsI}$ & 3.318 & 3.315 & No & 1.154 & 1.151 \\
\hline $\mathrm{BrCs}$ & 3.075 & 3.072 & $\mathrm{CuF}$ & 1.749 & 1.745 & $\mathrm{NP}$ & 1.494 & I. .491 \\
\hline $\mathrm{BrF}$ & 1.759 & 1.756 & $\mathrm{FGa}$ & 1.778 & 1.774 & NS & 1.497 & 1.494 \\
\hline $\mathrm{BrGa}$ & 2.355 & 2.352 & FH & 0.926 & 0.917 & $N_{2}$ & 1.100 & $1.098^{*}$ \\
\hline $\mathrm{BrH}$ & 1.424 & 1.415 & $\mathrm{FI}$ & 1.913 & 1.910 & $\mathrm{OP}$ & 1.476 & $1.474^{*}$ \\
\hline $\mathrm{BrI}$ & 2.489 & 2.485 & FIn & 1.989 & 1.985 & $\mathrm{OPb}$ & 1.925 & 1.922 \\
\hline Brln & 2.545 & 2.543 & FK & 2.176 & 2.171 & OS & 1.484 & 1.481 \\
\hline $\mathrm{BrK}$ & 2.824 & 2.821 & FLi & 1.570 & 1.564 & $\mathrm{OSi}$ & 1.512 & 1.510 \\
\hline $\mathrm{BrLi}$ & 2.176 & 2.170 & $\mathrm{FN}$ & 1.321 & $1.317^{*}$ & OSn & 1.835 & 1.833 \\
\hline $\mathrm{BriNa}$ & 2.506 & 2.502 & $\mathrm{FNa}$ & 1.931 & 1.926 & $U_{2}$ & 1.211 & 1.208 \\
\hline $\mathrm{BrO}$ & 1.721 & 1.717 & FRb & 2.274 & 2.270 & $\mathrm{P}_{2}$ & 1.896 & $1.894 *$ \\
\hline $\mathrm{BrRb}$ & 2.948 & 2.945 & FS & 1.601 & & PbS & 2.289 & 2.287 \\
\hline $\mathrm{TlBr}$ & 2.620 & 2.618 & FTI & 2.088 & 2.084 & PbSe & 2.404 & 2.402 \\
\hline$C B r$ & & $1.621^{4}$ & $\mathrm{~F}_{2}$ & $1.417^{*}$ & & PbTe & 2.596 & 2.595 \\
\hline $\mathrm{CCl}$ & 1.649 & $1.645^{*}$ & $\mathrm{GaI}$ & 2.577 & 2.575 & $\mathrm{SSi}$ & 1.932 & 1.929 \\
\hline CF & 1.276 & $1.272^{*}$ & $\mathrm{GeO}$ & 1.627 & 1.625 & SSn & 2.211 & 2.209 \\
\hline $\mathrm{CH}$ & 1.131 & $1.120^{*}$ & $\mathrm{GeS}$ & 2.014 & 2.012 & $\mathrm{~S}_{2}$ & 1.892 & $1.889^{*}$ \\
\hline $\mathrm{CN}$ & 1.175 & 1. $172^{*}$ & $\mathrm{GeSe}$ & 2.136 & 2.135 & SeSi & 2.060 & 2.058 \\
\hline $\mathrm{CO}$ & 1.131 & 1.128 & GeTe & 2.342 & 2.340 & SeSn & 2.327 & 2.326 \\
\hline $\mathrm{CS}$ & 1.538 & 1.535 & H1 & 1.620 & 1.609 & $\mathrm{Si}_{2}$ & 2.249 & $2.246^{*}$ \\
\hline CSe & 1.679 & 1.676 & $\mathrm{HLi}$ & 1.604 & $1.595^{b}$ & $\mathrm{SnTe}$ & 2.524 & 2.523 \\
\hline \multirow[t]{2}{*}{$\mathrm{C}_{n}$} & 1.24 .6 & 1. $243^{*}$ & & & & & & 2.020 \\
\hline & & & & & & & & \\
\hline
\end{tabular}

a Distances are expected to have an accuracy of $0.001 \AA$ or better. Values indicated by an asterisk have been obtained from Rosen [9], the remaining values from Lovas and Tiemann [3].

${ }^{b}$ In this case the values are for the deuterated species, ${ }^{2} \mathrm{HLi}$. 


\section{Siructural Data Tables}

\begin{tabular}{|c|c|c|c|}
\hline & $\begin{array}{l}\text { Inorgo } \\
\text { Alumi }\end{array}$ & $\begin{array}{l}\text { olecul } \\
\text { hydrio }\end{array}$ & \\
\hline $\mathrm{AlH}_{2}$ & & & $\mathrm{C}_{2}$ \\
\hline Bond & Effertive & Angle & Effective \\
\hline AIH & $3.59 \mathrm{E}$ & HAlH & $119 \mathrm{E}$ \\
\hline
\end{tabular}

[1] G. Herzberg, Electronic Spectra of Polyatomic Molecules, D. Van Nostrand Co. Inc., Princeton, N.J., U.S.A., Table 62, 1960.

\section{Argon-Hydrogen chloride (I/I)}

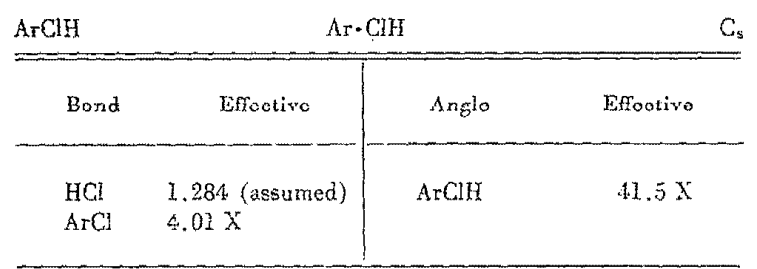

[1] S. E. Norich, D. Davies, S. Harris and W. Klemperer, J. Chem. Phys. 59, 2273 (2973).

Hydrogen Argon (1/1)

\begin{tabular}{lcc} 
Arfig & Ar. $\mathrm{H}_{2}$ & Undefined \\
\hline Bond & Efiective \\
\hline $\begin{array}{l}\text { Mean distance between center of mass of } \\
H_{2} \text { and Ar atom. }\end{array}$ & $3.94 \mathrm{X}$ \\
\hline
\end{tabular}

[1] A. R. W. McKellar and H. L. Welsh, J. Chem. Phys. 55, 595 (1971).

\section{Arsenic fribromide}

\begin{tabular}{|c|c|c|c|c|c|}
\hline Bond & Efective & Average & Angle & Effective & Average \\
\hline $\mathrm{AsBr}$ & $2.323 B$ & $2.324 \mathrm{~B}$ & BrAsBr & $99.8 \mathrm{~B}$ & $99.7 \mathrm{~B}$ \\
\hline
\end{tabular}

LIj A. G. Robiette, J. Mol. Struct. 35, 81 (1976).
Arsenk Trifuorlde (Trifluoroarsine)

\begin{tabular}{lc|cc}
\hline Bond & Effective & Aingle & Effective \\
\hline AsF & $1.712 X$ & FAsF & $102 X$ \\
\hline $\begin{array}{l}\text { Only one isotopic species studied. } \\
\text { [1] P. Kosliuk and S. Geschwind, J. Chem. Phys. 21, } 828 \text { (1953). }\end{array}$
\end{tabular}

\section{Arsino}

\begin{tabular}{cc|cc}
$\mathrm{AsH}_{2}$ & & $\mathrm{C}_{2 \mathrm{v}}$ \\
\hline Bond & Effective & Angle & Effective \\
\hline $\mathrm{AsH}$ & $1.518 \mathrm{C}$ & HAsH & $90.7 \mathrm{~B}$ \\
\hline
\end{tabular}

Ground electronic state is ${ }^{2} B_{1}$.

[1] R. N. Dixon, G. Duxbury and H. M. Lambertori, Proc. Roy. Soc. (London) A305, 271 (1968).

\section{Arsine}

$\mathrm{AsH}_{3}$

\begin{tabular}{lll|lll}
\hline Bond Effective Equilibrium & Angle & Efective & Equilibrium \\
\hline AsH & $1.520 \mathrm{~A}$ & $1.511 \mathrm{~A}$ & $\mathrm{HAsH}$ & $92.0 \mathrm{~A}$ & $92.1 \mathrm{~A}$ \\
\hline
\end{tabular}

[1] W. B. Olson, A. G. Maki and R. L. Sams, J. Mol. Spectrosc. $55,252(1975)$.

[2J F. Y. Chu and T. Oka, J. Chem. Phys. 60, 4612 (1974).

[3] K. Sarkia, D. Papousek and K. N. Rao, J. Mol. Spectrose. 37, 1 (1971).

[4j P. Helminger, E. L. Beeson and W. Gordy, Phys. Rev. A3, $122(1971)$.

\section{Boron chloride difluoride}

\begin{tabular}{ccc|cc} 
BCIF & $\mathrm{ClBF}_{2}$ & $\mathrm{C}_{20}$ \\
\hline Bond & Substitution & Effective & Angle & Efrective \\
\hline $\begin{array}{c}\mathrm{BCl} \\
\mathrm{BF}\end{array}$ & $1.728 \mathrm{C}$ & $3.315 \mathrm{C}$ & FBF & $118.1 \mathrm{C}$ \\
\hline
\end{tabular}

[1J H. W. Kroto and M. Maier, J. Mol. Spectrosc. 65, 280 (1977). 
Difluoroborane

\begin{tabular}{lcc|cc}
$\mathrm{BF}_{2} \mathrm{H}$ & & $\mathrm{C}_{2 v}$ \\
\hline \hline Bond & Substitution & Effective & Angle & Effective \\
\hline $\begin{array}{llll}\mathrm{BH} \\
\mathrm{BF}\end{array}$ & $1.189 \mathrm{C}$ & & $\mathrm{FBF}$ & $118.3 \mathrm{C}$
\end{tabular}

[1] T. Kasuya, W. J. Lafferty and D. R. Lide, J. Chem. Phys. 48, 1 (1968).

Difluorohydroxyborane

\begin{tabular}{ccc|cc}
$\mathrm{BF}_{2} \mathrm{HO}$ & \multicolumn{2}{c}{$\mathrm{F}_{2} \mathrm{BOH}$} & \multicolumn{2}{c}{$\mathrm{C}_{8}$} \\
\hline Bond & Substitution & Effective & Angle & Effective \\
\cline { 1 - 1 } & & & & \\
$\mathrm{OH}$ & $0.94 \mathrm{C}$ & $1.34 \mathrm{D}$ & $\mathrm{BOH}$ & $114 \mathrm{C}$ \\
$\mathrm{BO}$ & & $1.31 \mathrm{D}$ & $\mathrm{FBF}$ & $123 \mathrm{C}$ \\
$\mathrm{BF}$ & & $118 \mathrm{C}$ \\
\hline
\end{tabular}

[1] If. Takeo and R. F. Curl, J. Chem. Phys. 56, 4314. (1972).

1,1-Difluoroboranamine (Aminodifluoroborane)

\begin{tabular}{|c|c|c|c|c|c|}
\hline $\mathrm{BF}_{3} \mathrm{H}_{5} \mathrm{~N}$ & \multicolumn{4}{|c|}{$\mathrm{BF}_{\mathrm{N}} \mathrm{NH}_{2}$} & $\mathrm{C}_{2}$ \\
\hline Bond & Substitution & Effective & Angle & Substitution & Effective \\
\hline $\mathrm{BF}$ & & $1.325 \mathrm{C}$ & FBF & & $117.9 \mathrm{~B}$ \\
\hline $\mathrm{BN}$ & & $1.4 .02 \mathrm{D}$ & NHN & $116.9 \mathrm{~B}$ & \\
\hline $\mathrm{NH}$ & $1.003 \mathrm{~B}$ & & & & \\
\hline
\end{tabular}

[1] F. J. Lovas and D. R. Johnson, J. Chem. Phys. 59, 2347 (1973).

\section{Difluorophosphine Borane}

\begin{tabular}{lll|llr} 
& & & \\
\hline
\end{tabular}

Subscripts $s$ and a refer to in and out of the plane of symmetry, respectively.

[1] J. P. Pasinski and R. L. Kuczkowski, J. Chem. Phys., 54, 1903 (1971).
Boron Fluoride Oxide (Difluoroboroxy)

\begin{tabular}{cc|cc}
$\mathrm{BF}_{2} \mathrm{O}$ & \multicolumn{2}{c}{$\mathrm{F}_{2} \mathrm{BO}$} & $\mathrm{C}_{2 *}$ \\
\hline Bond & Effective & Angle & Effective \\
\hline $\mathrm{BF}$ & $\begin{array}{l}1.30 \mathrm{E} \\
\mathrm{BO}\end{array}$ & $\mathrm{FBF}$ & $126 \mathrm{E}$ \\
\hline
\end{tabular}

Gruund electionic state is ${ }^{\circ} \mathrm{B}$.

[1] C. W. Mathews, J. Mol. Spectrosc. 19, 203 (1966).

Boron Trifluoride (Trifluoroborane)

\begin{tabular}{lcc}
$\mathrm{BF}_{\mathrm{s}}$ & & $\mathrm{D}_{3 \mathrm{~h}}$ \\
\hline Bond & Effective & Equilibrium \\
\hline $\mathrm{BF}$ & $1.310 \mathrm{~B}$ & $1.307 \mathrm{~B}$
\end{tabular}

[l] S. G. W. Ginn, J. K. Kenny and J. Overend, J. Chem. Phys. $48,1571(1968)$.

[2] C. W. Brown and J. Overend, Can. J. Phys. 46, 977 (1968).

\section{Phosphine-trifluorobarane}

\begin{tabular}{cc|cc}
$\mathrm{BF}_{3} \mathrm{H}_{3} \mathrm{P}$ & $\mathrm{F}_{3} \mathrm{~B}-\mathrm{PH}_{3}$ & $\mathrm{C}_{3 \mathrm{w}}$ \\
\hline Bond & Effective & Angle & Efrective \\
\hline $\mathrm{PB}$ & $1.92 \mathrm{X}$ & $\mathrm{FBP}$ & $107 . \mathrm{X}$ \\
$\mathrm{BF}$ & $1.37 \mathrm{X}$ & $\mathrm{FBF}$ & $112 . \mathrm{X}$ \\
\hline
\end{tabular}

PH distance assumed to be $1.40 \AA$ and $\mathrm{xH} B$ angle assumed to be $117^{\circ}$.

[1] J. D. Odom, V. F. Kalasinsky, and J. R. Durig, Inorg. Chem. 14, 2837 (1975).

\section{Phosphorus Trifluoride-Borane}

\begin{tabular}{lll|lll}
$\mathrm{BF}_{3} \mathrm{H}_{3} \mathrm{P}$ & \multicolumn{2}{c}{$\mathrm{F}_{3} \mathrm{PBH}_{3}$} & $\mathrm{C}_{3 \mathrm{v}}$ \\
\hline Bond & Substitution & Effective & Angle & Substitution & Effective \\
\hline $\mathrm{BH}$ & $1.207 \mathrm{~B}$ & & $\mathrm{HBH}$ & $115.1 \mathrm{C}$ & \\
$\mathrm{PF}$ & & $1.538 \mathrm{D}$ & $\mathrm{FPF}$ & & $99.8 \mathrm{D}$ \\
$\mathrm{PB}$ & & $1.836 \mathrm{D}$ & & & \\
\hline
\end{tabular}

[1] R. L. Kuczkowski and D. R. Lide, Jr., J. Chem. Phys. 46, 35\% (1967). 
Thioxoborane(3) (Thioborane)

\begin{tabular}{cc} 
BHS & SBS \\
Bond & Substitution \\
BH & $1.169 \mathrm{~A}$ \\
BS & $1.599 \mathrm{~A}$ \\
\hline
\end{tabular}

[1] E. F. Pearson and R. U. McCormick, J. Chem. Phys. 58, 1619 (1973).

Borane(2)

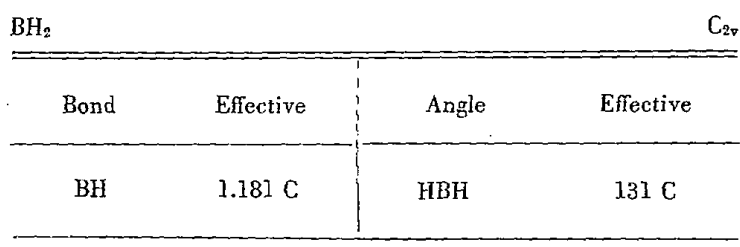

Ground state is ${ }^{2} \mathrm{~B}_{1}$.

[1] G. Herzberg and J. W. C. Johns, Proc. Roy. Soc. (London) A298, 142 (1967).

Phosphine-Borane

\begin{tabular}{llll}
$\mathrm{BH}_{6} \mathrm{P}$ & & $\mathrm{H}_{3} \mathrm{BPH}_{3}$ & $\mathrm{C}_{3 \mathrm{v}}$ \\
\hline Bond & Substitution & Angle & Substitution \\
& & & \\
$\mathrm{BP}$ & $1.937 \mathrm{~B}$ & $\mathrm{PBH}$ & $103.6 \mathrm{~B}$ \\
$\mathrm{BH}$ & $1.212 \mathrm{~B}$ & $\mathrm{BPH}$ & $116.9 \mathrm{~B}$ \\
$\mathrm{PH}$ & $1.399 \mathrm{~B}$ & $\mathrm{HBH}$ & $114.6 \mathrm{~B}$ \\
& & $\mathrm{HPJH}$ & $101.3 \mathrm{~B}$
\end{tabular}

[1] J. R. Durig, Y. S. Li, L. A. Carreira, and J. D. Odon, J. Amer. Chem. Soc. 95, 2491 (1973).

\section{Boron Dioxide}

Bond
BO

Ground electronic state is $2 \Pi_{\mathrm{g}}$.

[1] I. W. C. Johns, Can. J. Phys. 39, 1738 (1961).
Bramodiborane(6)

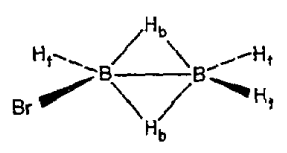

$\mathrm{B}_{2} \mathrm{BrH}_{5}$

\begin{tabular}{|c|c|c|c|c|c|}
\hline Bond & Substitution & Effective & Angle & Substitution & Effective \\
\hline$B B$ & $1.773 \mathrm{~B}$ & & $\mathrm{BBBr}$ & $121.4 \mathrm{C}$ & \\
\hline $\mathrm{BBr}$ & $1.930 \mathrm{~B}$ & & $\mathrm{BBH}_{\mathrm{t}}$ & & $119.9 \times$ \\
\hline $\mathrm{H}_{3} \mathrm{H}_{\mathrm{b}}$ & & I. $954 \mathrm{X}$ & $\mathrm{H}_{\mathrm{b}} \mathrm{BH}_{\mathrm{b}}$ & & $95.6 \mathrm{X}$ \\
\hline $\mathrm{BH}_{t}$ & & $1.196 \mathrm{X}$ & $\mathrm{BH}_{\mathrm{b}} \mathrm{B}$ & & $84.4 \mathrm{X}$ \\
\hline
\end{tabular}

[1] A. r. Ferguson and C. D. Cornwell, I. Chem. Phys. 53, 185$]$ (1970).

\section{1,2,4,3,5-Trioxadiboralane}

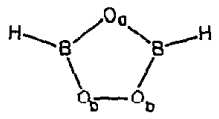

\begin{tabular}{llll} 
Bond & Substitution & Angle & Substitution \\
\hline & & & \\
$\mathrm{BH}$ & $1.182 \mathrm{~B}$ & $\mathrm{COB}$ & $104.0 \mathrm{C}$ \\
$\mathrm{BO}_{\mathrm{a}}$ & $1.380 \mathrm{~B}$ & $\mathrm{OBO}$ & $113.0 \mathrm{C}$ \\
$\mathrm{BO}_{\mathrm{b}}$ & $1.365 \mathrm{~B}$ & $\mathrm{BOO}$ & $105.0 \mathrm{C}$ \\
$\mathrm{O}_{\mathrm{b}} \mathrm{O}_{\mathrm{b}}$ & $1.470 \mathrm{~A}$ & $\mathrm{HBO}$ & $126.3 \mathrm{C}$
\end{tabular}

[1] W. V. F. Brooks, C. C. Costain and R. F. Porter, J. Chem. Phys. 47, 4186 (1967).

\section{Diborane(6)}

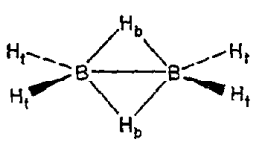

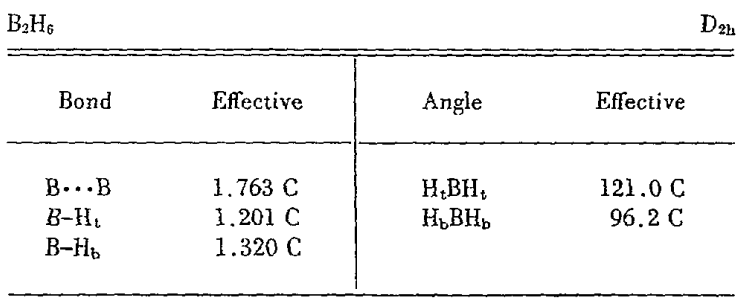

[1] W. J. Lafferty, A. G. Maki and T. D. Coyle, J. Mol. Spectrosc. 33, 345 (1970). 


\section{A minodiborane}

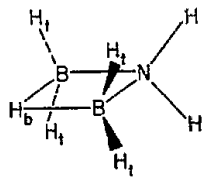

$\mathrm{B}_{2} \mathrm{H}_{7} \mathrm{~N}$

\begin{tabular}{lll|lrl}
\hline Bond & Substitution Effective & Angle & Substitution & Effective \\
\hline & & & & & \\
$\mathrm{BB}$ & $1.916 \mathrm{~B}$ & & $\mathrm{BNB}$ & $75.9 \mathrm{~A}$ & \\
$\mathrm{BN}$ & $1.558 \mathrm{~B}$ & & $\mathrm{BH}_{3} \mathrm{~B}$ & $90.0 \mathrm{~B}$ & \\
$\mathrm{BH}_{\mathrm{b}}$ & $1.355 \mathrm{C}$ & & $\mathrm{H}_{\mathrm{t}} \mathrm{BH}_{\mathrm{t}}$ & $121.0 \mathrm{~B}$ & \\
$\mathrm{BH}_{\mathrm{t}}$ & $1.193 \mathrm{~B}$ & & $\mathrm{NBH}_{\mathrm{t}}$ & $113.7 \mathrm{~B}$ & \\
$\mathrm{NH}$ & & $1.005 \mathrm{C}$ & $\mathrm{HNH}$ & & $111.0 \mathrm{D}$ \\
& & & & & \\
\hline
\end{tabular}

[1] K. K. Lau, A. B. Burg, and R. A. Beaudet, Inorg. Chem. 13, $2787(1974)$

Penfaborane (9)

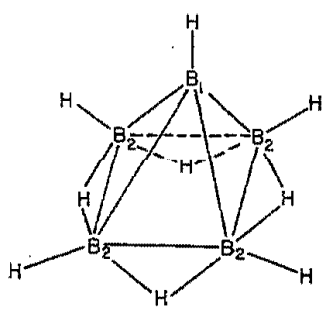

$\mathrm{B}_{6} \mathrm{H}_{9}$

$\mathrm{C}_{\$ \mathrm{~V}}$

\begin{tabular}{cc} 
Bond & Effective \\
\hline$B_{1} \mathrm{~B}_{2}$ & $1.687 \mathrm{C}$ \\
$\mathrm{B}_{2} \mathrm{~B}_{2}$ & $1.800 \mathrm{C}$
\end{tabular}

Hydrogen atoms are not uniquely determined, but data are consistent with five single $\mathrm{BH}$ bonds and four bridging hydrogens as shown.

[I] H. J. Hrostowski and R. J. Myers, J. Chem. Phys. 22, 262 (1954).

[2] H. J. Hrostowski, R. J. Myers and G. C. Pimentel, J. Chem. Phys. 20, 518 (1952).
Sulfuryl bromide fluoride

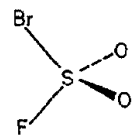

$\mathrm{BrFO}_{2} \mathrm{~S}$

\begin{tabular}{cc|cc}
\hline Bond & Effective & Angle & Effective \\
\hline $\mathrm{SBr}$ & $2.155 \mathrm{X}$ & $\mathrm{FSBr}$ & $100.6 \mathrm{X}$ \\
\hline
\end{tabular}

SO, SF, and OSO were assumed to be $1.407,1.560$, and 123.\%, respectively.

[1] J. M. Raley and J. E. Wollrab, J. Mol. Spectrosc. 48, 100 (1973).

Bromine fluoride

(Bromine trifluoride)

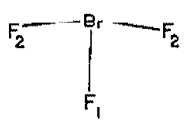

$\mathrm{BrF}_{3}$

\begin{tabular}{cc|cc}
\hline Bond & Effective & Angle & Effective \\
\hline $\mathrm{BrF}_{1}$ & $1.721 \mathrm{C}$ & $\mathrm{F}_{1} \mathrm{BrF}_{2}$ & $86.2 \mathrm{C}$ \\
$\mathrm{BrF}_{2}$ & $1.810 \mathrm{~B}$ & & \\
\hline
\end{tabular}

[1] D. W. Magnuson, J. Chem. Phys. 27, 223 (1957).

\section{Bromotrifluorosilane}

\begin{tabular}{cc|cc}
$\mathrm{BrF}_{2} \mathrm{Si}$ & \multicolumn{2}{c}{$\mathrm{SiF}_{3} \mathrm{Br}$} & $\mathrm{C}_{3 \mathrm{v}}$ \\
\hline \hline Bond & Effective & Angle & Enecitive \\
\hline SiF & $1.560 \mathrm{~B}$ & FSiF & $108.5 \mathrm{D}$ \\
$\mathrm{SiBr}$ & $2.153 \mathrm{D}$ & &
\end{tabular}

Bond distances determined by assuming the value for FSiF. [1] J. Sheridan and W. Gordy, J. Chem. Phys. 19, 965 (1951).

Sulfur bromide fluoride

(Sulfur pentafluoride bromide)

\begin{tabular}{ccc}
$\mathrm{BrF}_{5} \mathrm{~S}$ & $\mathrm{BrSF}_{5}$ & $\mathrm{C}_{40}$ \\
\hline \hline Bond & Effective \\
\hline $\mathrm{SBr}$ & $2.190 \mathrm{X}$ \\
$\mathrm{SF}$ & $1.597 \mathrm{X}$
\end{tabular}

Angle $\mathrm{F}$ (eq) $\mathrm{SF}(\mathrm{ax})$ was assumed to be $88.0^{\circ}$, and all $\mathrm{SF}$ bond lengths were assumed to be equal.

[I] E. W. Neuvar and A. W. Jache, J. Chem. Phys. 39, 596 (1963). 
Bromogermane

\begin{tabular}{lcc|ll}
$\mathrm{BrGeH}_{3}$ & $\mathrm{BrGeH}_{3}$ & $\mathrm{C}_{3 \mathrm{v}}$ \\
\hline Bond & Effective & Substitution & Angle & Effective \\
\hline $\begin{array}{l}\mathrm{Ge}-\mathrm{H} \\
\mathrm{Ge}-\mathrm{Br}\end{array}$ & $1.535 \mathrm{D}$ & & \\
\hline
\end{tabular}

The substitution distance derived in Ref. I was combined with the $A_{0}$ value obtained in Ref. 2 by use of the zeta sum rule to cal. culate the molecular parameters.

[1] S. N. Wolf and L. C. Krisher, I. Chem. Phys. 56, 1040 (1972).

[2] K. H. Rhee and M. K. Wilson, J. Chem. Phys. 43, 333 (1965).

\begin{tabular}{ll|ll}
\multicolumn{2}{c}{ Bromosilylene } \\
BrHSi & Angle & Effective \\
\hline $\mathrm{SiBr}$ & $\mathrm{HSiBr}$ & $\mathrm{C}$ \\
\hline
\end{tabular}

SiH distance assumed (1.56I $\hat{A})$.

[1] G. Herzberg and R. D. Verma, Can. J. Phys. 42, 395 (1964).

\begin{tabular}{|c|c|c|c|}
\hline \multicolumn{4}{|c|}{ Bromosilene } \\
\hline $\mathrm{BrH}_{3} \mathrm{Si}$ & \multicolumn{2}{|c|}{$\mathrm{BrSiH}$} & $\mathrm{C}_{3 \mathrm{v}}$ \\
\hline Bond & Substitution & Angle & Substitution \\
\hline $\mathrm{SiBr}$ & $2.210 \mathrm{~B}$ & $\mathrm{HSiB}_{\mathrm{I}}$ & $107.9 \mathrm{~B}$ \\
\hline $\mathrm{SiH}$ & $1.481 \mathrm{~B}$ & & \\
\hline
\end{tabular}

[1] R. Kawley, P. M. McKinney, and A. G. Robiette, J. Mol. Spectrosc. 34, 390 (1970).

\section{Bromostannane}

\begin{tabular}{|c|c|c|c|c|}
\hline $\mathrm{BrH}_{3} \mathrm{Sn}_{\mathrm{n}}$ & & $\mathrm{Br} \mathrm{SnH}_{n}$ & & $C_{v v}$ \\
\hline Bond & Substitution & Effective & Angle & Effective \\
\hline $\begin{array}{l}\mathrm{SnBr} \\
\mathrm{SnH}\end{array}$ & $2.469 A$ & $1.76 \mathrm{X}$ & $\mathrm{HSn} B r$ & $106 \mathrm{C}$ \\
\hline
\end{tabular}

[1] S. N. Wolf, L. C. Krisher, and R. A. Gsell, J. Chem. Phys. 54, 4605 (1971).

\section{Nitrosyl Bromide}

BrNO

\begin{tabular}{|c|c|c|c|}
\hline Bond & Effective & Angle & Effective \\
\hline
\end{tabular}

$\mathrm{NBr} \quad 2.140 \mathrm{C}$

BrNO

$114.5 \mathrm{C}$

[1] D. J. Millen and D. Mitra, Trans. Faraday Soc. 65, 1975 (1969).

Fluorotribromosilane

\begin{tabular}{ll|ll}
$\mathrm{Br}_{3} \mathrm{FSi}$ & Effective & Angle & Effective \\
\hline $\mathrm{SiBr}$ & $2.171 \mathrm{X}$ & $\mathrm{BrSiBr}$ & $111.6 \mathrm{X}$
\end{tabular}

$\mathrm{SiF}=1.560$ was assumed.

[1] M. Mitzlaff, R. Holm and H. Hartmann, Z. Naturforsch. 23a, 1819 (1968).

\section{Tribromosilane}

\begin{tabular}{ll|ll} 
Bond & Effective & Angle & Effective \\
\hline & & & \\
$\mathrm{SiH}$ & $1.494 \mathrm{C}$ & $\mathrm{BrSiBr}$ & \\
$\mathrm{SiBr}$ & $2.170 \mathrm{E}$ & & \\
\hline
\end{tabular}

[1] M. Mitzlaff, R. Holm, and H. Hartmann. Z. Naturforsch. 23a. 65 (1968).

\section{Chloryl fluoride}

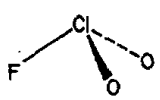

$\mathrm{ClFO}_{2}$

\begin{tabular}{|c|c|c|c|}
\hline Bond & Substitution & Angle & Substitution \\
\hline $\mathrm{ClF}$ & $1.696 \mathrm{~B}$ & $\mathrm{OClO}$ & $115.2 \mathrm{~A}$ \\
\hline $\mathrm{ClO}$ & $1.118 \mathrm{~B}$ & $\mathrm{FClO}$ & $101.7 \mathrm{~A}$ \\
\hline
\end{tabular}

[1] C. R. Parent and M. C. L. Gerry, J. Mol. Spectrosc. 49, 343 (1974). 
Sulfuryl chloride fluoride

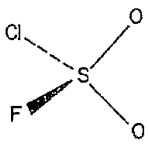

\begin{tabular}{|c|c|c|c|}
\hline Bond & Effective & Angle & Effective \\
\hline so & $1.108^{8}$ & oso & $123.7 \mathrm{X}$ \\
\hline$S F$ & $1.550 \mathrm{X}$ & $\mathrm{FSCl}$ & $99.0^{\mathrm{s}}$ \\
\hline SCl & $1.985 X$ & $\begin{array}{l}\text { OSF } \\
\text { OSCl }\end{array}$ & $\begin{array}{l}107.5 \times \\
107.5 X\end{array}$ \\
\hline
\end{tabular}

a Assumed values.

[1] C. S. Folt and M. C. L. Gerry, Chem. Phys. Lett. 9, 621 (1971).

\section{Phosphorous chlorĩde difluoride}

(Chlorodifluorophosphine)

\begin{tabular}{cc|cc}
$\mathrm{CF}_{2} \mathrm{P}$ & $\mathrm{PFCl}$ & $\mathrm{C}$ \\
\hline Bond & Effective & Angle & Effective \\
\hline $\mathrm{PF}$ & $1.571 \mathrm{~B}$ & FPF & $97.3 \mathrm{~B}$ \\
$\mathrm{PCl}$ & $2.030 \mathrm{C}$ & $\mathrm{FPCl}$ & $99.2 \mathrm{C}$ \\
\hline
\end{tabular}

[1] A. H. Brittain, J. E. Smith and R. H. Schwendeman, Inorg. Chem. 11, 39 (1972).

\section{Chlorine Fluoride}

\begin{tabular}{llll} 
& & & \\
\hline Bond & Effective & Angle & Effective \\
\hline $\begin{array}{ll}\mathrm{ClF}_{3} \\
\mathrm{ClF}_{2}\end{array}$ & $1.598 \mathrm{~B}$ & $\mathrm{~F}_{1} \mathrm{ClF}_{2}$ & $87.5 \mathrm{~B}$ \\
\hline
\end{tabular}

[1] D. F. Smith, J. Chem. Phys. 21, 609 (1953).
Chlorotrifluorogermane

\begin{tabular}{cccc}
$\mathrm{CIF}_{3} \mathrm{Ge}$ & \multicolumn{2}{c}{$\mathrm{GeF}_{3} \mathrm{Cl}$} & $\mathrm{C}_{3 \mathrm{v}}$ \\
\hline \hline Bond & Effective & Angle & Effective \\
\hline $\mathrm{GeF}$ & $\begin{array}{l}1.688 \mathrm{D} \\
\mathrm{GeCl}\end{array}$ & FGeF & $107.5 \mathrm{E}$ \\
\hline
\end{tabular}

[1] W. E. Anderson, J. Sheriaan and W. Gordy, Phys. Rev. 81, $819(1951)$.

\section{Chlorotrifiuorosilane}

\begin{tabular}{|c|c|c|c|}
\hline $\mathrm{ClF}_{3} \mathrm{Si}$ & \multicolumn{2}{|c|}{$\mathrm{SiF}_{3} \mathrm{Cl}$} & $C_{5 p}$ \\
\hline \multicolumn{2}{|r|}{ Bond } & \multicolumn{2}{|c|}{ Effective } \\
\hline \multicolumn{2}{|r|}{$\underset{\mathrm{SiF}}{\mathrm{SiCl}}$} & \multicolumn{2}{|c|}{$1.560 \mathrm{C}$} \\
\hline \multicolumn{4}{|c|}{$\begin{array}{l}\text { Bond distances obtained by assuming FSiF }=108.5 \pm 2^{\circ} \text {. } \\
\text { [1] J. Sheridan and W. Gordy, J. Chem. Phys. 19, } 965 \text { (1951). }\end{array}$} \\
\hline $\mathrm{ClF}_{5}$ & & & $\mathrm{C}_{4}$ \\
\hline Bond & Effective & Angle & Effective \\
\hline $\mathrm{CIF}$ & $1.65 \mathrm{X}$ & $\begin{array}{l}F_{a x} \mathrm{ClF}_{\mathrm{eq}} \\
\mathrm{F}_{\mathrm{ga}} \mathrm{ClF}_{4 \mathrm{a}}\end{array}$ & $\begin{array}{l}86.5 \mathrm{X} \\
89.9 \mathrm{X}\end{array}$ \\
\hline
\end{tabular}

The $\mathrm{ClF}_{\mathrm{as}}$ and $\mathrm{CIF}_{\text {tal }}$ distances have been assumed to be equal.

[1] H. K. Bodenseh, W. Hüttner, and P. Nowicki, Z. Naturforsch. $31 a, 1638$ (1976).

[2] P. Goulet, R. Jurek, and J. Chanussot, J. de Phys. 37, 495 (1976).

[3] R. Jurek, P. Suzeau, J. Chanussot, and J. P. Champion, J. de Phys. 35, 533 (1974).

\section{Tellurium pentafluoride chloride}

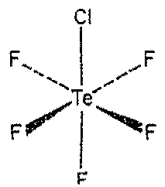

$\mathrm{CHF}_{5} \mathrm{Te}$

$\mathrm{Cu}_{4 \mathrm{v}}$

\begin{tabular}{lll|ll}
\hline \hline Bond Substitution Effective & Angle Substitution Effective \\
\hline $\mathrm{TeCl}$ & $2.250 \mathrm{~B}$ & & $\mathrm{~F}_{\mathrm{or} 2} \mathrm{TeF}_{\mathrm{ax}}$ & $88.3 \mathrm{X}$ \\
$\mathrm{TeF}$ & $1.831 \mathrm{X}$ & & \\
\hline
\end{tabular}

The $\mathrm{TeF}_{\mathrm{cq}}$ distance has been assumed equal to the $\mathrm{TeF}_{\mathrm{z}}$ distance. [1] A. C. Legon, J. Chem. Soc., Faraday Trans. 69, 29 (1973). 
Tungsten pentafluoride chloride

\begin{tabular}{|c|c|c|c|c|}
\hline $\mathrm{ClF}_{6} \mathrm{~W}$ & & $\mathrm{CJWF} \mathrm{F}_{5}$ & & $\mathrm{C}_{48}$ \\
\hline Bond & Substitution & Effective & Angle & Effective \\
\hline $\mathrm{WCl}$ & $2.252 \mathrm{~B}$ & \multirow[b]{2}{*}{$1.836 \mathrm{X}$} & $\theta^{\mathrm{b}}$ & $88.7 \mathrm{X}$ \\
\hline WFa & & & & \\
\hline
\end{tabular}

All WF bonds assumed equal.

b $F_{\text {axial }}$ WF equatoria! angle.

[1] A. C. Legon, Trans Faraday Soc. 65, 2595 (1969).

\section{Hypochlorous acid}

\begin{tabular}{|c|c|c|c|}
\hline $\mathrm{CIHO}$ & \multicolumn{2}{|c|}{$\mathrm{HOCl}$} & $\mathrm{C}_{\mathrm{s}}$ \\
\hline Bond & Substitution & Angle & Substitution \\
\hline $\mathrm{OH}$ & $0.067 \mathrm{C}$ & $\mathrm{HOCl}$ & $102.4 \mathrm{G}$ \\
\hline $\mathrm{OCI}$ & $1.690 \mathrm{~A}$ & & \\
\hline
\end{tabular}

[1] A. M. Mirri, F. Scappini and G. Cazzoli, J. Mol. Spectrosc. 38, 227 (1971).

[2] D. C. Lindsey, D. G. Lister and D. J. Millen, Chem. Comm. 1969, 950 (1969)

[3] R. A. Ashby, J. Mol. Spectrosc. 23, 439 (1967).

\section{Chlorosilylene}

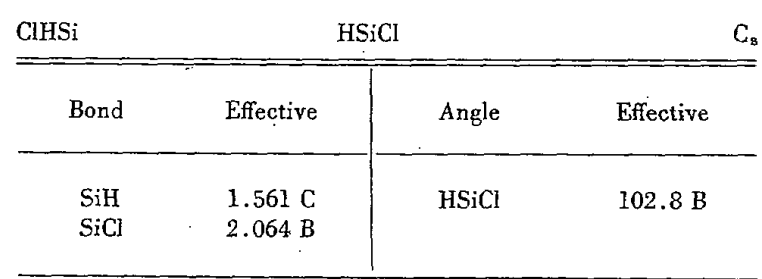

[1] G. Herzberg and R. D. Verma, Can. J. Phys. 42, 395 (1964).

\begin{tabular}{|c|c|c|c|}
\hline \multicolumn{4}{|c|}{$\begin{array}{c}\text { Chloramide } \\
\text { (Monochloramine) }\end{array}$} \\
\hline $\mathrm{ClH}_{2} \mathrm{~N}$ & \multicolumn{2}{|c|}{$\mathrm{CINH}_{2}$} & $\mathrm{C}_{\mathrm{s}}$ \\
\hline Bond & Substitution & Angle & Substitution \\
\hline $\mathrm{NH}$ & $1.017 \mathrm{C}$ & $\mathrm{HNCl}$ & $103.7 \mathrm{~B}$ \\
\hline $\mathrm{NCl}$ & $1.748 \mathrm{~A}$ & HNH & $107.4 \mathrm{D}$ \\
\hline
\end{tabular}

[1] G. Cazzoli, D. G. Lister and P. G. Favero, J. Mol. Spectrosc. 42, 286 (1972)

[2] G. E. Moore and R. M. Badger, J. Amer. Chem. Soc. 74, 6076 (1952).

\section{Chlorosilane}

\begin{tabular}{|c|c|c|c|c|c|}
\hline $\mathrm{ClH}_{3} \mathrm{Si}$ & \multicolumn{4}{|c|}{$\mathrm{H}_{3} \mathrm{SiCl}$} & $\mathrm{C}_{3 \mathrm{v}}$ \\
\hline Bond & Substitution & Effective & Angle & Substitution & Effective \\
\hline $\mathrm{SiCl}$ & $2.048 \mathrm{~A}$ & $2.049 \mathrm{~A}$ & $\mathrm{HSiCl}$ & $107.9 \mathrm{~B}$ & $108.7 \mathrm{~B}$ \\
\hline $\mathrm{SiH}$ & $1.482 \mathrm{~B}$ & $1.485 \mathrm{~B}$ & & & \\
\hline
\end{tabular}

[1] R. Kewley, P. M. McKinney and A. G. Robiette, J. Mol. Spectrosc. 34, 390 (1970).

\section{Chlorostannane}

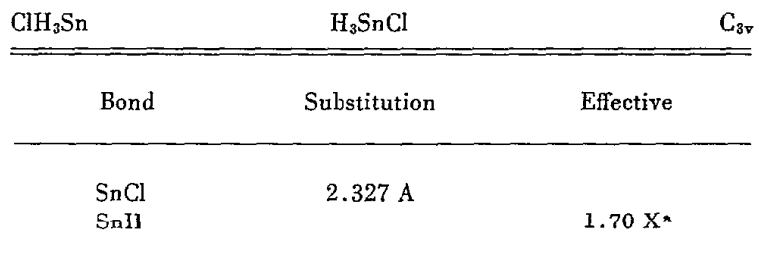

"Value obtained by assuming methyl group to be tetrahedral.

[1] L. C. Krisher, R. A. Gsell and J. M. Bellama, J. Chem. Phys. 54, 2287 (1971).

\section{Nitrosyl chloride}

ClNo $\quad \mathrm{O}=\mathrm{N}-\mathrm{Cl} \quad \mathrm{C}_{y_{3}}$

\begin{tabular}{cc|cc}
\hline Bond & Substitution & Angle & Substitution \\
\hline NO & $\begin{array}{l}1.139 \mathrm{C} \\
\mathrm{NCl}\end{array}$ & ONCl & $113.3 \mathrm{C}$ \\
\hline
\end{tabular}

\section{Nitryl chloride}

$\mathrm{ClNO}_{2}$

\begin{tabular}{cc|cc}
\hline Bond & Effotivo & Angle & Efeutive \\
\hline $\mathrm{NCl}$ & $1.840 \mathrm{~B}$ & ONO & $130.6 \mathrm{C}$ \\
$\mathrm{NO}$ & $1.202 \mathrm{~B}$ & & \\
\hline
\end{tabular}

[1] D. J. Millen and K. M. Sinnott, J. Chem. Soc. 1958, 350.

[2] L. Clayton, Q. Williams and T. L. Weatherly, J. Chem. Phys. 30, 1328 (1959), Errata, J. Cham. Phys., 31, 554 (1959).

[3] T. Oka and Y. Morino, J. Mol. Spectrosc. 11, 349 (1963). 


\section{Thiazyl chloride}

\begin{tabular}{lll|llll} 
CINS & \multicolumn{2}{c}{$\mathrm{N} \equiv \mathrm{S}-\mathrm{CI}$} & $\mathrm{C}_{8}$ \\
\hline Bond & Substitution & Effective & Angle & Substitution & Effective \\
& & & & & & \\
$\mathrm{NS}$ & $1.450 \mathrm{~A}$ & $1.452 \mathrm{~B}$ & $\mathrm{NSCl}$ & $117.7 \mathrm{~A}$ & $117.9 \mathrm{~B}$ \\
$\mathrm{SCl}$ & $2.161 \mathrm{~A}$ & $2.161 \mathrm{~B}$ & & &
\end{tabular}

The effective structure is an average of that determined in three independent ways.

1] T. Beppu, E. Hirota and Y. Morino, J. Mol. Spectrosc. 36, $386(1970)$.

Chlorine azide

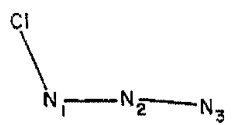

$\mathrm{ClN}_{3}$

\begin{tabular}{lc|cc}
\hline Bond & Substitution & Angle & Substitution \\
\hline $\mathrm{CN}_{3}$ & $1.745 \mathrm{C}$ & $\mathrm{CIN}_{\mathrm{I}} \mathrm{N}_{2}$ & $108.7 \mathrm{C}$ \\
$\mathrm{N}_{1} \mathrm{~N}_{2}$ & $1.252 \mathrm{C}$ & $\mathrm{N}_{3} \mathrm{~N}_{2} \mathrm{~N}_{3}$ & $171.9 \mathrm{C}$ \\
$\mathrm{N}_{2} \mathrm{~N}_{3}$ & $1.133 \mathrm{C}$ & & \\
\hline
\end{tabular}

[1] R. L. Cook and M. C. L. Gerry, J. Chem. Phys. 53, 2525 (1970).

\section{Chlorine dioxide}

\begin{tabular}{lll|lll}
$\mathrm{ClO}_{2}$ & \multicolumn{2}{c}{$0 \mathrm{ClO}$} & $\mathrm{C}$ \\
\hline Bond & Substitution & Average & Angle & Substitution & Average \\
\hline $\mathrm{ClO}$ & $1.471 \mathrm{~B}$ & $1.476 \mathrm{~B}$ & $0 \mathrm{ClO}$ & $117.6 \mathrm{~B}$ & $117.5 \mathrm{~B}$ \\
\hline
\end{tabular}

Ground state is ${ }^{2} B_{1}$.

[1] A. H. Clark, J. Mol. Struct. 7, 485 (1971).

[2] F. R. Curl, Jr., R. F. Heidelberg and J. L. Kinsey, Phys. Rev. 125, 1993 (1962)

[3] R. F. Curl, Jr., J. L. Kinsey, J. G. Baker, J. C. Baird, G. R. Bird, R. F. Heidelberg, T. M. Sugden, D. R. Jenkins and C N. Kenney, Phys. Rev. 121, 1119 (1961).

\section{Chlorotrioxorhenium}

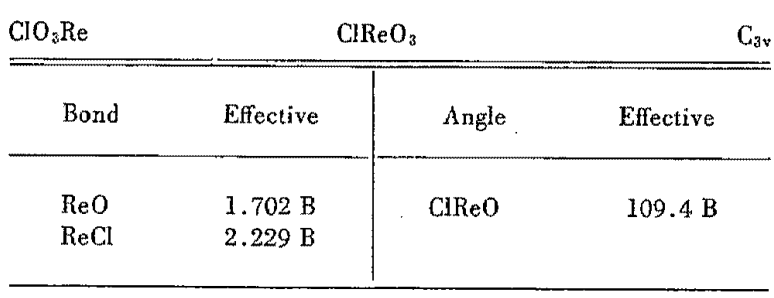

[1] E. Amble, S. L. Miller, A. L. Schawlow and C. H. Townes, J. Chem. Phys. 20, 192 (1952).

[2] J. F. Lotspeich, A. Javan and A. Engelbrecht, J. Chem. Phys. 31, 633 (1959).
Dichlorosilane

\begin{tabular}{cc|cc}
$\mathrm{Cl}_{2} \mathrm{H}_{2} \mathrm{Si}$ & & $\mathrm{C}_{2 \mathrm{v}}$ \\
\hline Bond & Substitution & Angle & Substitution \\
\hline $\mathrm{SiCl}$ & $2.033 \mathrm{~A}$ & $\mathrm{CISiCl}$ & $109.7 \mathrm{~B}$ \\
$\mathrm{SiH}$ & $1.480 \mathrm{D}$ & $\mathrm{HSiH}$ & $111.3 \mathrm{C}$
\end{tabular}

Hydrogen parameters are not strictly substitution parameters, but should be nearly so.

[1] R. W. Davis and M. C. L. Gerry, J. Mol. Spectrosc. 60, 117 (1976).

Dichlorine oxide

\begin{tabular}{|c|c|c|c|c|c|}
\hline $\mathrm{Cl}_{2} \mathrm{O}$ & \multicolumn{5}{|c|}{$\mathrm{ClOCl}$} \\
\hline Bond & Substitution & Effective & Angle & Substitution & Effective \\
\hline $\mathrm{ClO}$ & $1.700 \mathrm{~A}$ & $1.700 \mathrm{~A}$ & $\mathrm{ClOCl}$ & $110.9 \mathrm{~B}$ & $110.9 \mathrm{~B}$ \\
\hline
\end{tabular}

[1] G. E. Herberick, R. H. Jackson and D. J. Millen, J. Chem. Soc. 1966, 1156 (1966).

\section{Sulfur dichloride}

$\mathrm{Cl}_{2} \mathrm{~S}$

\begin{tabular}{cccc}
\hline \hline Bond & Substitution & Average & Effective \\
\hline ClS & $2.014 \mathrm{~A}$ & $2.015 \mathrm{~A}$ & $2.014 \mathrm{~A}$ \\
\hline \hline Angle & Substitution & Average & Effective \\
\hline CISCl & $102.6 \mathrm{~A}$ & $102.7 \mathrm{~A}$ & $102.7 \mathrm{~A}$
\end{tabular}

[1] R. W. Davis and M. C. L. Gerry, J. Mol. Spectrosc. 65, 455 (1977).

[2] J. L. Murray, W. A. Little, Q. Williams and T. L. Weatherby, J. Chem. Phys. 65, 985 (1976).

Trichlorofluorosilane

\begin{tabular}{cc|cc}
$\mathrm{Cl}_{n} \mathrm{FSi}$ & \multicolumn{2}{c}{$\mathrm{FSiCl}_{3}$} & \multicolumn{1}{c}{$\mathrm{C}_{*}$} \\
\hline \hline Bond & Effective & Angle & Effective \\
\hline & & & \\
$\mathrm{SiF}$ & $1.520 \mathrm{~B}$ & FSiCl & $109.5 \mathrm{~B}$ \\
$\mathrm{SiCl}$ & $2.019 \mathrm{~B}$ & $\mathrm{ClSiCl}$ & $109.4 \mathrm{~B}$ \\
\hline
\end{tabular}

[1] R. Holm, M. Mitzloff and H. Hartmann, Z. Naturforschg. 22a, 1287 (1967). 
Trichlorogermane

\begin{tabular}{cc|cc}
$\mathrm{Cl}_{3} \mathrm{GeH}$ & \multicolumn{1}{c}{$\mathrm{HGeCl}_{3}$} & $\mathrm{C}_{3 \mathrm{r}}$ \\
\hline Bond & Effective & Angle & Effective \\
\hline $\mathrm{GeH}$ & $1.55 \mathrm{E}$ & $\mathrm{CGeCl}$ & $2.114 \mathrm{~A}$ \\
$\mathrm{GeCl}$ & $2.114 \mathrm{~A}$ & & \\
\hline
\end{tabular}

[1] P. Venkateswarlu, R. C. Mockler, and W. Gordy, J. Chem. Phys. 21, 1713 (1953).

\section{Trichlorosilane}

\begin{tabular}{lll|lll}
$\mathrm{Cl}_{3} \mathrm{HSi}$ & \multicolumn{2}{c}{$\mathrm{HSiCl}_{3}$} & $\mathrm{C}_{3 \mathrm{~V}}$ \\
Bond & Substitution & Effective & Angle & Substitution & Effective \\
\hline $\mathrm{HSi}$ & $1.465 \mathrm{~A}$ & $1.47 \mathrm{X}$ & $\mathrm{HSiCl}$ & $108.3 \mathrm{~B}$ & $109.5 \mathrm{~B}$ \\
$\mathrm{CISi}$ & $2.019 \mathrm{~A}$ & $2.021 \mathrm{~A}$ & $\mathrm{CSSiCl}$ & $110.6 \mathrm{~B}$ & $109.4 \mathrm{~B}$ \\
\hline
\end{tabular}

[1] R. Mockler, J. H. Bailey, and W. Gordy, J. Chem. Phys. 21, $1710(1953)$.

[2] M. Mitzlafi . R. Holm and H. Hartmann, Z. Naturforschg. 22a, 1415 (1967)

\section{Nitrogen trichloride}

$\mathrm{Cl}_{3} \mathrm{~N}$

\begin{tabular}{lll|lll} 
Bond Substitution Effective & Angle Substitution Effective \\
\hline $\mathrm{NCl}$ & $1.754 \mathrm{~A}$ & $1.759 \mathrm{~A}$ & $\mathrm{ClNCl}$ & $107.8 \mathrm{~A}$ & $107.4 \mathrm{~A}$
\end{tabular}

[1] G. Gazzoli, P. G. Favero, and A. Dal Borgo, J. Mol. Spectrosc. $50,82\{1974)$.

\section{Phosphoryl chloride}

\begin{tabular}{cc|cc}
$\mathrm{Cl}_{3} \mathrm{OP}$ & \\
\hline Bond & Effective & Angle & Substitution \\
\hline $\begin{array}{l}\mathrm{PO} \\
\mathrm{PCl}\end{array}$ & $\begin{array}{l}\mathrm{i} .455 \mathrm{n} \\
1.989 \mathrm{C}\end{array}$ & $\mathrm{ClPCl}$ & $103.7 \mathrm{C}$ \\
\hline
\end{tabular}

[1] Y. S. Li, M. M. Chen and J. R. Durig, J. Mol. Struct. 14, 261 (1972).

\section{Vanadyl(V) chloride}

\begin{tabular}{cc|cc}
$\mathrm{Cl}_{3} \mathrm{OV}$ & \\
\hline Bond & Effective & Angle & Effective \\
\hline $\mathrm{VO}$ & $1.587 \mathrm{C}$ & $\mathrm{CVCl}$ & $111.6 \mathrm{~B}$ \\
$\mathrm{VCl}$ & $2.133 \mathrm{~B}$ & & \\
\hline
\end{tabular}

The structural parameters were recalculated from the reported rotational constants.

[1] K. Karakida and K. Kuchitsu, Chem. Lett. 293 (1972).

\section{Phosphorous Trichloride}

\begin{tabular}{ll|ll}
$\mathrm{Cl}_{3} \mathrm{P}$ & \multicolumn{2}{c}{$\mathrm{PCl}_{\mathrm{3}}$} & $\mathrm{C}_{3 \mathrm{~V}}$ \\
\hline Bond & Effective & Angle & Effective \\
\hline $\mathrm{PCl}$ & $2.043 \mathrm{~B}$ & $\mathrm{ClPCl}$ & $100.1 \mathrm{C}$ \\
\hline
\end{tabular}

[I] P. Kisliuk and C. H. Townes, J. Chem. Phys. 18, 1109 (1950).

\section{Cesium Hydroxide}

\begin{tabular}{ccc} 
CsHO & $\mathrm{CsOH}$ & $\mathrm{C}_{\text {soo }}$ \\
\hline Bond & Effective & Equilibrium \\
\hline CsO & $2.403 \mathrm{C}$ & $2.391 \mathrm{~A}$ \\
$\mathrm{OH}$ & $0.920 \mathrm{C}$ & $0.960 \mathrm{C}$
\end{tabular}

[1] D. R. Lide, Jr. and R. L. Kuczkowski, J. Chem. Phys. 46, 4768 (1967).

[2] D. R. Lide, Jr. and C. Matsumura, J. Chem. Phys. 50, 3080 (1969).

\section{Fluorogermane}

\begin{tabular}{|c|c|c|c|c|}
\hline $\mathrm{GeH}_{3}$ & & & & $\mathrm{C}_{3}$ \\
\hline Bond & Substitution & Effective & Angle & Effective \\
\hline $\begin{array}{l}\mathrm{CeF} \\
\mathrm{GeH}\end{array}$ & $1.730 \mathrm{E}$ & $1.522 \mathrm{D}$ & $\mathrm{HCOH}$ & $113.0 \mathrm{D}$ \\
\hline
\end{tabular}

[1] L. C. Krisher, J. A. Morrison, W. A. Watson, J. Chem. Phys. 57, 1357 (1972).

[2] K. H. Rhee and M. K. Wilson, J. Chem. Phys. 43, 333 (1965). 


\section{Fluoroamidogen}

\begin{tabular}{ll|ll} 
FIIN & ANF & Angle & Effective \\
Bond & Effective & HNF & $105 \mathrm{C}$
\end{tabular}

Ground eleclronic state is " $A$ ".

NH distance assumed (1.06 $\mathrm{h}$ ).

[1] C. M. Woodman, J. Mol. Spectrosc. 33, 311 (1970).

\section{Hypofluorous acid}

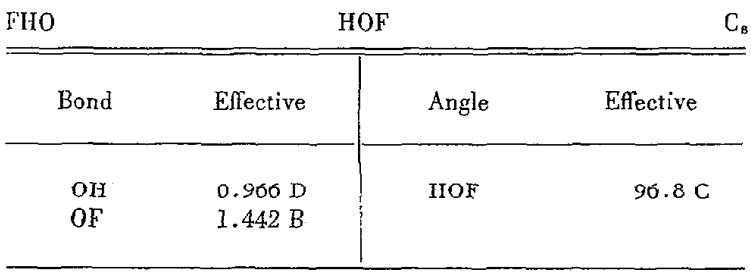

[1] E. F. Pearson and H. Kim, J. Chem. Phys. 57, 4230 (1972)

[2] H. Kim, E. F. Pearson and E. H. Appelman, J. Chem. Phys. 56,1 (1972).

\section{Fluorosilane}

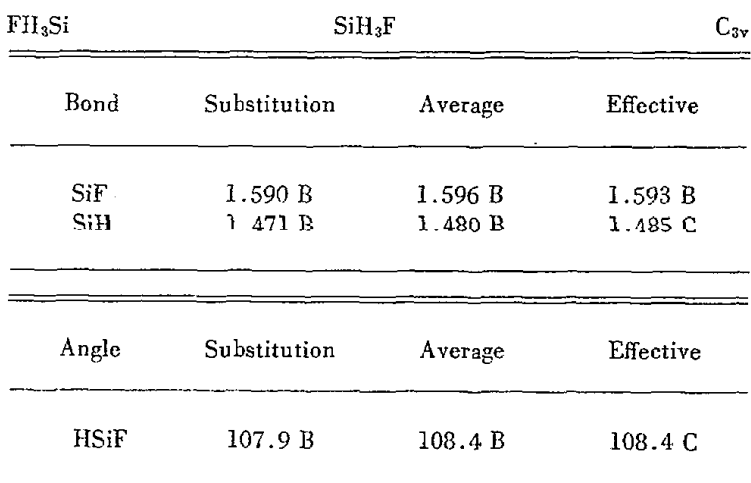

[1] A. H. Sharbaugh, V. G. Thomas and B. S. Pritchard, Phys Rev. 78, 64 (1950).

[2] B. Bak, J. Bruhn and J. Rastrup-Andersen, J. Chem. Phys. 21, 752 (1953).

[3] C. Georghiou, J. G. Baker and S. R. Jones, J. Mol. Spectrose. 63, 89 (1976).

[4.] A. G. Robiette, C. Georghiou and J. G. Baker, J. Mol. Spectrosc. $63,391(1976)$. $C_{B}$
Fluorodisilane

\begin{tabular}{|c|c|c|c|c|}
\hline $\mathrm{FH}_{5} \mathrm{Si}_{2}$ & & ${ }_{3} \mathrm{Si}-\mathrm{SiH}_{2} \mathrm{~F}$ & & $\mathrm{C}_{6}$ \\
\hline Bond & Substitution & Effective & Angle & Effective \\
\hline $\mathrm{SiSi}$ & $2.332 \mathrm{~B}$ & & SiSiF & $109.5 \mathrm{X}$ \\
\hline SiF & & $1.598 X^{x}$ & $\operatorname{SiSiH}(F)^{-}$ & $110.4 \mathrm{X}$ \\
\hline
\end{tabular}

Effective parameters determined by fitting momental equations along with the assumptions: $\mathrm{Si}-\mathrm{H}(F)=1.477, \mathrm{SiH}=1.483$, $\mathrm{HSiH}(\mathrm{F})=110^{\circ}, \mathrm{HSiH}=108.3^{\circ}$.

a $\mathrm{SiSiH}$ angle in $\mathrm{SiH}_{2} \mathrm{H}^{\prime}$ groups.

[1] A. P. Cox and R. Varma, J. Chem. Phys. 44, 2619 (1966).

\begin{tabular}{llll} 
BNo & Substitution & Angle & Substitution \\
NO & $1.136 \mathrm{~B}$ & FNO & $110.1 \mathrm{~B}$ \\
NF & $1.512 \mathrm{~B}$ & & \\
\hline
\end{tabular}

[1] K. S. Buckton, A. C. Legon and D. J. Miller, Trans. Faraday Soc. 65, 1975 (1969).

[2] R. L. Cuok, J. Chem. Phys. 42, 2927 (1965).

[3] A. Guarnieri, G. Zuliani and P. G. Favero, Nuovo Cimenti 39,76 (1965).

\section{Nitryl Fluoride}

\begin{tabular}{ll|cc}
$\mathrm{FNO}_{2}$ & & Enfective \\
\hline Bond & Effective & Angle & $136 \mathrm{D}$ \\
$\mathrm{NF}$ & $1.467 \mathrm{D}$ & $0 \mathrm{C} 0$ & \\
$\mathrm{NO}$ & $1.180 \mathrm{C}$ & & \\
\hline The oxygen coordinates were substitution coordinates.
\end{tabular}

[1] A. C. Legon and D. J. Millen, J. Chem. Soc. (A), 1736 (1968).

\section{Thiazyl fluoride}

\begin{tabular}{ll|ll} 
BNS & NSF & Angle & Effective \\
BS & $1.448 \mathrm{C}$ & NSF & $116.9 \mathrm{C}$ \\
$\mathrm{SF}$ & $1.643 \mathrm{C}$ & & \\
\hline
\end{tabular}

[1] R. L. Cook and W. H. Kirchhoff, J. Chem. Phys. 47, 4521 (1967)

[2] W. H. Kirchhoff and E. B. Wilson, J. Amer. Chem. Soc. 85, 1726 (1963). 


\section{Fluorotrioxorhenium}

\begin{tabular}{cc|cc} 
FO ${ }_{3}$ Re & \multicolumn{2}{c}{$\mathrm{FReO}_{3}$} & $\mathrm{C}_{3 \%}$ \\
\hline Bond & Effective & Angle & Effective \\
\hline $\begin{array}{ll}\text { ReO } \\
\text { ReF }\end{array}$ & $\begin{array}{l}1.692 \mathrm{~B} \\
1.859 \mathrm{C}\end{array}$ & FReO & $109.5 \mathrm{C}$
\end{tabular}

[1] J. F. Lotspeich, A. Javan, and A. Englebrecht, J. Chem. Phys. 31,633 (1959).

\section{Cermanium difluoride}

\begin{tabular}{cc|cc}
$\mathrm{F}_{2} \mathrm{Ge}$ & $\mathrm{FGeF}$ & Angle & Equitibrium \\
Bond & Equilibrium & $\mathrm{C}_{27}$ \\
$\mathrm{GeF}$ & $1.732 \mathrm{~A}$ & $\mathrm{FGeF}$ & $97.2 \mathrm{~A}$ \\
\hline
\end{tabular}

Equilibrium structure identical for three Ge isotopic species.

[1] H. Takeo and R. F. Curl, Jr., J. Mol, Spectrose, 43, 21 (1972).

[2] H. Takeo, R. F. Curl, Jr., and P. W. Wilson, J. Mol. Spectrosc, 38, 464 (1971).

\section{Fluorimide \\ (Difluoramine)}

$\mathrm{F}_{2} \mathrm{HN}$

\begin{tabular}{cc|cc}
\hline Bond & Effective & Angle & Effective \\
\hline $\mathrm{NF}$ & $1.400 \mathrm{~B}$ & FNF & $\begin{array}{r}102.9 \mathrm{~B} \\
\mathrm{NH}\end{array}$ \\
& $1.026 \mathrm{~B}$ & $\mathrm{HNF}$ & $99.8 \mathrm{~B}$ \\
\hline
\end{tabular}

[1] D. R. Lide, Jr., J. Chem. Phys. 38, 456 (1963):

[2] S. Sundaram, Proc. Phys. Soc. 92, 261 (1967).

\section{Difuvorophosphine}

$\mathrm{F}_{2} \mathrm{HP}$

\begin{tabular}{cc|cc}
\hline Bond & Effective & Angle & Effective \\
\hline $\mathrm{PF}$ & $1.582 \mathrm{~A}$ & $\mathrm{FPE}$ & $99.0 \mathrm{~A}$ \\
$\mathrm{PH}$ & $1.412 \mathrm{C}$ & $\mathrm{HPF}$ & $96.3 \mathrm{~B}$ \\
\hline
\end{tabular}

[1] R. I. Kuczkowski, J. Amer. Chem. Soc. 90, 1705 (1968).
Difluorophosphine Oxide

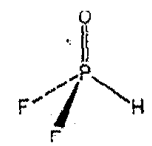

$\mathrm{F}_{\mathrm{2}} \mathrm{HPO}$

\begin{tabular}{cc|cr}
\hline Bond & Effective & Angle & Effective \\
\hline & & & \\
PH & $1.387 \mathrm{D}$ & HPO & $117.9 \mathrm{D}$ \\
PF & $1.539 \mathrm{~B}$ & FPO & $116.3 \mathrm{C}$ \\
PO & $1.437 \mathrm{~B}$ & FPF & $99.8 \mathrm{~B}$ \\
\hline
\end{tabular}

[1] L. F. Centofanti and R. L. Kuczkowski, Inorg. Chem. 7, 2582 (1968).

Hydrothiophosphoryl dinuoride

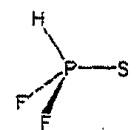

$\mathrm{F}_{2} \mathrm{HPS}$

\begin{tabular}{cc|cr}
$\mathrm{F}_{2} \mathrm{HPS}$ & & \\
\hline Bond & Effective & Angle & Effective \\
& & & \\
$\mathrm{PH}$ & $1.392 \mathrm{C}$ & $\mathrm{SPF}$ & $117.4 \mathrm{~B}$ \\
$\mathrm{PF}$ & $1.551 \mathrm{C}$ & $\mathrm{SPH}$ & $119.2 \mathrm{C}$ \\
$\mathrm{PS}$ & $1.867 \mathrm{C}$ & $\mathrm{FPF}$ & $98.6 \mathrm{~B}$ \\
\hline
\end{tabular}

[1] C. R. Nave and J. Sheridan, J. Mol. Struct. 15, 391 (1973)

Hydrogen fluoride dimer

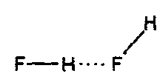

$\mathrm{F}_{2} \mathrm{H}_{2}$

$\mathrm{C}_{\mathrm{a}}$

Spectra consistent with bent model. With some assumptions, FF distance found to be $2.79 \hat{A}$.

[1] T. R. Dyke, B. J. Howard and W. Klemperer, J. Chem. Phys. 56,2442 (1972). 
Phosphoramidous difluoride

(Aminodifluorophosphine)

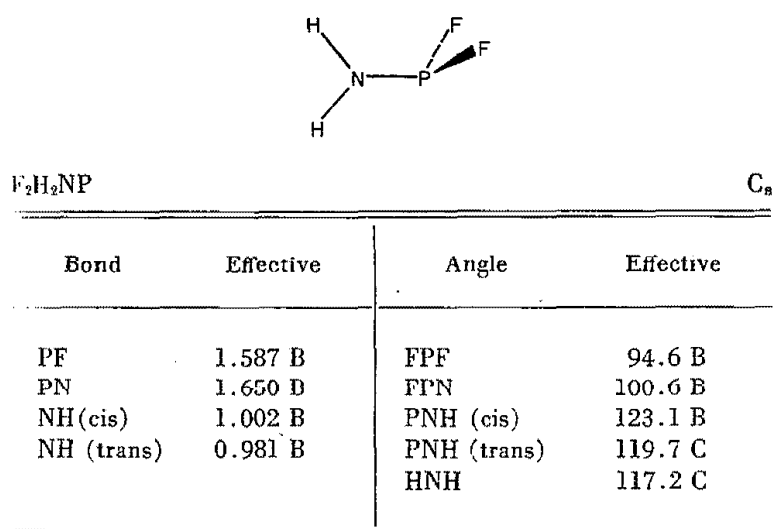

[1] A. H. Brittain, J. E. Smith, P. L. Lee, K. Cohn and R. H. Schwendeman, J. Arn. Chem. Soc. 98, 6772 (1971).

Phosphinodifluorophosphine

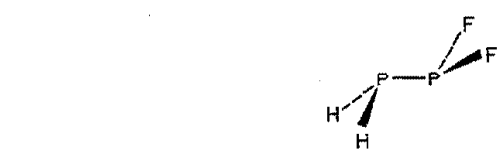

$\mathrm{F}_{2} \mathrm{H}_{2} \mathrm{P}_{2}$

\begin{tabular}{cc|cc}
\hline Bond & Effective & Angle & Effective \\
\hline & & & \\
$\mathrm{PP}$ & $2.218 \mathrm{X}$ & $\mathrm{FPF}$ & $98.2 \mathrm{X}$ \\
$\mathrm{PF}$ & $1.587 \mathrm{X}$ & $\mathrm{PPF}$ & $97.2 \mathrm{X}$ \\
& & $\mathrm{HPH}$ & $93.2 \mathrm{X}$ \\
& & $\mathrm{PPH}$ & $90.3 \mathrm{X}$ \\
\hline
\end{tabular}

The PH distance has been assumed to be $1.42 \AA$.

[1] R. L. Kuczkowski, H. W. Schiller and R. W. Rudolph, Inorg Chem. 10, 2505 (1971).

\section{Krypton Difluoride}

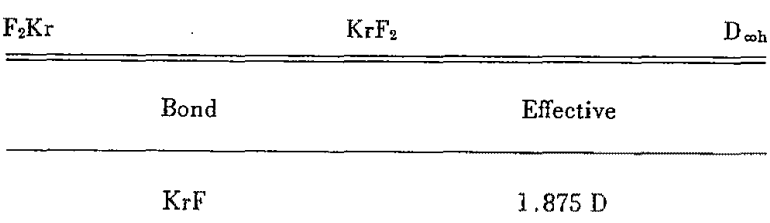

Two possithe $I$ assignments of the $x_{i}$ band of KrF exist. The bond distance cited above is that calculated from the $B_{0}$ value from the most probable assignment. The error taken, however, is that which will encompass both possible assignments.

[1] C. Murchison, S. Reichman, D. Anderson, J. Overend and F. Schreiner, J. Am. Chem. Soc. 90, 5690 (1968).
Difluoroamidogen

(Nilrogen difluoride)

$\mathrm{F}_{2} \mathrm{~N}$

\begin{tabular}{lll|lll}
\hline \hline Bond & Effective & Average & Angle & Effective & Average \\
\hline $\mathrm{NF}$ & $1.349 \mathrm{~B}$ & $1.353 \mathrm{~B}$ & $\mathrm{FNF}$ & $103.3 \mathrm{~B}$ & $103.2 \mathrm{~B}$ \\
\hline
\end{tabular}

Ground state is ${ }^{2} \mathrm{~B}_{1}$.

[1] R. D. Brown, F. R. Burden, P. D. Godfrey and J. R. Gillard, J. Mol. Spectrose. 25, 301 (1974).

\section{cis-Difluorodiazine}

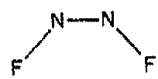

$\mathrm{F}_{2} \mathrm{~N}_{3}$

\begin{tabular}{|c|c|c|c|c|}
\hline Bond & Substitution & Effective & Angle & Effective \\
\hline $\begin{array}{l}\text { NN } \\
\text { NF }\end{array}$ & $1.214 \mathrm{~B}$ & $1.385 \mathrm{~B}$ & FNN & $114.6 \mathrm{~B}$ \\
\hline
\end{tabular}

[1] R. L. Kuczkowski and E. B. Wilson, Jr., J. Chem. Phys. 39, $1030(1963)$.

\section{Oxygen Difluoride}

$\mathrm{F}_{2} \mathrm{O}$

$\mathrm{C}_{2 \mathrm{v}}$

\begin{tabular}{cccc}
\hline Bond & Effective & Average & Equilibrium \\
\hline OF & $1.409 \mathrm{~A}$ & $1.412 \mathrm{~A}$ & $1.405 \mathrm{~A}$ \\
\hline \hline Angle & Effective & Average & Equilibrium \\
\hline FOF & $103.3 \mathrm{~A}$ & $103.2 \mathrm{~A}$ & $103.1 \mathrm{~A}$ \\
\hline
\end{tabular}

Studies in the ground and excited states permitted determination of effective, average and equilibrium structures.

[1] L. Pierce, N. DiCianni, and R. H. Jackson, J. Chem. Phys. 38, 730 (1963).

[2] Y. Morino and S. Saito, J. Mol. Spectrosc. 19, 435 (1966). 


\section{Thionyl Fluoride}

\begin{tabular}{lll|lrr}
$\mathrm{F}_{2} \mathrm{OS}$ & \multicolumn{2}{c}{$\mathrm{SOF}_{2}$} & $\mathrm{C}_{\mathrm{S}}$ \\
Bond & Effective & Average & Angle & Effective & Average \\
\hline SO & $1.413 \mathrm{~A}$ & $1.416 \mathrm{~A}$ & $\mathrm{FSF}$ & $92.83 \mathrm{~A}$ & $92.79 \mathrm{~A}$ \\
$\mathrm{SF}$ & $1.585 \mathrm{~A}$ & $1.587 \mathrm{~A}$ & $\mathrm{FSO}$ & $106.82 \mathrm{~A}$ & $106.66 \mathrm{~A}$ \\
\hline
\end{tabular}

[1] R. C. Ferguson, J. Am. Chem. Soc. 76, 850 (1951).

[2] N. J. D. Lucas and J. G. Smith, I. Mol. Spectroso, 43,327 (1972).

\section{Seleninyl Fluoride}

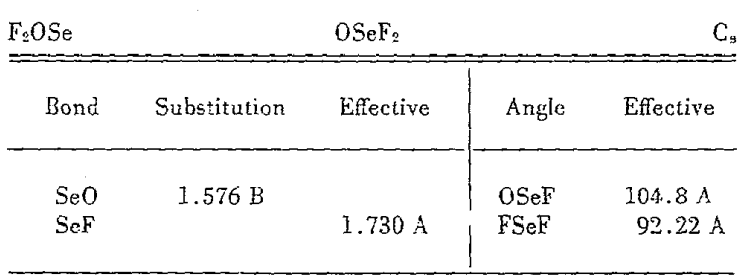

[1] 1. C. Bowater, R. D. Brown, and F. R. Burden, J. Mol. Spectrose. 28,461 (1968).

\section{Dioxygen Difluoride}

\begin{tabular}{|c|c|c|c|c|}
\hline Bond & Substitution & Effective & Angle & Effective \\
\hline 00 & $1.217 \mathrm{~B}$ & & OOF & $109.5 \mathrm{~B}$ \\
\hline$O F$ & & $1.575 \mathrm{~B}$ & $\varphi^{\mathrm{a}}$ & $87.5 \mathrm{~B}$ \\
\hline
\end{tabular}

aihedral angle.

[1] R. H. Jackson, J. Chem. Soc. 884, 4585 (1962).

Sulfuryl Fluoride

\begin{tabular}{cc|cc}
$\mathrm{F}_{2} \mathrm{O}_{2} \mathrm{~S}$ & \multicolumn{2}{c}{$\mathrm{F}_{2} \mathrm{SO}_{2}$} & $\mathrm{C}_{2 v}$ \\
\hline Bond & Effective & Angle & Effective \\
\hline $\mathrm{SO}$ & $1.405 \mathrm{C}$ & 050 & $124.0 \mathrm{C}$ \\
$\mathrm{SF}$ & $1.530 \mathrm{C}$ & FSF & $96 . \mathrm{C} \mathrm{C}$ \\
\hline
\end{tabular}

[1] D. R. Lide, D. E. Mann and R. M. Fristom, J. Chen. Phys. 26,734 (1957).

3. Fhys. Chem. Ref. Data, Vol. 8, No. 3, 1979
Sulfur Difluoride

\begin{tabular}{lll|lll}
$\mathrm{F}_{2} \mathrm{~S}$ & & $\mathrm{C}_{2 \mathrm{~V}}$ \\
\hline Bond & Effective & Average & Angle & Effective & Average \\
\hline $\mathrm{SF}$ & $1.589 \mathrm{G}$ & $1.592 \mathrm{~A}$ & $\mathrm{FSF}$ & $98.3 \mathrm{~B}$ & $98.2 \mathrm{~A}$ \\
\hline
\end{tabular}

[1] D. R. Johnson and F. X. Powell, Science 164, 950 (1969).

[2] W. H. Kirchhof, D. R. Johnson, and F. X. Powell, J. Mol. Spectrosc. 48, 157 (1973).

\section{Thionyl Fluoride}

$\mathrm{F} .5 \mathrm{O}$

$\mathrm{C}_{5}$

\begin{tabular}{lll|lrr}
\hline Bond & Effective & Average & Angle & Effective & Average \\
& & & & & \\
SO & $1.413 \mathrm{~B}$ & $1.416 \mathrm{~B}$ & FSF & $92.8 \mathrm{~B}$ & $92.8 \mathrm{~B}$ \\
SF & $1.585 \mathrm{~B}$ & $1.587 \mathrm{~B}$ & FSO & $106.8 \mathrm{~B}$ & $106.7 \mathrm{~B}$ \\
& & & & &
\end{tabular}

[1] N. J. D. Lucas and J. G. Smith, J. Mol. Spectrose. 43, 327 (1972).

[2] R. C. Ferguson, J. Ame:. Chem. Soc. 76, 850 (1954).

Disulfur Difluoride (Sulfur Monofluoride Dimer)

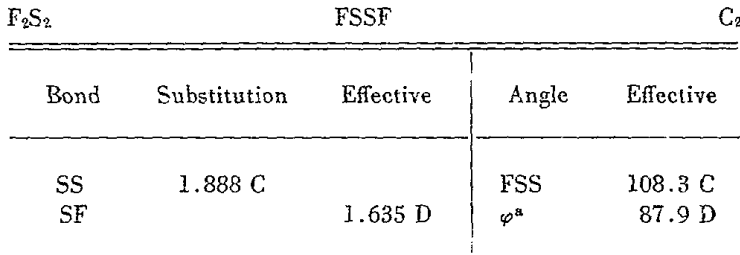

a Dihedral angle.

[1] R. L. Kuczkowshi, J. Amer. Chem. Soc. 86, 3617 (1964).

Disulfur Difluoride (Thiothionylfluoride)

\begin{tabular}{|c|c|c|c|c|}
\hline Bond & Substitution & Effective & Angle & Effective \\
\hline SS & $1.860 \mathrm{D}$ & & SSF & $107.5 \mathrm{C}$ \\
\hline $\mathrm{SF}$ & & 1.5980 & FSF & $92.5 \mathrm{C}$ \\
\hline
\end{tabular}

One sulfur atom has rather small $a$ and $e$ coordinates, and the sccond has a amall o coordinate, which degrades the $r_{\mathrm{s}} \mathrm{S} S$ distance. [1] R. L. Kuczkowski, J. Amer. Chem. Soc. 86, 3617 (1964). 
Difluorosilylene

(Silicon difluoride)

\begin{tabular}{lll|lll} 
Bond & Effective & Equilibrium & Angle & Effective & Equilibrium \\
\hline $\mathrm{SiF}$ & $1.591 \mathrm{~A}$ & $1.590 \mathrm{~A}$ & FSiF & $100.0 \mathrm{~A}$ & $100.8 \mathrm{~A}$
\end{tabular}

[1] H. Shoji, T. Tanaka and E. Hirota, J. Mol. Spectrose. 47, 268 (1973).

[叉] V. M. Rao, R. F. Curl, Jr., P. L. Timms and J. L. Margrave, J. Chem. Phys. 43, 2557 (1065).

\section{Xenon Difluoride}

$\mathrm{r} \times \mathrm{Xe}$

\begin{tabular}{c|c}
\hline Bond & Efective \\
\hline $\mathrm{Xe}^{-\mathrm{F}}$ & $1.977 \mathrm{~B}$ \\
\hline
\end{tabular}

[1] S. Reichman and F. Sohreizer, J. Chem. Phye. 51, 2355 (1960).

Trifluorosilane

\begin{tabular}{lll|lll}
$\mathrm{F}_{3} \mathrm{HSi}$ & & & $\mathrm{C}_{3 \mathrm{v}}$ \\
\hline \hline Bond & Equilibrium & Effective & Angle & Equilibrium & Effective \\
\hline $\mathrm{SiF}$ & $1.562 \mathrm{~A}$ & $1.564 \mathrm{~B}$ & $\mathrm{FSiF}$ & $108.3 \Lambda$ & $108.3 \mathrm{~B}$ \\
$\mathrm{SiH}$ & $1.447 \mathrm{~A}$ & $1.455 \mathrm{C}$ & & &
\end{tabular}

[1] A. R. Hoy, M. Bertram and I. M. Mills, J. Mol. Spectrosc. 46, 429 (1973).

[2] G. A. Heath, L. F. Thomas and J. Sheridan, Trans. Faraday Soc. 50. 779 (1954).

[3] J. Sheridan and W. Gordy, J. Chem. Phys. 19, 965 (1951).

\section{1,1,1-Trifluorodisilane}

\begin{tabular}{lll|ll}
$\mathrm{F}_{3} \mathrm{H}_{3} \mathrm{Si}_{2}$ & \multicolumn{2}{c}{$\mathrm{F}_{3} \mathrm{Si}_{-} \mathrm{SiH}_{3}$} & & $\mathrm{C}_{35}$ \\
\hline Bond & Substitution & Effective & Angle & Effective \\
\hline SiSi & $2.319 \mathrm{~B}$ & & SiSir & $108.7 \mathrm{X}$ \\
$\mathrm{Sill}$ & & $\mathrm{I} .480^{\mathrm{a}}$ & $\mathrm{SiSiF}$ & $112.0 \mathrm{X}$ \\
$\mathrm{SiF}$ & & $1.561^{\circ}$ & & \\
\hline
\end{tabular}

assumed values.

[1] J. Pasinski, S. A. MeMrahon and R. A. Beaudet, J. Mol. Spec trose. 55,88 (1975).
Trifluoroiodosilane

$\mathrm{F}_{3} \mathrm{ISi}$

$\mathrm{F}_{3} \mathrm{SiI}$

$\mathrm{C}_{3 \mathrm{r}}$

\begin{tabular}{cc}
\hline Bond & Effective \\
\hline Sil & $2.387 \mathrm{D}$
\end{tabular}

SiF bond and FSiF angle were aseumod.

[1] L. C. Sams, Jr., and A. W. Jache, J. Chem. Phys. 47, 1314, (1967).

\section{Nitrogen Trifluoride}

$\mathrm{F}_{3} \mathrm{~N}$

\begin{tabular}{lll|lll}
\hline Bond & Effective & Equilibrium & Angle & Effective & Equilibrium \\
\hline NF & $1.371 \mathrm{~A}$ & $1.365 \mathrm{~A}$ & FNF & $102.2 \mathrm{~A}$ & $102.4 \mathrm{~A}$ \\
\hline
\end{tabular}

[1] J. Sheridan and W. Gordy, Phys. Rev. 79, 513 (1950)

[2] M. Otake, C. Matsumura, and Y. Morino, J. Mol. Spectrosc. $28,316(1068)$

\section{Nitrogen Sulfur Trifluoride}

\begin{tabular}{ccc|cc}
$\mathrm{H}_{3} \mathrm{NS}$ & & $\mathrm{NSF}_{3}$ & & $\mathrm{C}_{3 \mathrm{v}}$ \\
\hline \hline Bond & Substitution & Effective & Angle & Effective \\
\hline $\begin{array}{c}\mathrm{NS} \\
\mathrm{SF}\end{array}$ & $1.416 \mathrm{C}$ & & FSF & $94.0 \mathrm{~B}$ \\
\hline
\end{tabular}

[1] W. H. Kirchhoff and E. B. Wilson, Jr., J. Amer. Chem. Soc. 84, 334. (1962).

\section{Phosphoryl Fluoride}

\begin{tabular}{ll|ll}
$\mathrm{F}_{3} \mathrm{OP}$ & \multicolumn{1}{c}{$\mathrm{OPF}_{3}$} & $\mathrm{C}_{3 \mathrm{v}}$ \\
\hline \hline Bond & Effective & Angle & Effective \\
\hline $\mathrm{PO}$ & $1.437 \mathrm{~A}$ & $\mathrm{FPF}$ & $101.14 \mathrm{~A}$ \\
$\mathrm{PF}$ & $1.522 \mathrm{~A}$ & & \\
\hline
\end{tabular}

[1] R. H. Kagann, I. Oxier, and M. C. L. Gerry, Chem. Phys. Lett, $47572(197 \%)$.

[2] J. G. Snith, Mol. Phys. 32, 621 (1976). 


\section{Phosphorus trifluoride}

\begin{tabular}{cccc}
\hline Bond & Equilibrium & Average & Effective \\
\hline PF & $1.561 \mathrm{~A}$ & $1.565 \mathrm{~A}$ & $1.563 \mathrm{~A}$ \\
\hline & & & $\mathrm{C}_{3 \mathrm{v}} \mathrm{P}$ \\
\hline Angle & Equilibrium & Average & Effective \\
\hline FPF & $97.7 \mathrm{~A}$ & $97.6 \mathrm{~A}$ & $97.7 \mathrm{~A}$
\end{tabular}

[1] Y. Kawashima and A. I. Cox, J. Mol. Spectrosc. 65, 319 (1977).

[2] E. Hirota and Y. Morino, J. Mol. Spectrose. 33, 460 (1970).

\section{Thiophosphoryl Fluoride}

\begin{tabular}{cc|cc}
\multicolumn{2}{l}{$\mathrm{F}_{3} \mathrm{PS}$} & & $\mathrm{C}_{3 v}$ \\
\hline Bond & Effective & Angle & Effective \\
\hline PS & $\begin{array}{l}1.87 \mathrm{E} \\
\text { PF }\end{array}$ & FPF & $100.3 \mathrm{E}$
\end{tabular}

[1] Q. Williams, J. Sheridan and W. Gordy, J. Chem. Phys. 20, 164. (1952).

\section{Tetrafluorophosphorane}

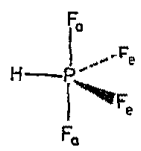

\begin{tabular}{cc|cc}
$\mathrm{F}_{4} \mathrm{HP}$ & \\
\hline Bond & Efective & Angle & Effective \\
\hline $\mathrm{PF}_{\mathrm{a}}$ & $1.596 \mathrm{~B}$ & $\mathrm{CPF}_{2 \mathrm{v}}$ & $90 \mathrm{E}$ \\
$\mathrm{PF}_{\mathrm{a}}$ & $1.55 \mathrm{E}$ & $\mathrm{HPF}_{*}$ & $124 \mathrm{D}$ \\
\hline
\end{tabular}

The $\mathrm{PH}$ distance was assumed.

[1] S. B. Pierce and C. D. Cornwell, J. Chem. Phys. 48, 2118 (1968).

\section{Xenon Tetrafluoride Oxide}

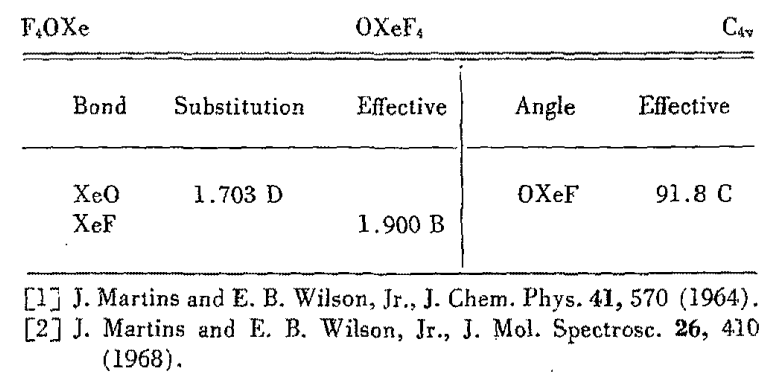

\section{Sulfur Tetrafluoride}

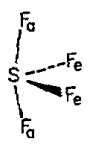

F\&s

\begin{tabular}{cc|cc}
\hline Bond & Effective & Angle & Effective \\
\hline & & & \\
$\mathrm{SF}_{2}$ & $1.646 \mathrm{~B}$ & $\mathrm{~F}_{\mathrm{SF}}$ & $101.5 \mathrm{C}$ \\
$\mathrm{SF}_{\mathrm{e}}$ & $1.545 \mathrm{~B}$ & $\mathrm{~F}_{\mathrm{s}} \mathrm{SF}_{\mathrm{a}}$ & $186.9 \mathrm{C}$ \\
\hline
\end{tabular}

The sulfur coordinate was a substitution coordinate.

[1] W. M. Tolles and W. D. Gwinn, J. Chem. Phys. 36, 1119 (1962).

Selenium Tetrafluoride

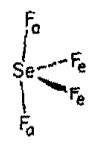

$\mathrm{F}_{4} \mathrm{Se}$

\begin{tabular}{cc|cc}
\hline Bond & Effective & Angle & Effective \\
\hline & & & \\
$\mathrm{SeF}_{\mathrm{n}}$ & $1.771 \mathrm{C}$ & $\mathrm{F}_{\mathrm{Q}} \mathrm{SeF}_{\mathrm{g}}$ & $100.5 \mathrm{C}$ \\
$\mathrm{SeF}_{\mathrm{e}}$ & $1.682 \mathrm{C}$ & $\mathrm{F}_{\mathrm{l}} \mathrm{SeF}_{\mathrm{a}}$ & $169.2 \mathrm{C}$ \\
\hline
\end{tabular}

The selenium coordinate was a substitution coordinate.

[1] 1. L. Bowater, K. D. Brown, and H. K. Burden, J. Mol. Spectrosc. 28, 454 (1968).

\section{lodogermane}

\begin{tabular}{|c|c|}
\hline $\mathrm{GeH}_{3} \mathrm{I}$ & $\mathrm{IGeH}_{3}$ \\
\hline Bond & Effective \\
\hline GeI & $2.508 \mathrm{~B}$ \\
\hline
\end{tabular}

$\mathrm{H}_{3} \mathrm{Ge}$ projection on $\mathrm{C}_{3}$ axis was assumed.

[1] S. N. Wolf and L. C. Krisher, J. Chem. Phys. 56, 1040 (1972). 


\section{Germane}

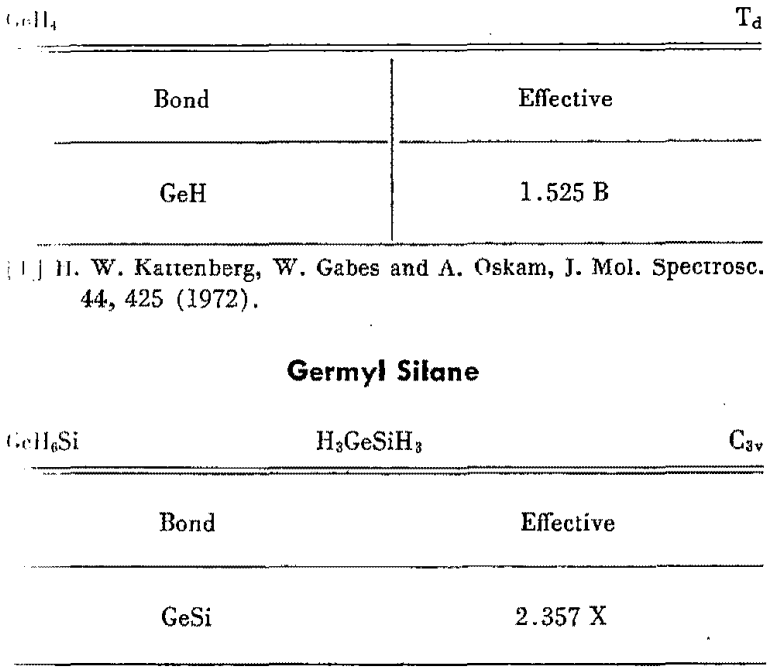

Parameters for the $\mathrm{GeH}_{3}$ and $\mathrm{SiH}_{3}$ groups were assumed.

[1] A. P. Cox and R. Varma, J. Chem. Phys. 46, 1603 (1967).

\begin{tabular}{lc|ccc}
\multicolumn{3}{c}{ lodosilylene } \\
HISi & HSiI & $\mathrm{C}_{B}$ \\
\hline Bond & Effective & Angle & Effective \\
\hline Sil & $2.451 \mathrm{~B}$ & HSiI & $102.7 \mathrm{C}$ \\
\hline
\end{tabular}

SiH distance assumed (1.561 $\AA$ ).

[1] J. Billingsley, Can. J. Phys. 50, 531 (1972).

\section{Potassium hydroxide}

\begin{tabular}{ll} 
HKO & $\mathrm{KOH}$ \\
\hline \hline Bond & Effective \\
\hline $\mathrm{KO}$ & $2.212 \mathrm{X}$ \\
$\mathrm{OH}$ & $0.912 \mathrm{X}$
\end{tabular}

The molecule has been assumed to be linear.

[1] E. F. Pearson and M. B. Trueblood, J. Chem. Phys. 58, 826 (1973).

\section{Nitrosyl Hydride}

HNO

\begin{tabular}{cc|cc}
\hline Bond & Effective & Angle & Effective \\
\hline NH & $1.063 \mathrm{C}$ & HNO & $108.6 \mathrm{~B}$ \\
NO & $1.212 \mathrm{~A}$ & & \\
\hline
\end{tabular}

[1] F. W. Dalby, Can. J. Phys. 36, I336 (1958).
cis-Thionylimide

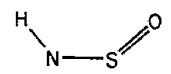

HNOS

$\mathrm{C}_{s}$

\begin{tabular}{ll|ll}
\hline \hline Bond & Substitution & Angle & Substitution \\
\hline NH & $1.029 \mathrm{C}$ & HNS & $115.8 \mathrm{C}$ \\
NS & $1.512 \mathrm{C}$ & NSO & $120.4 \mathrm{C}$ \\
SO & $1.451 \mathrm{C}$ & & \\
\hline
\end{tabular}

[1] W. H. Kirchhoff, J. Amer. Chem. Soc. 91, 2437 (1969).

\section{cis-Nitrous Acid}

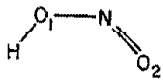

$\mathrm{HNO}_{2}$

$\mathrm{C}_{*}$

\begin{tabular}{ll|ll}
\hline Bond & Effective & Angle & Effective \\
\hline $\mathrm{HO}_{1}$ & $0.982 \mathrm{D}$ & $\mathrm{O}_{1} \mathrm{NO}_{2}$ & $113.6 \mathrm{D}$ \\
$\mathrm{NO}_{1}$ & $1.392 \mathrm{D}$ & $\mathrm{HO}_{1} \mathrm{~N}$ & $104.0 \mathrm{D}$ \\
$\mathrm{NO}_{2}$ & $1.185 \mathrm{D}$ & & \\
\hline
\end{tabular}

[I] A. P. Cox, A. H. Brittain, and D. J. Finnegan, Trans. Faraday Soc. 67,2179 (1971).

\section{trans-Nitrous Acid}

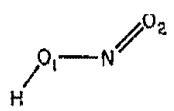

$\mathrm{HNO}_{2}$

\begin{tabular}{cc|cc}
\hline \hline Bond & Substitution & Angle & Substitution \\
\hline & & & \\
$\mathrm{H}-\mathrm{O}_{2}$ & $0.958 \mathrm{C}$ & $\mathrm{O}_{1} \mathrm{NO}_{2}$ & $110.7 \mathrm{C}$ \\
$\mathrm{N}-\mathrm{O}_{1}$ & $1.432 \mathrm{C}$ & $\mathrm{HO}_{1} \mathrm{~N}$ & $102.1 \mathrm{C}$ \\
$\mathrm{N}-\mathrm{O}_{2}$ & $1.170 \mathrm{C}$ & & \\
\hline
\end{tabular}

[1] A. P. Cox, A. H. Brittain and D. J. Finnegan, Trans. Faraday Soc. 67,2179 (1971).

[2] A. P. Cox and R. L. Kuczkowski, J. Amer. Chem. Soc. 88, $5071(1966)$ 
Nitric Acid

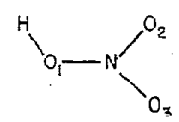

$\mathrm{HNO}_{3}$

\begin{tabular}{cc|cc}
\hline Bond & Substitution & Angle & . Substitution \\
\hline & & & \\
$\mathrm{HO}_{3}$ & $0.964 \mathrm{C}$ & $\mathrm{HO}_{1} \mathrm{~N}$ & $102.2 \mathrm{C}$ \\
$\mathrm{NO}_{1}$ & $1.406 \mathrm{C}$ & $\mathrm{O}_{1} \mathrm{NO}_{2}$ & $115.9 \mathrm{C}$ \\
$\mathrm{NO}_{2}$ & $1.211 \mathrm{C}$ & $\mathrm{O}_{1} \mathrm{NO}_{3}$ & $113.8 \mathrm{C}$ \\
$\mathrm{NO}_{3}$ & $1.199 \mathrm{C}$ & $\mathrm{O}_{2} \mathrm{NO}_{3}$ & $130.3 \mathrm{C}$ \\
\hline
\end{tabular}

[1] A. P. Cox and J. M. Riveros, J. Chem. Phys. 42, 3106 (1965).

[2] D. J. Millen and J. R. Morton, J. Chem. Soc. 1523 (1960).

\section{Hydrazoic Acid}

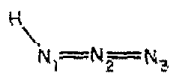

$\mathrm{HN}_{3}$

\begin{tabular}{ll|ll}
\hline Bond & Effective & Angle & Efective \\
\hline $\mathrm{N}_{1} \mathrm{H}$ & $0.975 \mathrm{X}$ & $\mathrm{HN}_{\mathrm{S}} \mathrm{N}_{2}$ & $114.1 \mathrm{X}$ \\
$\mathrm{N}_{1} \mathrm{~N}_{2}$ & $1.237 \mathrm{X}$ & & \\
$\mathrm{N}_{2} \mathrm{~N}_{3}$ & $1.133 \mathrm{X}$ & & \\
\hline
\end{tabular}

The $\mathrm{N}_{3}$ fragment was assumed to be linear.

[1] M. Winnewisser and R. L. Cook, J. Chem. Phys. 41, 999 (1964).

\begin{tabular}{cc|cc} 
Oxophosphine & Angle & Effective \\
\hline Bond & Effective & AnO & C \\
\hline PO & $1.512 \mathrm{~B}$ & HPO & $104.7 \mathrm{C}$ \\
\hline
\end{tabular}

PH dislance assumed (1.433 $\bar{\lambda})$.

[1] M. Lam Thanh and M. Peyron, J. Chem. Phys. 61, 1531 (1964).
Rubidium Hydroxide

\begin{tabular}{c|cc} 
HORZ & Bond \\
Effective & Equilibrium \\
RbO & RbOH & \\
OH & $2.316 \mathrm{C}$ & $2.301 \mathrm{~A}$ \\
& $0.913 \mathrm{C}$ & $0.957 \mathrm{C}$ \\
\hline
\end{tabular}

[1] C. Matsumura and D. R. Lide, Jr., J. Chem. Phys. 50, 71 (196.

[2] C. Matsumura and D. R. Lide, Jr., J. Chem. Phys. 50, 3C (1969).

\section{Hydroperoxyl radical}

$\mathrm{HO}_{2}$

\begin{tabular}{cc|cc} 
Bond & Effective & Angle & Effective \\
\hline HO & $\begin{array}{l}0.977 \mathrm{C} \\
00\end{array}$ & HoO & 104.I D \\
\hline
\end{tabular}

Ground state is ${ }^{2} \mathrm{~A}$ ".

[1] Y. Beers and C. J. Howard, J. Chem. Phys. 64, 1541 (1976),

[2] J. T. Hougen, H. E. Radford, K. M. Evenson, and C. J. Howar J. Mol. Spectrose. 56, 210 (1975).

Cs [3] Y. Beers and C. J. Howard, J. Chen. Phys. 63, 4212 (1975). [4] S. Saito, J. Mol. Spectrosc. 65, 229 (1977).

Hydrogen Krypton(1/1)

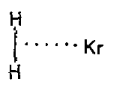

$\mathrm{H}_{2} \mathrm{Kr}$

Undefiner

\begin{tabular}{ll} 
Bond & Effective \\
\hline $\begin{array}{c}\text { Mean value between center of } \\
\text { mass of } \mathrm{H}_{2} \text { and } \mathrm{Kr} \text { atom }\end{array}$ & $4.07 \mathrm{X}$ \\
\hline
\end{tabular}

[1] A. R. W. McKellar and H. L. Welsch, J. Chem. Phys. 55, 595 (1971).

\section{Amidogen}

\begin{tabular}{cc|cc} 
Bond & Effective & Angle & Effective \\
\hline NH & $1.024 \mathrm{C}$ & HNH & $103.3 \mathrm{~B}$ \\
\hline
\end{tabular}

Ground electronic state is ${ }^{2} \mathrm{~B}_{1}$.

[1] K. Dressler and D. A. Ramsay, Phil. Trans. Roy. Soc. (London) A251, 553 (1959). 


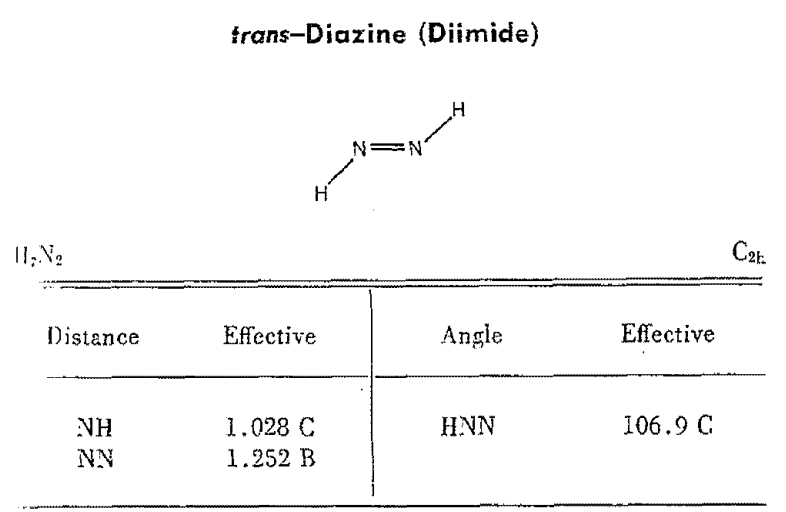

[1] M. Carlotti, J. W. C. Johns and A. Trombetti, Can. J. Phys. $\mathbf{5 2}, 340(1974)$.

\section{Nitramide}

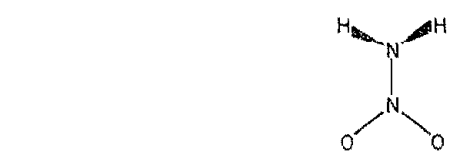

\begin{tabular}{cc|cr}
$\mathrm{U}_{2} \mathrm{~N}_{2} \mathrm{O}_{3}$ & & $\mathrm{C}_{s}$ \\
\hline Bond & Effective & Angle & Effective \\
\hline $\mathrm{NN}$ & $1.427 \mathrm{X}$ & $\mathrm{HNIl}$ & $115.2 \mathrm{X}$ \\
$\mathrm{NH}$ & $1.005 \mathrm{X}$ & ONO & $130.1 \mathrm{X}$ \\
& & $\varphi^{\mathrm{s}}$ & $51.8 \mathrm{X}$ \\
\hline
\end{tabular}

a Angle between $\mathrm{NH}_{2}$ and $\mathrm{NNO}_{2}$ planes.

[1] J. K. Tyler, J. Mol. Spectrosc. 11, 39 (1963).

\section{Hydrogen Neon(1/1)}

$$
\int_{H}^{H} \cdots \text { Ne }
$$

\begin{tabular}{l|c}
$\mathrm{H}_{2} \mathrm{Ne}$ & Undefined \\
\hline Bond & $3.99 \times$ \\
$\begin{array}{c}\text { Mean value between center of } \\
\text { mass of } \mathrm{H}_{2} \text { and Ne atom }\end{array}$ & \\
\hline
\end{tabular}

[I] A. R. W. MeKellar and H. L. Welsh, Can. J. Phys. 50, 1458 (1972).
Water

\begin{tabular}{|c|c|c|c|c|}
\hline Boncे & Effective & Substitution & Average & Equilibrium \\
\hline $\mathrm{OH}$ & $0.965 \mathrm{D}$ & $0.959 \mathrm{C}$ & $0.972 \mathrm{~A}$ & $0.958 \mathrm{~A}$ \\
\hline Angle & Effective & Substitution & Average & Equilibrium \\
\hline $\mathrm{HOH}$ & $104.8 \mathrm{D}$ & $104.5 \mathrm{C}$ & $104.5 \mathrm{~A}$ & $104.5 \mathrm{~A}$ \\
\hline
\end{tabular}

[1] R. L. Cook, F. C. DeLucie and P. Helminger, J. Mol. Spectrosc. 53. 62 (1974).

[2] W. S. Benedict, N. Gajlar, and E. K. Plyler, J. Chern. Phys. 24, 1139 (1956).

\section{Water +1 ) Ion}

$\mathrm{H}_{2} \mathrm{O}^{+}$

\begin{tabular}{cc|cc}
\hline Bond & Efective & Angle & Effective \\
\hline OH & $0.999 \mathrm{C}$ & HOH & $110.5 \mathrm{~B}$ \\
\hline
\end{tabular}

Ground electronic state is ${ }^{2} B_{1}$.

[1] H. Lew and I. Heiber, J. Chem. Phys. 58, 1246 (1973).

\section{Hydrogen Peroxide}

$\mathrm{H}_{2} \mathrm{O}_{2}$ $\mathrm{C}_{2}$

\begin{tabular}{c|c}
\hline Angle & Effective \\
\hline Dihedral & $120 \mathrm{X}$ \\
\hline
\end{tabular}

Average dihedral angle in the lowest $(\mathbb{N}=0, \tau=1,2)$ internal rotation states.

[1] W. C. Oelfke and W. Gordy, J. Chem. Phys. 5l, 5336 (1969).

\section{Phosphino}

\begin{tabular}{cc|cc}
$\mathrm{H}_{2} \mathrm{P}$ & & $\mathrm{C}_{2 \mathrm{v}}$ \\
\hline Bond & Effective & Angle & Effective \\
\hline $\mathrm{PH}$ & $1.418 \mathrm{C}$ & $\mathrm{HPH}$ & $91.7 \mathrm{~B}$ \\
\hline
\end{tabular}

Ground electronic state is ${ }^{2} \mathrm{~B}_{1}$.

[1] R. N. Dixon, G. Duxbury and D. A. Ramsay, Proc. Roy. Soe, (London) A296, 137 (1967).

[2] I. M. Berthou, B. Pascat, H. Guenebaut and D. A. Ramsay. Can. J. Phys. 50, 2265 (1972). 
Hydrogen Sulfide

\begin{tabular}{lll|lll}
\hline \hline $\mathrm{H}_{0} \mathrm{~S}$ & & $\mathrm{C}_{2 \mathrm{v}}$ \\
\hline Bond & Effective & Equilibrium & Angle & Effective & Substitute \\
\hline $\mathrm{HS}$ & $1.344 \mathrm{D}$ & $1.336 \mathrm{~A}$ & $\mathrm{HSH}$ & $92.2 \mathrm{D}$ & $92.1 \mathrm{~A}$ \\
\hline
\end{tabular}

[1] P. Helminger, R. L. Cook and F. DeLucia, J. Chem. Phys. 56, 4581 (1972).

[2] T. H. Edwards, N. K. Moncur and L. E. Snyder, J. Chem. Phys. 46, 2139 (1967).

\section{Hydrogen Sulfide $(+1)$ Ion}

$\mathrm{H}_{2} \mathrm{~S}^{+}$

\begin{tabular}{cc|cc}
\hline Bond & Effective & Angle & Effective \\
\hline $\mathrm{SH}$ & $1.358 \mathrm{C}$ & HSII & $92.9 \mathrm{D}$ \\
\hline
\end{tabular}

Ground electronic state is ${ }^{2} \mathrm{~B}_{1}$.

[1] G. Duxbury, M. Horani and J. Rostas, Proc. Roy. Soc. (London) A331, 109 (1972).

\section{Hydrogen Disulfide (Disulfane)}

\begin{tabular}{ll|ll}
$\mathrm{H}_{2} \mathrm{~S}_{2}$ & \multicolumn{2}{c}{} & $\mathrm{C}_{2}$ \\
\hline \hline Bond & Effective & Angie & Effective \\
\hline & & & \\
$\mathrm{SS}$ & $2.058 \mathrm{~B}$ & HSS & $98.1 \mathrm{~B}$ \\
$\mathrm{SH}$ & $1.345 \mathrm{~B}$ & Dihedral & $90.8 \mathrm{~B}$ \\
\hline
\end{tabular}

Parameters were recalculated from reported data. The SD distance was assumed to be $0.003 \AA$ shorter than the SH distance.

[1] G. Winnewisser, J. Mol. Spectrosc. 41, 534. (1972).

[2] G. Winnewisser, M. Winnewisser, and W. Gardy, J. Chem. Phys. 49, 3465 (1968).

\section{Hydrogen Selenide}

$\mathrm{H}_{2} \mathrm{Se}$

\begin{tabular}{cccc}
\hline Bond & Effective & Average & Equilibrium \\
\hline HSe & $1.469 \mathrm{D}$ & $1.475 \mathrm{~A}$ & $1.460 \mathrm{~A}$ \\
\hline & Effective & Average & Equilibrium \\
\hline Angle & $90.9 \mathrm{D}$ & $90.6 \mathrm{~A}$ & $90.6 \mathrm{~A}$
\end{tabular}

The effective structure was calculated from the rotational cor stants of Ref. 1. The error is based on the spread in the dimensio calculated from the $I_{\mathrm{A}}, I_{\mathrm{B}} ; l_{\mathrm{B}}, I_{\mathrm{C}}$; or $I_{\mathrm{A}}, I_{\mathrm{C}}$ pairs. The average stru ture is that of Ref. 2. The equilibrium constants are those of Ref. I and, again the error is based on the spread in the dimensions of tained from the various pairs of parameters. Lines from Ref. 3 wer included with infraned data in Ref. 1 to ubtain the rolational con stants.

[1] R. A. Hill and T. H. Edwards, J. Chem. Phys. 42, 1391 (1965)

[2] T. Oka and Y. Morino, J. Mol. Spectrosc. 8, 300 (1962).

[3] A. W. Jache, P. W. Moser and W. Gordy, J. Chem. Phys. 25 209 (1956).

\section{Silylene}

\begin{tabular}{cc|cc}
$\mathrm{H}_{2} \mathrm{Si}$ & & & $\mathrm{C}_{2}$ \\
\hline Bond & Effective & Angle & Effective \\
\hline $\mathrm{SiH}$ & $1.516 \mathrm{C}$ & $\mathrm{HSiH}$ & $92.1 \mathrm{~B}$ \\
\hline
\end{tabular}

[1] I. Dubois, Can. I. Phys, 46, 2485 (1968).

\section{Hydrogen Telluride}

\begin{tabular}{lll|lll}
$\mathrm{H}_{2} \mathrm{Te}$ & & $\mathrm{C}_{2 v}$ \\
\hline Bond & Effective & Equilibrium & Angle & Effective & Equilibrium \\
\hline $\mathrm{HTe}$ & $1.653 \mathrm{D}$ & $1.658 \mathrm{~B}$ & $\mathrm{HTeH}$ & $90.3 \mathrm{D}$ & $90.2 \mathrm{~B}$ \\
\hline
\end{tabular}

[1] N. K. Moncur, P. D. Willson, and T. H. Edwards, J. MolSpectrosc. 52, 380 (1974). 
Hydrogen-Xenon(1/1

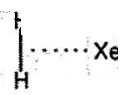

\begin{tabular}{lll}
$\mathrm{H}_{2} \mathrm{Xe}$ & Undefined \\
\hline Bonc & Effective \\
\hline $\begin{array}{l}\text { Mean value between center of } \\
\text { mass of } \mathrm{H}_{2} \text { and Xe atom }\end{array}$ & $4.25 \mathrm{X}$ \\
\hline
\end{tabular}

[1] A. R. W. McKellar and H. L. Welsh, J. Chem. Phys. 55, 595 (1971).

\section{lodosilane}

\begin{tabular}{lll|llll}
$\mathrm{H}_{3} \mathrm{ISi}$ & & \multicolumn{1}{c}{$\mathrm{ISiH}_{3}$} & & $\mathrm{C}_{3 \mathbf{v}}$ \\
\hline \hline & & & & & \\
Bond & Substitution & Effective & Angle & Substitution & Effective \\
& & & & & \\
\hline & & & & & & \\
$\mathrm{SiI}$ & $2.437 \mathrm{~A}$ & $2.437 \mathrm{~A}$ & HSiI & $107.8 \mathrm{~A}$ & $108.4 \mathrm{~B}$ \\
$\mathrm{SiH}$ & $1.485 \mathrm{~B}$ & $1.487 \mathrm{~B}$ & & & & \\
\hline
\end{tabular}

[1] R. Kewley, P. M. McKinney, and A. G. Robiette, J. Mol. Spectrose. 34, 390 (1970).

[2] A. H. Sharbaugh, G. A. Heath, L. F. Thomas, and J. Sheridan, Nature (London) 171, 87 (1953).

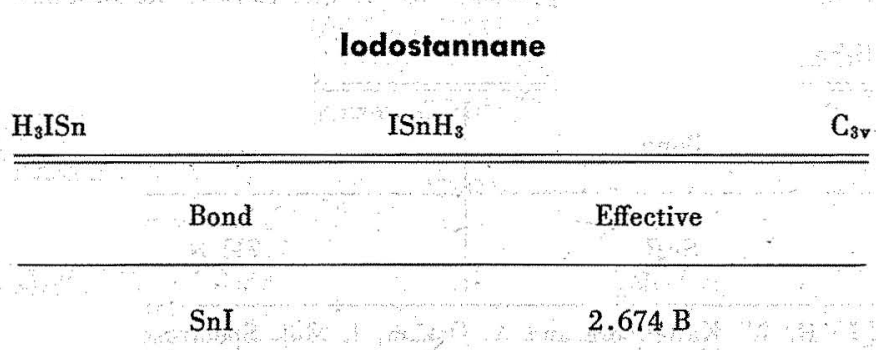

The projection of the $\mathrm{SnH}$ bond on the $\mathrm{SnI}$ axis was assumed.

[1] S. N. Wolf, L. C. Krisher and R. A. Gsell, J. Chem. Phys. 55, 2106 (1971).
Ammonis

\begin{tabular}{l|l|l|l|l}
\hline \hline Bond & Effective & Average & Substitution & Equilibriul \\
\hline NH & $1.017 \mathrm{~B}$ & $1.024 \mathrm{~B}$ & $1.014 \mathrm{D}$ & $1.012 \mathrm{~B}$ \\
\hline \hline Angle & Effective & Average & Substitution & Equilibriun \\
\hline HNH & $107.8 \mathrm{~B}$ & $107.3 \mathrm{~B}$ & $107.1 \mathrm{D}$ & $106.7 \mathrm{~B}$ \\
\hline
\end{tabular}

[1] W. S. Benedict and E. K. Plyler, Can. J. Phys. 35, 1235 (1957)

[2] P. Helminger, F. C. DeLucia and W. Gordy, J. Mol. Spectrose 39, 94 (1971).

[3] Y. Morino, K. Kuchitsu and S. Yamamoto, Spectrochim. Act: 24.A, 335 (1968).

\section{Hydroxylamine}

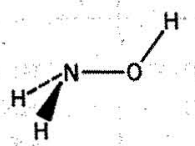

$\mathrm{H}_{3} \mathrm{NO}$

\begin{tabular}{ll|ll}
\hline Bond & Effective & Angle & Effective \\
& & & \\
$\mathrm{NH}$ & $1.016 \mathrm{C}$ & $\mathrm{HNH}$ & $107.1 \mathrm{D}$ \\
$\mathrm{NO}$ & $1.453 \mathrm{~B}$ & $\mathrm{HNO}$ & $103.2 \mathrm{C}$ \\
$\mathrm{OH}$ & $0.962 \mathrm{D}$ & $\mathrm{NOH}$ & $101.4 \mathrm{D}$ \\
\hline
\end{tabular}

[1] S. Tsunekawa, J. Phys. Soc. Japan, 33, 167 (1972).

\section{Phosphine}

\begin{tabular}{lll|lll}
$\mathrm{H}_{3} \mathrm{P}$ & & & & $\mathrm{PH}_{3 \mathrm{v}}$ \\
\hline \hline Bond & Effective & Average & Angle & Effective & Average \\
\hline & & & & & \\
\hline $\mathrm{PH}$ & $1.420 \mathrm{~A}$ & $1.427 \mathrm{~A}$ & $\mathrm{HPH}$ & $93.3 \mathrm{~A}$ & $93.3 \mathrm{~A}$ \\
\hline
\end{tabular}

[1] F. Y. Chu and T. Oka, J. Chem. Phys. 60, 4612 (1974).

[2] A. G. Maki, R. L. Sams and W. B. Olson, J. Chem. Phys. 58, 4502 (1973).

[3] P. Helminger and W. Gordy, Phys. Rev. 188, 100 (1969). 
Stibine

\begin{tabular}{|c|c|c|c|c|c|}
\hline $\mathrm{H}_{3} \mathrm{Sb}$ & \multicolumn{4}{|c|}{$\mathrm{SbH}_{3}$} & $\mathrm{C}_{3 \mathrm{v}}$ \\
\hline Bond & Substitution & Effective & Angle & Substitution & Effective \\
\hline $\mathrm{SbH}$ & $1.703 \mathrm{C}$ & $1.710 \mathrm{C}$ & HSbH & $91.5 \mathrm{C}$ & $91.7 \mathrm{C}$ \\
\hline
\end{tabular}

[1] P. Helminger, E. L. Beeson, Ir., and W. Gordy, Phys. Rev, A 3, 122(1971).

[2] A. W. Jache, G. S. Blevins, and W. Gordy, Phys. Rev. 97, 680 (1955).

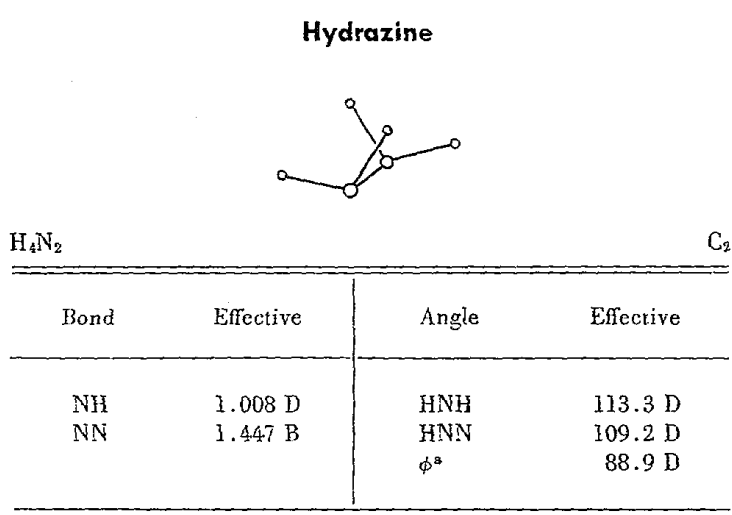

$\mathrm{NH}$ bonds and HNï bond angles have been assumed to be identical.

a Dihedral angle.

[I] S. Tsunekawa, J. Phys. Soc. Japan 41, 2077 (1976).

[2] T. Kasuya, Sci. Pap. Inst. Phys. Chem. Res. Tokyo 56, I (1962)

\section{Water dimer}

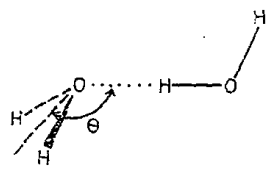

$\mathrm{H}_{4} \mathrm{O}_{2}$

$\mathrm{C}_{\mathrm{s}}$

Assuming unchanged monomer geometry, the $0 \cdots 0$ distance is reported as $2.98(\mathrm{X})$, and the angle $\theta$ is $58^{\circ}(\mathrm{X})$.

[1] T. R. Dyke, K, M. Mack and J. S. Muenter, J. Chem. Phys. 66, $498(1977)$.

\section{Biphosphine-4}

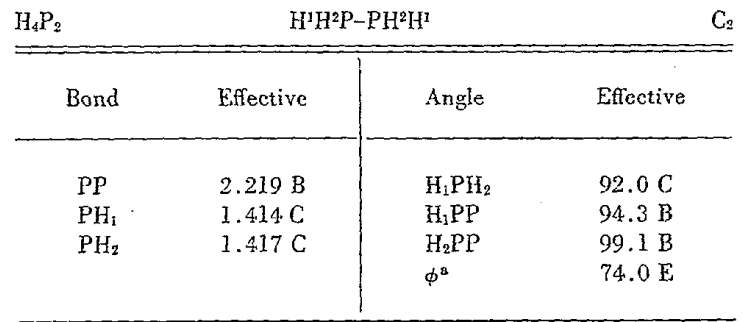

a Dihedral angle measured from cis position. Hydrogens num. bered $H_{2}$ are the nearly eclipsed pair.

[I] J. R. Durig, L. A. Carreira and J. D. Odom, J. Amer. Chem. Soc. 96, 2688 (1974).

\section{Silane}

\begin{tabular}{|c|c|c|}
\hline Bond & Effective & Substitution \\
\hline $\mathrm{SiH}$ & I. $481 \mathrm{~A}$ & $1.479 \mathrm{~A}$ \\
\hline
\end{tabular}

[1] M. Dang-Nhu, G. Pierre and R. Saint-Loup, Mol. Phys. 28, 447 (1974).

[2] R. W. Lovejoy and W. B. Olson, J. Chem. Phys. 57, 2224 (1972).

\section{Stannane}

$\begin{aligned} & \text { Bond } \mathrm{H}_{4} \mathrm{Sn} \\ & \text { SnH } \text { Effective } \\ & 1.711 \mathrm{~B}\end{aligned}$

[1] H. W. Kattenberg and A. Oskam, J. Mol. Spectrosc. 51, 377 (1974).

\section{Disilane}

$\mathrm{H}_{6} \mathrm{Si}_{2} \quad \mathrm{H}_{3} \mathrm{SiSiH}_{3} \quad \mathrm{D}_{3 \mathrm{~d}}$

\begin{tabular}{cc|cc} 
Bond & Effective & Angle & Effective \\
\hline SiSi & $2.327 \mathrm{C}$ & HSiH & $107.8 \mathrm{C}$ \\
\hline
\end{tabular}

SiH and SiD distances were assumed.

[I] K. C. Shotton, A. G. Lee and W. J. Jones, J. Raman. Spectrose. $I, 243(1973)$. 


\section{Nifrogen Dioxide}

$\mathrm{YO}_{2}$

\begin{tabular}{lll|lll} 
Hond Substitution Average & Angle & Substitution Average \\
NO & $1.197 \mathrm{~B}$ & $1.200 \mathrm{~B}$ & ONO & $133.8 \mathrm{~B}$ & $133.8 \mathrm{~B}$ \\
\hline
\end{tabular}

Ground state is ${ }^{2} A_{1}$.

Substitution values recomputed from reported rotational consiants.

[!] G. R. Bird, J. C. Baird, A. W. Jache, J. A. Hodgeson, R. F. Curl, Jr., A. C. Kunkle, J. W. Bransford, J. Rastrup-Andersen, and J. Rosenthal, J. Chem. Phys. 40, 3378 (1964).

[2] V. W. Laurie and D. R. Herschbach, J. Chem. Phys. 37, 1687 (1962)

[3] G. R. Bird, J. Chem. Phys, 25, 1040 (1956).

\section{Nitrous Oxide}

\begin{tabular}{c|c} 
Bond & $\mathrm{N}-\mathrm{N}-\mathrm{O}$ \\
\hline NN & Equilibrium \\
NO & $1.128 \mathrm{~A}$ \\
& $1.184 \mathrm{~A}$ \\
\hline
\end{tabular}

[1] K. Narahari Rao, Ann. New York Acad. Sci. 220, 17 (1973).

[2] C. A. Burrus and W. Gordy, Phys. Rev. 101, 599 (1956).

[3] C. H. Townes and A. L. Schawlow, Microwave Spectroscopy, McGraw-Hill, New York (1955).

\section{Dinitrogen Oxide $(+1)$ Ion}

\begin{tabular}{cl}
$\mathrm{N}_{2} \mathrm{O}^{+}$ & Effective \\
\hline Bond & \\
\hline $\mathrm{NN}$ & $1.155 \mathrm{C}$ \\
$\mathrm{NO}$ & $1.185 \mathrm{C}$
\end{tabular}

Ground electronic state is ${ }^{2} 11$.

[1] J. H. Callomon and F. Creutzberg, Phil. Trans. Roy. Soc. A277, 157 (1974).
Dinitrogen Irioxide

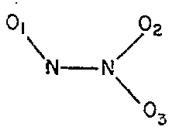

\begin{tabular}{|c|c|c|c|}
\hline Bond & Substitution & Angle & Substitution \\
\hline NN & $1.864 \mathrm{~B}$ & $\mathrm{NNO}_{1}$ & $105.1 \mathrm{~B}$ \\
\hline $\mathrm{NO}_{\mathrm{I}}$ & $1.142 \mathrm{C}$ & $\mathrm{NNO}_{2}$ & $112.7 \mathrm{~B}$ \\
\hline $\mathrm{NO}_{2}$ & $1.202 \mathrm{C}$ & $\mathrm{NNO}_{3}$ & $117.5 \mathrm{~B}$ \\
\hline $\mathrm{NO}_{3}$ & $1.217 \mathrm{~B}$ & & \\
\hline
\end{tabular}

11] A. H. Brittain, A. P. Cox and R. L. Kuczkowski, Trans. Faraday Soc. 65,1963 (1969).

\begin{tabular}{ll}
\multicolumn{1}{c}{ Azido } & \\
\hline Bond & Effective \\
\hline NN & $1.181 \mathrm{~A}$
\end{tabular}

Ground electronic state is ${ }^{2} \mathrm{~T}_{\mathrm{g}}$.

[1] A. E. Donglas and W. J. Jones, Can. J. Phys. 43, 2216 (1965).

\section{Disulfur monoxide}

\begin{tabular}{lll|llll} 
OS2 & \multicolumn{2}{c}{$\mathrm{S}_{2} \mathrm{O}$} & $\mathrm{C}_{\mathrm{B}}$ \\
\hline Bond & Substitution & Effective & Angle & Substitution Effective \\
\hline & & & & & \\
$\mathrm{SS}$ & $1.882 \mathrm{~B}$ & $1.885 \mathrm{~B}$ & $\mathrm{SSO}$ & $118.3 \mathrm{~B}$ & $118.1 \mathrm{~B}$ \\
$\mathrm{SO}$ & $1.464 \mathrm{~B}$ & $1.462 \mathrm{~B}$ & & &
\end{tabular}

[1] E. Tiemann, J. Hoeft, F. J. Lovas and D. R. Johnson, J. Chem. Phys. 60, 5000 (1974).

[2] D. J. Meschi and R. J. Myers, J. Mol. Spectrose. 3, 405 (1959). 


\section{Sulfur Dioxide}

\begin{tabular}{ccccc}
$\mathrm{O}_{3} \mathrm{~S}$ & $\mathrm{SO}_{2}$ & $\mathrm{C}_{2 v}$ \\
\hline Parameter & Equilibrium & Average & Substitution & Efective \\
\hline S0 & $1.431 \mathrm{~A}$ & $1.435 \mathrm{~A}$ & $1.433 \mathrm{~A}$ & $1.434 \mathrm{~A}$ \\
OSO & $119.3 \mathrm{~A}$ & $119.4 \mathrm{~A}$ & $119.6 \mathrm{~A}$ & $119.4 \mathrm{~A}$
\end{tabular}

[1] S. Saito, J. Mol. Spectrose. 30, I (1969).

[2] Y. Morino, Y. Kikuchi, S. Saito, and E. Hirota, J. Mol, Spectrose. 13, 95 (1964).

[3] R. van Riet and C. Stenbeckeiers, Mém. Acad. Roy. Belg. 36 , fasc. 8 (1965).

[4] M. H. Sirvetz, J. Chem. Phys. 19, 938 (1951).

[5] G. F. Crable and W. V. Smith, J. Chem. Phys. 19, 502 (1951).

[6] B. P. Dailey, S. Golden, and E. B. Wilson, Phys. Rev. 72, 87 l (1947).

\section{Sulfur monoxide dimer}

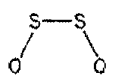

$0_{2} \mathrm{~S}_{2}$

\begin{tabular}{lll|lll}
\hline Bond & Substitution Effective & Angle & Suhstitution Effective \\
\hline SS & $2.025 \mathrm{~B}$ & $2.018 \mathrm{~B}$ & OSS & $112.7 \mathrm{~B}$ & $112.9 \mathrm{~B}$ \\
SO & $1.458 \mathrm{~B}$ & $1.469 \mathrm{~B}$ & & & \\
\hline
\end{tabular}

The effective parameters were calculated from reported data.

[1] F. J. Lovas, E. Tiemann and D. R. Johnson, J. Chem. Phys. $60,5005(1974)$.

\section{Selenium dioxide}

$\mathrm{O}_{2} \mathrm{Se}$

\begin{tabular}{|c|c|c|c|c|c|}
\hline Bond & Equilibrium & Effective & Angle & Equilibrium & Effective \\
\hline $\mathrm{SeO}$ & $1.608 \mathrm{~A}$ & $1.609 \mathrm{~A}$ & OSeO & $113.8 \mathrm{~A}$ & $114.0 \mathrm{~A}$ \\
\hline
\end{tabular}

[1] F. Wirata and Y Marino, J Mol. Speetrose. 34, 370 (1970).

\section{Oxone}

\begin{tabular}{lll|ccc}
$\mathrm{O}_{3}$ & & $\mathrm{C}_{2 v}$ \\
\hline Bond & Equilibrium & Average & Angle & Equilibrium Average \\
\hline 00 & $1.272 \mathrm{~A}$ & $1.279 \mathrm{~A}$ & 000 & $116.8 \mathrm{~A}$ & $116.8 \mathrm{~A}$ \\
\hline
\end{tabular}

[I T. Tanaka and Y. Morino, I. Wol. Spertrnes 33, 538 (1970)

[2] R. H. Hughes, J. Chem. Phys. 24, 131 (1956); J. Chem, Phys. 21, 959 (1953).

[3] R. Trambarulo, S. N. Ghosh, C. A. Burrus, Jr., and WV. D. Gordy, J. Chem. Phys. 21, 851 (1953).

J. Phys. Chem. Ref. Data, Vol. 8, No. 3, 1979
Sulfur Trioxide

\begin{tabular}{cc}
$\mathrm{O}_{3} \mathrm{~S}$ & $\mathrm{D}_{3 \mathrm{~K}}$ \\
\hline Bond & Effective \\
\hline SO & $1.420 \mathrm{~A}$ \\
\hline
\end{tabular}

[1] A. Kalder and A. G. Maki, J, Mol. Struct: 15, 123 (1973).

\section{$C_{1}$ Molecules}

\section{Cyanogen bromide}

\begin{tabular}{ccc}
$\mathrm{CBrN}$ & $\mathrm{BrCN}$ & $\mathrm{C}_{\text {cov }}$ \\
\hline \hline Bond & Substitution & Equilibrium \\
\hline $\mathrm{B} 1 \mathrm{C}$ & $1.709 \mathrm{~A}$ & $1.790 \mathrm{D}$ \\
$\mathrm{CN}$ & $1.158 \mathrm{~A}$ & $1.157 \mathrm{~B}$
\end{tabular}

[1] C. H. Townes, A. N. Holden and F. R. Merritt, Phys. Rev. $7 \lambda, 64(1947)$.

[2] S. J. T'etenbaum, Phys. Rev. 86, 440 (1952).

[3] C. A. Burrus and W. Gordy, Phys. Rev. 101, 599 (1956).

[4] A. G. Maki and C. T. Gott, J. Chem. Phys. 36, 2282 (1962).

\section{Carbonyl bromide}

\begin{tabular}{|c|c|c|c|}
\hline Bond & Effective & Angle & Effective \\
\hline $\begin{array}{l}\mathrm{CO} \\
\mathrm{CBr}\end{array}$ & $\begin{array}{l}1.172 \mathrm{C} \\
1.917 \mathrm{C}\end{array}$ & $\mathrm{BrCBr}$ & $112.3 \mathrm{C}$ \\
\hline
\end{tabular}

[1] J. H. Carpenter, J. G. Smith, I. Thompion and D. H. Whiffen, J. C. S. Faraday Trans. 1976, 384 (1976).

\section{Carbonyl Chloride Fluoride}

\begin{tabular}{cc|cc} 
CCIFo & ClFCO & $\mathrm{C}_{9}$ \\
\hline Bond & Effective & Angle & Effective \\
\hline $\mathrm{CF}$ & $1.303 \times$ & FCCI & $112.0 \times$ \\
$\mathrm{CO}$ & $1.162 \times$ & $\mathrm{CICO}$ & $117.5 \mathrm{X}$ \\
$\mathrm{CCl}$ & $1.751 \mathrm{X}$ & &
\end{tabular}

[1] A. Mirri, A. Guarneri, P. Favero, and G. Zuliani, Nuovo Cimento 25, 265 (1962). 
Chlorofluorothiocarbonyl

\begin{tabular}{llll} 
& & \\
& & & \\
CCIFS & & & \\
\hline \hline Bond & Average & Substitution & Effective \\
\hline CCl & $1.718 \mathrm{C}$ & $1.709 \mathrm{D}$ & $1.704 \mathrm{D}$ \\
$\mathrm{CF}$ & $1.336 \mathrm{C}$ & $1.347 \mathrm{D}$ & $1.337 \mathrm{D}$ \\
$\mathrm{CS}$ & $1.589 \mathrm{C}$ & $1.592 \mathrm{D}$ & $1.600 \mathrm{D}$ \\
\hline & & & \\
\hline & & & \\
\hline Angle & Average & Substitution & Effective \\
\hline FCS & $124.0 \mathrm{C}$ & $123.2 \mathrm{C}$ & $122.8 \mathrm{C}$ \\
$\mathrm{CICS}$ & $127.6 \mathrm{C}$ & $127.8 \mathrm{C}$ & $127.7 \mathrm{C}$ \\
\hline
\end{tabular}

Distances and angles were recalculated from reported rotationai constants.

[1] R. Hamm, H. J. Kohrmann, H. Günther and W. Zeil, Z. Naturforsch. 31a, 594. (1976)

[2] II. J. Kohnuanu and W. Zeil, Z. Nahurfursth. 30a, 185 (1975).

\section{Cyanogen Chloride}

\begin{tabular}{cccc} 
CCIN & \multicolumn{2}{c}{ CCN } & $\mathrm{C}_{\infty}$ \\
\hline Bond & Equilibrium & Average & Substitution \\
\hline CN & $1.160 \mathrm{~B}$ & $1.162 \mathrm{~B}$ & $1.159 \mathrm{~B}$ \\
$\mathrm{CCl}$ & $1.629 \mathrm{~B}$ & $1.631 \mathrm{~B}$ & $1.631 \mathrm{~B}$
\end{tabular}

[I] W. J. Lafferty, D. R. Lide, and R. A. Toth, J. Chem. Phys. 43, 2063 (1965).

[2] J. K. Tyler and J. Sheridan, Trans. Faraday Soc. 59, 2661 (1963).

[3] C. H. Townes, A. N. Holden, and F. R. Merritt, Phys. Rev. 74, ill3 (1948).

[4] A. G. Smith, H. Ring, W. V. Smith, and W. Gordy, Phys. Rev. $74,370(1948)$

[5] C. H. Townes, A. N. Holden, and F. R. Merritt, Phys. Rev, 71, 64 (1947).
Chlorine Isocyanate

\begin{tabular}{|c|c|c|c|}
\hline Bond & Suòstitution & Angle & Substitution \\
\hline $\mathrm{CIN}$ & $1.705 \mathrm{~B}$ & CINC & $118.8 \mathrm{C}$ \\
\hline $\mathrm{NC}$ & $1.226 \mathrm{~B}$ & $\mathrm{NCO}^{\mathrm{a}}$ & $170.9 \mathrm{C}$ \\
\hline $\mathrm{CO}$ & $1.162 \mathrm{~B}$ & & \\
\hline
\end{tabular}

a $\mathrm{Cl}$ and $\mathrm{O}$ are trans.

[1] W. H. Hocking and M. C. L. Gerry, J. Mol. Spectrosc. 42. 547 (1972).

[2] W. H. Hocking, M. L. Williams and M. C. L. Gerry, J. Mol. Spectrosc. 58, 250 (1975).

Dichlorodifluoromethane

\begin{tabular}{|c|c|c|c|c|c|}
\hline Bond & Substitution & Effective & Angle & Substitution & Effective \\
\hline $\mathrm{CCl}$ & $1.744 \mathrm{C}$ & & $\mathrm{CICCI}$ & $112.6 \mathrm{C}$ & \\
\hline $\mathrm{CF}$ & & $1.345 \mathrm{~B}$ & $\mathrm{FCF}$ & & 100.2 \\
\hline
\end{tabular}

[1] H. Takeo and C. Matsumura, Bull. Chem. Soc. Japan 50,636 (1977).

Carbonic Dichloride

(Carbonyl chiloride or phosgene)

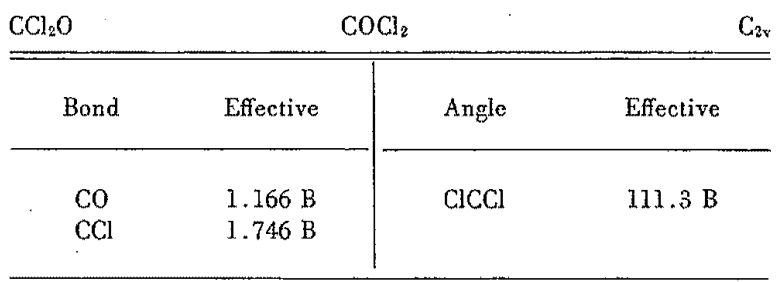

[1] G. Wilse Robinson, J. Chem. Phys, 21, 1741 (1953).

Trichlorofluoromethane

\begin{tabular}{cc|cc}
$\mathrm{CCl}_{3} \mathrm{~F}$ & & $\mathrm{C}_{3 \mathrm{~V}}$ \\
\hline \hline Bond & Effective & Angle & Effective \\
\hline $\mathrm{CCl}$ & $1.76 \mathrm{C}$ & $\mathrm{ClCCl}$ & $109.7 \mathrm{C}$ \\
$\mathrm{CF}$ & $1.33 \mathrm{D}$ & & \\
\hline
\end{tabular}

[1] M. W. Long, Q. Williams, and T. L. Weatherly, J. Chern. Phys 33, 508 (1960) 


\section{Cyanogen Fluoride}

\begin{tabular}{ccc}
\hline CFN & $\mathrm{ICN}$ & $\mathrm{C}_{\text {Sor }}$ \\
\hline Bond & Substitution & Efective \\
$\mathrm{CF}$ & & $1.262 \mathrm{~B}$
\end{tabular}

[1] J. K. Tyler and J. Sheridan, Trans. Faraday Soc. 89, 2663 (1963).

\section{Diffuoromethylene}

$\mathrm{CF}_{2}$

\begin{tabular}{lll|lll}
\hline \hline Bond Effective & Average & Angle & Effective & Average \\
\hline CF & $1.30 \mathrm{C}$ & $1.304 \mathrm{~A}$ & FCF & $104.9 \mathrm{C}$ & $104.8 \mathrm{~A}$
\end{tabular}

[1] W. H. Kirchhof, D. R. Lide, and F. X. Powell, J. Mol. Spec trosc, 47, 491 (1973)

[2] F. X. Powell and D. R. Lide, J. Chem. Phys. 45, 1067 (1966).

(Difluoromethylene)-amidiogen

\begin{tabular}{|c|c|c|c|}
\hline Bond & Effective & Angle & Effective \\
\hline $\mathrm{CN}$ & $1.265 \mathrm{D}$ & $\mathrm{FCF}$ & $113.5 \mathrm{C}$ \\
\hline
\end{tabular}

Ground electronic state is ${ }^{2} B_{2}$.

$\mathrm{CF}$ distance assumed $(1.310 \mathrm{i})$.

[1] R. N. Dixon, G. Duxbury, R. C. Mitchell and J. P. Simons, Proc. Roy. Soc. (London) A300, 405 (1967).

\section{Phosphorocyanidous Difluoride}

\begin{tabular}{|c|c|c|c|c|c|}
\hline Bond & Substitution & Effective & Angle & Substitution & Effective \\
\hline$p F$ & $1.566 \mathrm{C}$ & $1.567 \mathrm{C}$ & $F P F$ & $29.2 \mathrm{~B}$ & $29.1 \mathrm{~B}$ \\
\hline $\mathrm{PC}$ & $1.815 \mathrm{~B}$ & $1.811 \mathrm{~B}$ & FPC & $96.9 \mathrm{~B}$ & $97.1 \mathrm{~B}$ \\
\hline CN & $1.157 \mathrm{~B}$ & $2.158 \mathrm{~B}$ & $\mathrm{PCN}$ & $171.2 \mathrm{D}$ & $171.5 \mathrm{C}$ \\
\hline
\end{tabular}

[I] P. L. Lee, K. Cohn, and R. H. Schwendeman, Inorg. Chem. 11, 1917 (1972).
Difluerocyonamide

\begin{tabular}{|c|c|c|c|c|c|}
\hline $\mathrm{CH}_{2} \mathrm{~N}_{2}$ & & $\mathrm{NH}$ & & & $\mathrm{L}_{\varepsilon}$ \\
\hline Bond & Effective & Substitution & Angle & Effective & Substitution: \\
\hline $\mathrm{CN}$ & $1.151 \mathrm{~B}$ & $1.158 \mathrm{~B}$ & NCNa. & $169.7 \mathrm{E}$ & $173.9 \mathrm{E}$ \\
\hline $\mathrm{NC}$ & $1.392 \mathrm{C}$ & $1.386 \mathrm{C}$ & CNF & $104.7 \mathrm{D}$ & $105.4 \mathrm{bD}$ \\
\hline $\mathrm{NF}$ & $1.398 \mathrm{~B}$ & $1.399 \mathrm{~b} \mathrm{C}$ & FNF & $102.8 \mathrm{C}$ & $102.8^{\mathrm{bD}}$ \\
\hline
\end{tabular}

The cyanide is tilted away from the $\mathrm{NF}_{2}$ moiety.

${ }^{b} A$ mixed $r_{m}, r_{\mathrm{s}}$ parameter.

[1] P. I. Iee, K. Cohn and R. H. Schwendeman, Inorg. Chem. 11, 1921 (1972).

\begin{tabular}{|c|c|c|c|}
\hline \multirow{3}{*}{$=$} & \multicolumn{2}{|c|}{$\begin{array}{l}\text { Carbonic difluoride } \\
\text { (Carbonyl fluoride) }\end{array}$} & \\
\hline & \multicolumn{2}{|c|}{$\mathrm{COF}_{2}$} & \\
\hline & Effective & Substitution ${ }^{2}$ & Average \\
\hline $\begin{array}{l}\mathrm{CF} \\
\mathrm{CO}\end{array}$ & $\begin{array}{l}1.315 \mathrm{D} \\
1.170 \mathrm{E}\end{array}$ & $\begin{array}{l}1.312^{\circ} \mathrm{C} \\
1.125 \mathrm{C}\end{array}$ & $\begin{array}{l}1.317 \mathrm{~A} \\
1.170 \mathrm{~B}\end{array}$ \\
\hline Angle & Efrective & Substitution ${ }^{\mathrm{a}}$ & Average \\
\hline$F C F$ & $107.6 \mathrm{E}$ & $108.0^{\mathrm{E}} \mathrm{C}$ & $107.6 \mathrm{~B}$ \\
\hline
\end{tabular}

" Bond shortening correction to moments was applied prior to Kraitchman calculation.

A mixed $r_{s,} r_{s}$ parameter.

[1] D. F. Smith, M. Tidwell, D. V. P. Williams and S. J. Senatore, Phys. Rev. 83, 485 (1051).

[2] V. W. Laurie, D. T. Pence and R. H. Jackson, J. Chem. Phys. 37, 2995 (1962).

[3] T. Oka and Y. Morino, J. Mol. Spectrose. 11, 349 (1963).

[4] A. M. Mirri, F. Scappini, L. Innamorati and P. Favero, Spectrochim. Acta 25A, 1631 (1969).

[5] J. ł. Carpenter, J. Mol. Spectrosc. 50, 182 (1974).

Carbonothiaic Difuoride

(Thiocarbonyl Fiuoride)

\begin{tabular}{cc|cc}
$\mathrm{CF}_{2} \mathrm{~S}$ & \multicolumn{2}{c}{$\mathrm{SCF}_{2}$} & $\mathrm{C}_{25}$ \\
\hline Bond & Effective & Angle & Effective \\
\hline $\mathrm{CF}$ & $\begin{array}{l}1.315 \mathrm{C} \\
\mathrm{CS}\end{array}$ & FCF & $107.1 \mathrm{D}$ \\
\hline
\end{tabular}

[1] A. I. Careless, H. W. Kroto and B. M. Landsberg, Chem. Phys. 1., 371 (1973). 
Chloromethylene

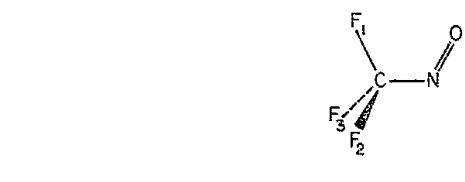

\begin{tabular}{ll|ll} 
Bond & Effective & Angle & Effective \\
\hline & & & \\
$\mathrm{CF}$ & $1.324 \mathrm{C}$ & $\mathrm{F}_{8} \mathrm{CN}$ & $113.9 \mathrm{D}$ \\
$\mathrm{NO}$ & $1.198 \mathrm{C}$ & $\mathrm{F}_{2} \mathrm{CN}_{1}$ & $107.5 \mathrm{C}$ \\
$\mathrm{CN}$ & $1.512 \mathrm{D}$ & $\mathrm{F}_{2} \mathrm{CF}_{3}$ & $109.8 \mathrm{C}$ \\
& & $\mathrm{CNO}^{2}$ & $112.4 \mathrm{C}$
\end{tabular}

CF bond distances assumed equal. $C F_{y}$ group is tilted toward wygen atom by $4.3^{\circ}$.

1 I] P. H. Turner and A. P. Cox, Chem. Phys. Lett. 39, 585 (1976).

\section{Terreftuoro(trifluoromethyl)phosphorane}

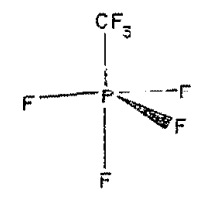

CIP

CF shown to be axially substituted

[1] E. A. Cohen and C. D. Cormwell, Inorg. Chem. 7, 398 (1968).

Tribromomethane

\begin{tabular}{ll|ll}
$\mathrm{CHB}_{3}$ & \multicolumn{2}{c}{$\mathrm{HCBr}_{3}$} & $\mathrm{C}_{3 \mathrm{~V}}$ \\
\hline Bond & Effective & Angle & Effective \\
& & & \\
$\mathrm{CH}$ & $1.068 \mathrm{D}$ & $\mathrm{BrCB}-$ & $110.8^{\circ} \mathrm{C}$ \\
$\mathrm{CBr}$ & $1.930 \mathrm{~B}$ & &
\end{tabular}

[1] A. Willians, J. T. Cox and W. Gordy, J. Chem. Phys. 20, 1524. (1952).

\begin{tabular}{|c|c|c|c|}
\hline $\mathrm{CHCl}$ & & & \\
\hline Bond & Effective & Angle & Effective \\
\hline $\mathrm{CH}$ & $1.12 \mathrm{G}$ & $\mathrm{HCCl}$ & $103.4 \mathrm{~B}$ \\
\hline $\mathrm{CCl}$ & $1.689 \mathrm{~B}$ & & \\
\hline
\end{tabular}

[1] A. J. Merer and D. N. Travis, Can. J. Phys, 44, 525 (1966).

\section{Chlorodifluoromethame}

\begin{tabular}{ccc|ll}
$\mathrm{CHClF}_{2}$ & & \multicolumn{2}{c}{$\mathrm{C}_{8}$} \\
\hline Bond & Substitution & Effective & Angle & Effective \\
& & & & \\
$\mathrm{CCl}$ & $1.747 \mathrm{D}$ & & $\mathrm{FCCl}$ & $110.1 \mathrm{D}$ \\
$\mathrm{CF}$ & & $1.350 \mathrm{D}$ & $\mathrm{FCF}$ & $107.0 \mathrm{D}$
\end{tabular}

$\mathrm{CH}$ bond and HCCl angle assumed.

[1] E. L. Beeson, T. L. Weatherly, and Q. Williams, J. Chem. Phys. 37, 2926 (1962).

[2] D. B. Mclay and C. K. Mann, Can. J. Phys. 40, 61 (1962).

\section{Formyl Chloride}

\begin{tabular}{cc|cc} 
CHClO & \multicolumn{1}{c}{ HClCO } & $\mathrm{C}_{\mathrm{g}}$ \\
\hline Bond & Substitution & Angle & Substitution \\
\hline & & & \\
$\mathrm{CH}$ & $1.096 \mathrm{~B}$ & $\mathrm{HCCl}$ & $109.9 \mathrm{D}$ \\
$\mathrm{CO}$ & $1.188 \mathrm{~B}$ & $\mathrm{HCO}$ & $126.5 \mathrm{C}$ \\
$\mathrm{CCl}$ & $1.760 \mathrm{C}$ & & \\
\hline
\end{tabular}

[1] H. Takeo and C. Matsumura, J. Chem. Phys. 64, 4536 (1976).

\section{Dichlorofivoromethane}

\begin{tabular}{cc|cc} 
CHCly & & $\mathrm{C}_{3}$ \\
\hline Bond & Effective & Angle & Effective \\
\hline $\mathrm{CCl}$ & $1.758 \mathrm{D}$ & $\mathrm{ClCCl}$ & $111.4 \mathrm{D}$ \\
$\mathrm{CF}$ & $1.346 \mathrm{D}$ & $\mathrm{ClCF}$ & $109.5 \mathrm{D}$
\end{tabular}

The parameters were calculated from the reported rotational constants. The $\mathrm{CH}$ distance was assumed to be $1.10 \mathrm{~A}$, and the CICH angle was assumed equal to the FCH angle.

[1] D. B. MeLay, Can. J. Phys. 42,720 (1964). 
Chloroform

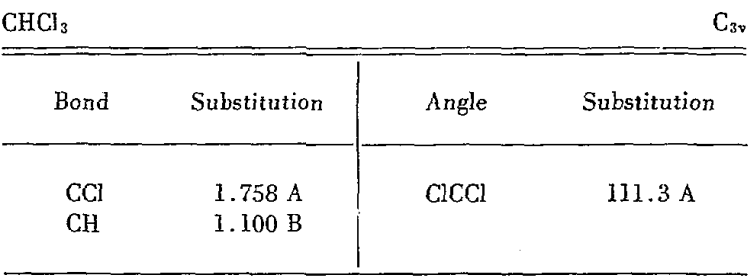

[1] M. Jen and D. R. Lide, J. Chem. Phys. 36, 2525 (1962).

\section{Fluoromethylene}

Bond
Effective

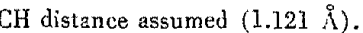

[1] A. J. Merer and D. X. Travis, Can. J. Phys. 44, 1541 (1966).

\section{Formyl Fluoride}

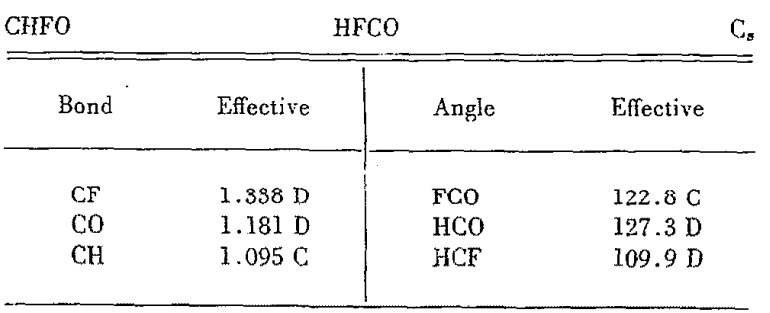

[1] K. F. Miller and K. F. Curl, Jr., J. Chem. Phys. 34, 1847 (1961). [2] O. H. LeBlanc, Jr., V. W. Laurie, and W. D. Gwinn, J. Chem. Phys. 33, 598 (1960).

\section{Trifluoromethane}

\begin{tabular}{cc|cc}
$\mathrm{CHF}_{3}$ & Engle & Eflective \\
\hline Bond & Efective & Angle \\
\hline $\mathrm{CH}$ & $\begin{array}{c}1.008 \mathrm{D} \\
\mathrm{CF}\end{array}$ & FCF & $108.8^{\circ} \mathrm{C}$ \\
\hline
\end{tabular}

[1] S. N. Ghosh, R. Trambarulo and W. Gordy. J. Chem. Phys. 20, 605 (1952).

\section{Hydrocyanic Acid}

\begin{tabular}{cccc} 
SHN & \multicolumn{1}{c}{ HCN } & Effective & Equilibrium \\
Bond & Substitution & C & \\
& & & \\
$\mathrm{CH}$ & $1.063 \mathrm{~B}$ & $1.064 \mathrm{D}$ & $1.065 \mathrm{~A}$ \\
$\mathrm{CN}$ & $1.155 \mathrm{~B}$ & $1.156 \mathrm{D}$ & $1.153 \mathrm{~A}$
\end{tabular}

[1] E. F. Pearson, R. A. Creswell, M. Winnewisser and G. Winnewisser, Z. Naturforsch. 31a, 1394 (1976).

[2] G. Winnewisser, A. G. Maki and D. R. Johnson, J. Mol. Spectrose. $39,149(19 / 1)$.

[3] F. de Lucia and W. Gordy, Phys. Rev. 187, 58 (1969).

[4] A. G. Maki, W. B. Olson and R. L. Sams, J. Mol. Spectrosc. 36, 433 (1970)

[5] T. Nakagawa and Y. Morino, J. Mol. Spectrosc. 31, 208 (1969).

[6] A. G. Maki, E. K. Plyler and R. Thibault, J. Opt. Soc. Am. 54, 869 (1964).

[7] H. C. Allen, E. D. Tidwell and E. K. Plyler, J. Chem. Phys. 25, 302 (1956).

[8] D. H. Rank, G. Skorinko, D. P. Eastman and T. A. Wiggins, J. Opt. Soc. Am. 50, 421 (1960).

[9] D. H. Rank, D. P. Eastman, B. S. Rao and T. A. Wiggins, J. Opt. Soc. Am. 51, 929 (1961).

[10] W. W. Brim, J. M. Hoffman, H. H. Nielsen and K. N. Rao, J. Opt. Soc, Am. 50, 1208 (1960).

[11] D. H. Rank, R. P. Ruth and K. L. Van der Sluis, J. Opt. Soc. Am. 42, 693 (1952).

[12] D. H. Rank, T. A. Wiggins, A. H. Guenther and J. N. Shearer, J. Opt. Soc. Am. 46, 953 (1956).

[13] D. H. Rank, A. H. Guenther, J. N. Shearer and T. A. Wiggins, J. Opt. Soc. Am. 47, 14.8 (1957).

\section{Isocyanic Acid}

\begin{tabular}{lll} 
CHN & HNC & Effective \\
\hline Bond & Substitution & $0.986 \mathrm{C}$ \\
$\mathrm{NH}$ & $0.986 \mathrm{~B}$ & $0.173 \mathrm{C}$ \\
$\mathrm{CN}$ & $1.172 \mathrm{~B}$ & 1.90 \\
\hline
\end{tabular}

[] E. F. Pearson, R. A, Creswell, M. Winnewisser and G. Winnewisser, Z. Naturforsch. 31a, 1394 (1976).

[2] R. A. Creswell, E. F. Pearson, M. Winnewisser and G. Winnewisser, Z. Naturforsch. 31a, 222 (1976).

[3] G. L. Blackman, R. D. Brown, P. D. Godirey and H. I. Gunn, Nature 261, 395 (1976) 
Fulminic Acid

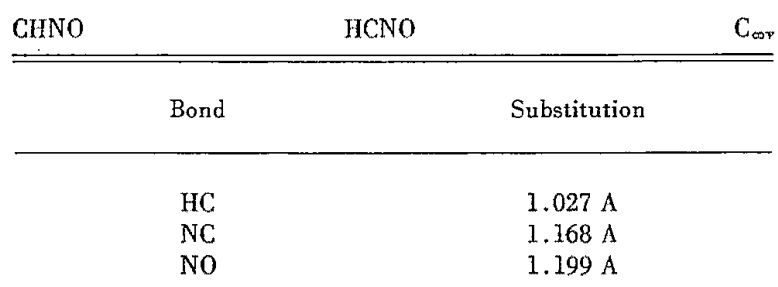

A linear model is assumed in arriving at the substitution structure. The molecule is actually quasilinear with a large amplitude bending vibration and an equilibrium $\mathrm{HCN}$ angle between $155^{\circ}$ and $170^{\circ}$.

[1] H. K. Bodenseh and M. F. Winnewisser, Z. Naturforsch. 24a, 1973 (1969).

[2] B. P. Winnewisser, M. F. Winnewisser, F. Winther, J. Mol. Spectrosc. 51, 65 (1974).

\section{Isocyanic acid}

\begin{tabular}{cc|cc} 
CHNO & \multicolumn{2}{c}{ HNCO } & $\mathrm{C}_{\mathrm{B}}$ \\
\hline \hline Bond & Substitution & Angle & Substitution \\
\hline $\mathrm{NC}$ & $1.209 \mathrm{~A}$ & HNC & $128.0 \mathrm{C}$ \\
$\mathrm{CO}$ & $1.166 \mathrm{~A}$ & & \\
$\mathrm{NH}$ & $0.986 \mathrm{D}$ & & \\
\hline
\end{tabular}

It was necessary to assume linearity of the $\mathrm{NCO}$ group. The reported values of $\mathrm{NII}$ and $\mathrm{HNC}$ are most strongly affected by this assumption, but the heavy-atom distances are also affected to a smaller extent.

[1] W. H. Hocking, M. C. L. Gerry and G. Winnewisser, Can. J. Phys. 53, 1869 (1975).

[2] W. H. Hocking. M. C. I. Gerry and C. Winnewiseer, Astrnphys J. 174, L93 (1972).

\section{Isothiocyanic Acid}

\begin{tabular}{cc|cc} 
CHNS & \multicolumn{2}{c}{ HNCS } & $\mathrm{C}_{\mathrm{B}}$ \\
\hline \hline Bond & Effective & Angle & Effective \\
\hline CS & $1.561 \mathrm{X}$ & HNC & $135.0 \mathrm{X}$ \\
$\mathrm{NC}$ & $1.216 \mathrm{X}$ & & \\
$\mathrm{NH}$ & $0.989 \mathrm{X}$ & & \\
\hline
\end{tabular}

The NCS angle has been assumed to be $180^{\circ}$.

[I] R. Kewley, K. V. L. N. Sastry, and M. Winnewisser, J. MoI Spectrosc. 10, 418 (1963).

[2] C. I. Beard and B. P. Dailey, J. Chem. Phys. 18, 1437 (1950); 19, 975 (1951)
Cyanoamidogen

\begin{tabular}{cc|cc} 
CHN $_{z}$ & \multicolumn{2}{c}{ HNCN } & $\mathrm{C}_{s}$ \\
\hline \hline Bond & Effective & Angle & Effective \\
\hline $\mathrm{NH}$ & $\begin{array}{l}1.034 \mathrm{D} \\
2.470 \mathrm{~A}\end{array}$ & $\mathrm{HNC}$ & $116.5 \mathrm{E}$ \\
$\mathrm{N} \cdots \mathrm{N}$ & & \\
\hline
\end{tabular}

NCN angle assumed $\left(180^{\circ}\right)$

Ground electronic state is ${ }^{2} A$ ".

[1] G. Heraberg and P. A. Warsop, Can. J. Phys. 41, 286 (1963).

\begin{tabular}{cc|cc}
\multicolumn{4}{c}{ Formyl } \\
CHO & HCO & Angle & Effective \\
\hline \hline Bond & Effective & nCo & $124.9 \mathrm{D}$ \\
\hline $\begin{array}{cc}\mathrm{ClI} \\
\mathrm{CO}\end{array}$ & $\begin{array}{l}1.225 \mathrm{C} \\
1.175 \mathrm{C}\end{array}$ & & \\
\hline
\end{tabular}

Ground state is ${ }^{2} A^{\prime}$.

[1] J. M. Brown and D. A. Kamsay, Can. J. Phys. 53, 2232 (19/5).

[2] J. A. Austin, D. H. Levy, C. A. Gottlieb and H. E. Radford, J. Chem. Phys. 60, 207 (1974).

[3] S. Saito, Astrophys. J. 178, 95 (1972).

\section{Methylidene Phosphine}

\begin{tabular}{lcc} 
CHP & HCP & $\mathrm{C}_{\text {कv }}$ \\
\hline Bond & Effective & Substitution \\
\hline $\mathrm{HC}$ & & $1.068 \mathrm{~A}$ \\
$\mathrm{CP}$ & $1.542 \mathrm{~A}$ &
\end{tabular}

[1] J. K. Tyler, J. Chem. Phys. 40, 1170 (1964).

\section{Methylene}

\begin{tabular}{cc|cc}
$\mathrm{CH}_{2}$ & & $\mathrm{C}_{2 \mathrm{~V}}$ \\
\hline \hline Bond & Effective & Angle & Effective \\
\hline $\mathrm{CH}$ & $1.078 \mathrm{C}$ & $\mathrm{HCH}$ & $136 \mathrm{E}$ \\
\hline
\end{tabular}

Ground electronic state is ${ }^{3} B_{1}$.

[1] G. Herzberg and J. W. C. Johns, J. Chem. Phys. 54, 2276 (1971). 
Dibromomethane

\begin{tabular}{cl|ll}
\multicolumn{2}{c}{} & & $\mathrm{C}_{28} \mathrm{Br}_{2}$ \\
\hline \hline Bond & Effective & Angle & Effective \\
\hline & & & \\
$\mathrm{CBr}$ & $1.927 \mathrm{C}$ & $\mathrm{BrCBr}$ & $112.7 \mathrm{~B}$ \\
$\mathrm{CH}$ & $1.08 \mathrm{X}$ & $\mathrm{HCH}$ & $114 \mathrm{X}$
\end{tabular}

[1] D. Chadwick and D. J. Millen, Trans. Faraday Soc. 67, 1539 (1971).

\section{Chlorofluoromethane}

\begin{tabular}{cc|cc}
\multicolumn{1}{c|}{$\mathrm{CH}_{2} \mathrm{CIF}$} & & $\mathrm{C}_{8}$ \\
\hline Bond & Effective & Argle & Effective \\
\hline $\mathrm{CH}$ & $1.095^{*}$ & $\mathrm{FCCl}$ & $109.2 \mathrm{X}$ \\
$\mathrm{CF}$ & $1.333^{\mathrm{X}}$ & $\mathrm{HCCl}$ & $109.9 \mathrm{X}$ \\
$\mathrm{CCl}$ & $1.797 \mathrm{X}$ & $\mathrm{HCF}$ & $109.2 \mathrm{X}$ \\
& & $\mathrm{HCH}$ & $109.4 \mathrm{X}$ \\
\hline
\end{tabular}

a This value was ansumed.

[I] N. Muller, J. Am. Chem. Soc. 75, 860 (1953).

[2] R. N. Nandi and A. Chatteriji, Spectrochim. Acta 3IA, 603 (1975).

\section{Dichloromethane}

$\mathrm{CH}_{2} \mathrm{Cl}_{2}$

\begin{tabular}{ll|ll}
\hline Bond & Effective & Angle & Effective \\
\cline { 3 - 4 } CII & $1.068 \mathrm{D}$ & HCH & $112.0 \mathrm{E}$ \\
$\mathrm{CCl}$ & $1.772 \mathrm{~B}$ & $\mathrm{CICCl}$ & $111.8 \mathrm{C}$ \\
\hline
\end{tabular}

[1] R. J. Meyers and W. D. Gwinn, J. Chem. Phys. 20, 1420 (1952).

\section{Hydrogen cyanide-hydrogen \\ fluoride dimer}

$\mathrm{CH}_{2} \mathrm{EN} \quad \mathrm{HCN} \cdots \mathrm{HF}$

If monomer moieties are chosen as in the free monomers, $N F=$ $2.796(\mathrm{X})$.

[1] A. C. Legon, D. J. Millen and S. C. Rogers, Chem. Phys. Lett. 41, $137(1076)$. $2 \mathrm{v}$
Difluoromethane

\begin{tabular}{|c|c|c|c|c|c|}
\hline \multicolumn{3}{|c|}{$\mathrm{CH}_{2} \mathrm{~F}_{2}$} & \multirow[b]{2}{*}{ in } & \multicolumn{2}{|c|}{$\mathrm{C}_{2}$} \\
\hline Bond & Substitution & Effective & & Sublulution & Effective \\
\hline $\begin{array}{l}\mathrm{CF} \\
\mathrm{CH}\end{array}$ & $1.093 \mathrm{~B}$ & $1.357 \mathrm{~B}$ & $\begin{array}{l}\text { Fel: } \\
\text { IIS:Il }\end{array}$ & $11 \therefore .7 \mathrm{~B}$ & $108.3 \mathrm{~B}$ \\
\hline
\end{tabular}

[1] E. Hirota and T. Tanaka, J. Mol. Simwirme, 34, 222 (1970).

[2] D. R. Lide, Jr., J. Amer. Chem. Sor. 7.4, 35.4, (19)32).

(Trifluoromethyl)phosphise

\begin{tabular}{|c|c|c|c|}
\hline \multicolumn{4}{|l|}{$\mathrm{CH}_{2} \mathrm{~F}_{3} \mathrm{P}$} \\
\hline Bond & Effective & Angle & Effective \\
\hline $\mathrm{CP}$ & $1.90 \times$ & FCF & $108 X$ \\
\hline \multirow[t]{2}{*}{$\mathrm{PH}$} & $1.43 x$ & $\mathrm{CPH}$ & $92 X$ \\
\hline & & HPH & $97 \times$ \\
\hline
\end{tabular}

CF bond length was assumed.

[1] I. Yang, C. Britt, A. Cowley, and J. Boggs, J. Chem. Phys., 48, 812 (1968).

\begin{tabular}{|c|c|c|c|c|}
\hline \multicolumn{5}{|c|}{ Diazomethane } \\
\hline $\mathrm{CH}_{2} \mathrm{~N}_{2}$ & \multicolumn{3}{|c|}{$\mathrm{H}_{2} \mathrm{C}=\mathrm{N}=\mathrm{N}$} & $C_{2 v}$ \\
\hline Bond & Substitution & Effective & Angle & Effective \\
\hline NN & $1.140 \mathrm{~B}$ & & $\mathrm{HCH}$ & $126.0 \mathrm{C}$ \\
\hline $\mathrm{CN}$ & $1.300 \mathrm{~B}$ & & & \\
\hline $\mathrm{CH}$ & & $1.075 \mathrm{C}$ & & \\
\hline
\end{tabular}

[1] A. P. Cox, L. F. Thomas and J. Sheridan, Nature 181, 1000 (1958).

[2] J. Sheridan, Advances in Molecular Spectroscopy (Pergamon Press, Ltd., London, 1062) p. 139.

[3] C. B. Moore, J. Chem. Phys, 39, 1884 (1963).

\section{Formaldehyde}

\begin{tabular}{lll|lll}
$\mathrm{CH}_{2} \mathrm{O}$ & $\mathrm{H} \mathrm{CO}$ & $\mathrm{C}_{2 \mathrm{~V}}$ \\
\hline Bond & Substitution & Average & Angle & Substitution & Average \\
\hline $\mathrm{CO}$ & $1.206 \mathrm{~B}$ & $1.208 \mathrm{~B}$ & $\mathrm{HCH}$ & $116.6 \mathrm{~B}$ & $116.5 \mathrm{C}$ \\
$\mathrm{CH}$ & $1.108 \mathrm{~B}$ & $1.116 \mathrm{C}$ & & & \\
\hline
\end{tabular}

[1] k. Tikiugi and T. Oka, J. Phye. Soc. Japan 18, 1174. (1963). [2] T. Oka, J. Phys. Soc. Japan 15, 2274 (1960). 
Methanethial S-oxide

(Sulfine)

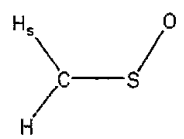

\begin{tabular}{cc|cc}
\multicolumn{1}{c|}{} & & & $\mathrm{C}_{3}$ \\
\hline \hline Bond & Substitution & Angle & Substitution \\
\hline & & & \\
$\mathrm{CH}_{8}$ & $1.085 \mathrm{C}$ & $\mathrm{HCH}$ & $121.9 \mathrm{C}$ \\
$\mathrm{CH}$ & $1.077 \mathrm{C}$ & $\mathrm{H}_{8} \mathrm{CS}$ & $122.5 \mathrm{~B}$ \\
$\mathrm{CS}$ & $1.610 \mathrm{~B}$ & $\mathrm{HCS}$ & $115.6 \mathrm{C}$ \\
$\mathrm{SO}$ & $1.469 \mathrm{~B}$ & $\mathrm{CSO}$ & $114.7 \mathrm{~B}$ \\
\hline
\end{tabular}

[1] R. E. Penn and R. J. Olsen, J. Mol. Spectrosc. 61, 21 (1976).

\section{Formic Acid}

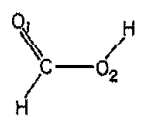

$\mathrm{CH}_{2} \mathrm{O}_{3}$

\begin{tabular}{cc|cc}
\hline \hline Bond & Effective & Angle & Effective \\
\hline & & & \\
$\mathrm{CH}$ & $1.097 \mathrm{X}$ & $\mathrm{OCO}$ & $124.6 \mathrm{X}$ \\
$\mathrm{CO}$ & $1.202 \times$ & $\mathrm{HCO}$ & $124.1 \mathrm{X}$ \\
$\mathrm{CO}$ & $1.343 \mathrm{X}$ & $\mathrm{COH}$ & $106.3 \mathrm{X}$ \\
$\mathrm{OH}$ & $0.972 \mathrm{X}$ & & \\
\hline
\end{tabular}

Numerous small coordinateo lead to structuro of un cortain quality. [1] G. Kwei and R. Curl, J. Chem. Phys. 32, 1592 (1960).

[2] A. M. Mirri, Nuovo Cimento 18, 849 (1960).

[3] R. Lerner, J. Friend, and B. Dailey, J. Chem. Phys. 23, 210 (1955).

\section{Thioformaldehyde}

\begin{tabular}{cccc}
$\mathrm{CH}_{2} \mathrm{~S}$ & \multicolumn{2}{c}{$\mathrm{H}_{2} \mathrm{CS}$} & $\mathrm{C}_{2 \mathrm{v}}$ \\
\hline \hline Bond & Substitution & Angle & Substitution \\
\hline $\mathrm{CH}$ & $1.093 \mathrm{C}$ & $\mathrm{HCH}$ & $116.9 \mathrm{C}$ \\
$\mathrm{CS}$ & $1.611 \mathrm{~B}$ & &
\end{tabular}

[1] D. R. Johnson, F. X. Powell, and W. H. Kirchhoff, J. Mol. Spectrose. 39, 136 (1971).
Methyl

$\mathrm{CH}_{3}$ $D_{3 \mathrm{~h}}$

\begin{tabular}{cc}
\hline Bond & Effective \\
\hline $\mathrm{CH}$ & $1.079 \mathrm{~B}$
\end{tabular}

Ground electronic state is ${ }^{2} A_{2}^{\prime \prime}$.

The bond length is derived from $B_{0}$ of $\mathrm{CD}_{2}$. [1] G. Herzberg, Proc. Roy. Soc. A262, 291 (1961).

\section{Carbon Monoxide-Borane} (Borane carbonyl)

\begin{tabular}{|c|c|c|c|c|c|}
\hline $\mathrm{CH}_{3} \mathrm{BO}$ & & $\mathrm{H}_{3} \mathrm{~B}$ & & & $\mathrm{C}_{3 \mathrm{v}}$ \\
\hline Bond & $\begin{array}{c}\text { Substitu- } \\
\text { tion }\end{array}$ & Effective & Angle & $\begin{array}{c}\text { Substitu- } \\
\text { tion }\end{array}$ & Effective \\
\hline $\mathrm{BC}$ & $1.534 \mathrm{C}$ & $1.539 \mathrm{C}$ & $\mathrm{HBC}$ & $103.8 \mathrm{~A}$ & $103.6 \mathrm{~A}$ \\
\hline Co & $1.135 \mathrm{C}$ & $1.132 \mathrm{C}$ & HBII & $114.5 \mathrm{~B}$ & $114.6 \mathrm{~B}$ \\
\hline$B \cdots O$ & $2.669 \mathrm{~A}$ & $2.671 \mathrm{~B}$ & & & \\
\hline BHI & $1.222 \mathrm{~B}$ & $1.225 \mathrm{~B}$ & & & \\
\hline
\end{tabular}

Bromomethane

$\mathrm{CH}_{3} \mathrm{Br}$ $\mathrm{C}_{3 \mathrm{v}}$

\begin{tabular}{lcc|cc}
\hline \hline Bond & Substitution & Equilibrium & Angle & Equilibrium \\
\hline $\mathrm{CB}$ & $1.930 \mathrm{C}$ & $\begin{array}{l}1.933 \mathrm{C} \\
1.086 \mathrm{C}\end{array}$ & HCH & $111.2 \mathrm{C}$ \\
$\mathrm{CH}$ & & & \\
\hline
\end{tabular}

[1] J. L. Duncan, J. Mol. Struct. 6, 4,47 (1970).

[2] R. H. Schwendeman and J. D. Kelly, J. Chem. Phys. 42, 1132 (1965).

\section{Bromomethylmercury}

\begin{tabular}{|c|c|c|c|c|}
\hline $\mathrm{CH}_{3} \mathrm{BrHg}$ & & $\mathrm{H}_{3} \mathrm{CHgBr}$ & & $\mathrm{C}_{3 \mathrm{v}}$ \\
\hline Bond & Substitution & Effective & Angle & Efreclive \\
\hline $\begin{array}{l}\mathrm{HgC}_{\mathrm{gC}} \\
\mathrm{Hg}_{\mathrm{B}} \\
\mathrm{CH}\end{array}$ & $\begin{array}{l}2.072 \mathrm{C} \\
2.106 \mathrm{C}\end{array}$ & $\begin{array}{l}2.061 \mathrm{C} \\
2.505 \mathrm{C} \\
1.095 \mathrm{C}\end{array}$ & $\mathrm{HCH}$ & $109.6^{\circ} \mathrm{C}$ \\
\hline
\end{tabular}

[1] C. Walls, D. G. Lister and J. Sheridan, J. Chem. Soc. Faraday Trans., $\mathbf{7 1}, 1091$ (1975).

[2] W. Gordy and J. Sheridan, J. Chem. Phys. 22, 92 (1954). 
Choromethane

\begin{tabular}{lrr|ll}
\hline \hline Bond & Substitution & Equilibrium & Angle & Equilibrium \\
\hline $\begin{array}{l}\mathrm{CCl} \\
\mathrm{CH}\end{array}$ & $1.781 \mathrm{~B}$ & $\begin{array}{l}1.778 \mathrm{~B} \\
1.086 . \mathrm{C}\end{array}$ & HCH & $110.7 \mathrm{C}$ \\
\hline
\end{tabular}

[1] J. I. Duncan, J. Mol. Struct. 6, 447 (1970).

[2] S. L. Miller, L. C. Aamodt, G. Dousmanis, C. H. Townes and J. Kraitchman, J. Chem. Phys. 20, 1112 (1952).

[3] R. H. Schwendeman and J. D. Kelly, J. Chem. Phys, 42, 1132 (1965).

\section{Chloromethylmercury}

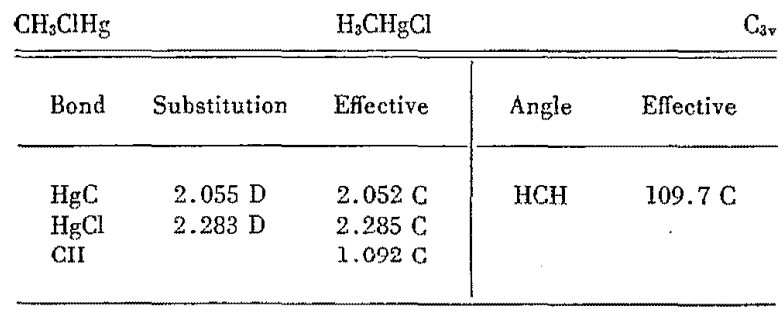

[1] C. Walls, D. G. Lister and J. Sheridan, J. Chem. Soc. Faraday Trans., 71, 1091 (1975).

[2] J. T. Cox, T. Guumann and W. J. Orville-Thomas, Disc. Faraday Soc. 19, 52 (1955).

[3] W. Gordy and J. Sheridan, J. Chem. Phys. 22, 92 (1954).

\section{Methyl hypochlorite}

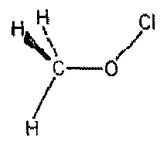

$\mathrm{CH}_{3} \mathrm{ClO}$

\begin{tabular}{ll|ll}
\hline Bond & Effective & Angle & Effective \\
\hline & & & \\
$\mathrm{CH}$ & $1.101 \mathrm{D}$ & $\mathrm{COCl}$ & $110.5 \mathrm{D}$ \\
$\mathrm{CO}$ & $1.418 \mathrm{C}$ & $\mathrm{HCH}$ & $111.3 \mathrm{D}$ \\
$\mathrm{OCl}$ & $1.690 \mathrm{C}$ & & \\
\hline
\end{tabular}

The $\mathrm{CH}_{3}$ group is tilted $5.2^{\circ}$ away from the chlorine atom, and thas becn assumed to be symmetric. The parameters were rocaleulated from the original rotational constants.

[1] J. S. Rigden and S. S. Butcher, J. Chem. Phys. 40, 2109 (1964).
Methanesulfenyl chloride

\begin{tabular}{lll|ll}
$\mathrm{CH}_{3} \mathrm{ClS}$ & & $\mathrm{H}_{3} \mathrm{CsCl}$ & & $\mathrm{C}_{*}$ \\
\hline \hline Bond & Substitution & Effective & Angle & Effective \\
\hline $\mathrm{SCl}$ & $2.030 \mathrm{~B}$ & $2.037 \mathrm{~B}$ & ClsC & $99.4 \mathrm{~B}$ \\
$\mathrm{CS}$ & & $1.788 \mathrm{~B}$ & & \\
$\mathrm{CH}$ & & $1.082 \mathrm{C}$ & $\theta^{\mathrm{a}}$ & $110.2 \mathrm{X}$ \\
\hline
\end{tabular}

Methyl group was assumed to be symmetrical, and to be staggered with respect to $\mathrm{SCl}$ bond.

a Angle between in-plane CH bond and methyl top axis.

[I] A. Guarnieri, Z. Charpentier and B. Kuck, Z. Füaturfursch. 28a, 1721 (1973).

[2] A. Cuarnieri, Z. Naturforsch. 23a, 1867 (1968).

\section{Methyltrichlorogermane}

\begin{tabular}{cc|cc}
$\mathrm{CH}_{3} \mathrm{Cl}_{3} \mathrm{Ge}$ & $\mathrm{H}_{3} \mathrm{CGeCl}_{3}$ & $\mathrm{C}_{3 \mathrm{~V}}$ \\
\hline \hline Bond & Substitution & Angle & Substitution \\
\hline $\mathrm{CcCl}$ & $2.135 \mathrm{C}$ & $\mathrm{CCeCl}$ & $106.0 \mathrm{C}$
\end{tabular}

[1] J. R. Durig, P. J. Cooper and Y. S. Li, J. Mol. Spectrose. 57, 169 (1975).

\section{Methyltrichlorosilane}

\begin{tabular}{ccc}
$\mathrm{CH}_{3} \mathrm{Cl}_{3} \mathrm{Si}$ & $\mathrm{H}_{3} \mathrm{C}-\mathrm{SiCl}_{3}$ & $\mathrm{C}_{3 \mathrm{r}}$ \\
\hline \hline Bond & Effective \\
\hline $\mathrm{SiC}$ & $1.876 \mathrm{X}$ \\
$\mathrm{SiCl}$ & $2.021 \mathrm{X}$
\end{tabular}

Methyl group was assumed to be tetrahedral with $\mathrm{CH}=1.093$, and angle $\mathrm{ClSiCl}$ was fixed at 109.4 .

[1] R. C. Mockler, J. H. Bailey and W. Gordy, J. Chem. Phys. 21, 1710 (1953).

[2] M. Mitzlaff, R. Holm and H. Hartmann, Z. Naturforsch. 22, 1415 (1967)

\section{Fluoromethane}

\begin{tabular}{cc|cc}
$\mathrm{CH}_{3} \mathrm{~F}$ & & $\mathrm{C}_{8 \mathrm{v}}$ \\
\hline \hline Bond & Substitution & Angle & Substitution \\
\hline $\mathrm{CF}$ & $1.383 \mathrm{~A}$ & HCll & $110.6 \mathrm{~B}$ \\
$\mathrm{CH}$ & $1.100 \mathrm{~B}$ & &
\end{tabular}

[1] W. W. Clark and F. C. DeLucial, J. Mol. Struct. 32, 29 (1976). 
Methanesulfonyl Fluoride

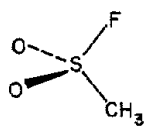

$\mathrm{CH}_{3} \mathrm{FO}_{2} \mathrm{~S}$

Exccpt for symmetry no struetural results wero obtainod.

[1] E. J. Jacob and D. R. Lide, J. Chem. Phys, 54, 4591 (1971).

N,N-Difluoromethylamine

\begin{tabular}{|c|c|c|c|}
\hline \multicolumn{4}{|l|}{$\mathrm{CH}_{3} \mathrm{~F}_{2} \mathrm{~N}$} \\
\hline Bond. & Effective & Angle & Fiffective \\
\hline $\mathrm{CH}$ & $1.091 \mathrm{C}$ & FNF & $101 \mathrm{E}$ \\
\hline $\mathrm{CN}$ & $1.45 \mathrm{E}$ & CNF & $105 \cdot E$ \\
\hline \multirow[t]{4}{*}{$\mathrm{NF}$} & $1.41 \mathrm{E}$ & $\mathrm{NCH}$ & $110 \mathrm{E}$ \\
\hline & & $\mathrm{NCH}^{\prime}$ & $106 \mathrm{E}$ \\
\hline & & $\mathrm{HCH}^{\prime}$ & $112 \mathrm{E}$ \\
\hline & & $\mathrm{HCH}$ & $110 \mathrm{E}$ \\
\hline
\end{tabular}

$\mathrm{CH}$ and $\mathrm{CH}^{\prime}$ assumed equal.

$\mathrm{CH}_{3}$ and $\mathrm{NF}_{2}$ group are staggered.

[I] L. Pierce. R. Hayes, and J. Beecher, J. Chem. Phys. 46, 4352 (1967).

Phosphorodifluoridous acid methyl ester

(Methoxydifluorophosphine)

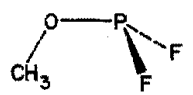

$\mathrm{CH}_{3} \mathrm{~F}_{2} \mathrm{OP}$

\begin{tabular}{ll|lr}
\hline Bond & Effective & Angle & Effective \\
\hline & & & \\
\hline $\mathrm{PF}$ & $1.591 \mathrm{C}$ & $\mathrm{FPF}$ & $94.8 \mathrm{D}$ \\
$\mathrm{PO}$ & $1.560 \mathrm{D}$ & $\mathrm{OPF}$ & $102.2 \mathrm{D}$ \\
$\mathrm{CO}^{\mathrm{s}}$ & $1.446 \mathrm{~B}$ & $\mathrm{COP}$ & $123.7 \mathrm{C}$ \\
$\mathrm{CH}$ & $1.090 \mathrm{C}$ & $\mathrm{HCH}$ & $110.5 \mathrm{D}$ \\
\hline
\end{tabular}

a This distance is $r_{\mathrm{s}}$.

[1] E. G. Codding, C. E. Jones and R. H. Schwendeman, Inorg. Chem. 13, 178 (1974).
Methyldifluorophosphine

\begin{tabular}{cc|cc}
$\mathrm{CH}_{3} \mathrm{~F}_{2} \mathrm{P}$ & \multicolumn{2}{c}{$\mathrm{CH}_{3} \mathrm{PF}_{2}$} & $\mathrm{C}_{\mathrm{B}}$ \\
\hline \hline Bond & Effective & Angle & Effective \\
\hline & & & \\
$\mathrm{CH}$ & $1.093^{\mathrm{a}}$ & $\mathrm{PCH}$ & $109.7^{\mathrm{a}}$ \\
$\mathrm{PF}$ & $1.582^{\mathrm{a}}$ & $\mathrm{FPF}$ & $98.4 \mathrm{C}$ \\
$\mathrm{PC}$ & $1.825 \mathrm{D}$ & $\mathrm{FPC}$ & $97.8 \mathrm{C}$ \\
\hline
\end{tabular}

Assumed values.

[1] E. G. Codding, R. A. Creswell and R. H. Schwendeman, Inorg. Chem., 13, 856 (1974).

Methyltrifluorosilane

\begin{tabular}{ccc|cc}
$\mathrm{CH}_{3} \mathrm{~F}_{3} \mathrm{Si}$ & & $\mathrm{H}_{3} \mathrm{CSiF}_{3}$ & & $\mathrm{C}_{3 \mathrm{r}}$ \\
\hline \hline Bond & Substitution & Effective & Angle & Effective \\
& & & & \\
\hline $\mathrm{CH}$ & $1.081 \mathrm{~B}$ & & $\mathrm{HCSi}$ & $111.0 \mathrm{~B}$ \\
$\mathrm{CSi}$ &. & $1.812 \mathrm{D}$ & FSiC & $112.3 \mathrm{D}$ \\
$\mathrm{SiF}$ & & $1.574 \mathrm{C}$ & & \\
\hline
\end{tabular}

[1] J. R. Durig, Y. S. Li, and C. C. Tong, J. Mol. Struct. 14, 255 (1972).

\section{Germyl Cyanide}

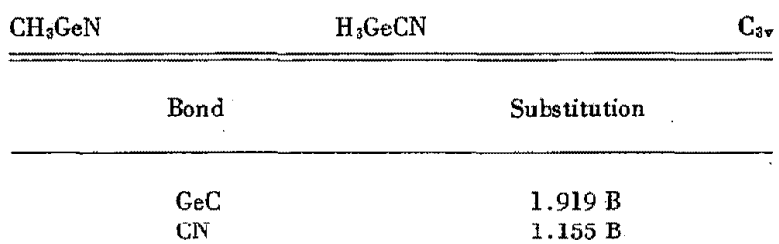

[1] R. Varma and K. S. Buckton, J. Chem. Phys., 46, 1565 (1967).

\section{lodomethylmercury}

\begin{tabular}{|c|c|c|c|c|}
\hline $\mathrm{CH}_{3} \mathrm{HgI}$ & & $\mathrm{H}_{3} \mathrm{CHgI}$ & & $\mathrm{C}_{3 \mathrm{v}}$ \\
\hline Bond & Substitution & Effective & Angle & Effective \\
\hline $\mathrm{HgC}$ & $2.077 \mathrm{C}$ & $2.069 \mathrm{C}$ & \multirow[t]{3}{*}{$\mathrm{HCH}$} & \multirow{3}{*}{$109.6 \mathrm{C}$} \\
\hline $\mathrm{HgI}$ & $2.571 \mathrm{C}$ & $2.588 \mathrm{C}$ & & \\
\hline $\mathrm{CH}$ & & $1.095 \mathrm{C}$ & & \\
\hline
\end{tabular}

[1] C. Walls, D. G. Lister, and J. Sheridan, J. Chem. Soc., Faraday Trans., 71, 1091 (1975). 
Methyl lodide

$\mathrm{CH}_{3} \mathrm{I}$

\begin{tabular}{cc|cc}
\hline Bond & Equilibrium & Angle & Equilibrium \\
\hline $\mathrm{CH}$ & $1.084 \mathrm{~B}$ & $\mathrm{HCH}$ & $111.2 \mathrm{~B}$ \\
$\mathrm{CI}$ & $2.132 \mathrm{~B}$ & & \\
\hline
\end{tabular}

[1] H. Matsuura and J. Overend, J. Chem. Phys. 56, 5725 (1972).

[2] H. Matsuura and J. Overend, Spectrochim. Acta 27 A, 2165 (1971).

[3] H. Matsuura, T. Nakagawa and J. Overend, J. Chem. Phys. 59, 1449 (1973)

[4] R. J. L. Popplewell and H. W. Thompson, Spectrochim. Acta 25A, 287 (1969).

[5] T. L. Barnet: and T. H. Edwards, J. Mol. Spectrosc. 23, 302 (1967).

[6] Y. Morino and C. Hirose, J. Mol. Spectrosc. 22, 99 (1967).

[7] T. E. Sullivan and L. Frenkel, J. Mol. Spectrosc. 39, 185 (1971).

[O] R. W. Petcrson and T. II. Edvards, J, Mol, Spectrosc. 38, I (1971).

[9] E. W. Jones, R. J. L. Popplewell and H. W. Thompson, Proc. Roy. Soc. A288, 39 (1965).

[10] J, W. Simmons and J. H. Conthetein, J. Chem. Phys 20, 122 (1952).

$\begin{array}{lllll} & & & \\ \\ \end{array}$

Small $\ddot{b}$ coordinates of $\mathrm{C}$ and $\mathrm{N}$ leau to large structural uncertainties.

[1] R. Pearson, Jr. and F. J. Lovas, J. Chem. Phys. 66, 4.149 (1977).

[2] D. R. Johnson and F. J. Lovas, Chem. Phys. Lett. i5, 65 (1972).

\section{Formoldoxime}

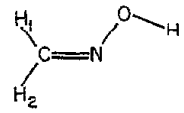

$\mathrm{CH}_{3} \mathrm{NO}$

\begin{tabular}{llll}
\hline Bond & Substitution & Angle & Substitution \\
\hline & $1.276 \mathrm{~B}$ & $\mathrm{H}_{1} \mathrm{CN}$ & $121.8 \mathrm{C}$ \\
$\mathrm{C}-\mathrm{N}$ & $1.408 \mathrm{~B}$ & $\mathrm{H}_{2} \mathrm{CN}$ & $115.6 \mathrm{C}$ \\
$\mathrm{NO}$ & $0.956 \mathrm{~B}$ & $\mathrm{CNO}$ & $110.2 \mathrm{C}$ \\
$\mathrm{OH}$ & $1.085 \mathrm{C}$ & $\mathrm{NOH}$ & $102.7 \mathrm{C}$ \\
$\mathrm{CH}_{1}$ & $1.086 \mathrm{C}$ & & \\
$\mathrm{CH}_{2}$ & & & \\
\hline
\end{tabular}

[1] I. N. Levine, J. Chem. Phys., 38, 2326 (1963).

[2] I. N. Levine, J. Mol. Spectr., 8, 276 (1962).

\section{Formomide}

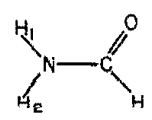

$\mathrm{CH}_{3} \mathrm{NO}$

\begin{tabular}{ll|ll}
\hline Bond & Substitution & Angle & Substitution \\
\hline & & & \\
$\mathrm{CN}$ & $1.352 \mathrm{D}$ & $\mathrm{H}_{1} \mathrm{NH}_{2}$ & $121.6 \mathrm{C}$ \\
$\mathrm{CO}$ & $1.219 \mathrm{D}$ & $\mathrm{H}_{1} \mathrm{NC}$ & $118.5 \mathrm{C}$ \\
$\mathrm{CH}$ & $1.098 \mathrm{D}$ & $\mathrm{NCO}$ & $124.7 \mathrm{C}$ \\
$\mathrm{NH}_{1}$ & $1.002 \mathrm{C}$ & $\mathrm{NCH}$ & $112.7 \mathrm{D}$ \\
$\mathrm{NH}_{2}$ & $1.002 \mathrm{C}$ & & \\
\hline
\end{tabular}

The molecule has been assumed to be planar, with a low-frequency single-minimum amino wagging potential function. Several small coordinates cause additional difficulties. The structure results above are a hybrid of $-\mathrm{NH}_{2}$ and $-\mathrm{ND}_{2}$ results.

IIT E. Hirote, R. Sugisaki, C. J. Nielsen and G. O. Sorensen, J. Mol. Spectroso. 49, 251 (1974)

[2] C. C. Costain and J. M. Dowling, J. Chem. Phys. 32, 158 (1960). [3] R. J. Kurland and E. B. Wilson, Jr., J. Chem. Phys. 27, 585 (1957).

\section{Nitrosomethane}

$\mathrm{CH}_{3} \mathrm{NO}$

$$
\operatorname{ll}(\mathrm{C}-\mathrm{N} \quad 1)
$$

Isotopic substitution slanel that the methyl group eclipsed the $N=0$ bond.

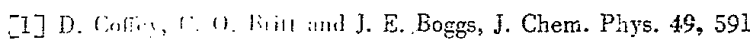
$: \operatorname{lig} i s i$ 
Methyl Thionylamine

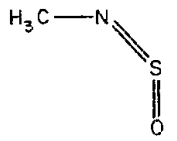

Methyl Nitrate

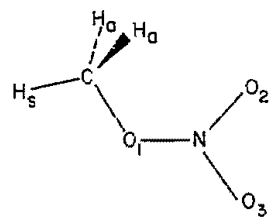

$\mathrm{CH}_{3} \mathrm{NOS}$

\begin{tabular}{cc}
\hline \hline Angle & Effective \\
\hline CNS & $122 \mathrm{X}$
\end{tabular}

Only one isotopic species was studied.

[1: V. M. Rao, J. T. Yardley, and R. F. Curl, Jr., J. Chem. Phys. 42,284 (1965).

\section{Isocyanatosilane}

\begin{tabular}{cc|cc}
$\mathrm{CH}_{9} \mathrm{NOSi}$ & \multicolumn{2}{c}{$\mathrm{H}_{3} \mathrm{SiN}=\mathrm{C}=0$} & $\mathrm{C}_{3 \mathrm{r}}$ \\
\hline \hline Bond & Substitution & Angle & Substitution \\
\hline $\mathrm{CO}$ & $1.179 \mathrm{~A}$ & HSill & $110.4 \mathrm{D}$ \\
$\mathrm{CN}$ & $1.150 \mathrm{E}$ & & \\
$\mathrm{NSi}$ & $1.699 \mathrm{E}$ & & \\
$\mathrm{SiH}$ & $1.506 \mathrm{C}$ & &
\end{tabular}

[1] M. C. L. Gerry, J. C. Thompson, and T. M. Sugden, Nature 211, 846 (1966).

Nitromethane

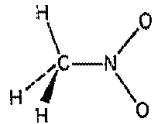

$\mathrm{CH}_{3} \mathrm{NO}_{2}$

\begin{tabular}{ll|ll}
\hline Bond & Substitution & Angle & Substitution \\
\hline CN & $1.489 \mathrm{~B}$ & ONO & $125.3 \mathrm{~B}$ \\
NO & $1.22 \mathrm{~B}$ & $\mathrm{NCH}$ & $107.2 \mathrm{~B}$ \\
\hline
\end{tabular}

$\mathrm{CH}$ bond length was assumed $(1.088 \AA)$.

[1] A. P. Cox and S. Waring, Trans. Faraday Soc. 68, 1060 (1972)
C. $\quad \mathrm{CH}_{3} \mathrm{NO}_{3}$

\begin{tabular}{ll|ll}
\hline Bond & Substitution & Angle & Substitution \\
\hline & & & \\
$\mathrm{CH}_{3}$ & $1.095 \mathrm{C}$ & $\mathrm{H}_{\mathrm{a}} \mathrm{CH}_{\mathrm{a}}$ & $109.8 \mathrm{C}$ \\
$\mathrm{CH}_{3}$ & $1.088 \mathrm{C}$ & $\mathrm{H}_{8} \mathrm{CO}$ & $103.4 \mathrm{C}$ \\
$\mathrm{CO}$ & $1.437 \mathrm{~B}$ & $\mathrm{H}_{\mathrm{a}} \mathrm{CO}$ & $110.4 \mathrm{C}$ \\
$\mathrm{NO}_{1}$ & $1.402 \mathrm{~B}$ & $\mathrm{CON}$ & $112.72 \mathrm{~B}$ \\
$\mathrm{NO}_{2}$ & $1.205 \mathrm{~B}$ & $\mathrm{O}_{1} \mathrm{NO}_{2}$ & $118.10 \mathrm{~B}$ \\
$\mathrm{NO}_{3}$ & $1.208 \mathrm{~B}$ & $\mathrm{O}_{2} \mathrm{NO}_{3}$ & $129.52 \mathrm{~B}$ \\
& & & \\
\hline
\end{tabular}

Heavy atoms are coplanar. Methyl group is tilted away from $O_{2}$ by $4.8 \pm 1^{\circ}$. Axis of $\mathrm{NO}_{2}$ group is also tilted away from $\mathrm{CH}_{3}$ group by $2.9^{\circ}$. $s$ and a refer to in-plane and out-of-plane, respectively.

[1] A. P. Cox and S. Waring, Trans. Faraday Soc. 67, 3441 (1971),

[2] W. B. Dixon and E. B. Wilson, J. Chem. Phys. 35, 191 (1961).

\section{Thioformamide}

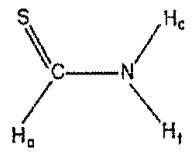

$\mathrm{CH}_{3} \mathrm{NS}$

$\mathrm{C}_{\mathrm{s}}$

\begin{tabular}{|c|c|c|c|}
\hline Bond & Substitution & Angle & Substitution \\
\hline $\mathrm{NH}_{\mathrm{e}}$ & $1.002 \mathrm{C}$ & $\mathrm{H}_{\mathrm{c}} \mathrm{NH}_{\mathrm{t}}$ & 120 \\
\hline $\mathrm{NH}_{\mathrm{t}}$ & $0.99 \mathrm{D}$ & $\mathrm{H}_{e} \mathrm{NC}$ & $117.9 \mathrm{~B}$ \\
\hline $\mathrm{CN}$ & $1.358 \mathrm{~B}$ & $\mathrm{H}_{t} \mathrm{NC}$ & $\exists 22 \quad \mathrm{D}$ \\
\hline $\mathrm{CS}$ & $1.626 \mathrm{~B}$ & NCS & $125.3 \mathrm{~F}$ \\
\hline \multirow[t]{2}{*}{$\mathrm{CH}_{\mathrm{s}}$} & $1.12 \mathrm{E}$ & $\mathrm{NCH}_{\mathbf{a}}$ & $108 \mathrm{D}$ \\
\hline & & $\mathrm{SCH}_{\mathrm{a}}$ & $127 \quad \mathrm{D}$ \\
\hline
\end{tabular}

Several parameters have been recomputed from reported data. [1] R. Sugisaki, T. Tanaka, and E. Hirota, J. Mol. Spectrose. 49, 241 (1974). 
Isothiocyanatosilane

\begin{tabular}{cc|cc}
$\mathrm{CH}_{3} \mathrm{NSSi}$ & \multicolumn{2}{c}{$\mathrm{H}_{3} \mathrm{SiNCS}$} & $\mathrm{C}_{3 \mathrm{v}}$ \\
\hline \hline Bond & Substitution & Angle & Substitution \\
\hline $\mathrm{CN}$ & $1.211 \mathrm{C}$ & $\mathrm{HSiH}$ & $111.37 \mathrm{~B}$ \\
$\mathrm{SiN}$ & $1.714 \mathrm{C}$ & & \\
$\mathrm{SiH}$ & $1.489 \mathrm{~B}$ & & \\
\hline
\end{tabular}

CS bond was assumed.

[I] D. R. Jenkins, R. Kewley, and T. M. Sugden, Trans. Faraday Soc. 58, I284 (1962).

\section{Silanecarbonitrile}

\begin{tabular}{cccc}
$\mathrm{CH}_{3} \mathrm{NSi}$ & $\mathrm{SiH}_{3} \mathrm{CN}$ & $\mathrm{C}_{3 v}$ \\
\hline Bond & Effective & Angle & Effective \\
& & & \\
$\mathrm{SiH}$ & $1.49 \mathrm{E}$ & $\mathrm{CSiH}$ & $107.5 \mathrm{D}$ \\
$\mathrm{SiC}$ & $1.847 \mathrm{C}$ & &
\end{tabular}

CN bond was assumed.

[1] J. Sheridan and A. C. Turner, Proc. Chem. Soc. 1960, 21 (1960).

[2] N. Muller and R. C. Bracken, J. Chem. Phys, 32, 1577 (1960).

\begin{tabular}{lll}
\multicolumn{1}{c}{ Methane } & \\
\hline \hline Bond & Effective & Substitution \\
\hline & & \\
\hline $\mathrm{CH}$ & $1.094 \mathrm{~A}$ & $1.092 \mathrm{~A}$
\end{tabular}

The $r_{\theta}$ distance was calculated from the $B_{n}$ value of Ref. 1 . The substitution distance was calculated from the data of Ref. 1 and the $A_{0}$ and $B_{0}$ reported in Ref. 2 for $\mathrm{CH}_{3} \mathrm{D}$.

[I] G. Tarrago, M. Dang-Nhu and G. Poussigue, J. Mol. Spectrosc. 49, $322(1974)$

[2] W. B. Olson, J. Mol. Spectrosc. 43, 190 (1972).
N-Chloromethylamine

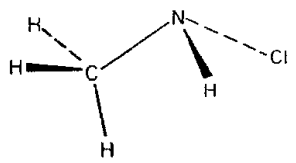

$\mathrm{CH}_{4} \mathrm{ClN}$

\begin{tabular}{cc|cc}
\hline Bond & Eflective & Angle & Effective \\
\hline $\mathrm{CN}$ & $1.475 \mathrm{C}$ & $\mathrm{CNCl}$ & $109.4 \mathrm{C}$ \\
$\mathrm{NCl}$ & $1.750 \mathrm{C}$ & & \\
\hline
\end{tabular}

Deuterium substitution indicated a staggered methyl group con. formation as shown in the figure.

[1] A. M. Mirri and W. Caminati, J. Mol. Spectrosc. 47, 204 (1973).

[2] W. Caminati, R. Cervellati and A. M. Mirri, J. Mol. Spectrosc. 51. 288 (1974).

\section{Methyldichlorosilane}

\begin{tabular}{cl|ll}
$\mathrm{CH}_{4} \mathrm{Cl}$ Si & \multicolumn{2}{c}{$\mathrm{CH}_{3} \mathrm{SiHCl}_{2}$} & $\mathrm{C}_{3}$ \\
\hline \hline \multirow{2}{*}{ Bond } & Effective & Angle & Effective \\
& & & \\
\hline $\mathrm{SiC}$ & $1.850^{\circ}$ & $\mathrm{SiCH}$ & $109.5^{\mathrm{a}}$ \\
$\mathrm{SiH}$ & $1.467^{\mathrm{a}}$ & $\mathrm{CSiH}$ & $110.9^{\mathrm{a}}$ \\
$\mathrm{CH}$ & $1.093^{\mathrm{a}}$ & $\mathrm{CSiCl}$ & $109.8 \mathrm{X}$ \\
$\mathrm{SiCl}$ & $2.040 \mathrm{X}$ & $\mathrm{CiSiCl}$ & $108.8 \mathrm{X}$ \\
\hline
\end{tabular}

Methyl group was assumed to be symmetrical.

- Assumed values.

[1] K. Endo, H. Takeo and C. Matsumura, Bull. Chem. Soc. Japan 50, 626 (1977).

Methyldifluorosilane

$\mathrm{CH}_{4} \mathrm{~F}_{2} \mathrm{Si} \quad \mathrm{CH}_{3} \mathrm{SiF}_{2} \mathrm{H} \quad \mathrm{C}_{8}$

\begin{tabular}{ll|ll}
\hline Bond & Substitution & Angle & Substitution \\
\hline & & & \\
\hline $\mathrm{SiC}$ & $1.840 \mathrm{C}$ & $\mathrm{FSiF}$ & $107.1 \mathrm{~B}$ \\
$\mathrm{SiF}$ & $1.580 \mathrm{C}$ & $\mathrm{CSiH}$ & $115.53 \mathrm{C}$ \\
$\mathrm{SiH}$ & $1.471 \mathrm{C}$ & $\mathrm{CSiF}$ & $109.37 \mathrm{~B}$ \\
$\mathrm{CH}$ & $1.094 \mathrm{~B}$ & $\mathrm{HCH}$ & $108.53 \mathrm{~B}$ \\
\hline
\end{tabular}

Symmetrical $\mathrm{CH}_{3}$ group assumed.

[1] L. C. Krisher and L. Pierce, J. Chem. Phys. 32, 1619 (1960).

[2] J. D. Swalen and B. P. Stoicheff, J. Chem. Phys. 28, 67I (1958). 
trans-Methyldiimide

$\mathrm{CH}_{4} \mathrm{~N}_{2}$

$\mathrm{CH}_{3} \mathrm{NNH}$

Insufficient data for structure determination. Only trans conformer found.

[1] W. Steinmetz, J. Chem. Phys. 52, 2788 (1970),

\section{Methanol}

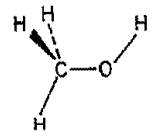

$\mathrm{CH}_{4} \mathrm{O}$

\begin{tabular}{lll|llll}
\hline \hline Bond & Effective & Substitution & Angle & Effective & Substitution \\
\cline { 3 - 6 } & & & & & \\
$\mathrm{CH}$ & $1.094 \mathrm{~B}$ & $1.094 \mathrm{~B}$ & $\mathrm{HCH}$ & $108.6 \mathrm{C}$ & $108.5 \mathrm{~B}$ \\
$\mathrm{OH}$ & $0.945 \mathrm{~B}$ & $0.963 \mathrm{C}$ & $\mathrm{COH}$ & $108.5 \mathrm{~B}$ & $108.0 \mathrm{~B}$ \\
$\mathrm{CO}$ & $1.425 \mathrm{~B}$ & $1.421 \mathrm{~A}$ & $\phi^{\mathrm{s}}$ & $3.3 \mathrm{~B}$ & $3.2 \mathrm{~B}$
\end{tabular}

${ }^{8}$ Angle between $\mathrm{CH}_{3}$ internal rotation axis and $\mathrm{CO}$ line; tilt is away from $\mathrm{OH}$ group.

[1] M. C. L. Gerry and R. M. Lces, J. Mol. Spectrose, 6l, 231 (1976).

[2] R. M. Lees and J. G. Baker, J. Chem. Phys. 48, 5299 (1968).

[3] Y. Y. Kwan and D. M. Dennison, J. Mol. Spectrose. 43, 291 (1972).

Methonethiol

\begin{tabular}{cc|cr}
$\mathrm{CH}_{4} \mathrm{~S}$ & & $\mathrm{CH}_{3} \mathrm{SH}$ & $\mathrm{C}_{\mathrm{B}}$ \\
\hline \multirow{2}{*}{ Bond } & Effective & Angle & Effective \\
& & & \\
$\mathrm{SH}$ & $1.355 \mathrm{X}$ & $\mathrm{CSH}$ & $96.5 \mathrm{X}$ \\
$\mathrm{CS}$ & $1.819 \mathrm{X}$ & $\mathrm{HCH}$ & $109.75 \mathrm{X}$ \\
$\mathrm{CH}$ & $1.092 \mathrm{X}$ & Methyl tilt & $2.17 \mathrm{X}$ \\
\hline
\end{tabular}

a See methanol for definition

[1] T. Kojima, J. Phys. Soc. Japan 15, 1284 (1960).

\section{Methaneselenol}

\begin{tabular}{cc|cr}
$\mathrm{CH}_{4} \mathrm{Se}$ & \multicolumn{2}{c}{$\mathrm{CH}_{3} \mathrm{SeH}$} & $\mathrm{C}_{3}$ \\
\hline \hline Bond & Effective & Angle & Hfiective \\
\hline $\mathrm{SeH}$ & $1.47 \mathrm{X}$ & $\mathrm{CSeH}$ & $95.4 \mathrm{X}$ \\
$\mathrm{CSe}$ & $1.96 \mathrm{X}$ & $\mathrm{HCH}$ & $110.0 \times$ \\
$\mathrm{CH}$ & $3.09 \mathrm{X}$ & & \\
& & & \\
\hline
\end{tabular}

Internal rotation analysis indicated methyl group tilt of $1.5 \pm$ $1.0^{\circ}$. See methanol for definition.

[1] C. H. Thomas, J. Chem. Phys. 59, 70 (1974).

Chloromethylsilane

$\mathrm{C}_{\mathrm{H}}$

\begin{tabular}{cc|cc}
$\mathrm{CH}_{5} \mathrm{ClSi}$ & \multicolumn{2}{c}{$\mathrm{CH}_{2} \mathrm{ClSiH}_{3}$} & $\mathrm{C}_{\mathrm{B}}$ \\
\hline \multirow{2}{*}{ Bond } & Substitution & Angle & Substitution \\
\hline & & & \\
$\mathrm{CSi}$ & $1.889 \mathrm{C}$ & $\mathrm{SiCCl}$ & $109.3 \mathrm{C}$ \\
$\mathrm{CCl}$ & $1.788 \mathrm{C}$ & $\mathrm{HSill}$ & $110.6 \mathrm{C}$ \\
$\mathrm{CH}$ & $1.096 \mathrm{C}$ & $\mathrm{HCH}$ & $107.5 \mathrm{C}$ \\
$\mathrm{SiH}$ & $1.477 \mathrm{~B}$ & $\mathrm{SiCH}$ & $109.3 \mathrm{C}$ \\
\hline
\end{tabular}

All Sill bonds assumed equal.

[1] R. H. Schwendeman and G. D. Jacobs, J. Chem. Phys. 36, 1251 (1962).

\section{Methyimonofluorogermane}

\begin{tabular}{|c|c|c|c|c|}
\hline $\mathrm{H}_{5} \mathrm{~F} \mathrm{Ge}$ & & $\mathrm{CH}_{3} \mathrm{GeH}_{2} \mathrm{~F}$ & & \\
\hline Bond & Substitution & Effective & Angle & Effective \\
\hline $\mathrm{CGe}$ & $1.925 \mathrm{~B}$ & & $\mathrm{FGeC}$ & $106.3 \mathrm{D}$ \\
\hline GeF & & $1.751 \mathrm{D}$ & $\mathrm{HCH}$ & $108.9 \mathrm{C}$ \\
\hline $\mathrm{CH}$ & & $1.094 \mathrm{C}$. & & \\
\hline
\end{tabular}

$\mathrm{GeH}=1.525 \AA, \mathrm{HGeH}=110^{\circ}$, and $\mathrm{CGeH}=108.9^{\circ}$ were assumed in order to determine $\mathrm{GeF}$ and $\mathrm{FGeC}$. The $\mathrm{CH}_{3}$ group was assumed to be symmetrical. There is cridenee from the internal rotation that the $\mathrm{CH}_{3}$ group is tilled $1.9^{\circ}$ toward the $\mathrm{F}$ atom.

[1] R. F. Roberts, R. Varma, and J. F. Nelson, J. Chem Phys. 64, $5035(1976)$

\section{Methylfuorosilane}

\begin{tabular}{cccc}
$\mathrm{CH}_{5} \mathrm{FSi}$ & \multicolumn{2}{c}{$\mathrm{CH}_{3} \mathrm{SiH}_{2} \mathrm{~F}$} & $\mathrm{C}_{\mathrm{s}}$ \\
\hline \multirow{2}{*}{ Bond } & Substitution & Angle & Substitution \\
& & & \\
\hline $\mathrm{SiC}$ & $1.849 \mathrm{~B}$ & $\mathrm{HSiH}$ & $110.0 \mathrm{~B}$ \\
$\mathrm{SiF}$ & $1.597 \mathrm{~B}$ & $\mathrm{CSiH}$ & $112.5 \mathrm{D}$ \\
$\mathrm{SiH}$ & $1.477 \mathrm{~B}$ & $\mathrm{HCH}$ & $108.5 \mathrm{~B}$ \\
$\mathrm{CH}$ & $1.099 \mathrm{~B}$ & $\mathrm{CSiF}$ & $108.9 \mathrm{~B}$ \\
& & & \\
\hline
\end{tabular}

Axial symmetry of $\mathrm{CH}_{3}$ group was assumed.

[1] L. C. Krishner and L. Pierce, J. Chem. Phys. 32, 1619 (1960).

$c$ 


\section{Methylamine}

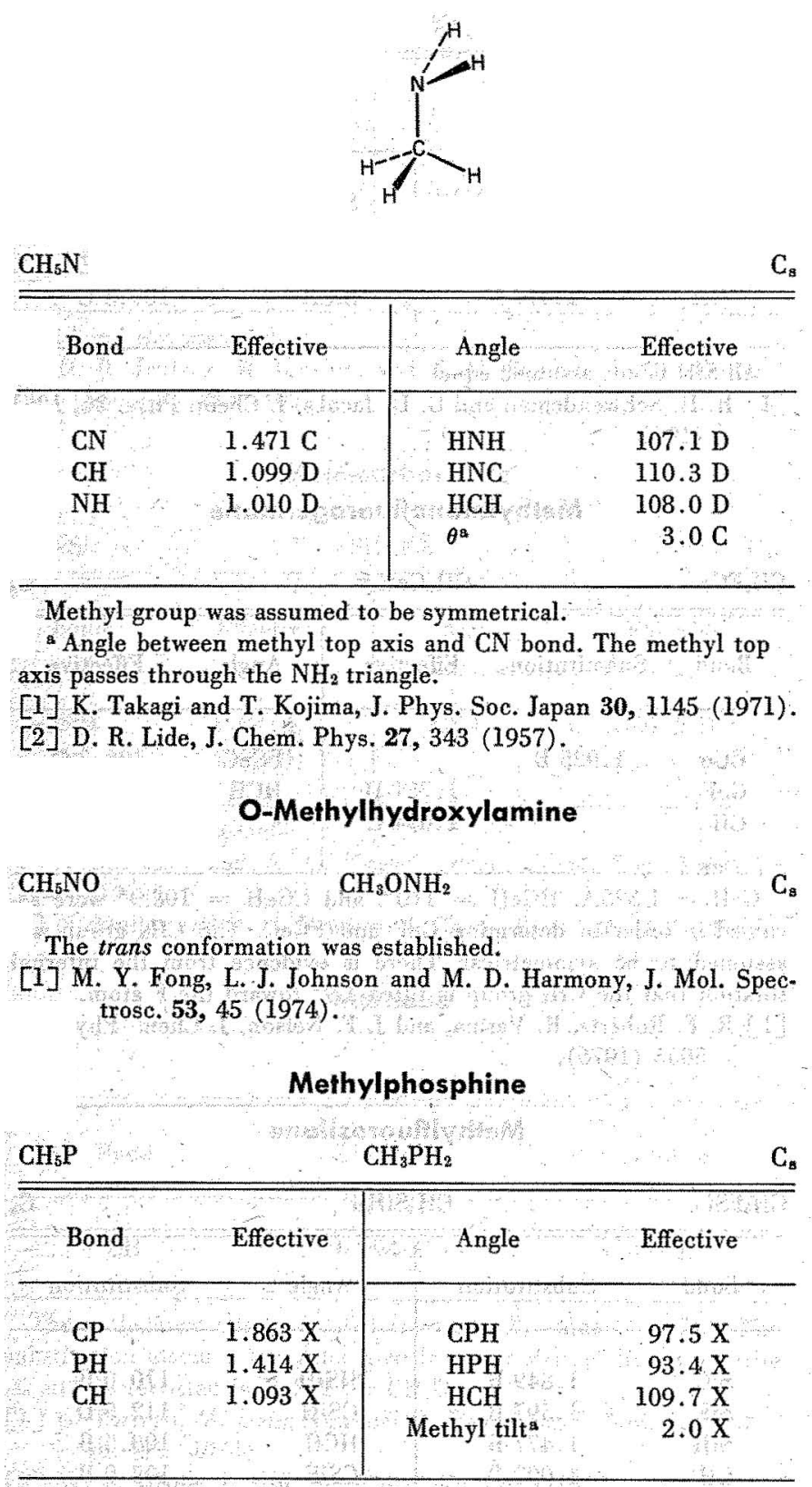

- $\mathrm{CH}_{3}$ tilted away from $\mathrm{PH}_{2}$ moiety.

[1] T. Kojima, E. L. Breig and C. C. Lin, J. Chem. Phys. 35, 2139 (1961)

\section{Methylgermane}

\begin{tabular}{ccc|ccc}
$\mathrm{CH}_{6} \mathrm{Ge}$ & \multicolumn{4}{c}{$\mathrm{CH}_{3} \mathrm{GeH}_{3}$} & \\
\hline & $\begin{array}{c}\text { Substitu } \\
\text { tion }\end{array}$ & Effective & Angle & $\begin{array}{c}\text { Substitu- } \\
\text { tion }\end{array}$ & Effective \\
Bond & & & & & \\
\hline $\mathrm{CH}$ & $1.083 \mathrm{~B}$ & $1.089 \mathrm{~B}$ & $\mathrm{HCH}$ & $108.4 \mathrm{C}$ & $108.2 \mathrm{C}$ \\
$\mathrm{GeH}$ & $1.529 \mathrm{~B}$ & $1.534 \mathrm{~B}$ & $\mathrm{HGeH}$ & $109.2 \mathrm{C}$ & $108.6 \mathrm{C}$ \\
$\mathrm{CGe}$ & $1.945 \mathrm{~A}$ & & & & \\
\hline
\end{tabular}

[1] V. W. Laurie, J. Chem. Phys. 30, 1210 (1959).

\section{Methylhydrazine}

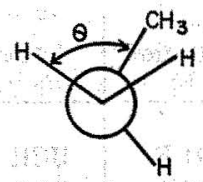

\begin{tabular}{ccc}
$\mathrm{CH}_{6} \mathrm{~N}_{2}$ & Inner Rotamer & $\mathrm{C}_{1}$ \\
\hline & Angle & Effective \\
& & \\
\hline & 0 & $-84.5 \mathrm{X}$ \\
& & \\
\hline
\end{tabular}

Two rotamers have been observed; an inner rotamer and an outer rotamer.

[1] R. P. Lattimer and M. D. Harmony, J. Chem. Phys. 53, 4575 (1970).

\section{Methylhydrazine}

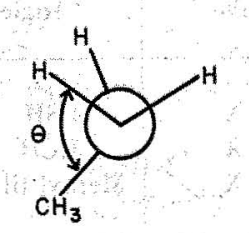

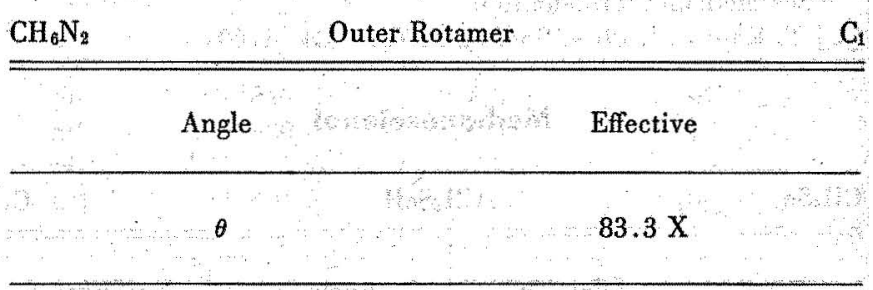

Two rotamers have been observed; an inner and an outer.

[1] R. P. Lattimer and M. D. Harmony, J. Chem. Phys. 53, 4575 (1970). 


\section{Mefhyisilane}

\begin{tabular}{lll|ll}
$\mathrm{CE}_{6} \mathrm{Si}$ & & $\mathrm{CH}_{3} \mathrm{SiH}_{3}$ & & $\mathrm{C}_{3 \gamma}$ \\
\hline Bond & Substitution & Effective & Angle & Effective \\
\hline & & & $\mathrm{HCH}$ & $107.7 \mathrm{C}$ \\
$\mathrm{SiC}$ & $1.869 \mathrm{~A}$ & & $\mathrm{HSiH}$ & $108.2 \mathrm{C}$ \\
$\mathrm{CH}$ & & $1.093 \mathrm{~B}$ & $\mathrm{H}$ & \\
$\mathrm{SiH}$ & & $1.485 \mathrm{~B}$ & & \\
\hline
\end{tabular}

[1] R. W. Kilb and L. Pierce, J. Chem. Phys. 27, 108 (1957).

Methylstannañe

\begin{tabular}{ll|ll}
$\mathrm{CH}_{6} \mathrm{Sn}$ & \multicolumn{2}{c}{$\mathrm{CH}_{3} \mathrm{SnH}_{3}$} & $\mathrm{C}_{3 \mathrm{v}}$ \\
\hline \hline Bond & Effective & Angle & Effective \\
\hline $\mathrm{CSn}$ & $2.143 \mathrm{X}$ & $\mathrm{HCH}$ & $109.5 \mathrm{X}$ \\
$\mathrm{SnH}$ & $1.700 \mathrm{X}$ & $\mathrm{HSnH}$ & $109.5 \mathrm{X}$ \\
$\mathrm{CH}$ & $1.09 \mathrm{X}$ & & \\
\hline
\end{tabular}

[1] D. R. Lide, Jr., J. Chem. Phys. 19, 1605 (1951).

\section{Monocarbahexaborane}

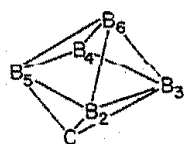

$\mathrm{CH}_{7} \mathrm{~B}_{5}$

\begin{tabular}{ll}
\hline \hline Bond & Substitution \\
\hline & \\
$\mathrm{B}_{2} \mathrm{~B}_{3}$ & $1.872 \mathrm{~B}$ \\
$\mathrm{~B}_{2} \mathrm{~B}_{6}$ & $1.888 \mathrm{~B}$ \\
$\mathrm{~B}_{4} \mathrm{~B}_{5}$ & $1.716 \mathrm{~B}$ \\
$\mathrm{~B}_{4} \mathrm{~B}_{0}$ & $1.698 \mathrm{~B}$ \\
$\mathrm{~B}_{2} \mathrm{C}$ & $1.599 \mathrm{~B}$ \\
$\mathrm{~B}_{4} \mathrm{C}$ & $1.633 \mathrm{~B}$ \\
\hline
\end{tabular}

The $C$ atom and each $B$ atom are bonded to an $H$ atom. The sevent? $\mathrm{H}$ atom was found to be a bridged atom in the $\mathrm{B}_{2} \mathrm{~B}_{3} \mathrm{~B}_{6}$ face.

[1] G. L. McKown, B. P. Don, R. A. Beaudet, P. J. Vergamini, and L. H. Jones, J. Amer. Chem. Soc. 98, 6909 (1976).

[2] G. L. McKown, B. P. Don, R. A. Beaudet, P. J. Vergamini, and L. H. Jones, J. Chem. Soc., Chem. Commun. 1974, 765 (1974).
Methylphosphine-borane

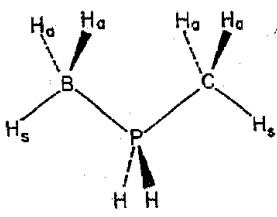

\begin{tabular}{|c|c|c|c|}
\hline Bond & Substitution & Angle & Substitution \\
\hline $\mathrm{CH}_{5}$ & $1.098 \mathrm{D}$ & $\mathrm{H}_{2} \mathrm{BH}_{3}$ & $116.1 \mathrm{C}$ \\
\hline $\mathrm{CH}_{3}$ & $1.087 \mathrm{~B}$ & $\mathrm{H}_{\Omega} \mathrm{BH}_{\mathrm{a}^{\prime}}$ & $112.3 \mathrm{C}$ \\
\hline $\mathrm{BH}_{\mathrm{a}}$ & $1.229 \mathrm{C}$ & $\mathrm{H}_{\Omega} \mathrm{CH}_{\mathrm{s}}$ & $110.4 \mathrm{D}$ \\
\hline $\mathrm{BH}_{\mathrm{s}}$ & $1.23 \mathrm{AC}$ & $\mathrm{H}_{3} \mathrm{CH}_{\mathrm{a}^{\prime}}$ & $108.1 \mathrm{~B}$ \\
\hline $\mathrm{PC}$ & $1.809 \mathrm{~B}$ & HPH' & $99.9 \mathrm{C}$ \\
\hline $\mathrm{PB}$ & $1.906 \mathrm{~B}$ & $\mathrm{HPC}$ & $103.2 \mathrm{C}$ \\
\hline \multirow[t]{5}{*}{ PH } & $1.404 \mathrm{~B}$ & $\begin{array}{l}\text { CPB } \\
\text { HPB }\end{array}$ & $\begin{array}{l}115.7 \mathrm{~B} \\
116.3 \mathrm{C}\end{array}$ \\
\hline & & $\mathrm{PBH}_{\mathrm{a}}$ & $102.9 \mathrm{C}$ \\
\hline & & $\mathrm{PBH}_{n}$ & $104.2 \mathrm{C}$ \\
\hline & & $\mathrm{PCH}_{\mathrm{a}}$ & $108.3 \mathrm{~B}$ \\
\hline & & $\mathrm{PCH}_{\mathrm{a}}$ & $111.3 \mathrm{D}$ \\
\hline
\end{tabular}

Subscripts a and $s$ refer to out-of-plane and in-plane atoms, respectively.

[1] P. S. Bryan and R. L. Kuczkowski, Inorg. Chem. 11, 553 (1972).

\section{2-Carbahexaborane}

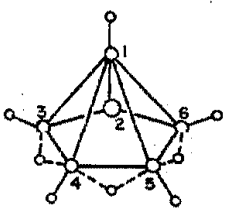

$\mathrm{CH}_{3} \mathrm{~B}_{0}$

\begin{tabular}{|c|c|c|c|}
\hline Bond & Substitution & Angle & Substitution \\
\hline $\mathrm{B}_{3} \mathrm{~B}_{4}$ & $1.759 \mathrm{~B}$ & $\mathrm{~B}_{3} \mathrm{~B}_{1} \mathrm{~B}_{4}$ & $59.11 \mathrm{C}$ \\
\hline$B_{4} B_{5}$ & $1.830 \mathrm{~B}$ & $B_{4} B_{1} B_{5}$ & $65.57 \mathrm{C}$ \\
\hline$B_{1} B_{3}$ & $1.782 \mathrm{~B}$ & $\mathrm{~B}_{3} \mathrm{~B}_{4} \mathrm{~B}_{3}$ & $103.9 \mathrm{C}$ \\
\hline$B_{1} B_{4}$ & $1.781 \mathrm{C}$ & & \\
\hline
\end{tabular}


1-Methylpentaborane (s)

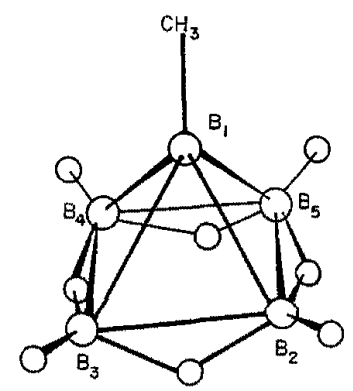

\begin{tabular}{ccc}
$\mathrm{CH}_{13} \mathrm{~B}_{5}$ & & $\mathrm{C}_{4 v}$ \\
\hline Bond & Substitution & Effective \\
\hline & & \\
$\mathrm{B}_{\mathrm{i}} \mathrm{B}_{2}$ & $1.687 \mathrm{C}$ & \\
$\mathrm{B}_{2} \mathrm{~B}_{3}$ & $1.800 \mathrm{C}$ & $1.62 \mathrm{X}$ \\
$\mathrm{B}_{1} \mathrm{C}$ & &
\end{tabular}

[1] E. A. Cohen and R. A. Beaudet, J. Chem. Phys. 48, 1220 (1968).

\section{Trimethylphosphine-borane}

\begin{tabular}{lcc|ccc}
$\mathrm{CH}_{12} \mathrm{BP}$ & & \multicolumn{2}{c}{$\left(\mathrm{CH}_{3}\right)_{3} \mathrm{P} \cdot \mathrm{BH}_{3}$} & $\mathrm{C}_{3 \mathrm{~F}}$ \\
\hline \hline Bond & $\begin{array}{c}\text { Substitu. } \\
\text { tion }\end{array}$ & Effective & Angle & $\begin{array}{c}\text { Substitu. } \\
\text { tion }\end{array}$ & Effective \\
\hline $\mathrm{PB}$ & & $1.901 \mathrm{D}$ & $\mathrm{CPC}$ & & $105.0 \mathrm{D}$ \\
$\mathrm{PC}$ & & $1.819 \mathrm{D}$ & $\mathrm{HBH}$ & $113.5 \mathrm{~B}$ & \\
$\mathrm{BH}$ & $1.212 \mathrm{C}$ & & & & \\
\hline
\end{tabular}

Methyl groups were assumed to be symmetrical and untilted, with $\mathrm{CH}=1.080$ and $\mathrm{HCH}=109.3$.

[1] P. S. Bryen and R. L. Kuczkowski, Inorg. Chem. 11, 553 (1972).

\section{Cyanogen iodide}

\begin{tabular}{cc} 
CIN & ICN \\
\hline \hline Bond & Substitution \\
\hline CI & $1.994 \mathrm{~B}$ \\
$\mathrm{CN}$ & $1.159 \mathrm{~A}$
\end{tabular}

[1] J. K. Tyler and J. Sheridan, Trans. Faraday Soc. 89, 2661 (1963).

J. Phys. Chem, Ref. Data, Vol. 8, No. 3, 1979

\section{Isocyanato}

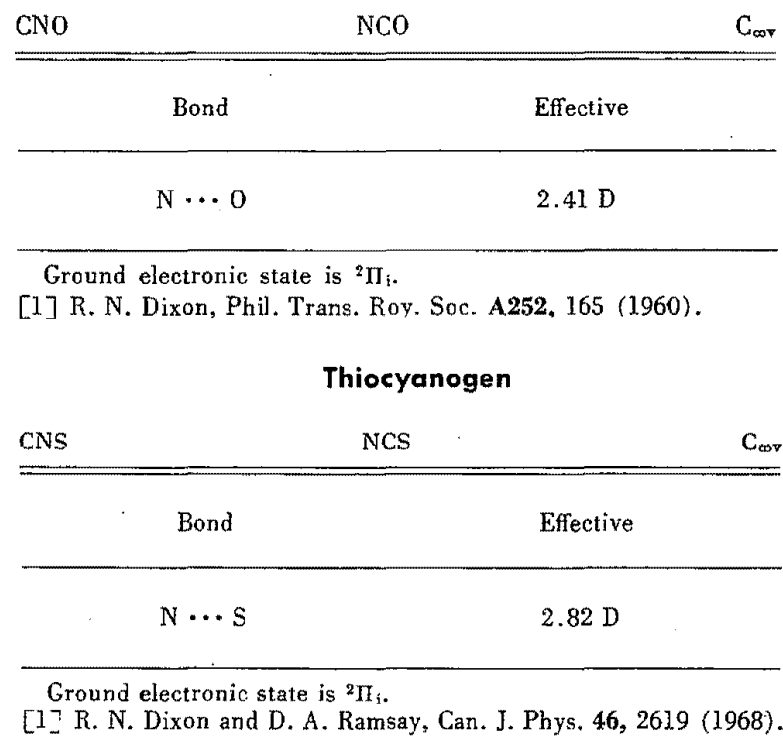

Cyanoimidogen

\begin{tabular}{lll}
$\mathrm{CN}_{2}$ & $\mathrm{NCN}$ & $\mathrm{D}_{\text {ob }}$ \\
\hline \hline Bond & Effective \\
\hline $\mathrm{CN}$ & $1.232 \mathrm{~A}$ \\
\hline
\end{tabular}

Ground electronic state is ${ }^{3} \Sigma_{\mathrm{q}}{ }^{-}$.

[1] G. Herzberg and D. N. Travis, Cans. J. Phys. 42, 1658 (1964).

Närrosyl eyanide

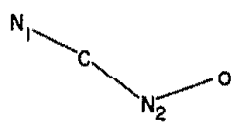

$\mathrm{CN}_{2} \mathrm{O}$

\begin{tabular}{ll|ll}
\hline \hline Bond & Effective & Angle & Effective \\
\hline & & & \\
$N_{1} \mathrm{C}$ & $1.170 \mathrm{C}$ & $\mathrm{NCN}$ & $172.5 \mathrm{E}$ \\
$\mathrm{CN}_{2}$ & $1.401 \mathrm{C}$ & $\mathrm{CNO}$ & $114.7 \mathrm{D}$ \\
$\mathrm{NO}$ & $1.228 \mathrm{C}$ & & \\
\hline
\end{tabular}

Conclusive evidence for planarity was not presented.

[l] R. Dickinson, G. W. Kirby, J. G. Sweeny and J. K. Tyler, J. Chem. Soc. Chem. Comm. 1973, 241 (1973). 


\section{Cyanogen Azide}

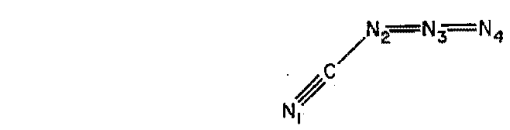

\begin{tabular}{lll|ll}
$\mathrm{CN}_{4}$ & & & $\mathrm{C}_{8}$ \\
\hline Bond & Substitution & Effective & Angle & Effective \\
\hline $\mathrm{CN}$ & $1.164 \mathrm{~B}$ & & & \\
$\mathrm{CN}_{2^{\mathrm{a}}}$ & & $1.312 \mathrm{D}$ & $\mathrm{CN}_{2} \mathrm{~N}_{3}^{\mathrm{a}}$ & $120.2 \mathrm{D}$ \\
$\mathrm{N}_{2} \mathrm{CN}_{2^{\mathrm{a}}}$ & $176 \mathrm{E}^{\mathrm{b}}$ \\
$\mathrm{N}_{3} \mathrm{~N}_{4^{\mathrm{at}}}^{\mathrm{a}}$ & & $1.252 \mathrm{D}$ & & \\
\hline
\end{tabular}

a Derived with assumptions that $\mathrm{N}_{2} \mathrm{~N}_{3} \mathrm{~N}_{4}$ linkage is linear and $\mathrm{N}_{3} \mathrm{~N}_{4}$ is $1.133 \pm 0.01 \mathrm{~A}$.

b $N_{1}$ bent away from $N_{3}$ linkage.

[1] K. Bolton, R. D. Brown and F. R. Burden, Chem. Phys. Lett. 15, 79 (1972).

[2] C. C. Costain and H. W. Kroto, Can. J. Phys. 50, 1453 (1972).

[3. G. L. Blackman, K. Bolton, K. D. Brown, K. K. Burden, and A. Mishra, J. Mol. Spentrosc. 47, 457 (1973).

\section{Carbonyl Sulfide}

\begin{tabular}{ccc} 
COS & OCS & $\mathrm{C}_{\infty}$ \\
\hline Bond & Equilibrium & Substitution \\
\hline CO & $1.157 \mathrm{~A}$ & $1.160 \mathrm{~B}$ \\
$\mathrm{CS}$ & $1.561 \mathrm{~A}$ & $1.560 \mathrm{~B}$
\end{tabular}

[1] Y. Morino and C. Matsumura, Bull. Chem. Soc. Japan 40, 1095 (1967).

[2] C. C. Costain, J. Chem. Phys, 29, 864 (1958).

\section{Carbonyl Selenide}

\begin{tabular}{ccc} 
CoSe & OCSe & $\mathrm{C}_{\text {our }}$ \\
\hline & Bond & Substitution \\
\hline & $\mathrm{Co}$ & $1.157 \mathrm{~B}$ \\
$\mathrm{CSe}$ & $1.708 \mathrm{~B}$
\end{tabular}

[1] Y. Morino and C. Matsumura, Bull. Chem. Soc. Japan 40, I101 (1967).

\section{Carbon Dioxide}

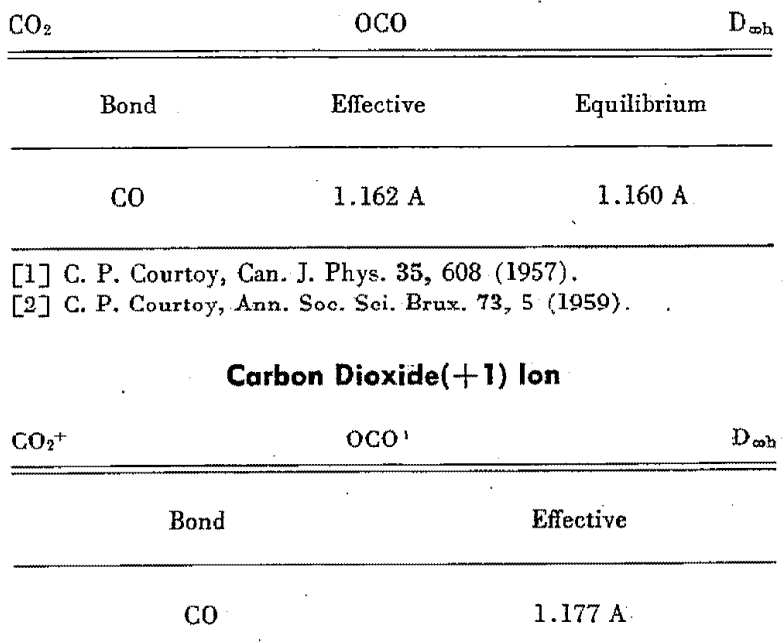

Crotand lectronic state is ${ }^{2} \pi$

[1] F. Bueso-Sanllehi, Phys. Rev. 60, 556 (1941).

[2] S. Mrozowski, Phys. Rev. 60, 730 (1941).

[3] S. Mrozowski, Phys. Rev. 62, 270 (1942).

[4] S. Mrozowski. Phys: Rey. 72. 682. 691 (1947).

\section{Carbon Sulfide Selenide}

\begin{tabular}{lcc} 
CSSe & SCSe & $\mathrm{C}_{\text {orv }}$ \\
\hline \hline Bond & Effective \\
\hline & & $1.553 \mathrm{~A}$ \\
$\mathrm{CS}$ & $1.695 \mathrm{~A}$
\end{tabular}

[1] C. Hirose and R. F. Curl, Jr., J. Chem. Phys. 55, 5120 (197]).

Carbon Sulfide Telluride

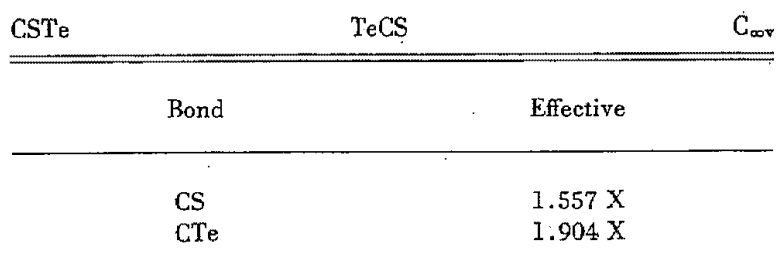

[1] W. A. Hardy and G. Silvey, Phys. Rev. 95, 385 (1954). 


\section{Carbon Disulfide}

\begin{tabular}{ccc}
$\mathrm{CS}_{3}$ & $\mathrm{SCS}$ & $\mathrm{D}_{\infty \mathrm{s}}$ \\
\hline \hline Bond & Effective & Equilibrium \\
\hline CS & $1.554 \mathrm{~A}$ & $1.553 \mathrm{~A}$
\end{tabular}

[1] G. Blanquet, J. Walrand and C. P. Courtoy, Ann. Soc. Sci. Brux. 88, 87 (1974).

[2] A. G. Maki and R. L. Sams, J. Mol. Spectrose. 52, 233 (1974).

Carbon Disulfide $(+1)$ Ion

\begin{tabular}{ccc}
$\mathrm{CS}_{2}{ }^{+}$ & $\mathrm{SCS}^{+}$ & $D_{\infty \mathrm{h}}$ \\
\hline Bond & Effective \\
\hline $\mathrm{CS}$ & $1.554 \mathrm{~A}$ \\
\hline
\end{tabular}

Ground electronic state is ${ }^{2} \mathrm{II}_{\mathrm{g}}$.

[1] J. H. Callomon, Proc. Roy. Soc. A244, 220 (1958).

\section{$\mathrm{C}_{2}$ Molecules}

\section{Chloroiodoacefylene}

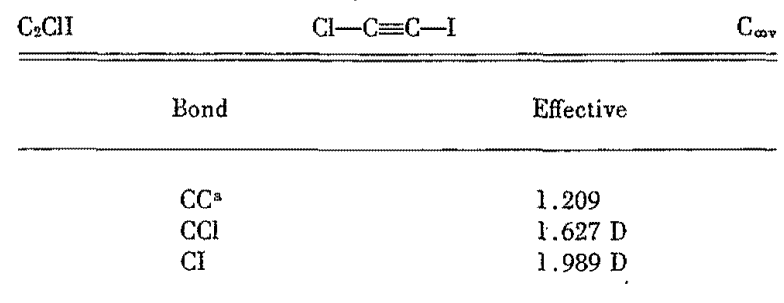

Assumed value.

[1] A. Bjorseth, E. Kloster-Jensen, K. M. Marstokk and H. Mollendal, J. Mol. Struct. 6, 181 (1970).

\section{1,1,1-Trifluoro-2,2,2-trichloroethane}

\begin{tabular}{cc|cc}
$\mathrm{C}_{2} \mathrm{Cl}_{3} \mathrm{~F}_{3}$ & \multicolumn{2}{c}{$\mathrm{F}_{3} \mathrm{C}-\mathrm{CCl}_{3}$} & $\mathrm{C}_{3 \%}$ \\
\hline Bond & Effective & Angle & Effective \\
\hline $\mathrm{CC}$ & $1.539 \times$ & $\mathrm{CCF}$ & $109.6 \mathrm{X}$ \\
$\mathrm{CF}$ & $1.330 \mathrm{X}$ & $\mathrm{CCCl}$ & $109.6 \mathrm{X}$ \\
$\mathrm{CCl}$ & $1.771 \mathrm{X}$ & & \\
\hline
\end{tabular}

[1] R. Holm, M. Mitzlaff and H. Hartmann, Z. Naturforsch. 23a, 1040 (1968).

\section{Trichloroacetonitrile}

\begin{tabular}{cc|cc}
$\mathrm{CrCl}_{3} \mathrm{~N}$ & $\mathrm{Cl}_{3} \mathrm{CCN}$ & $\mathrm{C}_{38}$ \\
\hline Bond & Effective & Angle & Effective \\
\hline $\mathrm{CCl}$ & $1.771 \mathrm{E}$ & $\mathrm{CCCl}$ & $108.9 \mathrm{E}$ \\
\hline
\end{tabular}

$\mathrm{CN}$ and $\mathrm{CC}$ bond lengths assumed.

[I] A. S. Rajan, Proc. Indian Aced. Sci. 53, 89 (1961).

\section{Trifluoraacefonitrile}

\begin{tabular}{cc|cc}
$\mathrm{C}_{2} \mathrm{~F}_{3} \mathrm{~N}$ & $\mathrm{CF}_{3} \mathrm{CN}$ & $\mathrm{C}_{34}$ \\
\hline \hline Bond & Effective & Angle & Effective \\
\hline $\mathrm{CN}$ & $1.158^{\mathrm{a}}$ & $\mathrm{FCF}$ & $108.0^{\mathrm{a}}$ \\
$\mathrm{CF}$ & $1.335 \mathrm{X}$ & & \\
$\mathrm{CC}$ & $1.464 \mathrm{X}$ & & \\
\hline
\end{tabular}

a Assumed parameters.

[1] J. Sheridan and W. Gordy, J. Chem. Phys. 20, 591 (1952).

\section{Ethynyldifluoroborane}

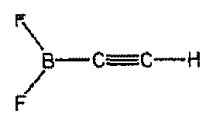

$\mathrm{C}_{2} \mathrm{HBF}_{2}$

$\mathrm{C}_{24}$

\begin{tabular}{ll|ll}
\hline Bond & Substitution & Angle & Substitution \\
\hline $\mathrm{CC}$ & $1.206 \mathrm{~A}$ & FBF & $116.5 \mathrm{C}$ \\
$\mathrm{CB}$ & $1.513 \mathrm{~B}$ & & \\
$\mathrm{CH}$ & $1.058 \mathrm{~B}$ & & \\
$\mathrm{BF}$ & $1.323 \mathrm{C}$ & & \\
\hline
\end{tabular}

The fiworine parameters are not strictly substitution, since one moment of inertia relation was solved directly to obtain $x$ coordinates.

[1] W. J. Lafferty and J. J. Ritter, J. Mol. Spectrosc. 38, 181 (1971).

[2] W. J. Lafferty and J. J. Ritter, Chem. Comm. 1969, 909 (1969).

\begin{tabular}{ccc}
\multicolumn{3}{c}{ Bromoacetylene } \\
$\mathrm{C}_{2} \mathrm{HBr}$ & $\mathrm{HC}=\mathrm{CBr}$ & $\mathrm{C}_{\text {orr }}$ \\
\hline \hline Bond & Substitution & Effective \\
\hline $\mathrm{CH}$ & & $1.051 \mathrm{C}$ \\
$\mathrm{CC}$ & & $1.216 \mathrm{C}$ \\
$\mathrm{CBr}$ & $1.784 \mathrm{C}$ \\
$\mathrm{H} \cdots \mathrm{Br}$ & $4.051 \mathrm{~A}$ &
\end{tabular}

[1] H. Jones, N. L. Owen, J. Sheridan, Nature 213, 175 (1967). 


\section{Chloroacetylene}

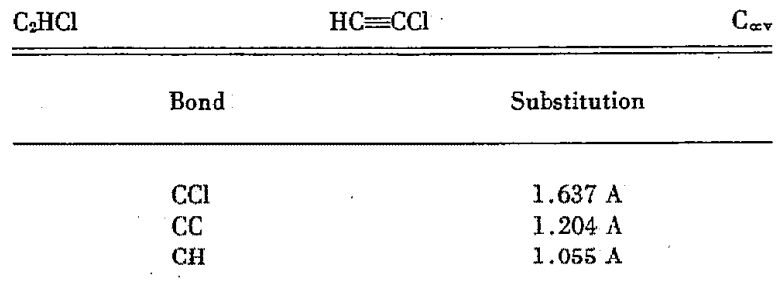

[1] J. K. Tyler and J. Sheridan, Trans, Faraday Soc. 89, 2661 (1963).

\section{Fluoroacelylene}

\begin{tabular}{|c|c|}
\hline Bond & Substitution \\
\hline CF & $1.279 \mathrm{~B}$ \\
\hline CC & $1.198 \mathrm{~B}$ \\
\hline $\mathrm{CH}$ & $1.053 \mathrm{~B}$ \\
\hline
\end{tabular}

Authors checked $\mathrm{C}_{1}$ coordinate by double-substitution method since atom is close to C. of M. Agreement is well within experimental limits.

[1] J. K. Tyler and J. Sheridan, Trans. Faraday Soc. 89, 2601 (1963).

[2] J. K. Tyler and J. Sheridan, Proc. Chem. Soc. 1960, 119.

\section{lodoacetylene}

$\mathrm{C}_{2} \mathrm{HI}$

$\mathrm{HC} \equiv \mathrm{CI}$

$\mathrm{C}_{\infty \mathrm{r}}$

\begin{tabular}{cc}
\hline Bond & Effective \\
\hline $\mathrm{CI}$ & $1.988 \mathrm{X}$
\end{tabular}

In order to obtain the C-I distance it was necessary to assume both the $\mathrm{C} \equiv \mathrm{C}$ and $\mathrm{C}-\mathrm{H}$ bond distances. The unknown uncertainty in the assumptions makes it difficult to gauge the uncertainty in in the $\mathrm{CI}$ distance.

[1] W. Jeremy Jones, B. P. Stoicheff and J. K. Tyler, Can. J. Phys. 41, 2098 (1963).
Diazoacefonitrile

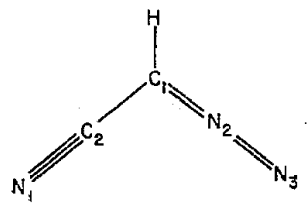

$\mathrm{C}_{2} \mathrm{HN}_{3}$

\begin{tabular}{lrr|ll}
\hline \hline Bond & Substitution & Effective & Angle & Effective \\
\hline & & $1.165 \mathrm{X}$ & $\mathrm{C}_{2} \mathrm{C}_{2} \mathrm{~N}_{2}$ & $119.5 \mathrm{X}$ \\
$\mathrm{C}_{2} \mathrm{~N}_{1}$ & & $1.424 \mathrm{X}$ & $\mathrm{HC}_{2} \mathrm{C}_{2}$ & $117 \mathrm{X}$ \\
$\mathrm{C}_{2} \mathrm{C}_{1}$ & & & \\
$\mathrm{C}_{1} \mathrm{~N}_{2}$ & $1.280 \mathrm{C}$ & & \\
$\mathrm{N}_{2} \mathrm{~N}_{3}$ & & $1.132 \mathrm{X}$ & & \\
\hline
\end{tabular}

Linearity of $\mathrm{C}_{1} \mathrm{~N}_{2} \mathrm{~N}_{3}$ was assumed. $\mathrm{CH}$ bond assumed.

[1] C. C. Costain and J. Yarwrood, J. Chem. Phys. 45, 1961 (1965).

\begin{tabular}{|c|c|c|}
\hline \multirow[b]{2}{*}{$\mathrm{C}_{2} \mathrm{H}_{2}$} & \multicolumn{2}{|l|}{ Acetylene } \\
\hline & $\mathrm{HC}=\mathrm{CH}$ & $\mathrm{D}_{\operatorname{\infty in}}$ \\
\hline Bond & Substitution & Equilibrium \\
\hline cII & $1.050 \mathrm{~B}$ & $1.061 \AA$ \\
\hline $\mathrm{CC}$ & $1.207 \mathrm{C}$ & $1.203 \mathrm{~A}$ \\
\hline
\end{tabular}

[1] W. J. Lafferty and R. J. Thibault, J. Mol. Spectrose. 14,. 79 (1964).

[2] T. A. Wiggins, E. K. Plyler and E. D. Tidwell, J. Opt. Soc. Am. 51, 1219 (1961)

[3] E. K. Plylex, E. D. Tidwell and T. A. Wiggins, J. Opt. Soc. Am. 53,589 (1963).

[4] W. J. Lafferty, E. K. Plyler and E. D. Tidwell, J. Chem. Phys. 37,1981 (1962).

[5] E. D. Tidwell and E. K. Plyler, J. Opt. Soc. Am. 52, 656 (1962).

\section{Bromoacetonitrile}

\begin{tabular}{cc|cc}
$\mathrm{C}_{2} \mathrm{H}_{2} \mathrm{BrN}$ & & $\mathrm{BrCH}_{2} \mathrm{CN}$ \\
\hline \hline Bond & Effective & Angle & Effective \\
\hline & & & $111.5 \mathrm{X}$ \\
$\mathrm{CN}$ & $1.158 \mathrm{X}$ & $\mathrm{CBr}$ & $102.9 \mathrm{X}$ \\
$\mathrm{CBr}$ & $1.901 \mathrm{X}$ & $\mathrm{CCH}$ & $180 \mathrm{X}$ \\
$\mathrm{CH}$ & $1.107 \mathrm{X}$ & $\mathrm{CCN}$ & \\
$\mathrm{CC}$ & $1.487 \mathrm{X}$ & &
\end{tabular}

[1] M. L. Gum and J. D. Graybeal, J. Mol. Spectrosc. 62, 364 (1976). 
I-Chloro-1-fluoroethylene

\begin{tabular}{cc|cc}
$\mathrm{C}_{2} \mathrm{H}_{2} \mathrm{ClF}$ & $\mathrm{H}_{2} \mathrm{C}=\mathrm{CClF}$ & $\mathrm{C}_{\mathrm{B}}$ \\
\hline Bond & Effective & Angle & Effective \\
\hline & & & \\
$\mathrm{CC}$ & $1.315 \mathrm{X}$ & $\mathrm{CCH}$ & $120.0 \mathrm{X}$ \\
$\mathrm{CH}$ & $1.079 \mathrm{X}$ & $\mathrm{CCF}$ & $123.9 \mathrm{X}$ \\
$\mathrm{CF}$ & $1.325 \mathrm{X}$ & $\mathrm{CCCl}$ & $124.5 \mathrm{X}$ \\
$\mathrm{CCl}$ & $1.725 \mathrm{X}$ & & \\
\end{tabular}

[1] R. G. Stone and W. H. Flygare, J. Mol. Spectrosc. 32, 233 (1969).

\section{Chloroacetonitrile}

\begin{tabular}{cc|cc}
$\mathrm{C}_{2} \mathrm{H}_{2} \mathrm{ClN}$ & $\mathrm{C}^{1} \mathrm{H}_{2} \mathrm{ClC}^{2} \mathrm{~N}$ & $\mathrm{C}_{8}$ \\
\hline \hline Bond & Substitution & Angle & Substitution \\
\hline $\mathrm{C}^{\mathrm{C} C l}$ & $1.781 \mathrm{X}$ & $\mathrm{C}^{2} \mathrm{C}^{1} \mathrm{Cl}$ & $111.5 \mathrm{X}$ \\
$\mathrm{C}^{1} \mathrm{H}$ & $1.088 \mathrm{X}$ & $\mathrm{C}^{2} \mathrm{C}^{1} \mathrm{H}$ & $107.5 \mathrm{X}$ \\
\hline
\end{tabular}

$\mathrm{C}^{2} \mathrm{C}^{2}$ and $\mathrm{C}^{2 \mathrm{~N}}$ bond lengthe acoumed. $\mathrm{C}^{1} \mathrm{C}^{2}$ assumed linear.

[1] K. Wada, Y. Kikuchi, C. Matsumura, E. Hirota, and Y. Morino, Bull. Chem. Soc. Japan 34, 33 ? (1961).

\section{1,1-Dinuorothylene}

\begin{tabular}{lcc|ccc}
$\mathrm{C}_{2} \mathrm{H}_{2} \mathrm{~F}_{2}$ & & \multicolumn{3}{c}{$\mathrm{H}_{2} \mathrm{C}=\mathrm{CF}_{2}$} & $\mathrm{C}_{2 \mathrm{v}}$ \\
\hline \hline Bond & $\begin{array}{c}\text { Substitu- } \\
\text { tion }\end{array}$ & Effective & Angle & $\begin{array}{c}\text { Substitu- } \\
\text { tion }\end{array}$ & Effective \\
\hline $\mathrm{CC}$ & & $1.315 \mathrm{D}$ & $\mathrm{HCH}$ & $121.1 \mathrm{C}$ & \\
$\mathrm{CH}$ & $1.074 \mathrm{C}$ & & $\mathrm{FCF}$ & & $109.0 \mathrm{D}$ \\
$\mathrm{CF}$ & & $1.323 \mathrm{D}$ & & & \\
\hline
\end{tabular}

[I] V. W. Laurie and D. T. Pence, J. Chem. Phys. 38, 2693 (1963).

\section{cis 1,-2-Difluoroethylene}

\begin{tabular}{lcc|ccc}
$\mathrm{C}_{2} \mathrm{H}_{2} \mathrm{~F}_{2}$ & & $\mathrm{HFC}=\mathrm{CHF}$ & $\mathrm{C}_{2 \mathrm{~V}}$ \\
\hline Bond & $\begin{array}{c}\text { Substitu- } \\
\text { tion }\end{array}$ & Effective & Angle & $\begin{array}{c}\text { Substitu- } \\
\text { tion }\end{array}$ & Effective \\
\hline $\mathrm{CC}$ & $1.325 \mathrm{~A}$ & & $\mathrm{FCC}$ & & $122.1 \mathrm{~A}$ \\
$\mathrm{CH}$ & $1.088 \mathrm{~A}$ & & $\mathrm{HCC}$ & $123.9 \mathrm{~A}$ & \\
$\mathrm{CF}$ & & $1.337 \mathrm{~A}$ & & & \\
\hline
\end{tabular}

[1] V. W. Laurie and D. T. Pence, J. Chem. Phys. 39, 2693 (1963)

\section{trans-Fluoroacetyl fluoride}

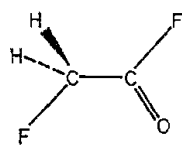

$\mathrm{C}_{2} \mathrm{H}_{2} \mathrm{~F}_{2} \mathrm{O}$

$\mathrm{C}_{\mathrm{s}}$

No detailed structural information is available. A less stable $\left(910 \mathrm{cal} \mathrm{mol}^{-1}\right)$ cis rotamer was also definitely identified.

[1] E. Saegebarth and E. B. Wilson, Jr., J. Chem. Phys. 46, 3088 (1967).

\section{Hydrogen cyanide dimer}

$\mathrm{C}_{2} \mathrm{H}_{2} \mathrm{~N}_{2}$

$\mathrm{HCN} \cdots \mathrm{HCN}$

$\mathrm{C}_{\text {oov }}$

Assuming unchanged monomer geometry, the $\mathrm{N}$... C distance is reported to be $3.23 \AA$.

[1] A. C. Legon, D. J. Millen and P. J. Mjoberg, Chem. Phys. Lett. $47,589(1977)$.

\section{1,2,5-Oxadiazole}

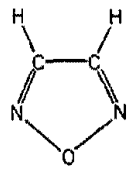

\begin{tabular}{cc|cc}
$\mathrm{C}_{2} \mathrm{H}_{2} \mathrm{~N}_{2} \mathrm{O}$ & & \\
\hline \hline Bond & Substitution & Angle & Substitution \\
\hline & & & \\
$\mathrm{NO}$ & $1.380 \mathrm{C}$ & $\mathrm{NON}$ & $110.4 \mathrm{C}$ \\
$\mathrm{CN}$ & $1.300 \mathrm{~B}$ & $\mathrm{ONC}$ & $105.8 \mathrm{~B}$ \\
$\mathrm{CC}$ & $1.421 \mathrm{~B}$ & $\mathrm{CCN}$ & $109.0 \mathrm{~A}$ \\
$\mathrm{CH}$ & $1.076 \mathrm{~B}$ & $\mathrm{CCH}$ & $130.2 \mathrm{~A}$ \\
& & &
\end{tabular}

[1] E. Saegebarth and A. P. Cox, J. Chem. Phys. 43, 166 (1965). 


\section{1,3,4-Oxadiazole}

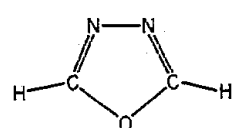

$\mathrm{C}_{2} \mathrm{H}_{2} \mathrm{~N}_{2} \mathrm{O}$

\begin{tabular}{ll|ll}
\hline Bond & Substitution & Angle & Substitution \\
\hline & & & \\
CO & $1.348 \mathrm{~B}$ & $\mathrm{COC}$ & $102.0 \mathrm{C}$ \\
$\mathrm{CN}$ & $1.297 \mathrm{C}$ & $\mathrm{OCN}$ & $113.4 \mathrm{~B}$ \\
$\mathrm{NN}$ & $1.399 \mathrm{~B}$ & $\mathrm{CNN}$ & $105.6 \mathrm{~B}$ \\
$\mathrm{CH}$ & $1.075 \mathrm{~B}$ & $\mathrm{OCH}$ & $118.1 \mathrm{~B}$ \\
& & $\mathrm{NCH}$ & $128.5 \mathrm{~B}$ \\
\hline
\end{tabular}

[I] L. Nygaard, R. L. Hansen, J. T. Nielsen, J. Rastrup-Andersen, G. O. Sorensen and P. A. Steiner, J. Mol. Struct. 12, 59 (1972).

\section{1,2,4-Thiadiazole}

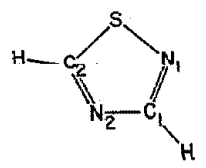

\begin{tabular}{ll|ll}
$\mathrm{C}_{2} \mathrm{H}_{2} \mathrm{~N}_{2} \mathrm{~S}$ & & & $\mathrm{C}_{2}$ \\
\hline \hline Bond & Substitution & Angle & Substitution \\
\hline & & & \\
\hline & & & \\
$\mathrm{SN}_{1}$ & $1.649 \mathrm{~A}$ & $\mathrm{C}_{2} \mathrm{SN}_{1}$ & $92.8 \mathrm{~A}$ \\
$\mathrm{~N}_{1} \mathrm{C}_{1}$ & $1.317 \mathrm{~A}$ & $\mathrm{SN}_{1} \mathrm{C}_{1}$ & $107.1 \mathrm{~A}$ \\
$\mathrm{C}_{1} \mathrm{~N}_{2}$ & $1.366 \mathrm{~A}$ & $\mathrm{~N}_{1} \mathrm{C}_{1} \mathrm{~N}_{2}$ & $120.1 \mathrm{~A}$ \\
$\mathrm{~N}_{2} \mathrm{C}_{2}$ & $1.313 \mathrm{~A}$ & $\mathrm{C}_{1} \mathrm{~N}_{2} \mathrm{C}_{2}$ & $107.7 \mathrm{~A}$ \\
$\mathrm{C}_{2} \mathrm{~S}$ & $1.707 \mathrm{~A}$ & $\mathrm{~N}_{2} \mathrm{C}_{2} \mathrm{~S}$ & $112.3 \mathrm{~A}$ \\
$\mathrm{C}_{2} \mathrm{H}$ & $1.078 \mathrm{~B}$ & $\mathrm{SC}_{2} \mathrm{H}$ & $123.9 \mathrm{~B}$ \\
$\mathrm{C}_{1} \mathrm{H}$ & 1.078 (Assumed) & $\mathrm{N}_{2} \mathrm{C}_{1} \mathrm{H}$ & 119.9 (Assumed)
\end{tabular}

Small coordinates were present, but abundance of isotopic data yields high reliability to the otruotural paramotcrs.

[l] O. Li. Steifvater, Z. Naturforsch, 31a, 1681 (1976).

\section{1,2,5-Thiadiazole}

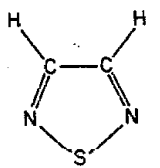

\begin{tabular}{|c|c|c|c|c|c|}
\hline Bond & $\begin{array}{l}\text { Substitu- } \\
\text { tion }\end{array}$ & Effective & Angle & $\begin{array}{c}\text { Substitu. } \\
\text { tion }\end{array}$ & Effective \\
\hline SN & & $1.631 \mathrm{~B}$ & NSN & & $99.5 \mathrm{~B}$ \\
\hline $\mathrm{NC}$ & & $1.328 \mathrm{~B}$ & CNS & & $106.4 \mathrm{~B}$ \\
\hline $\mathrm{CC}$ & $1.418 \mathrm{~B}$ & & $\mathrm{CCN}$ & & $113: 8 \mathrm{~B}$ \\
\hline $\mathrm{CH}$ & & $1.079 \mathrm{~B}$ & $\mathrm{CCH}$ & $126.2 \mathrm{~B}$ & \\
\hline
\end{tabular}

[I] Sr. V. Dobyns and L. Pierce, J. Amer. Chem. Soc. 85, 3553 (1963).

1,3,4-Thiadiarole

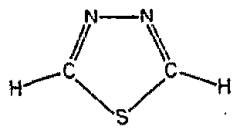

$\mathrm{C}_{2} \mathrm{H}_{2} \mathrm{~N}_{2} \mathrm{~S}$

$\mathrm{C}_{8 \mathrm{r}}$

\begin{tabular}{ll|ll}
\hline \hline Bond & Substitution & Angle & Substitution \\
\hline & & & \\
SC & $1.721 \mathrm{~B}$ & CSC & $86.4 \mathrm{~A}$ \\
CN & $1.302 \mathrm{~B}$ & SCN & $114.6 \mathrm{~A}$ \\
NN & $1.371 \mathrm{~B}$ & $\mathrm{CNN}$ & $112.2 \mathrm{~A}$ \\
CH & $1.079 \mathrm{~B}$ & SCH & $121.9 \mathrm{C}$ \\
& & NCH & $123.5 \mathrm{C}$
\end{tabular}

[1] L. Nygaard, R. Lykke Hansen, and G. O. Sorensen, J. Mol. Struct. 9, 163 (1971).

[2] B. Bak, L. Nygaard, E. J. Pedersen, and J. Rastrup-Andersen, J. Mol. Spectrosc. 19, 283 (1966).

\section{1,2,5-Selenadiazole}

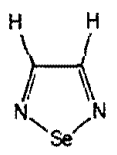

$\mathrm{C}_{2} \mathrm{H}_{2} \mathrm{~N}_{2} \mathrm{Se}$

Planar molecule with $C_{2 v}$ symmetry established.

[I] G. L. Blackman, R. D. Brown, F. R. Burden, and J. E. Kent, Chem. Phys. Lett. 1, 379 (1967). 


\section{1,3,4-Selenadiazole}<smiles>[C+]1=NN=C[Se]1</smiles>

$\mathrm{C}_{2} \mathrm{H}_{2} \mathrm{~N}_{2} \mathrm{Se}$

A planar molecule with $\mathrm{C}_{2 \pi}$ symmetry is definitely established.

[1] D. M. Levine, W. D. Krugh, and L. P. Gold, J. Mol. Spectrosc. 30, 459 (1969).

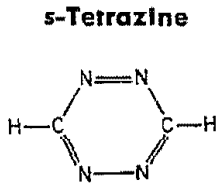

$\mathrm{C}_{2} \mathrm{H}_{2} \mathrm{~N}_{4}$

\begin{tabular}{ll|lc}
\hline Bond & Substitution & Angle & Substitution \\
\hline $\mathrm{CH}$ & $1.07 \mathrm{D}$ & $\mathrm{NCN}$ & $124.6 \mathrm{D}$ \\
$\mathrm{CN}$ & $1.338 \mathrm{D}$ & & \\
$\mathrm{NN}$ & $1.330 \mathrm{D}$ & &
\end{tabular}

[1] A. J. Merer and K. K. Innes, Proc. Roy. Soc. (London) A302, $271(1968)$.

Kotono

\begin{tabular}{|c|c|c|c|c|}
\hline $\mathrm{C}_{2} \mathrm{H}_{2} \mathrm{O}$ & & $\mathrm{H}_{2} \mathrm{C}=\mathrm{C}=$ & & \\
\hline Bond & Substitution & Effective & Angle & Effective \\
\hline $\mathrm{CO}$ & $1.161 \mathrm{~B}$ & \multirow[b]{3}{*}{$1.077 \mathrm{~B}$} & \multirow[t]{3}{*}{$\mathrm{HCH}$} & \multirow[t]{3}{*}{$122.2 \mathrm{~B}$} \\
\hline $\mathrm{CC}$ & $1.314 \mathrm{~B}$ & & & \\
\hline $\mathrm{CH}$ & & & & \\
\hline
\end{tabular}

[1] J. W. C. Johne, J. M. R. Stone and C. Winnetviecer, J. Mol Spectrosc. 42, 523 (1972).

[2] A. P. Cox, L. F. Thomas and J. Sheridan, Spectrochim. Acta 15, 542 (1959)

[3] I. Sheridan, Molecular Spectroscopy Pergamon Press, New York 1959 , pp. 139-147.

[4.] H. R. Johnson and M. W. P. Strandberg, J. Chem. Phys. 20, 687 (1952).
cis-Glyoxal

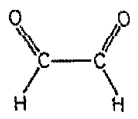

\begin{tabular}{cc|cc}
$\mathrm{C}_{2} \mathrm{H}_{2} \mathrm{O}_{2}$ & & \\
\hline Bond & Effective & Angle & Effective \\
\hline & & & \\
\hline $\mathrm{CC}$ & $1.514 \mathrm{X}$ & $\mathrm{CCH}$ & $116.2 \mathrm{X}$ \\
$\mathrm{CH}$ & $1.130 \mathrm{X}$ & $\mathrm{CCO}$ & $123.4 \mathrm{D}$
\end{tabular}.

CO distance assumed (1.207)

[1] A. R. H. Cole, Y. S. Li and J. R. Durig, J. Mol. Spectrosc. 61, 346 (1976).

[2] D. A. Ramsey and C. Zauli, Acta Plys. Acad. Sci. Ifungaricac $35,79(1974)$.

\section{trans- Clyoxal}

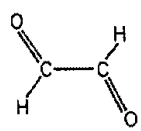

\begin{tabular}{cc|cc}
$\mathrm{C}_{2} \mathrm{H}_{2} \mathrm{O}_{2}$ & & $\mathrm{C}_{2 \mathrm{~b}}$ \\
\hline Bond & Effective & Angle & Effective \\
& & & \\
\hline $\mathrm{CH}$ & $1.109 \mathrm{C}$ & $\mathrm{CCH}$ & $115.5 \mathrm{E}$ \\
$\mathrm{CC}$ & $1.527 \mathrm{D}$ & $\mathrm{CCO}$ & $121.1 \mathrm{~A}$ \\
$\mathrm{CO}$ & $1.202 \mathrm{D}$ & & \\
\hline
\end{tabular}

[1] F. W. Birss, D. B. Braund, A. R. H. Cole, R. Engleman, Jr., A. A. Green, S. M. Japar, R. Nanes, B. J. Orr, D. A. Ramsay, and J. Szyszka, Can. J. Phys. 55, 390 (1977).

\section{Formle anhydride}

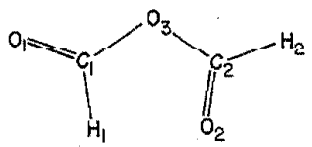

$\mathrm{C}_{2} \mathrm{H}_{2} \mathrm{O}_{3}$

\begin{tabular}{cc|cc}
\hline Bond & Substitution & Angle & Substitution \\
\hline $\mathrm{C}_{1} \mathrm{O}_{1}$ & $1.184 \mathrm{~B}$ & $\mathrm{C}_{1} \mathrm{C}_{1} \mathrm{O}_{3}$ & $120.6 \mathrm{~B}$ \\
$\mathrm{C}_{1} \mathrm{H}_{1}$ & $1.101 \mathrm{~B}$ & $\mathrm{H}_{1} \mathrm{C}_{1} \mathrm{O}_{3}$ & $112.1 \mathrm{~B}$ \\
$\mathrm{C}_{1} \mathrm{O}_{3}$ & $1.389 \mathrm{~B}$ & $\mathrm{C}_{1} \mathrm{O}_{3} \mathrm{C}_{2}$ & $117.8 \mathrm{~B}$ \\
$\mathrm{C}_{2} \mathrm{O}_{3}$ & $1.364 \mathrm{~A}$ & $\mathrm{H}_{2} \mathrm{C}_{2} \mathrm{O}_{3}$ & $108.6 \mathrm{~B}$ \\
$\mathrm{C}_{2} \mathrm{O}_{2}$ & $1.195 \mathrm{~B}$ & $\mathrm{O}_{2} \mathrm{C}_{2} \mathrm{O}_{3}$ & $126.1 \mathrm{~B}$ \\
$\mathrm{C}_{2} \mathrm{H}_{2}$ & $1.096 \mathrm{~B}$ & & \\
\hline
\end{tabular}

[1] S. Vaccani, U. Roos, A. Bauder and Hs. H. Günthard, Chem. Phys. 19, 51 (1977). 
Glyoxylic acid

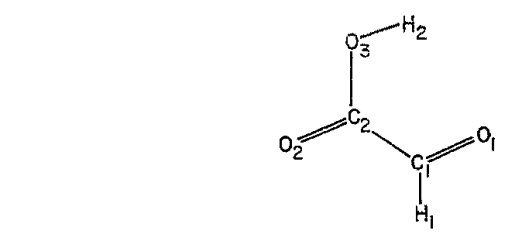

$\mathrm{C}_{2} \mathrm{H}_{2} \mathrm{O}_{3}$

\begin{tabular}{ll|ll}
\hline \hline Bond & Substitution & Angle & Substitution \\
\hline & & & \\
$\mathrm{C}_{1} \mathrm{O}_{1}$ & $1.174 \mathrm{C}$ & $\mathrm{C}_{2} \mathrm{C}_{1} \mathrm{O}_{1}$ & $123.7 \mathrm{C}$ \\
$\mathrm{C}_{2} \mathrm{O}_{2}$ & $1.203 \mathrm{C}$ & $\mathrm{C}_{1} \mathrm{C}_{2} \mathrm{O}_{2}$ & $121.3 \mathrm{C}$ \\
$\mathrm{C}_{2} \mathrm{O}_{3}$ & $1.313 \mathrm{D}$ & $\mathrm{O}_{2} \mathrm{C}_{2} \mathrm{O}_{3}$ & $126.2 \mathrm{C}$ \\
$\mathrm{C}_{1} \mathrm{C}_{2}$ & $1.535 \mathrm{C}$ & $\mathrm{COH}$ & $107.8 \mathrm{~B}$ \\
$\mathrm{CH}$ & $1.104 \mathrm{D}$ & $\mathrm{HCO}$ & $121.7 \mathrm{C}$ \\
$\mathrm{OH}$ & $0.948 \mathrm{~B}$ & $\mathrm{HCC}$ & $114.6 \mathrm{D}$ \\
& & $\mathrm{C}_{1} \mathrm{C}_{2} \mathrm{O}_{3}$ & $112.5 \mathrm{C}$
\end{tabular}

[1] I. Christiansen, K. M. Marstokk and H. Mollendal, J. Mol. Struct. 30, 137 (1976).

[2] K. M. Marstokk and H. Mollendal, J. Mol. Struct. 15, 137 (1973).

\section{Bromoethylene (Vinyl Bromide)}

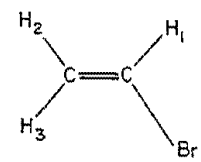

$\mathrm{C}_{8} \mathrm{H}_{6} \mathrm{Br}$

\begin{tabular}{lc|ll}
\hline \hline Bond & Substitution & Angle & Substitution \\
\hline & & & \\
$\mathrm{CBr}$ & $1.904 . \mathrm{D}$ & $\mathrm{CCBr}$ & $120.7 \mathrm{D}$ \\
$\mathrm{CC}$ & $1.334 . \mathrm{B}$ & $\mathrm{CCH}_{1}$ & $124.3 \mathrm{~B}$ \\
$\mathrm{CH}_{1}$ & $1.074 \mathrm{~B}$ & $\mathrm{CCH}_{2}$ & $121.0 \mathrm{X}$ \\
$\mathrm{CH}_{2}$ & $1.070 \mathrm{X}$ & $\mathrm{CCH}_{2}$ & $121.7 \mathrm{~B}$ \\
$\mathrm{CH}_{3}$ & $1.084 \mathrm{C}$ & & \\
\hline
\end{tabular}

[1] R. E. Goedertier, J. Physique 24, 633 (1963).

3-Mefhyl-3-bromodiazirine

\begin{tabular}{ccc}
$\mathrm{C}_{2} \mathrm{H}_{3} \mathrm{BrN}_{2}$ & $\mathrm{Br}\left(\mathrm{CH}_{3}\right) \mathrm{C}-\mathrm{N}=\mathrm{N}$ & $\mathrm{C}_{5}$ \\
\hline Bond & Efrecuive \\
\hline $\mathrm{NN}$ & $1.240 \mathrm{C}$
\end{tabular}

[I] J. E. Wollrab, J. Chem. Phys. 53, 1543 (1970).

\section{Acefyl bromide}

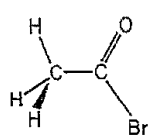

\begin{tabular}{cc|cr}
$\mathrm{C}_{2} \mathrm{H}_{3} \mathrm{BrO}$ & & $\mathrm{C}_{8}$ \\
\hline \hline Bond & Average & Angle & Average \\
\hline & & & \\
$\mathrm{Co}$ & $1.181 \mathrm{~B}$ & $\mathrm{OCBr}$ & $122.3 \mathrm{D}$ \\
$\mathrm{CBr}$ & $1.974 \mathrm{~B}$ & $\mathrm{CCBr}$ & $111.0 \mathrm{D}$ \\
$\mathrm{CC}$ & $1.516 \mathrm{D}$ & $\mathrm{HCH}$ & $109.9 \mathrm{D}$ \\
$\mathrm{CH}$ & $1.092^{\mathrm{a}}$ & $\phi^{\mathrm{b}}$ & $1.9 \mathrm{D}$ \\
\hline
\end{tabular}

Methyl group has been assumed to be symmetrical.

- Assumed value from acetyl chloride.

$b$ Methyl tilt angle; toward the oxygen.

[1] S. Tsuchiya and T. Iijima, J. Mol. Struct. 13, 327 (1972).

[2] L. C. Krisher, J. Chem. Phys. 33, 1237 (1970).

\section{Methylbromoform}

\begin{tabular}{cccc}
$\mathrm{C}_{2} \mathrm{H}_{3} \mathrm{Br}_{3}$ & $\mathrm{H}_{3} \mathrm{CCBr}_{3}$ & $\mathrm{C}_{30}$ \\
\hline Bond & Effective & Angle & Effective \\
\hline $\mathrm{CBr}$ & $1.927 \mathrm{D}$ & $\mathrm{CCBr}$ & $107.7 \mathrm{D}$ \\
\hline
\end{tabular}

Methyl group structure and CC distance were assumed from analogous molecules.

[1] Y. S. Li, K. L. Kizer and J. R. Durig, J. Mol. Spectrosc., 42, 430 (1972). Chloroethylene (Vinyl Chloride)

\begin{tabular}{|c|c|c|c|c|c|}
\hline $\mathrm{C}_{2} \mathrm{H}_{3} \mathrm{Cl}$ & & & & & $\mathrm{C}_{\mathrm{e}}$ \\
\hline Bond & $\begin{array}{c}\text { Substitu- } \\
\text { tion }\end{array}$ & Effective & Angle & $\begin{array}{l}\text { Substitu- } \\
\text { tion }\end{array}$ & Effective \\
\hline $\mathrm{CC}$ & $1.333 \mathrm{~B}$ & & $\mathrm{CCCl}$ & $122.7 \mathrm{C}$ & \\
\hline $\mathrm{CCl}$ & $1.726 \mathrm{C}$ & & $\mathrm{CCH}_{2}$ & & $123.0 \mathrm{~B}$ \\
\hline $\mathrm{CH}_{1}$ & & $1.080 \mathrm{C}$ & $\mathrm{CCH}_{2}$ & & $120.6 \mathrm{C}$ \\
\hline $\mathrm{CH}_{2}$ & & $1.070 \mathrm{C}$ & $\mathrm{CCH}_{3}$ & & $121.0 \mathrm{C}$ \\
\hline $\mathrm{CH}_{3}$ & & $1.080 \mathrm{C}$ & & & \\
\hline
\end{tabular}

The $\mathrm{CCCl}$ angle and the distances and angles involving the $\mathrm{H}$ atoms have been recalculated from the original data.

[1] D. Kivelson, E. B. Wilson, Jr, and D. R. Lide, Jr., J. Chem. Phys. 32, 205 (1960). 
3-Methyl-3-chlorodiazirine

(Methylchlorodiazirine)

$\underbrace{\mathrm{C}_{\mathrm{NN}}^{\mathrm{C}_{3} \mathrm{ClN}_{2}}}_{\text {BN }} \stackrel{\text { Effective }}{\stackrel{\mathrm{N}=\mathrm{N}-\mathrm{CClCH}_{3}}{\mathrm{C}_{\mathrm{s}}}}$

[1] J. E. Wollrab and L. E. Scharpen, J. Chem. Phys. 51, 1584 (1969).

\section{Acełyl chloride}

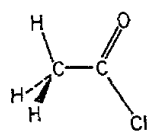

$\mathrm{C}_{2} \mathrm{H}_{3} \mathrm{ClO}$

\begin{tabular}{|c|c|c|c|}
\hline Bond & Substitution & Effective & Average \\
\hline $\begin{array}{l}\mathrm{CC} \\
\mathrm{CCl} \\
\mathrm{CO} \\
\mathrm{CH}\end{array}$ & $1.789 \mathrm{~B}$ & $\begin{array}{l}1.499 \mathrm{D} \\
1.192 \mathrm{D} \\
1.083 \mathrm{D}\end{array}$ & $\begin{array}{l}1.505 \mathrm{~B} \\
1.796 \mathrm{~B} \\
1.185 \mathrm{~B} \\
1.092 \mathrm{~B}\end{array}$ \\
\hline Angle & Substitution & Effective & Average \\
\hline $\begin{array}{l}\mathrm{CCO} \\
\mathrm{CCCl} \\
\mathrm{HCH} \\
\theta^{\mathrm{a}}\end{array}$ & $127.1 \mathrm{~A}$ & $\begin{array}{l}112.7 \mathrm{C} \\
109.8 \mathrm{D}\end{array}$ & $\begin{array}{r}127.2 \mathrm{~B} \\
111.6 \mathrm{C} \\
108.8 \mathrm{C} \\
1.3 \mathrm{C}\end{array}$ \\
\hline
\end{tabular}

Methyl group has been assumed to be symmetrical.

a Methyl group tilt angle; toward the oxygen.

[1] S. Tsuchiya and T. Tijima, J. Mol. Struct. 13, 327 (1972).

[2] K. M. Sinnott, J. Chem. Phys. 34, 851 (1961).

\section{Chloroacetic acid}

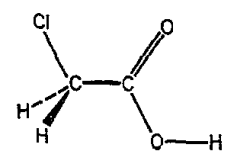

\section{Methylchloroformale}

$\mathrm{C}_{\mathrm{s}}$

\begin{tabular}{|c|c|c|c|c|c|}
\hline Bond & $\begin{array}{l}\text { Substitu- } \\
\text { tion }\end{array}$ & Effective & Angle & $\begin{array}{l}\text { Substitu- } \\
\text { tion }\end{array}$ & Effective \\
\hline $\begin{array}{l}\mathrm{C}_{2} \mathrm{Cl} \\
\mathrm{C}_{2} \mathrm{O}_{1} \\
\mathrm{C}_{1} \mathrm{O}_{1} \\
\mathrm{C}_{1} \mathrm{H}_{8} \\
\mathrm{C}_{1} \mathrm{H}_{8}\end{array}$ & $\begin{array}{l}1.072 \mathrm{C} \\
1.076 \mathrm{C}\end{array}$ & $\begin{array}{l}1.73 \mathrm{D} \\
1.36 \mathrm{D} \\
1.43 \mathrm{D}\end{array}$ & $\begin{array}{l}\mathrm{ClC}_{2} \mathrm{O}_{2} \\
\mathrm{O}_{1} \mathrm{C}_{2} \mathrm{O}_{2} \\
\mathrm{C}_{1} \mathrm{O}_{3} \mathrm{C}_{2} \\
\mathrm{O}_{1} \mathrm{C}_{3} \mathrm{H}_{3} \\
\mathrm{O}_{1} \mathrm{C}_{1} \mathrm{H}_{\mathrm{a}} \\
\mathrm{H}_{3} \mathrm{C}_{4} \mathrm{H}_{3} \\
\mathrm{H}_{2} \mathrm{C}_{3} \mathrm{H}_{2}\end{array}$ & $\begin{array}{l}111.1 \mathrm{C} \\
110.4 \mathrm{C}\end{array}$ & $\begin{array}{l}126 \mathrm{D} \\
125 \mathrm{D} \\
115 \mathrm{D} \\
105 \mathrm{D} \\
110 \mathrm{D}\end{array}$ \\
\hline
\end{tabular}

The effective parameters were calculated under the assumption that the $\mathrm{C}_{2} \mathrm{O}_{2}$ bond length is $1.19 \AA$.

[I] J. R. Durig and M. C. Criffin, J. Mol. Speotrose. 64, 252 (1977).

[2] D. G. Lister and N. L. Owen, J. Chem. Soc. Faraday Trans. II 69, 1036 (1973).

\section{Methylchloroform}

\begin{tabular}{cc|cc}
$\mathrm{C}_{2} \mathrm{H}_{3} \mathrm{Cl}_{3}$ & \multicolumn{1}{c}{$\mathrm{H}_{3} \mathrm{CCCl}_{3}$} & $\mathrm{C}_{3 \mathrm{v}}$ \\
\hline \multirow{2}{*}{ Bond } & Effective & Angle & Effective \\
\hline & & & \\
$\mathrm{CH}$ & $1.090 \mathrm{D}$ & $\mathrm{HCH}$ & $110.0 \mathrm{D}$ \\
$\mathrm{CC}$ & $1.541 \mathrm{D}$ & $\mathrm{CCH}$ & $108.9 \mathrm{D}$ \\
$\mathrm{CCl}$ & $1.771 \mathrm{D}$ & $\mathrm{ClCCl}$ & $109.4 \mathrm{D}$ \\
& & $\mathrm{CCCl}$ & $109.6 \mathrm{D}$
\end{tabular}

[1] R. Holm, M. Mitzlaff and H. Hartmann, Z. Naturforsch. 23A, 3071 (1968).

[2] J. R. During, M. M. Chen and Y. S. Li, J. Mol. Struct. 15, 37 (1973).

[3] S. N. Ghosh, R. Tramburulo and W. Gordy, J. Chem. Phys. 20, 605 (1952).

[4] W. Zeil, Z. Elektrochem. 60, 752 (1956).

\section{$\mathrm{C}_{2} \mathrm{H}_{3} \mathrm{ClO}_{2}$}

Only the conformation has been reliably established.

[1] B. P. Van Eijck, A. A. J. Maagdenberg and J. Wanrooy, J. Mol. Struct., 22, 61 (1974). 
Fluoroethylene (Vinyl Fluoride)

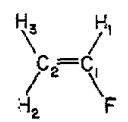

$\mathrm{C}_{2} \mathrm{H}_{3} \mathrm{~F}$

\begin{tabular}{|c|c|c|c|c|c|}
\hline Bond & $\begin{array}{l}\text { Substitu- } \\
\text { tion }\end{array}$ & Effeetive & Angle & $\begin{array}{l}\text { Substitu- } \\
\text { tion }\end{array}$ & Effective \\
\hline $\mathrm{C}_{1} \mathrm{C}_{2}^{\mathrm{a}}$ & & $1.329 \mathrm{D}$ & FCC & & $120.8 \mathrm{D}$ \\
\hline$C_{1} F$ & & $1.347 \mathrm{D}$ & $\mathrm{FCH}_{4}$ & & $110.0 \mathrm{~F}$ \\
\hline $\mathrm{C}_{1} \mathrm{H}_{1}$ & & $1.082 \mathrm{D}$ & $\mathrm{H}_{2} \mathrm{CC}^{\mathrm{a}}$ & & $120.9 \mathrm{D}$ \\
\hline $\mathrm{C}_{2} \mathrm{H}_{2}$ & $1.087 \mathrm{C}$ & & $\mathrm{H}_{3} \mathrm{CC}^{2}$ & & $119.0 \mathrm{D}$ \\
\hline $\mathrm{C}_{2} \mathrm{H}_{3}$ & $1.077 \mathrm{C}$ & & $\mathrm{H}_{3} \mathrm{C}_{2} \mathrm{H}_{2}$ & $120.1 \mathrm{C}$ & \\
\hline
\end{tabular}

- These parameters are near-substitution values. All coordinates except one coordinate of $\mathrm{C}_{1}$ are substitution values.

[1] D. R. Lide, Jr., and D. Christensen, Spectrochim. Acta, 17, 665 (1961).

\section{Acetyl Fluoride}

\begin{tabular}{cc|cc}
$\mathrm{C}_{2} \mathrm{H}_{3} \mathrm{FO}$ & \multicolumn{2}{c}{$\mathrm{CH}_{3} \mathrm{COF}$} & $\mathrm{C}_{\mathrm{B}}$ \\
\hline Bond & Effective & Angle & Effective \\
\hline & & & \\
$\mathrm{CC}$ & $1.502 \mathrm{~B}$ & $\mathrm{CCF}$ & $110.7 \mathrm{D}$ \\
$\mathrm{CF}$ & $1.343 \mathrm{D}$ & $\mathrm{CCO}$ & $127.9 \mathrm{D}$ \\
$\mathrm{CO}$ & $1.185 \mathrm{C}$ & $\mathrm{CCH}$ & $110.4 \mathrm{E}$ \\
$\mathrm{CH}_{\mathrm{i}}$ & $1.082 \mathrm{C}$ & $\mathrm{CCH}_{\mathrm{i}}$ & $108.8 \mathrm{E}$ \\
$\mathrm{CH}_{\mathrm{o}}$ & $1.096 \mathrm{C}$ & $\mathrm{H}_{3} \mathrm{CHi}_{1}$ & $110.8 \mathrm{E}$ \\
& & $\mathrm{H}_{\mathrm{i}} \mathrm{CH}_{\mathrm{o}}$ & $107.3 \mathrm{E}$ \\
\hline
\end{tabular}

$H_{i}$ and $H_{o}$ refer to in plane and out of plane hydrogens. The oxygen atom eclipses a methyl hydrogen.

[1] L. Pieree and L. C. Krisher, J. Chem. Phys. 31, 875 (1959).

\section{cis-Fluoroacetic acid}

$\mathrm{C}_{2} \mathrm{H}_{3} \mathrm{FO}_{2}$

$\mathrm{CH}_{2} \mathrm{FCO}_{2} \mathrm{H}$

C.

cis conformer definitely established from microwave spectra, but insufficient data are available for detailed structure determination.

[1] B. P. van Eijck, G. van der Pìats and P. H. van Roon, J. Mol. Struct. 11, 67 (1972).
trans-Fluoroacetic acid

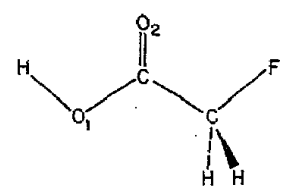

\begin{tabular}{|c|c|}
\hline Bond & Substitution \\
\hline $\mathrm{O}_{1} \mathrm{H}$ & $0.973 \mathrm{C}$ \\
\hline $\mathrm{O}_{1} \mathrm{O}_{2}^{\mathrm{g}}$ & $2.258 \mathrm{~A}$ \\
\hline $\mathrm{O}_{2} \mathrm{H}^{\mathrm{a}}$ & $2.301 \mathrm{C}$ \\
\hline
\end{tabular}

The existence of both cis and trans forms has been definitely establighed from microwave spectra.

These are non-bonding distances.

[1] B. P. van Eijek, C. van der Plaats and P. H. var Foon, J. Mol. Struct. 11, 67 (1972).

\section{1,1,2-Trifluoroethane}

$\mathrm{C}_{2} \mathrm{H}_{3} \mathrm{~F}_{3}$

$\mathrm{HF}_{2} \mathrm{C}-\mathrm{CH}_{2} \mathrm{~F}$

$\mathrm{C}_{1}$

The stable conformation was shown to be the unsymmetrical gauche form.

[1] I. A. Mukhtarov, Soviet Phys. Doklady 8, 72 (1963).

[2] I. A. Mukbtarov, Soviet Phys. Doklady 8, 808 (1964).

\section{Acefonitrile}

$=\mathrm{C}_{2 \mathrm{H}_{3} \mathrm{~N}}^{\mathrm{CH}_{3} \mathrm{CN}}=$

\begin{tabular}{ll|ll}
\hline \hline Bond & Substitution & Angle & Substitution \\
\hline & & & \\
CN & $1.157 \mathrm{~B}$ & HCC & $109.5 \mathrm{~B}$ \\
$\mathrm{CC}$ & $1.458 \mathrm{~B}$ & DCC & $109.5 \mathrm{~B}$ \\
$\mathrm{CH}$ & 1.104 B & & \\
\hline
\end{tabular}

[1] C. C. Costain, J. Chem. Phys., 29, 864 (1968).

[2] C. Matsumura, E. Hirota, T. Oka, and Y. Morino, J. Mol. Spectrose. 9, 366 (1962).

\section{Acetonitrile-N-oxide}

\begin{tabular}{ll}
$\stackrel{\mathrm{C}_{2} \mathrm{H}_{3} \mathrm{NO}}{\mathrm{H}_{3} \mathrm{C}-\mathrm{C}=\mathrm{N}-\mathrm{O}}$ & Substitution \\
\hline Bond & $1.442 \mathrm{~A}$ \\
$\mathrm{CC}$ & $1.169 \mathrm{~B}$ \\
$\mathrm{CN}$ & $1.217 \mathrm{~A}$ \\
$\mathrm{NO}$ &
\end{tabular}

No data are available for $\mathrm{H}$ parameters.

[I] H. K. Bodenseh and K. Morgenstern, Z. Naturforsch. A 25, I50 (1970). 


\section{Nitroethylene}

$\mathrm{C}_{2} \mathrm{H}_{3} \mathrm{NO}_{2}$
$\mathrm{C}_{\mathrm{s}}$

Molecule was shown to be planar.

[1] H. Hess, A. Bander and Hs. H. Günthard, J. Mol. Spectrosc. 22,208 (1967).

\section{1,2,4-Triazole}

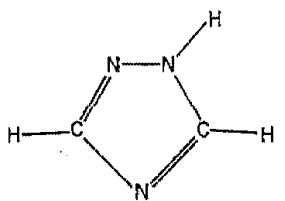

$\mathrm{C}_{2} \mathrm{H}_{2} \mathrm{~N}_{3}$

Unsymmetrical form is principal species in vapor phase. Molecule is essentially planar.

[1] K. Bolton, R. D. Brown, F. R. Burden, and A. Mishra, J. Mol. Struct. 27, 261 (1975)

[2] K. Bolton, R. D. Brown, F. R. Burden, and A. Mishra, Chem. Commun. 1971, 873 (1971).

\begin{tabular}{|c|c|c|c|}
\hline \multicolumn{4}{|c|}{ Ethylene } \\
\hline \multicolumn{2}{|l|}{$\mathrm{C}_{2} \mathrm{H}_{4}$} & \multicolumn{2}{|c|}{$\mathrm{H}_{n} \mathrm{C}=\mathrm{CF}$ ? } \\
\hline Bond & Eflective & Angle & Effective \\
\hline $\mathrm{CH}$ & $1.085 \mathrm{~B}$ & $\mathrm{HCH}$ & $117.8 \mathrm{~B}$ \\
\hline $\mathrm{CC}$ & $1.339 \mathrm{~B}$ & & \\
\hline
\end{tabular}

$\lfloor\perp\rfloor$ J. L. Duncan, I. J. Wright and D. Van Lergerghe, J. Mol. Spectrose. 42,463 (1972).

[2] D. Van Lerberghe, I. I. Wright and J. L. Duncan, J. Mol. Spectrosc. 42, 251 (1972).

\section{1-Chloro-2-fluoroethane}

$\mathrm{C}_{2} \mathrm{H}_{4} \mathrm{CIF}$

$\mathrm{ClCH}_{2}-\mathrm{CH}_{2} \mathrm{~F}$

$\mathrm{C}_{1}$

The observed spectrum indicated a gauche rotamer with a (CCF)(CCCI) dihedral angle of $68^{\circ}(\mathrm{X})$.

[1] I. A. Mukhtarov, E. S. Mukhtarov and L. A. Akhundova, J. Struct. Chem. 7, 565 (1966).

\section{2-Fluoronceiamide}

$\mathrm{C}_{2} \mathrm{H}_{4} \mathrm{FNO}$

$\left.\mathrm{FCH}_{2}{ }^{\prime} \mathrm{CO}\right) \mathrm{NH}_{2}$

$\mathrm{C}_{\mathrm{6}}$

Microwave data showed that the heavy atom skeleton and the amino hydrogens lie in a plane, and that the $C=0$ and $C-F$ bonds were in the trans conformation.

[1] K. M. Marstokk and H. Mollendal, J. Mol. Struct. 22, 287 (1974)

\section{gauche 1,2-Difluoroethane}

$\mathrm{CH}_{2} \mathrm{FCH}_{2} \mathrm{~F}$

$\mathrm{C}_{1}$

Dihedral angle between the two CCF planes is $73^{\circ}(\mathrm{X})$.

[1] S. Butcher, R. Cohen, and T. C. Rounds, J. Chem. Phys. 54, $41.23(197 \mathrm{~J})$.

trans-Trifluoroethylamine

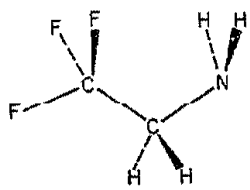

$\mathrm{C}_{2} \mathrm{H}_{4} \mathrm{~F}_{3} \mathrm{~N}$

Molecule was shown to exist in the trans conformation, but detailed structure determination was not possible.

[1] I, D. Warren and E. B. Wilson, J. Chem. Phys., 56, 2137 (1972).

Ethynyigermane (Germylacetylene)

\begin{tabular}{|c|c|c|c|c|c|}
\hline $\mathrm{C}_{2} \mathrm{H}_{4} \mathrm{Ge}$ & \multicolumn{4}{|c|}{$\mathrm{H}_{3} \mathrm{GeC} \equiv \mathrm{CH}$} & $\mathrm{C}_{\hat{\imath} \mathrm{v}}$ \\
\hline Bond & $\begin{array}{l}\text { Substitu- } \\
\text { tion }\end{array}$ & Effective & Angle & $\begin{array}{c}\text { Substitu- } \\
\text { tion }\end{array}$ & Effective \\
\hline $\mathrm{GeC}$ & $1.896 \mathrm{~B}$ & & $\mathrm{HGeH}$ & & $109.2 \mathrm{X}$ \\
\hline $\mathrm{CC}$ & $1.208 \mathrm{~A}$ & & & & \\
\hline GeH & & $1.521 \times$ & & & \\
\hline
\end{tabular}

The CH distance has been assumed to be $1.056 \AA$.

[1] E. C. Thomas and V. W. Laurie, J. Chem. Phys. 44, 2602 (1966)

\section{A mino acetoniłrile}

$\mathrm{C}_{2} \mathrm{H}_{4} \mathrm{~N}_{2}$

$\mathrm{H}_{2} \mathrm{~N}-\mathrm{CH}_{2} \mathrm{CN}$

$\mathrm{C}_{8}$

The amino group is found in the conformation in which the amino protons are trans to the methylene protons.

[1] H. Pickett, J. Mol. Spectrosc. 46, 335 (1973).

[2] J. N. McDonald and J. K. Tyler, J.C.S. Chem. Comm. 1972, $995(1972)$ 
3-Methyldiazirine

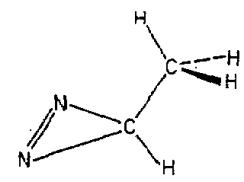

$\mathrm{C}_{2} \mathrm{H}_{4} \mathrm{~N}_{2}$

\begin{tabular}{llll}
\hline Distance & Substitution & Effective \\
\hline $\mathrm{NM}$ & & $1.235 \mathrm{C}$ \\
$\mathrm{H}_{2} \mathrm{H}_{2}^{\mathrm{a}}$ & $1.771 \mathrm{~B}$ & &
\end{tabular}

The equilibrium orientation of the methyl group is staggered.

S Refers to non-bonding $\mathrm{H} \cdots \mathrm{H}$ distance in methyl group.

[1] L. H. Scharpen, J. E. Wollrab, D. P. Ames, and J. A. Merritt, J. Chem. Phys. 50, 2063 (1969).

[2] D. W. Gord and J. E. Wollrab, J. Chem. Phys. 51, 5728 (1969).

\section{Aceialdehyde}

\begin{tabular}{lllllll} 
& & & \\
& & & & & \\
\hline
\end{tabular}

The effective parameters were recalculated from reported data. The $\mathrm{CCH}_{1}$ angle was assumed to be $117.5^{\circ}$ and a symmetrical methyl group was assumed in the determination of the average parameters.

[1] T. Iijime and M. Kimura, Bull. Chem. Soc. Japan, 42, 2159 (1969).

[2] R. W. Kilb, C. C. Lin, and E. B. Wilson, Ir., J. Chem. Phys. 26, 1695 (1957).
1,2-Epoxyethane

(Elhylene oxide or oxirane)

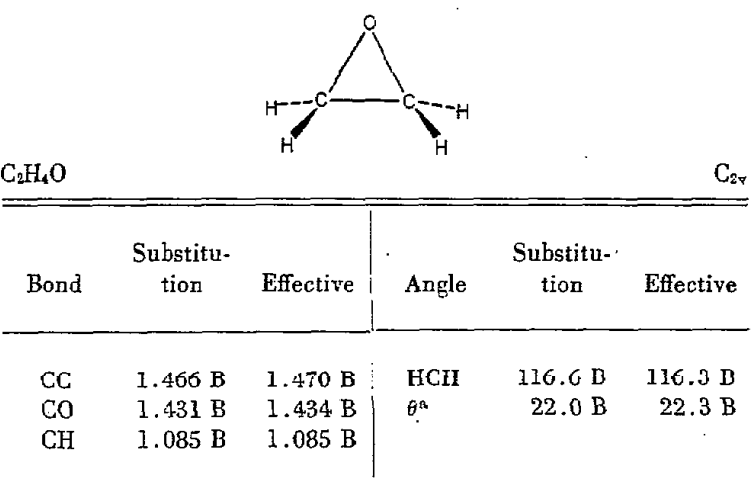

s Angle between CC bond and the line which bisects the HCH angle.

[1] C. Hirose, Bull. Chem. Soc. Japan 47, 1311 (1974)

[2] G. L. Cunningham, A. W. Boyd, R. J. Myers and W. D. Gwinn, J. Chem. Phys. 19; 670 (1951).

\section{Methyl thiolformate}

$\mathrm{C}_{2} \mathrm{H}_{4} \mathrm{OS}$

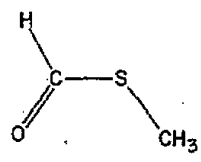

$\mathrm{C}_{\mathrm{s}}$

Microwave data are consistent with a cis conformation.

[1] G. I. L. Jones, D. G. Lister, N. L. Uwen, M. C. L. Gerry and P. Palmieri, J. Mol. Spectrosc. 60,348 (1976).

Thiirane-1-oxide (Ethylene episulfoxide)

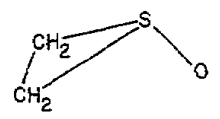

$\mathrm{C}_{2} \mathrm{H}_{4} \mathrm{OS}$

$\mathrm{C}_{8}$

\begin{tabular}{ll|lr}
\hline Bond & Effective & Angle & Effective \\
\hline SO & $1.483 \mathrm{D}$ & OSC & $110.0 \mathrm{D}$ \\
CS & $1.822 \mathrm{D}$ & CSC & $48.8 \mathrm{D}$ \\
CC & $1.504 \mathrm{D}$ & & \\
\hline
\end{tabular}

Assumptions: $\mathrm{CH}=1.078, \mathrm{HCH}=116.0, \mathrm{HCC}=151.7$.

[1] S. Saito, Bull. Chem. Soc. Japan 42, 663 (1969). 


\section{Acetic acid}

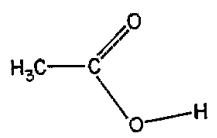

$\mathrm{C}_{2} \mathrm{H}_{4} \mathrm{O}_{2}$

Microwave data are consistent only with the above conformation

[1] T. C. Krisher and F. Saegeharth, I. Chem. Phys. 54, 4553 (1971).

[2] W. 3. Tabor, J. Chem. Phys. 27, 974 (1957).

\section{Glycolaldehyde}

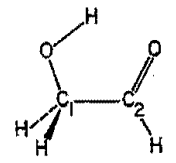

$\mathrm{C}_{2} \mathrm{H}_{4} \mathrm{O}_{2}$

\begin{tabular}{ll|ll}
\hline \hline Bond & Substitution & Angle & Substitution \\
\hline & & & \\
\cline { 3 - 3 } $\mathrm{C}_{2} \mathrm{O}$ & $1.209 \mathrm{~A}$ & $\mathrm{C}_{2} \mathrm{C}_{2} \mathrm{O}$ & $122.7 \mathrm{~B}$ \\
$\mathrm{C}_{1} \mathrm{O}$ & $1.437 \mathrm{~B}$ & $\mathrm{C}_{2} \mathrm{C}_{1} \mathrm{O}$ & $111.5 \mathrm{~B}$ \\
$\mathrm{CC}$ & $1.499 \mathrm{~A}$ & $\mathrm{C}_{1} \mathrm{C}_{2} \mathrm{H}$ & $115.3 \mathrm{~B}$ \\
$\mathrm{OH}$ & $1.051 \mathrm{~B}$ & $\mathrm{C}_{2} \mathrm{C}_{2} \mathrm{H}$ & $109.2 \mathrm{~B}$ \\
$\mathrm{C}_{2} \mathrm{H}$ & $1.102 \mathrm{~B}$ & $\mathrm{C}_{1} \mathrm{OII}$ & $101.6 \mathrm{~B}$ \\
$\mathrm{C}_{1} \mathrm{H}$ & $1.093 \mathrm{~B}$ & $\mathrm{HC} \mathrm{C}_{1} \mathrm{H}$ & $107.6 \mathrm{~B}$
\end{tabular}

[1] K. M. Marstokk and H. Mollendal, J. Mol. Struct. 7, 101 (1971).

[2] K. M. Marstokk and H. Mollendal, J. Mol. Struct. 16, 259 (1973).

Methyl formate

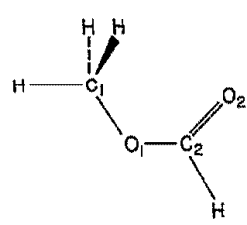

$\mathrm{C}_{2} \mathrm{H}_{4} \mathrm{O}_{2}$

\begin{tabular}{lc|ll}
\hline \hline Bond & Substitution & Angle & Substitution \\
\hline & & & \\
$\mathrm{C}_{2} \mathrm{O}_{2}$ & $1.200 \mathrm{~B}$ & $\mathrm{O}_{1} \mathrm{C}_{2} \mathrm{O}_{2}$ & $125.9 \mathrm{~B}$ \\
$\mathrm{C}_{2} \mathrm{O}_{2}$ & $1.334 \mathrm{~B}$ & $\mathrm{C}_{1} \mathrm{O}_{2} \mathrm{C}_{2}$ & $114.8 \mathrm{~B}$ \\
$\mathrm{C}_{1} \mathrm{O}_{1}$ & $1.437 \mathrm{~B}$ & $\mathrm{HC}_{2} \mathrm{O}_{1}$ & $109.3 \mathrm{~B}$ \\
$\mathrm{C}_{2} \mathrm{H}$ & $1.101 \mathrm{~B}$ & $\mathrm{HC}_{2} \mathrm{H}$ & $110.7 \mathrm{C}$ \\
$\mathrm{C}_{2} \mathrm{H}$ & $1.086 \mathrm{C}$ & & \\
\hline
\end{tabular}

Methyl group has been chosen symmetrical for the reported parameters.

[1] R. F. Curl, Jr., J. Chem. Phys. 30, 1529 (1959).
Thiirane-1,1-dioxide

(Ethylene episulfone)

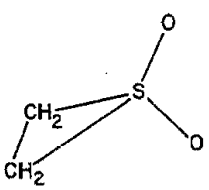

$\mathrm{C}_{2} \mathrm{H}_{4} \mathrm{O}_{2} \mathrm{~S}$

\begin{tabular}{cc|cc}
\hline \hline Bond & Efrective & Angle & Effective \\
\hline SO & $1.439 \mathrm{D}$ & OSO & $121.4 \mathrm{D}$ \\
CS & $2.731 \mathrm{D}$ & CSC & $54.7 \mathrm{D}$ \\
CC & $1.590 \mathrm{C}$ & & \\
\hline
\end{tabular}

Assumptions: $\mathrm{CH}=1.078, \mathrm{HCH}=116.0, \mathrm{HCC}=151.7$.

[1] Y. Nakano, S. Saito and Y. Morino, Bull. Chem. Soc. Japan $43,368(1970)$.

$\mathrm{C}_{\mathrm{s}}$

-

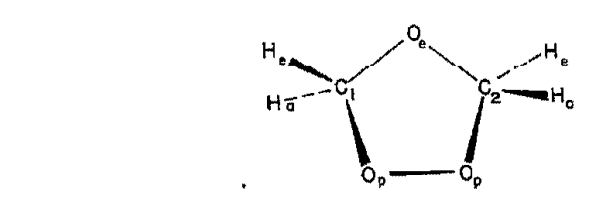

$\mathrm{C}_{2} \mathrm{H}_{4} \mathrm{O}_{3}$

$\mathrm{C}_{2}$

\begin{tabular}{|c|c|c|c|c|c|}
\hline Bond & $\begin{array}{l}\text { Substitu- } \\
\text { tion }\end{array}$ & Angle & $\begin{array}{l}\text { Substitu- } \\
\text { tion }\end{array}$ & $\begin{array}{l}\text { Dihed. } \\
\text { Angle }\end{array}$ & $\begin{array}{c}\text { Substitu- } \\
\text { tion }\end{array}$ \\
\hline $\begin{array}{l}\mathrm{CH}_{\mathrm{a}} \\
\mathrm{CH}_{\mathrm{a}} \\
\mathrm{CO} \\
\mathrm{CO}_{\mathrm{p}} \\
\mathrm{O}_{\mathrm{p}} \mathrm{O}_{\mathrm{p}}\end{array}$ & $\begin{array}{l}1.091 \mathrm{C} \\
1.097 \mathrm{C} \\
1.416 \mathrm{~B} \\
1.412 \mathrm{~B} \\
1.461 \mathrm{~B}\end{array}$ & $\begin{array}{l}\mathrm{COC} \\
\mathrm{COO} \\
\mathrm{OCO} \\
\mathrm{HCH} \\
\mathrm{O}_{0} \mathrm{CH}_{8} \\
\mathrm{O}_{8} \mathrm{CH}_{a} \\
\mathrm{O}_{\mathrm{D}} \mathrm{CH}_{8} \\
\mathrm{O}_{\mathrm{D}} \mathrm{CH}_{\mathrm{B}}\end{array}$ & $\begin{array}{r}104.8 \mathrm{~B} \\
99.3 \mathrm{~B} \\
105.5 \mathrm{~B} \\
113.3 \mathrm{C} \\
110.8 \mathrm{C} \\
109.8 \mathrm{C} \\
106.7 \mathrm{C} \\
110.4 \mathrm{C}\end{array}$ & $\begin{array}{l}\mathrm{C}_{1} \mathrm{O}_{2} \mathrm{C}_{2} \mathrm{O}_{\mathrm{p}} \\
\mathrm{C}_{1} \mathrm{O}_{\mathrm{D}} \mathrm{O}_{\mathrm{D}} \mathrm{C}_{2} \\
\mathrm{O}_{2} \mathrm{CO}_{\mathrm{D}} \mathrm{O}_{\mathrm{p}} \\
\mathrm{C}_{1} \mathrm{O}_{0} \mathrm{C}_{2} \mathrm{H}_{\mathrm{o}} \\
\mathrm{C}_{1} \mathrm{O}_{8} \mathrm{C}_{2} \mathrm{H}_{\mathfrak{a}}\end{array}$ & $\begin{array}{r}-16.2 \mathrm{~B} \\
-49.5 \mathrm{~B} \\
40.8 \mathrm{~B} \\
-131.3 \mathrm{C} \\
102.8 \mathrm{C}\end{array}$ \\
\hline
\end{tabular}

[1] R. L. Kuczkowski, C. W. Gillies, and K. L. Gallaher, J. Mol. Spectrosc. 60, 361 (1976).

[2] U. Mazur and R. L. Kuczkowski, J. Mol. Spectrosc. 65, 84 (1977).

[3] C. W. Gillies and R. L. Kuczkowski, J. Amer. Chem. Soc. 94, 6337 (1972). 
Thiirane

(Ethylene sulfide)

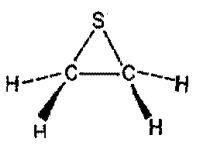

\begin{tabular}{cc|cc}
$\mathrm{C}_{2} \mathrm{H}_{4} \mathrm{~S}$ & & $\mathrm{C}_{28}$ \\
\hline \hline Bond & Substitution & Angle & Substitution \\
\hline & & & \\
$\mathrm{CC}$ & $1.484 \mathrm{~B}$ & $\mathrm{CSC}$ & $48.3 \mathrm{~B}$ \\
$\mathrm{CS}$ & $1.815 \mathrm{~B}$ & $\mathrm{HCH}$ & $115.8 \mathrm{~B}$ \\
$\mathrm{CH}$ & $1.083 \mathrm{~B}$ & $\phi^{\mathrm{n}}$ & $151.8 \mathrm{~B}$ \\
& & & \\
\hline
\end{tabular}

a Angle between HCH bisector and $\mathrm{CC}$ bond.

[I] K. Okiye, C. Hirose, D. G. Lister and J. Sheridan, Chell. Phys. Lett. 24, 111 (1974).

[2] G. L. Cunningham, A. W. Boyd, R. J. Myers, W. D. Gwinn and W. I. LeVan, J. Chem. Phys, 19, 676 (1951).

\section{Thioacetaldehyde}

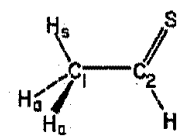

$\mathrm{C}_{2} \mathrm{H}_{4} \mathrm{~S}$

\begin{tabular}{ll|ll}
\hline \hline Bond & Substitution & Angle & Substitution \\
\hline $\mathrm{CS}$ & $1.610 \mathrm{C}$ & $\mathrm{CCS}$ & $125.3 \mathrm{C}$ \\
$\mathrm{CC}$ & $1.506 \mathrm{~B}$ & $\mathrm{C}_{1} \mathrm{C}_{2} \mathrm{H}$ & $119.4 \mathrm{C}$ \\
$\mathrm{C}_{2} \mathrm{H}$ & $1.089 \mathrm{~B}$ & $\mathrm{C}_{2} \mathrm{C}_{1} \mathrm{H}_{3}$ & $111.2 \mathrm{C}$ \\
$\mathrm{C}_{1} \mathrm{H}_{3}$ & $1.090 \mathrm{C}$ & $\mathrm{C}_{2} \mathrm{C}_{1} \mathrm{H}^{3}$ & $110.1 \mathrm{C}$ \\
$\mathrm{C}_{1} \mathrm{H}_{2}$ & $1.098 \mathrm{D}$ & & \\
\hline
\end{tabular}

[1] H. W. Kroto and B. M. Landsberg, J. Mol. Spectrosc. 62, 346 (1976).

Ethynyisilane (Silyl Acetylene)

\begin{tabular}{cc|cc}
$\mathrm{C}_{2} \mathrm{H}_{4} \mathrm{Si}$ & & $\mathrm{H}_{3} \mathrm{SiC}=\mathrm{CH}$ & $\mathrm{C}_{3 \mathrm{v}}$ \\
\hline Bond & Substitution & . Angle & Substitution \\
\hline $\mathrm{SiC}$ & $1.826 \mathrm{~B}$ & $\mathrm{HSiH}$ & $110.2 \mathrm{~A}$ \\
$\mathrm{CC}$ & $1.208 \mathrm{~A}$ & & \\
$\mathrm{CH}$ & $1.058 \mathrm{~A}$ & & \\
$\mathrm{SiH}$ & $1.488 \mathrm{~A}$ & &
\end{tabular}

[1] M. C. L. Gerry and T. M. Sugden, Trans. Faraday Soc. 61, 2091 (1965).

[2] J. S. Muenter and V. W. Laurie, J. Chem. Phys. 39, 1181 (1963).
1,3,2-Dioxaborolane

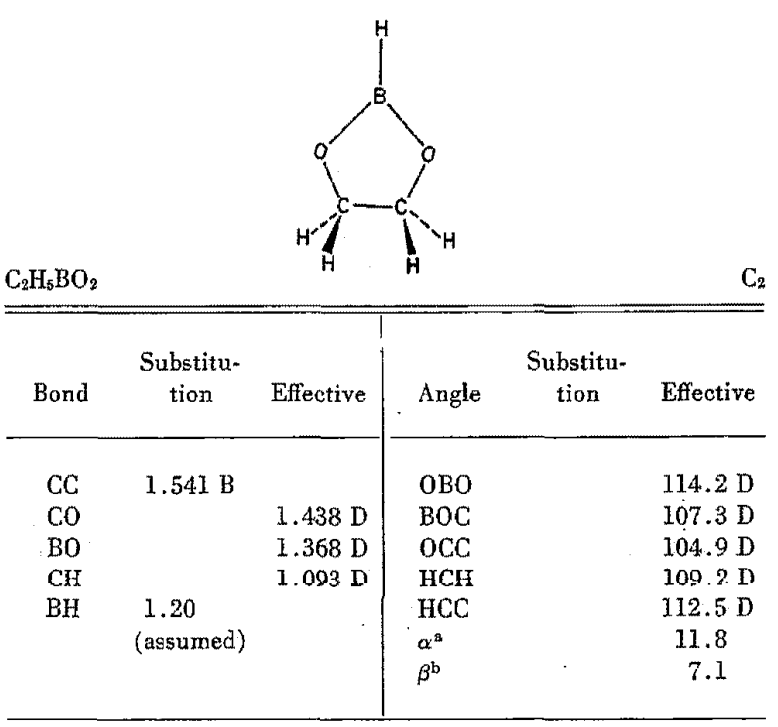

${ }^{B} \alpha$ is angle between the two CCO planes.

$b \beta$ is angle between CBC and OBO planes. HCH assumed to be bisected by OCC plane.

[1] J. H. Hund and R. H. Schwendeman, J. Chem. Phys. 45, 3349 (1966).

2-Chlaro-1,6-dicarbahexaborane (6)

\begin{tabular}{|c|c|c|c|}
\hline $\mathrm{C}_{2} \mathrm{H}_{5} \mathrm{~B}_{4} \mathrm{Cl}$ & & & $\mathrm{C}_{2}$ \\
\hline Bond & Substitution & Angle & Substitution \\
\hline $\mathrm{B}_{1} \mathrm{Cl}$ & $1.823 \mathrm{D}$ & $B_{1} B_{2} B_{z}$ & $87.7 \mathrm{C}$ \\
\hline $\mathrm{B}_{1} \mathrm{~B}_{2}$ & $1.671 \mathrm{D}$ & $\mathrm{B}_{2} \mathrm{~B}_{3} \mathrm{~B}_{4}$ & $91.0 \mathrm{C}$ \\
\hline $\mathrm{B}_{2} \mathrm{~B}_{3}$ & $1.702 \mathrm{C}$ & $\mathrm{B}_{2} \mathrm{~B}_{1} \mathrm{~B}_{4}$ & $93.6 \mathrm{C}$ \\
\hline
\end{tabular}

In above structural drawing, each boron (except $B_{i}$ ) and the two carbons have one bonded hydrogen atom.

[1] G. L. Mckown and R. A. Beaudet, Inorg. Chem. 10, 1350 (1971). 
Bromoethane

(Ethyl bromide)

\begin{tabular}{cc|cc}
$\mathrm{C}_{2} \mathrm{H}_{6} \mathrm{Br}$ & \multicolumn{1}{c}{$\mathrm{H}_{3} \mathrm{C}^{2} \mathrm{C}^{2} \mathrm{H}_{2} \mathrm{Br}$} & $\mathrm{C}_{8}$ \\
\hline Bond & Substitution & Angle & Substitution \\
\hline & & & \\
$\mathrm{CBr}$ & $1.950 \mathrm{C}$ & $\mathrm{CCBr}^{2}$ & $111.0 \mathrm{C}$ \\
$\mathrm{CC}$ & $1.518 \mathrm{~B}$ & $\mathrm{C}^{1} \mathrm{C}^{2} \mathrm{H}$ & $112.2 \mathrm{C}$ \\
$\mathrm{C}^{\top} \mathrm{H}$ & $1.093 \mathrm{C}$ & $\mathrm{HC}^{2} \mathrm{H}$ & $109.9 \mathrm{C}$ \\
$\mathrm{C}^{2} \mathrm{H}$ & $1.087 \mathrm{C}$ & $\mathrm{HC}^{1} \mathrm{H}$ & $108.9 \mathrm{C}$ \\
\hline
\end{tabular}

Methyl group was assumed to be symmetrical.

[1] C. Flanagan and L. Pierce, J. Chem. Phys. 38, 2963 (1963).

\section{Chloroethane \\ (Ethyl chloride)}

\begin{tabular}{ll|ll}
$\mathrm{C}_{2} \mathrm{H}{ }_{5} \mathrm{Cl}$ & & $\mathrm{CH}_{3} \mathrm{CH}_{2} \mathrm{Cl}$ & \\
\hline & & & \\
Bond & Substitution & Angle & Substitution \\
& & & \\
$\mathrm{CC}$ & $1.520 \mathrm{~B}$ & $\mathrm{CCCl}$ & $111.0 \mathrm{~A}$ \\
$\mathrm{CCl}$ & $1.788 \mathrm{~A}$ & $\mathrm{HCH}$ (methyl) & $108.5 \mathrm{C}$ \\
$\mathrm{CH}$ (methyl) & $1.091 \mathrm{C}$ & $\mathrm{HCH}$ (methylene) & $109.2 \mathrm{C}$ \\
$\mathrm{CH}$ (methylene) & $1.089 \mathrm{C}$ & $\mathrm{CCH}$ (methylene) & $111.6 \mathrm{C}$
\end{tabular}

Conformation is staggered. $\mathrm{CH}_{3}$ group is assumed to be symmetrical.

[1] R. H. Schwendeman and G. D. Jacobs, J. Chem. Phys. 36, 1245 (1962).

[2] R. S. Wagner and B. P. Dailey, J. Chem. Phys, 26, 1588 (1957).

\section{Chloroethanol}

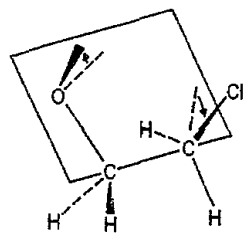

$\mathrm{C}_{2} \mathrm{H}_{5} \mathrm{ClO}$

\begin{tabular}{ll|ll}
\hline Bond & Substitution & Angle & $\begin{array}{c}\text { Substitu- } \\
\text { tion }\end{array}$ \\
\hline $\mathrm{CCI}$ & $1.789^{\mathrm{s}}$ & $\mathrm{CCCl}$ & $110.1 \mathrm{X}$ \\
$\mathrm{CC}$ & $1.519 \mathrm{X}$ & $\mathrm{CCO}$ & $112.8 \mathrm{X}$ \\
$\mathrm{CO}$ & $1.411 \mathrm{~B}$ & $\mathrm{COH}$ & $105.8 \mathrm{C}$ \\
$\mathrm{OII}{ }_{1}$ & $1.008 \mathrm{C}$ & $(\mathrm{CCO})-\left(\mathrm{CCCl}^{\mathrm{b}}\right)^{\mathrm{b}}$ & $63.2 \mathrm{X}$ \\
$\mathrm{Cl} \cdots \mathrm{H}_{1}$ & $2.609 \mathrm{~B}$ & $(\mathrm{CCO})-\left(\mathrm{COH}_{\mathrm{I}}\right)^{\mathrm{b}}$ & $58.4 \mathrm{X}$ \\
\hline
\end{tabular}

a Assumed value.

b Dihedral angles between indicated planes.

[1] R. G. Azrak and E. B. Wilson, J. Chem. Phys. 52, 5299 (1970).
Fluoroethane

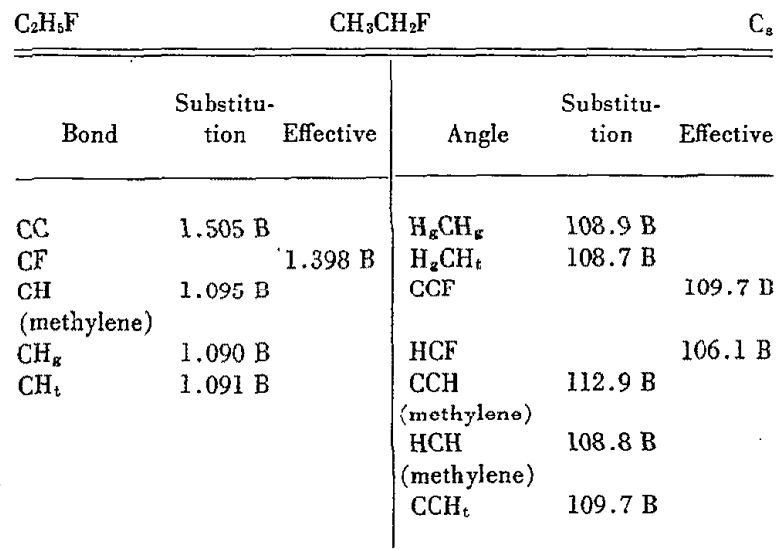

Conformation is staggered. $t$ and $g$ refer to trans and gauche positions of methyl group protons with respect to $F$ atom.

[1] L. Nygaard, Spectrochim. Acta 22, 1261 (1966).

[2] B. Bak, S. Detoni, L. Hanser-Nygaard, J. T. Nielsen and J. Rastrup-Andersen, Spectrochim. Acta 16, 376 (1960).

\section{Nitroethane}

$\mathrm{C}_{2} \mathrm{H}_{3} \mathrm{NO}_{2}$

$\mathrm{CH}_{3} \mathrm{CH}_{2} \mathrm{NO}_{2}$

$\mathrm{C}_{6}$

Planarity of heavy atoms is a well-confirmed feature.

[1] Krishnaji and G. K. Panday, Indian J. Pure and App. Phys. 8, $261(1970)$

Phosphirane

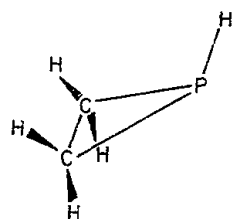

$\mathrm{C}_{2} \mathrm{H}_{5} \mathrm{P}$

$\mathrm{C}_{\mathrm{s}}$

\begin{tabular}{ll|ll}
\hline Bond & Substitution & Angle & Substitution \\
\hline & & & \\
PC & $1.867 \mathrm{~B}$ & $\mathrm{HPC}$ & $95.2 \mathrm{~B}$ \\
$\mathrm{rH}$ & $1.428 \mathrm{~B}$ & $\mathrm{CCH}_{\text {cis }}$ & $118.0 \mathrm{~B}$ \\
$\mathrm{CC}$ & $1.502 \mathrm{~B}$ & $\mathrm{CCH}_{\text {irans }}$ & $117.5 \mathrm{~B}$ \\
$\mathrm{CH}_{\text {cis }}$ & $1.092 \mathrm{~B}$ & $\mathrm{HCH}$ & 114.4 \\
$\mathrm{CH}_{\text {trans }}$ & $1.093 \mathrm{~B}$ & & \\
\end{tabular}

[1] M. T. Bowers, R. A. Beaudet, H. Goldwhite and R. Tang, J. Amer. Chem. Soc. 91, 17 (1969). 
Merhyl Isocyonicizarone

\begin{tabular}{|c|c|c|c|}
\hline $\mathrm{C}_{2} \mathrm{H}_{6} \mathrm{BN}$ & $\mathrm{CH}$ & $\mathrm{BW}_{3}$ & \\
\hline Bond & Substitution & Angle & Effective \\
\hline$C 1 N$ & $1.416 \mathrm{~B}$ & HCF́ & $110.0 \mathrm{C}$ \\
\hline $\mathrm{NC}^{2}$ & $1.155 \mathrm{C}$ & HBE & $113.0 \mathrm{C}$ \\
\hline \multirow[t]{2}{*}{$\mathrm{C}^{2} \overrightarrow{\mathrm{B}}$} & $1.566 \mathrm{~B}$ & $\mathrm{HBC}$ & $105.7 \mathrm{C}$ \\
\hline & & $\mathrm{HCN}$ & $109.0 \mathrm{C}$ \\
\hline
\end{tabular}

Effective parameiers were obtained by assuming $\mathrm{BH}=1.220 \pm$ 0.020 and $\mathrm{CH}=1.100 \pm 0.015$.

[I] J. F. Stevene, Ir., J. W. Bowan, R. E. Curl, Jr., R. A. Coanangol end M. Grace Hu, I. Amer. Chem. Soc. 99, 1442 (1977).

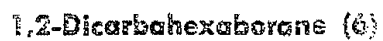

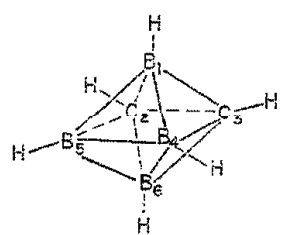

$\mathrm{Cin}_{2} \mathrm{BH}_{4}$

\begin{tabular}{|c|c|c|c|}
\hline Bond & Substiutution & Bond & Substitution \\
\hline $\mathrm{B}_{1} \mathrm{~B}_{\mathrm{s}}$ & $2.43 A B$ & $\mathrm{~B}_{4} \mathrm{C}_{3}$ & $1.605 \mathrm{~B}$ \\
\hline $\mathrm{C}_{2} \mathrm{~B}_{4}$ & $2.297 \mathrm{~B}$ & $\mathrm{C}_{2} \mathrm{C}_{3}$ & $2.540 \mathrm{P}$ \\
\hline $\mathrm{B}_{1} \mathrm{~B}_{4}$ & $1.721 . \mathrm{D}$ & $\mathrm{B}_{1} \mathrm{C}_{2}$ & $1.627 \mathrm{D}$ \\
\hline $\mathrm{B}_{1} \mathrm{~B}_{t}$ & $1.752 \mathrm{~B}$ & & \\
\hline
\end{tabular}

[- R. A. Boeudet and R. L. Poynter, J. Chem. Phys. 33,1899 (1970).

\section{Dimerhylcedmium}

\begin{tabular}{|c|c|c|c|}
\hline \multirow{2}{*}{$\frac{\mathrm{C}_{2} \mathrm{Fi}_{\mathrm{S}} \mathrm{Cu}}{\text { Bond }}$} & \multicolumn{2}{|c|}{$\mathrm{CH}_{3} \mathrm{CdCH}_{3}$} & Undeiermined \\
\hline & Effective & Angle & Effective \\
\hline $\mathrm{Cd}-\mathrm{C}$ & $2.112 \mathrm{C}$ & $\mathrm{HCH}$ & $108.4 \mathrm{Y}$ \\
\hline
\end{tabular}

In Grder to obtain the HCH angle it was necessary to assume the C-H disiance as 1.09 \&.. The heayy atoms were assumed to be colinear.

[1] Ka. S. Rao, E.P. Stoichefí and R. Turner, Can. J. Phys. 39, I516 (1960).
Vinỵgermene

\begin{tabular}{cc|cc}
$\mathrm{C}_{2} \mathrm{H}_{6} \mathrm{Ge}$ & & & $\mathrm{C}_{8}$ \\
\hline \hline Bond & Effecive & Angie & Efective \\
\hline $\mathrm{CC}$ & $1.347 \mathrm{X}$ & $\mathrm{CCGe}$ & $122.9 \mathrm{X}$ \\
$\mathrm{GeC}$ & $1.926 \mathrm{X}$ & $\mathrm{CGeH}$ & $109.7 \mathrm{X}$ \\
$\mathrm{Gell}$ & $1.520 \mathrm{X}$ & &
\end{tabular}

Ail vinyl hydrogen parameters have been assumed and the germyl group has been assumed to be symmetric.

[1] J. R. During, K. L. Kizer and Y. S. Li, J. Amer. Chem. Soc. 96, $7400(1974)$.

\section{Dimefhymercury}

\begin{tabular}{cc|cc}
$\mathrm{C}_{2} \mathrm{H}_{6 \mathrm{Hg}}$ & \multicolumn{2}{c}{$\mathrm{CH}_{3} \mathrm{HgCH}_{2}$} & Undetermined \\
\hline Bond & Effective & Anfle & Efiective \\
\hline $\mathrm{HgC}$ & $2.094 \mathrm{C}$ & $\mathrm{HCH}$ & $109.3 \mathrm{X}$
\end{tabular}

In order to obtain the HCH angle, the $\mathrm{C}-\mathrm{H}$ distance was assumed. The CHgC configuration was assumed to be linear.

[1] K. S. Rao, B. P. Stoicheff and R. Turner, Can, J. Phys. 38, $15 \$ 6$ (1960).

\section{Rembresodimethylemine}

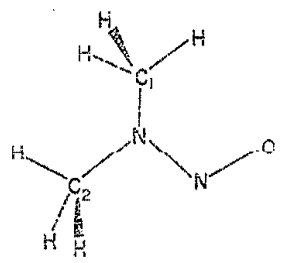

\begin{tabular}{|c|c|c|c|}
\hline Bond & Effective & Angle & Effective \\
\hline NN & $1.329 \mathrm{~J}$ & $\mathrm{NNC}_{\mathrm{I}}$ & $121 . \mathrm{A} C$ \\
\hline No & $1.233 \mathrm{D}$ & $\mathrm{NNCE}$ & $216.1 \mathrm{D}$ \\
\hline $\mathrm{NC}_{1}$ & $1.4 .4 \mathrm{D}$ & NNO & $134.0 \mathrm{C}$ \\
\hline $\mathrm{NC}_{4}$ & $1.452 \mathrm{D}$ & NACH: & III. $2 \mathrm{X}$ \\
\hline $\mathrm{CH}^{2}$ & $1.065 \times$ & & \\
\hline
\end{tabular}

Although several heavy-atom isotopic species rore intestigeted, very snall coordinates yicked a structure of uncertain quality.

a $\mathrm{AICH}$ bonds anc $\mathrm{NCF}$ angles were assumed to he identical.

[1] A. Guemieri, $Z$. Rohner and E. Scappini, $Z$. Naturorsch. 30a. $904(2975)$

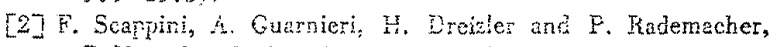
2. Naturorsch. 27a, 1329 (1072). 


\section{gauche-Ethanol}

$\mathrm{C}_{2} \mathrm{H}_{6} \mathrm{O}$

This conformer has $\mathrm{OH}$ group rotated $126^{\circ}$ (X) from the wellestablished trans position.

[1] Y. Sasada, J. Mol. Spectrosc. 38, 33 (1971).

\section{frans-Ethanol}

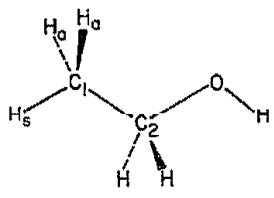

$\mathrm{C}_{2} \mathrm{H}_{6} \mathrm{O}$

\begin{tabular}{ll|ll}
\hline \hline Bond & Substitution & Angle & Substitution \\
\hline $\mathrm{CC}$ & $1.512 \mathrm{~B}$ & $\mathrm{CCO}$ & $107.8 \mathrm{~B}$ \\
$\mathrm{CO}$ & $1.431 \mathrm{~B}$ & $\mathrm{COH}$ & $105.4 \mathrm{~B}$ \\
$\mathrm{OH}$ & $0.971 \mathrm{C}$ & $\mathrm{C}_{1} \mathrm{C}_{2} \mathrm{H}$ & $110.7 \mathrm{~B}$ \\
$\mathrm{C}_{2} \mathrm{H}$ & $1.098 \mathrm{~B}$ & $11 \mathrm{C}_{2} \mathrm{H}$ & $108.0 \mathrm{D}$ \\
$\mathrm{C}_{1} \mathrm{H}_{3}$ & $1.091 \mathrm{C}$ & $\mathrm{C}_{2} \mathrm{C}_{1} \mathrm{H}_{\mathrm{a}}$ & $110.1 \mathrm{~B}$ \\
$\mathrm{C}_{1} \mathrm{H}_{8}$ & $1.088 \mathrm{C}$ & $\mathrm{C}_{2} \mathrm{C}_{1} \mathrm{H}_{\mathrm{s}}$ & $110.5 \mathrm{C}$ \\
& & $\mathrm{H}_{3} \mathrm{C}_{1} \mathrm{H}_{2}$ & $108.4 \mathrm{~B}$ \\
\hline
\end{tabular}

[1] J. P. Culot, Fourth Austin Symposium on Gas Phase Molecular Structure, Paper T8 (1972).

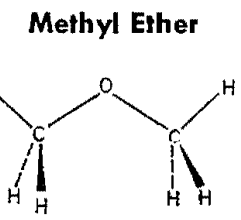

$\mathrm{C}_{2} \mathrm{H}_{6} \mathrm{O}$

\begin{tabular}{ll|ll}
\hline Bond & Substitution & Angle & Substitution \\
\hline & & & \\
$\mathrm{CO}$ & $1.410 \mathrm{~B}$ & $\mathrm{COC}$ & $111.7 \mathrm{~B}$ \\
$\mathrm{CH}_{2}$ & $1.100 \mathrm{~B}$ & $\mathrm{OCH}_{\mathrm{s}}$ & $110.8 \mathrm{~B}$ \\
$\mathrm{CH}_{s}$ & $1.091 \mathrm{C}$ & $\mathrm{OCH}_{\mathrm{s}}$ & $107.2 \mathrm{C}$ \\
& & $\mathrm{H}_{\mathrm{a}} \mathrm{CH}_{\mathrm{a}}$ & $108.7 \mathrm{~B}$ \\
& & $\mathrm{H}_{\mathrm{a}} \mathrm{CH}_{\mathrm{s}}$ & $109.5 \mathrm{C}$
\end{tabular}

Hydrogens $s$ and a are in and out of the heavy atom plane, re. spectively.

[l] U. Blukis, P. H. Kasai, and R. J. Myers, J. Chem. Phys. 38, 2753 (1963).

\section{Methyl Sulfoxide}

\begin{tabular}{cc|cr}
$\mathrm{C}_{2} \mathrm{H}_{n} \mathrm{OS}$ & & $\left(\mathrm{CH}_{3}\right)_{2} \mathrm{SO}$ & $\mathrm{C}_{5}$ \\
\hline \hline Bond & Effective & Angle & Effective \\
\hline & & $\mathrm{CSC}$ & $96.4 \mathrm{X}$ \\
$\mathrm{CS}$ & $1.810 \mathrm{X}$ & $\mathrm{SCH}$ & $107.5 \mathrm{X}$ \\
$\mathrm{SO}$ & $1.477 \mathrm{X}$ & $\mathrm{OSC}$ & $106.7 \mathrm{X}$ \\
$\mathrm{CH}$ & $1.095 \mathrm{X}$ & & \\
\hline
\end{tabular}

Methyl groups were assumed to be symmetric with $C_{3}$ axis along the CS bond.

[1] H. Dreizler and G. Dendl, Z. Naturforsch. 19a, 512 (1964).

\section{gauche-Ethanethiol}

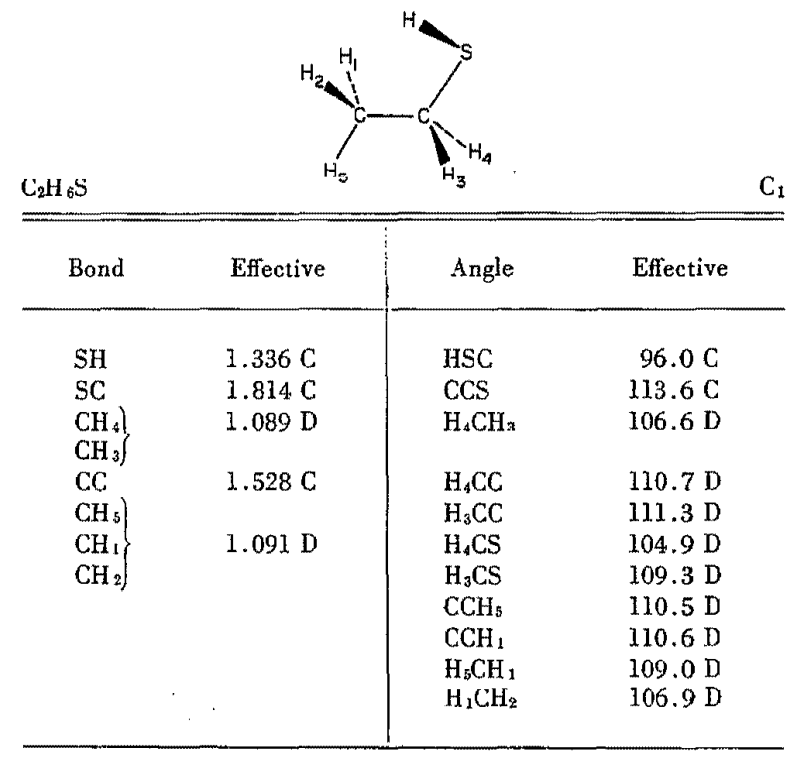

Assumed: $\mathrm{CH}_{3}=\mathrm{CH}_{4}, \mathrm{CH}_{1}=\mathrm{CH}_{2}, \mathrm{CH}_{5}=\mathrm{CH}_{1}$, and $\mathrm{H}_{5} \mathrm{H}_{1}=$ $\mathrm{H}_{3} \mathrm{H}_{2}$.

[1] J. Nakagawa, K. Kuwada, and M. Hayashi, Bull. Chem. Soc. Japan 49, 3420 (1976).

[2] R. E. Schmidt and C. R. Quade, J. Chem. Phys. 62, 864 (1975).

[3] M. Hayashi, J. Nakagawa, and K. Kuwada, Chem. Lett. 1975, 1267 (1975). 


\section{frans-Ethanethiol}

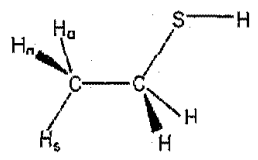

$\mathrm{C}_{8} \mathrm{H}_{4} \mathrm{~S}$

\begin{tabular}{ll|ll}
\hline Bond & Substitution & Angle & Substitution \\
\hline $\mathrm{SH}$ & $1.322 \mathrm{C}$ & $\mathrm{CSH}$ & $96.2 \mathrm{~B}$ \\
$\mathrm{SC}$ & $1.820 \mathrm{~B}$ & $\mathrm{CCS}$ & $108.6 \mathrm{~B}$ \\
$\mathrm{CC}$ & $1.529 \mathrm{~B}$ & $\mathrm{SCH}$ & $109.4 \mathrm{~B}$ \\
$\mathrm{CH}$ & $1.090 \mathrm{~B}$ & $\mathrm{CCH}$ & $110.2 \mathrm{D}$ \\
$\mathrm{CH}_{\mathrm{a}}$ & $1.092 \mathrm{C}$ & $\mathrm{HCH}$ & $108.9 \mathrm{~B}$ \\
$\mathrm{CH}_{\mathrm{s}}$ & $1.095 \mathrm{C}$ & $\mathrm{CCH}$ & $109.7 \mathrm{C}$ \\
& & $\mathrm{CCH}_{\mathrm{a}}$ & $110.6 \mathrm{~B}$ \\
& & $\mathrm{H}_{\mathrm{B}} \mathrm{CH}_{3}$ & $108.9 \mathrm{C}$ \\
& & $\mathrm{H}_{\mathrm{s}} \mathrm{CH}_{\mathrm{a}}$ & $108.1 \mathrm{C}$ \\
\hline
\end{tabular}

[1] M. Hayashi, H. Imaishi, and K. Kuwada, Bull. Chem. Soc. Japan 47, 2382 (1974).

[2] M. Hayashi, H. Imaishi, K. Ohno, and H. Murata, Bull. Chem. Soc. Japan 44, 872 (1971).

[3] Ch. 0. Kadzhar, A. A. Abbasov, and L. M. Imanov, Opt. Spectrosc. (USSR) 24, 334 (1968).

[4] R. E. Schmidt and C. R. Quade, Bull. Amer. Phys. Soc. II 17, 657 (1972).

Thiobismethane

(Dimethyl sulfide)

$\mathrm{C}_{2} \mathrm{H}_{6} \mathrm{~S}$

$\mathrm{CH}_{3} \mathrm{SCH}_{3}$

\begin{tabular}{ll|lc}
\hline \hline \multirow{2}{*}{ Bond } & Substitution & Angle & Substitution \\
\hline & & & \\
$\mathrm{CS}$ & $1.802 \mathrm{~A}$ & $\mathrm{CSC}$ & $98.87 \mathrm{~A}$ \\
$\mathrm{CH}_{\mathrm{a}}$ & $1.091 \mathrm{~A}$ & $\mathrm{H}_{\mathrm{a}} \mathrm{CH}_{\mathrm{a}}$ & $109.5 \mathrm{~A}$ \\
$\mathrm{CH}_{\mathrm{a}}$ & $1.091 \mathrm{E}$ & $\mathrm{H}_{\mathrm{a}} \mathrm{CH}_{\mathrm{s}}$ & $109.6 \mathrm{E}$ \\
& & $\mathrm{SCH}_{\mathrm{s}}$ & $110.75 \mathrm{~A}$ \\
& & $\mathrm{SCH}_{\mathrm{s}}$ & $106.62 \mathrm{E}$ \\
& & \\
\hline
\end{tabular}

$s$ and a refer to methyl protons in and out of the plane of sym inetry, reepectively.

[1] L. Pierce and M. Hayashi, J. Chem. Phys. 35, 479 (1961).

[2] H. Dreizler and H. D. Rudolph, Z. Naturforsch. 17a, 712 (1962).

\section{Dimethyl Disulfide}

$\mathrm{C}_{2} \mathrm{H}_{6} \mathrm{~S}_{2}$

$\mathrm{CH}_{3} \mathrm{SSCH}_{3}$

$\mathrm{C}_{2}$

\begin{tabular}{cc|cc}
\hline Bond & Effective & Angle & Effective \\
\hline SS & $2.038 \mathrm{X}$ & $\mathrm{SSC}$ & $102.8 \mathrm{X}$ \\
SC & $1.810 \mathrm{X}$ & $\mathrm{SCH}$ & $108.9 \mathrm{X}$ \\
$\mathrm{CH}$ & $1.097 \mathrm{X}$ & $\delta^{\mathrm{a}}$ & $84.7 \mathrm{X}$ \\
\hline
\end{tabular}

Methyl groups were assumed to be symmetric with $C_{3}$ axis along C-S bond.

a $\delta$ is the dihedral angle between the two SSC planes.

[1] D. Sutter, H. Driezler and H. D. Rudolph, Z. Naturforsch. 20a, 1676 (1965).

transw Ethane Selenol

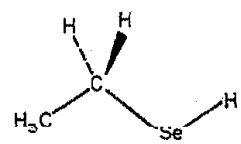

$\mathrm{C}_{2} \mathrm{H}_{6} \mathrm{Se}$

\begin{tabular}{lrr|cr}
\hline Bond & Substitution & Effective & Angle & Effective \\
CC & & $1.543 \mathrm{C}$ & CCSe & $108.8 \mathrm{C}$ \\
$\mathrm{CSc}$ & & $1.950 \mathrm{C}$ & CSeII & $93.3 \mathrm{C}$ \\
$\mathrm{SeH}$ & $1.444 \mathrm{C}$ & & & \\
\hline
\end{tabular}

All $\mathrm{CH}$ parameters were assumed. A more stable gauche form was observed also, with a dihedral angle (measured from cis position) of $62.0^{\circ}(\mathrm{X})$.

[1] J. R. Durig and W. E. Bucy, J. Mol. Spectrosc. 64, 474 (1977).

\section{Methyl Selenide}

$\mathrm{C}_{2} \mathrm{H}_{6} \mathrm{Se} \quad \mathrm{CH}_{3} \mathrm{SeCH}_{3} \quad . \mathrm{C}_{2 \pi}$

\begin{tabular}{|c|c|c|c|c|c|}
\hline Bond & $\begin{array}{l}\text { Substitu- } \\
\text { tion }\end{array}$ & Effective & Angle & $\begin{array}{c}\text { Substitu- } \\
\text { tion }\end{array}$ & Effective \\
\hline $\begin{array}{l}\mathrm{SeC} \\
\mathrm{CH}\end{array}$ & $1.943 \mathrm{~B}$ & $1.093 \mathrm{C}$ & $\begin{array}{l}\mathrm{CSeC} \\
\mathrm{SeCH}_{s} \\
\mathrm{SeCH}_{.} \\
\mathrm{HCH}\end{array}$ & $96.2 \mathrm{~B}$ & $\begin{array}{l}109.6 \mathrm{C} \\
106.7 \mathrm{C} \\
110.3 \mathrm{C}\end{array}$ \\
\hline
\end{tabular}

Methyl groups assumed symmetric.

[1] J. F. Beecher, J. Mol. Spectrosc. 4, 414 (1966). 
Vinylsilane

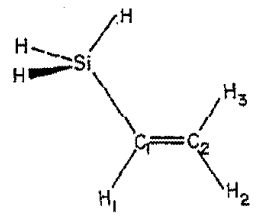

$\mathrm{C}_{2} \mathrm{H}_{6} \mathrm{Si}$

\begin{tabular}{|c|c|c|c|c|c|}
\hline Bond & $\begin{array}{l}\text { Substitu- } \\
\text { tion }\end{array}$ & Effective & Angle & $\begin{array}{l}\text { Substitu- } \\
\text { tion }\end{array}$ & Effective \\
\hline $\mathrm{CC}$ & $1.347 \mathrm{~B}$ & & SiCC & & $122.9 \mathrm{C}$ \\
\hline $\mathrm{SiC}$ & & $1.853 \mathrm{C}$ & $\mathrm{HSiH}$ & & $108.7 \mathrm{C}$ \\
\hline $\mathrm{SiH}$ & & $1.475 \mathrm{C}$ & $\mathrm{C}_{2} \mathrm{C}_{1} \mathrm{H}_{4}$ & $178.0 \mathrm{~B}$ & \\
\hline $\mathrm{C}_{1} \mathrm{H}_{x}$ & $1.094 \mathrm{~B}$ & & $\mathrm{C}_{1} \mathrm{C}_{2} \mathrm{H}_{2}$ & & $120.6 \mathrm{X}$ \\
\hline $\mathrm{C}_{2} \mathrm{H}_{3}$ & $1.097 \mathrm{~B}$ & & $\mathrm{C}_{2} \mathrm{C}_{2} \mathrm{H}_{3}$ & $120.3 \mathrm{~B}$ & \\
\hline
\end{tabular}

The $\mathrm{C}_{2} \mathrm{H}_{2}$ distance was assumed to be equal to the $\mathrm{C}_{2} \mathrm{H}_{3}$ distance. The $\mathrm{SiH}_{3}$ group was assumed to be symmetric. The axis of the $\mathrm{SiH}_{3}$ group is tilted $1.8^{\circ}$ toward the methylene group.

[1] J. M. O'Reilly and L. Yierce, J. Chem. Phys. 34, 1176 (1961).

\section{Dimethylzine}

\begin{tabular}{cccc}
$\mathrm{C}_{2} \mathrm{H}_{6} \mathrm{Zn}$ & \multicolumn{1}{c}{$\mathrm{CH}_{3} \mathrm{ZnCH}_{3}$} & Undetermined \\
\hline Bond & Effective & Angle & Effective \\
\hline $\mathrm{C}-\mathrm{Zn}$ & $1.929 \mathrm{C}$ & $\mathrm{HCH}$ & $107.7 \mathrm{X}$
\end{tabular}

In order to calculate the $\mathrm{HCH}$ angle it was necessary to assume the $\mathrm{C}-\mathrm{H}$ bond distance; the uncertainty in the angle is therefore impossible to evaluate.

[1] K. S. Rao, B. P. Stoicheff and R. Turner, Can. J. Phys. 38, 1516 (1960).

\section{2,4-Dicarbaheptaborane (7)}

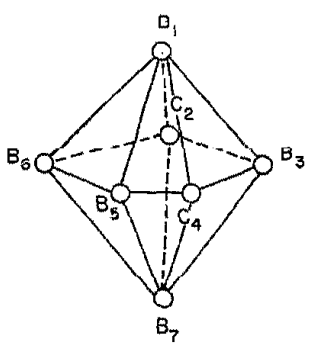

$\mathrm{C}_{2} \mathrm{H}_{7} \mathrm{~B}_{5}$

$\mathrm{C}_{2 \mathrm{v}}$

\begin{tabular}{cc|cc}
\hline Bond & Substitution & Angle & Substitution \\
\hline & & & \\
$\mathrm{C}_{2} \mathrm{~B}_{6}$ & $1.563 \mathrm{~B}$ & $\mathrm{C}_{2} \mathrm{~B}_{3} \mathrm{C}_{4}$ & $99.9 \mathrm{~B}$ \\
$\mathrm{C}_{2} \mathrm{~B}_{3}$ & $1.546 \mathrm{~B}$ & $\mathrm{~B}_{3} \mathrm{C}_{4} \mathrm{~B}_{3}$ & $116.8 \mathrm{~A}$ \\
$\mathrm{C}_{2} \mathrm{~B}_{3}$ & $1.708 \mathrm{~B}$ & $\mathrm{C}_{4} \mathrm{~B}_{5} \mathrm{~B}_{6}$ & $103.2 \mathrm{~A}$ \\
$\mathrm{~B}_{3} \mathrm{~B}_{6}$ & $1.651 \mathrm{~B}$ & $\mathrm{C}_{2} \mathrm{~B}_{3} \mathrm{~B}_{7}$ & $79.7 \mathrm{C}$ \\
$\mathrm{B}_{3} \mathrm{~B}_{5}$ & $1.815 \mathrm{D}$ & & \\
$\mathrm{B}_{1} \mathrm{~B}_{3}$ & $1.818 \mathrm{D}$ & & \\
& & & \\
\hline
\end{tabular}

In above figure, one hydrogen is bonded to each heavy atom.

[1] R. A. Beaudet and R. L. Poynter, J. Chem. Phys. 43, 2166 (1965).

[2] R. A. Beaudet and R. L. Poynter, J. Amer. Chem. Soc. 86, 1258 (1964).

trans-Chloroethylsilane

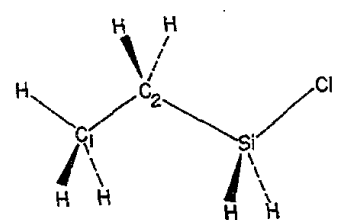

$\mathrm{C}_{2} \mathrm{H}_{7} \mathrm{CISi}$

\begin{tabular}{ll|ll}
\hline Bond & Effective & Angle & Effective \\
\hline $\mathrm{SiCl}$ & $2.060 \mathrm{X}$ & $\mathrm{C}_{2} \mathrm{SiCl}$ & $109.9 \mathrm{X}$ \\
$\mathrm{SiH}$ & $1.478 \mathrm{X}$ & & \\
$\mathrm{SiC}_{2}$ & $1.869 \mathrm{X}$ & $\mathrm{C}_{2} \mathrm{SiH}$ & $113.6 \mathrm{X}$ \\
$\mathrm{C}_{1} \mathrm{C}_{2}$ & $1.532 \mathrm{X}$ & $\mathrm{SiC}_{2} \mathrm{H}$ & $105.6 \mathrm{X}$ \\
$\mathrm{C}_{2} \mathrm{H}$ & $1.107 \mathrm{X}$ & $\mathrm{C}_{2} \mathrm{C}_{1} \mathrm{H}$ & $111.9 \mathrm{X}$ \\
$\mathrm{C}_{1} \mathrm{H}$ & $1.082 \mathrm{X}$ & $\mathrm{C}_{1} \mathrm{C}_{2} \mathrm{Si}$ & $111.3 \mathrm{X}$ \\
& & $\alpha_{1}$ & $117.7 \mathrm{X}$ \\
& & $\alpha_{2}$ & $121.8 \mathrm{X}$ \\
\hline
\end{tabular}

$\alpha_{1}$ is the angle between the CCSi plane and the CSiH plane and $\alpha_{2}$ is the angle between the CCSi plane and the $\mathrm{SiCH}$ plane. A gauche form exists aiso, with $\mathrm{C}_{1} \mathrm{C}_{2} \mathrm{Si}=113.0(\mathrm{X})$ and the $\mathrm{SiH}_{2} \mathrm{Cl}$ group rotated by $120.4^{\circ}(\mathrm{X})$

[I] V. Typke, M. Dakkouri and W. Zeil, Z. Naturforsch. 29a, $1081(1974)$ 


\section{Dimethylamine}

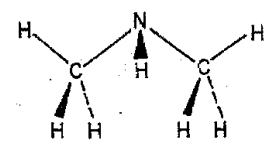

$\mathrm{C}_{2} \mathrm{H}_{7} \mathrm{~N}$

\begin{tabular}{ll|ll}
\hline Bond & Substitution & Angle & Substitution \\
\hline & & & \\
$\mathrm{CN}$ & $1.464 \mathrm{~B}$ & $\mathrm{CNC}$ & $112.0 \mathrm{~A}$ \\
$\mathrm{NH}$ & $1.022 \mathrm{C}$ & $\mathrm{HCH}$ (ave) & $108.5 \mathrm{C}$ \\
$\mathrm{CH}$ (ave) & $1.090 \mathrm{C}$ & $\mathrm{HNC}$ & $108.6 \mathrm{~B}$
\end{tabular}

Within experimental error, the $\mathrm{CH}$ bond is found to lie in the CNC plane.

[1] J. E. Wollrab and V. W. Laurie, J. Chem. Phys. 48, 5058 (1968).

\begin{tabular}{lll|lll} 
& & & \\
& & & & \\
\hline
\end{tabular}

$\mathrm{OH} \cdot \mathrm{N} \mathrm{N}$ hydrogen bond was very sensitive to isotopic substitution of $\mathrm{D}$ for $\mathrm{H}$ and, consequently, structural quality is diminished. $\mathrm{CH}$ parameters were assumed.

- Dihedral angles between indicated planes.

[1] R. E. Penn and R. F. Curl, J. Chem. Phys. 55, 651 (1971).

[2] R. E. Penn and R. J. Olsen, J. Mol. Spectrosc. 62, 423 (1976).

\section{Ethylphosphine}

$\mathrm{C}_{2} \mathrm{H}_{7} \mathrm{P}$

$\mathrm{CH}_{3} \mathrm{CH}_{2} \mathrm{PH}_{2}$

Microwave data shows the presence of trans and gauche isomers, with the trans isomer more stable by $200 \mathrm{~cm}^{-1}$.

C $\quad$ [1] J. R. Durig and A. W. Cox, J. Chem. Phys. 64, 1930 (1976).

\section{Dimethylphosphine}

\begin{tabular}{cc|cc}
$\mathrm{C}_{2} \mathrm{H}_{7} \mathrm{P}$ & $\left(\mathrm{CH}_{3}\right)_{2} \mathrm{PH}$ & $\mathrm{C}_{8}$ \\
\hline \hline Bond & Effective & Angle & Effective \\
\hline $\mathrm{CP}$ & $1.848 \mathrm{X}$ & $\mathrm{CPC}$ & $99.7 \mathrm{X}$ \\
$\mathrm{PH}$ & $1.419 \mathrm{X}$ & $\mathrm{CPH}$ & $97.0 \mathrm{X}$
\end{tabular}

The methyl group was assumed to be symmetric with $\mathrm{CH}=$ 1.093 and $\mathrm{HCH}=108.8^{\circ}$. The axis of the methyl group was assumed to be titled outward from the $\mathrm{CP}$ axis by $1^{\circ}$. The $\mathrm{CH}_{3}$ group was assumed to be staggered with respect to the $\mathrm{PH}$ bond.

[1] R. Nelson, J. Chem. Phys, 39, 2382 (1963).

\section{1,7-Dicarba-closo-octaborane (8)}

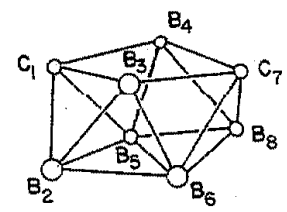

$\mathrm{C}_{2} \mathrm{H}_{8} \mathrm{~B}_{6}$

\begin{tabular}{ll|ll}
\hline Bond & Substitution & Angle & Substitution \\
\hline & $1.813 \mathrm{C}$ & & \\
$\mathrm{B}_{2} \mathrm{~B}_{3}$ & $1.843 \mathrm{C}$ & $\mathrm{B}_{2} \mathrm{~B}_{3} \mathrm{~B}_{6}$ & $52.7 \mathrm{~B}$ \\
$\mathrm{~B}_{2} \mathrm{~B}_{5}$ & $\mathrm{~B}_{5} \mathrm{~B}_{2} \mathrm{~B}_{6}$ & $66.9 \mathrm{C}$ \\
$\mathrm{B}_{2} \mathrm{~B}_{6}$ & $1.685 \mathrm{C}$ & $\mathrm{B}_{2} \mathrm{~B}_{6} \mathrm{~B}_{5}$ & $60.4 \mathrm{C}$ \\
$\mathrm{B}_{3} \mathrm{~B}_{4}$ & $1.886 \mathrm{C}$ & $\mathrm{B}_{2} \mathrm{~B}_{3} \mathrm{~B}_{6}$ & $54.3 \mathrm{~B}$ \\
$\mathrm{~B}_{3} \mathrm{~B}_{6}$ & $1.880 \mathrm{C}$ & $\mathrm{B}_{2} \mathrm{~B}_{6} \mathrm{~B}_{3}$ & $60.8 \mathrm{~B}$ \\
$\mathrm{~B}_{3} \mathrm{~B}_{6}$ & $1.949 \mathrm{C}$ & $\mathrm{B}_{3} \mathrm{~B}_{3} \mathrm{~B}_{6}$ & $64.9 \mathrm{~B}$ \\
\hline
\end{tabular}

[1] H. N. Rogers, K. Lau and R. A. Beaudet, Inorg. Chem. 15, 1775 (1976).

\section{Dimethylgermane}

\begin{tabular}{cc|cc}
$\mathrm{C}_{2} \mathrm{H}_{8} \mathrm{Ge}$ & $\left(\mathrm{CH}_{3}\right)_{2} \mathrm{GeH}_{2}$ & $\mathrm{C}$ \\
\hline Bond & Effective & Angle & Effective \\
\hline $\mathrm{GeC}$ & $1.95 \mathrm{C}$ & $\mathrm{CGeG}$ & $110 \mathrm{C}$ \\
\hline
\end{tabular}

$\mathrm{CH}$ and $\mathrm{HCH}$ parameters assumed from $\mathrm{CH}_{3} \mathrm{GeH}_{3}$.

[1] E. C. Thomas and V. W. Laurie, J. Chem. Phys. 50, 3512 (1969). 
Allyblomomo

\begin{tabular}{|c|c|c|c|}
\hline $11,4, i$ & $11_{3} \mathrm{O}$ & ${ }_{2} \mathrm{GeH}_{3}$ & \\
\hline Bond & Effective & Angle & Effective \\
\hline $\mathrm{GeCl}$ & $1.949 \mathrm{C}$ & $\mathrm{GeCC}$ & $112.2 \mathrm{C}$ \\
\hline CC & $1.545 \mathrm{C}$ & $\mathrm{HGeH}$ & $108.6 \mathrm{C}$ \\
\hline $\mathrm{GeH}$ & $1.522 \mathrm{C}$ & $\mathrm{HGeC}$ & $109.7 \mathrm{C}$ \\
\hline $\mathrm{C}^{(2)} \mathrm{H}$ & $1.093 \mathrm{C}$ & $\mathrm{HC}^{(2)} \mathrm{H}$ & $106.4 \mathrm{C}$ \\
\hline \multirow[t]{3}{*}{$\mathrm{C}^{(1)} \mathrm{H}$} & $1.091 \mathrm{C}$ & $\mathrm{GeC}^{(2)} \mathrm{H}$ & $111.6 \mathrm{C}$ \\
\hline & & $\mathrm{C}^{(9)} \mathrm{C}^{(1)} \mathrm{H}$ & $110.9 \mathrm{C}$ \\
\hline & & $\mathrm{HC}^{(1)} \mathrm{H}$ & $108.0 \mathrm{C}$ \\
\hline
\end{tabular}

Methyl and germyl groups were assumed to be symmetrical.

[1] J. R. During, A. D. Lopata and P. Groner, J. Chem. Phys. 66, 1888 (1977)

\section{Dimethylsilane}

\begin{tabular}{cccc}
$\mathrm{C}_{2} \mathrm{H}_{\mathrm{B}} \mathrm{Si}$ & $\left(\mathrm{CH}_{3}\right)_{2} \mathrm{SiH}_{2}$ & $\mathrm{C}_{2 \mathrm{v}}$ \\
\hline Bond & Substitution & Angle & Substitution \\
\hline $\mathrm{SiC}$ & $1.867 \mathrm{~A}$ & $\mathrm{CSiC}$ & $110.98 \mathrm{~A}$ \\
$\mathrm{SiH}$ & $1.483 \mathrm{~B}$ & $\mathrm{HSiH}$ & $107.83 \mathrm{~B}$ \\
$\mathrm{CH}$ & $1.095 \mathrm{~B}$ & $\mathrm{HCH}$ & $108.00 \mathrm{~B}$ \\
& & $\theta^{\mathrm{a}}$ & $110.83 \mathrm{~B}$ \\
\hline
\end{tabular}

Methyl groups assumed to be symmetric.

a $\theta=$ angle between the symmetry axes of the two methyl groups. [1] L. Pierce, J. Chem. Phys. 34, 498 (1961).

\section{1,6-Dicarbanonaborane (9)}

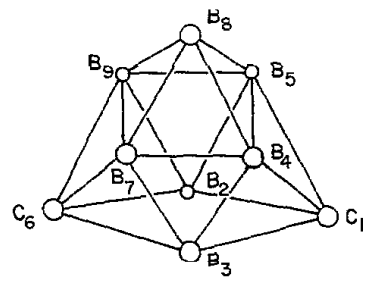

$\mathrm{C}_{2} \mathrm{H}_{3} \mathrm{~B}_{7}$

\begin{tabular}{|c|c|}
\hline Bond & Substitution \\
\hline $\mathrm{B}_{8} \mathrm{~B}_{9}$ & $1.712 \mathrm{C}$ \\
\hline $\mathrm{B}_{5} \mathrm{~B}_{9}$ & $1.995 \mathrm{~B}$ \\
\hline $\mathrm{B}_{3} \mathrm{~B}_{4}$ & $1.976 \mathrm{C}$ \\
\hline $\mathrm{B}_{7} \mathrm{~B}_{9}$ & $1.784 \mathrm{~B}$ \\
\hline $\mathrm{B}_{2} \mathrm{~B}_{3}$ & $1.805 \mathrm{~B}$ \\
\hline
\end{tabular}

[1] K. Lau and R. A. Beaudet, Inorg. Chem. 15, 1059 (1976).
Dimethylphosphine-borane

\begin{tabular}{|c|c|c|c|c|c|}
\hline $\mathrm{C}_{2} \mathrm{H}_{10} \mathrm{BP}$ & & & & & $\mathrm{C}_{5}$ \\
\hline Bond & $\begin{array}{l}\text { Substitu- } \\
\text { tion }\end{array}$ & Effective & Angle & $\begin{array}{l}\text { Substitu- } \\
\text { tion }\end{array}$ & Effective \\
\hline $\mathrm{BH}_{\mathrm{a}}$ & $1.216 \mathrm{C}$ & & $\mathrm{PBH}_{\mathrm{s}}$ & $104.8 \mathrm{C}$ & \\
\hline $\mathrm{BH}$. & $1.212 \mathrm{C}$ & & $\mathrm{PBH}$. & $104.9 \mathrm{C}$ & \\
\hline $\mathrm{PB}$ & & $1.898 \mathrm{D}$ & $\mathrm{CPB}$ & & $114.6 \mathrm{D}$ \\
\hline PC & & $1.813 \mathrm{D}$ & $\mathrm{CPC}$ & & $105.4 \mathrm{D}$ \\
\hline PH & & 1.4.14 D & $\mathrm{BPH}$ & & $118.1 \times$ \\
\hline
\end{tabular}

Methyl group parameters were assumed and borane group was assumed to be symmetrical about the PB bond.

[1] J. F. Durig, B. A. Hudgens, Y. S. Li and J. D. Odom, J. Chem. Phys. 61, 4390 (1974).

\section{$\mu$-(Dimethylamino)diborane (6) \\ (N,N-Dimethylaminodiborane)}

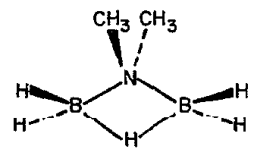

\begin{tabular}{|c|c|c|c|c|c|}
\hline Bond & $\begin{array}{l}\text { Substitu- } \\
\text { tion }\end{array}$ & Effective & Angle & $\begin{array}{l}\text { Substitu- } \\
\text { tion }\end{array}$ & Effective \\
\hline BB & $1.916 \mathrm{~B}$ & & $\mathrm{H}_{t} \mathrm{BH}_{\mathrm{t}}$ & $119.6 \mathrm{C}$ & \\
\hline $\mathrm{BH}_{\mathrm{t}}$ & $1.191 \mathrm{C}$ & & $\mathrm{BH}_{\mathrm{br}} \mathrm{B}$ & $89.1 \mathrm{C}$ & \\
\hline $\mathrm{BH}_{\mathrm{br}}$ & $1.365 \mathrm{C}$ & & BNB $^{\mathbf{A}}$ & & $76.8 \mathrm{X}$ \\
\hline $\mathrm{BN}^{\mathrm{a}}$ & & $1.544 \mathrm{X}$ & $\mathrm{CNC}^{s}$ & & $110.0 \times$ \\
\hline $\mathrm{CN}^{\star}$ & & $1.488 \mathrm{X}$ & $\epsilon^{b}$ & $16.7 \mathrm{C}$ & \\
\hline
\end{tabular}

$H_{t}$ and $H_{b r}$ refer to the terminal and bridge hydrogens, respectively.

- Obtained by assuming methyl group parameters.

b Angle made by $\mathrm{BH}_{2}$ plane and the plane perpendicular to the $\mathrm{C}_{2}$ symmetry axis.

[1] E. A. Cohen and R. A. Beaudet, Inorg. Chem. 12, 1570 (1973). 
Bromochloroacetylene

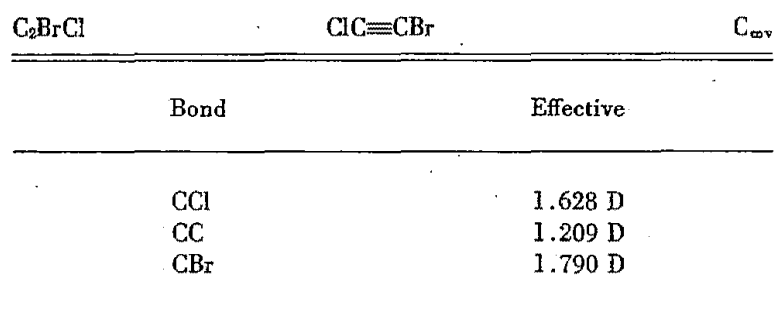

Ground state spectrum not observed. Rotational constants extra polated by observing several excited vibrational states.

[1] A. Bjørseth, E. Kloster-Jensen, K. M. Marstokk, and H. Møllendal, J. Mol. Struct. 6, 181 (1970).

\section{Isocyanomethylidyne}

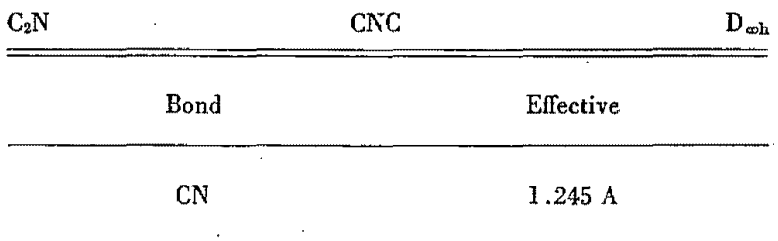

Ground electronic state is ${ }^{2} \mathrm{I}_{\mathrm{g}}$.

[1] A. J. Merer and D. N. Travis, Can. J. Phys. 44, 353 (1966).

\begin{tabular}{lccc}
\multicolumn{3}{c}{ Cyanomethylidyne } & $\mathrm{C}_{\infty \mathrm{v}}$ \\
\hline $\mathrm{C} \mathrm{C}_{2} \mathrm{~N}$ & $\mathrm{CCN}$ & Effective \\
\hline $\mathrm{C} \cdots \mathrm{N}$ & $2.56 \mathrm{D}$
\end{tabular}

Ground electronic state is ${ }^{2} \Pi_{\mathrm{r}}$.

[1] A. J. Merer and D. N. Travis, Can. J. Phys. 43, 1795 (1965).

\section{Cyanogen}

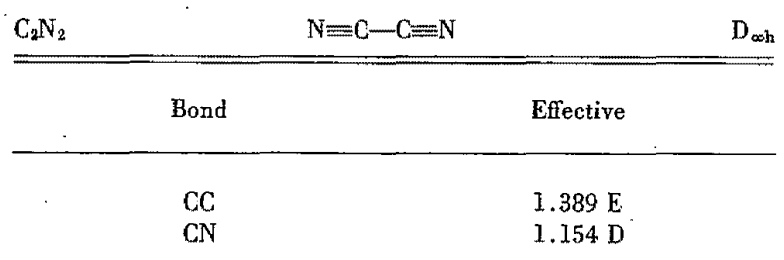

[1] A. G. Maki, J. Chem. Phys. 43, 3193 (1965).

\section{Cyanogen Isocyanate}

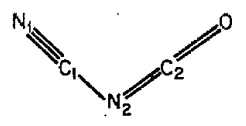

\begin{tabular}{ll|ll}
$\mathrm{C}_{2} \mathrm{~N}_{2} \mathrm{O}$ & & & \\
\hline \hline Bond & Effective & Angle & Effective. \\
\hline & & & \\
$\mathrm{N}_{1} \mathrm{C}_{2}$ & $1.164^{\mathrm{a}}$ & $\mathrm{NCN}$ & $180^{\mathrm{a}}$ \\
$\mathrm{N}_{2} \mathrm{C}_{2}$ & $1.218^{\mathrm{a}}$ & $\mathrm{NCO}$ & $180^{\mathrm{a}}$ \\
$\mathrm{CO}$ & $1.165^{\mathrm{a}}$ & $\mathrm{CNC}$ & $140 \mathrm{X}$ \\
$\mathrm{C}_{1} \mathrm{~N}_{2}$ & $1.283 \mathrm{X}$ & & \\
\hline
\end{tabular}

a Assumed values.

[1] W. H. Hocking and M. C. L. Gerry, J. Mol. Spectrose. 59, 338 (1976).

\section{Sulfur Cyanide}

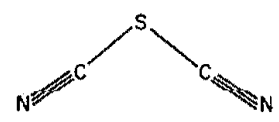

\begin{tabular}{ll|ll}
$\mathrm{C}_{2} \mathrm{~N}_{2} \mathrm{~S}$ & & $\mathrm{C}_{2 \mathrm{v}}$ \\
\hline Bond & Substitution & Angle & Substitution \\
\hline & & & \\
$\mathrm{SC}$ & $1.701 \mathrm{~B}$ & $\mathrm{CSC}$ & $98.3 \mathrm{~B}$ \\
$\mathrm{CN}$ & $1.156 \mathrm{~B}$ & $\mathrm{SCN}$ & $175.0 \mathrm{C}$
\end{tabular}

The SCN angle is such that the angle between the two $\mathrm{C} \equiv \mathrm{N}$ bond lines $\left(108.3^{\circ}\right)$ is Jarger than the CSC angle.

[1] L. Pierce, R. Nelson, and C. Thomas, J. Chem. Phys. 43, 3423 (1965).

\section{Dicarbon Monoxide}

\begin{tabular}{ccc}
$\mathrm{C}_{2} \mathrm{O}$ & $\mathrm{CCO}$ & $\mathrm{C}_{\infty \mathrm{r}}$ \\
\hline Bond & Effective \\
\hline $\mathrm{C} \cdots \mathrm{O}$ & $2.52 \mathrm{D}$
\end{tabular}

Ground olectrouic state is ${ }^{2} \mathrm{z}-$.

[1] C. Devillers and D. A. Ramsay, Can. J. Phys. 49, 2839 (1971). 


\section{$C_{3}$ Molecules}

Tricarbon (Propadiene-1,3-diylidene)

$\frac{\text { Bond }}{=} \frac{\mathrm{CCC}}{\text { Effective }} \mathrm{D}_{\infty \mathrm{s}}$

[1] A. E. Douglas, Astrophys. J. 114, 466 (1951).

[2] L. Gausset, G. Herzberg, A. Lagerqvist and B. Rosen, Astrophys. J. 142, 45 (1965).

\section{Bromocyanoacetylene}

\begin{tabular}{|c|c|c|}
\hline $\mathrm{C}_{3} \mathrm{BrN}$ & & $\mathrm{C}_{\boldsymbol{\alpha \omega v}}$ \\
\hline & Bond & Substitution \\
\hline & $\mathrm{CBr}$ & $1.786 \mathrm{~B}$ \\
\hline & $\mathrm{C}^{1} \mathrm{C}^{2}$ & 1.204. B \\
\hline & $\mathrm{C}^{2} \mathrm{C}^{3}$ & $1.370 \mathrm{~A}$ \\
\hline & $C N$ & $1.159 \mathrm{~A}$ \\
\hline
\end{tabular}

[1] T'. Bjorvalten, J, Mol. Struet, 20, 75 (1974).

1-Chloro-3,3,3-Trifluoropropyne

\begin{tabular}{ccc}
$\mathrm{C}_{3} \mathrm{ClF}_{3}$ & $\mathrm{~F}_{3} \mathrm{C}^{1} \mathrm{C}^{2}=\mathrm{C}^{3} \mathrm{Cl}$ & $\mathrm{C}_{8 \mathrm{v}}$ \\
\hline Bond & Substitution & Effectives \\
\hline $\mathrm{C}^{3} \mathrm{Cl}$ & $1.629 \mathrm{C}$ & $1.627 \mathrm{C}$ \\
$\mathrm{C}^{1} \ldots \mathrm{C}^{3}$ & $2.647 \mathrm{~B}$ & \\
$\mathrm{C}^{3} \mathrm{C}^{2}$ & & $1.453 \mathrm{C}$ \\
$\mathrm{C}^{2} \mathrm{C}^{3}$ & & $1.199 \mathrm{C}$ \\
$\mathrm{C}^{1 \mathrm{~F}}$ & & $1.336 \mathrm{C}$
\end{tabular}

The FCF angle was assumed in arder to determine these parameters.

[1] A. Bjørseth and K. M. Marstokk, J. Mol. Struct. 13, 191 (1972).

\section{Chlorocyanoacelylene}

\begin{tabular}{ccc}
$\mathrm{C}_{3} \mathrm{ClN}$ & $\mathrm{Cl}-\mathrm{Cl}=\mathrm{C}^{2}-\mathrm{C}^{3}=\mathrm{N}$ & $\mathrm{C}_{\text {cov }}$ \\
\hline Bond & Substitution \\
\hline $\mathrm{CCl}$ & $1.625 \mathrm{~B}$ \\
$\mathrm{C}^{1} \mathrm{C}^{2}$ & $1.209 \mathrm{~B}$ \\
$\mathrm{C}^{2} \mathrm{C}^{3}$ & $1.369 \mathrm{~B}$ \\
$\mathrm{CN}$ & $1.160 \mathrm{~A}$
\end{tabular}

[1] T. Bjorvatten, J. Mol. Struct. 20, 75 (1974).

\section{3,3,3-Trifluoro-1..Propyne}

\begin{tabular}{lcc|cc}
$\mathrm{C}_{3} \mathrm{HF}_{3}$ & & $\mathrm{H}-\mathrm{C} \equiv \mathrm{C}-\mathrm{CF}_{3}$ & $\mathrm{C}_{37}$ \\
\hline \hline Bond & Substitution & Effective & Angle & Effective \\
\hline & & & & \\
$\mathrm{HC}$ & $1.056 \mathrm{~B}$ & & $107.5 \mathrm{X}$ \\
$\mathrm{C}=\mathrm{C}$ & $1.201 \mathrm{~A}$ & & \\
$\mathrm{C}-\mathrm{C}$ & & $1.464 \mathrm{X}$ & & \\
$\mathrm{CF}$ & & $1.335 \mathrm{X}$ & & \\
\hline
\end{tabular}

[1] N. N. Shoolery, R. G. Shulman, W. F. Sheehan, Jr., V. Schomaker, and D. M. Yost, J. Chem. Phys. 19, 1364 (1951).

\section{2-Propynenifrile}

\begin{tabular}{ccc}
$\mathrm{C}_{i} \mathrm{HN}$ & $\mathrm{HCCCN}$ & $\mathrm{C}_{\infty \mathrm{rr}}$ \\
\hline \hline Bond & Substitution & Effective \\
\hline $\mathrm{CH}$ & $1.058 \mathrm{~A}$ & $1.057 \mathrm{~A}$ \\
$\mathrm{C}=\mathrm{C}$ & $1.205 \mathrm{~A}$ & $1.203 \mathrm{~A}$ \\
$\mathrm{C}-\mathrm{C}$ & $1.378 \mathrm{~A}$ & $1.382 \mathrm{~A}$ \\
$\mathrm{CN}$ & $1.159 \mathrm{~A}$ & $1.157 \mathrm{~A}$
\end{tabular}

[1] A. A. Westenberg and E. B. Wilson, Jr., J. Am. Chem. Soc. 72,199 (1950).

[2] J. K. Tyler and J. Sheridan, Trane. Faraday Soc. 59, 2661 (1963).

\section{1, 1-Difluoroallene}

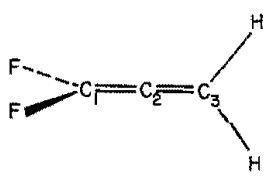

\section{$\mathrm{C}_{3} \mathrm{H}_{2} \mathrm{~F}_{2}$}

\begin{tabular}{llr|ccc}
\hline \hline Bond & Effective & $\begin{array}{c}\text { Substi- } \\
\text { tution }\end{array}$ & Angle & Effective & $\begin{array}{c}\text { Substi- } \\
\text { tution }\end{array}$ \\
\hline & & & & & \\
$\mathrm{C}_{2} \mathrm{C}_{3}$ & & $1.306 \mathrm{~B}$ & $\mathrm{HCH}$ & & $117.8 \mathrm{~B}$ \\
$\mathrm{CH}$ & & $1.086 \mathrm{~B}$ & $\mathrm{CCH}$ & & $121.1 \mathrm{~B}$ \\
$\mathrm{C}_{1} \mathrm{C}_{2}$ & $1.302 \mathrm{D}$ & & $\mathrm{FCF}$ & $110.2 \mathrm{D}$ & \\
$\mathrm{CF}$ & $1.323 \mathrm{D}$ & & $\mathrm{CCF}$ & $124.9 \mathrm{D}$ & \\
\hline [1] J. R. Durig, Y. S. Li, C. C. Tong, A. P. Zens and P. D. Ellis,
\end{tabular}
J. Am. Chem. Soc. 96, 3805 (1974). 
3,3-difluorocycloproperie

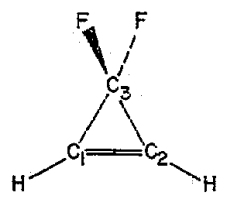

\begin{tabular}{lcc|ccc}
$\mathrm{C}_{3} \mathrm{H}_{2} \mathrm{~F}_{2}$ & & & $\mathrm{C}_{2 \mathrm{v}}$ \\
\hline Bond & $\begin{array}{c}\text { Substitu- } \\
\text { tion }\end{array}$ & Effective & Angle & $\begin{array}{c}\text { Substitu- } \\
\text { tion }\end{array}$ & Effective \\
\hline $\mathrm{C}_{1} \mathrm{C}_{2}$ & $1.321 \mathrm{~A}$ & & $\mathrm{FCF}$ & & $105.5 \mathrm{D}$ \\
$\mathrm{CH}$ & $1.075 \mathrm{~A}$ & & $\mathrm{C}_{1} \mathrm{C}_{3} \mathrm{C}_{2}$ & & $54.6 \mathrm{C}$ \\
$\mathrm{C}_{1} \mathrm{C}_{3}$ & & $1.438 \mathrm{C}$ & $\mathrm{HC}_{3} \mathrm{C}_{2}$ & $148.4 \mathrm{~A}$ & \\
$\mathrm{CF}$ & & $1.365 \mathrm{C}$ & & & \\
\end{tabular}

[1] K. R. Ramaprasad, V. W. Laurie, and N. C. Craig, J. Chem. Phys. 64, 4832 (1976).

\section{Malononitrile}

\begin{tabular}{cc|cc}
$\mathrm{C}_{3} \mathrm{H}_{2} \mathrm{~N}_{2}$ & $\mathrm{CH}_{2}(\mathrm{CN})_{2}$ & $\mathrm{C}_{20}$ \\
\hline Bond & Effective & Angle & Effective \\
\hline $\mathrm{CC}$ & $1.47 \mathrm{E}$ & $\mathrm{CCC}$ & $109 \mathrm{E}$ \\
$\mathrm{CN}$ & $1.17 \mathrm{E}$ & $\mathrm{HCH}$ & $109 \mathrm{E}$ \\
$\mathrm{CH}$ & $1.09 \mathrm{D}$ & $\mathrm{CCNa}$ & $1.74 \mathrm{E}$ \\
\hline
\end{tabular}

a The cyano group is bent outward.

[1] E. Hirota and Y. Morino, Bull. Chem. Soc. Japan 33, 705 (1960)

[2] E. Hirota and Y. Morino, Bull. Chem. Soc. Japan 33, 158 (1960).

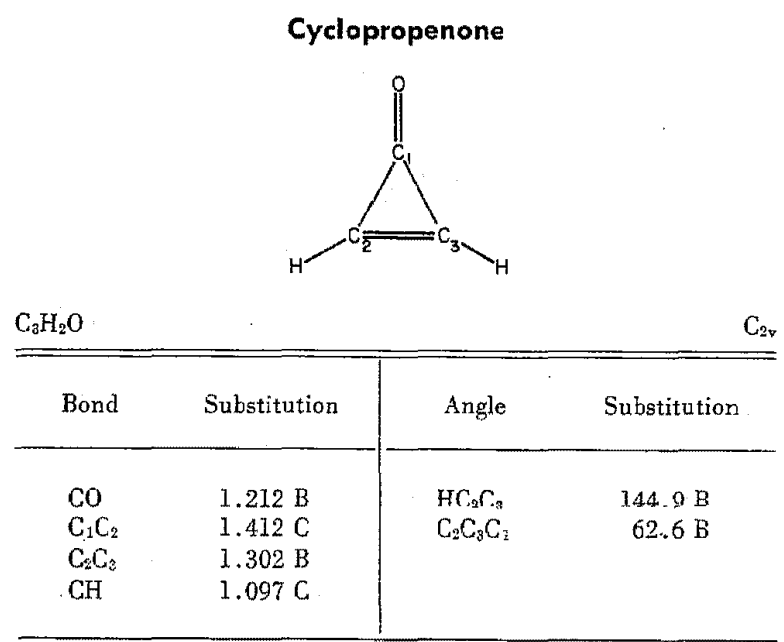

[1] R. C. Benson, W. H. Flygare, M. Oda and R. Breslow, J. Am. Chem Soc. 95, 2772 (1973).
2-Propynal

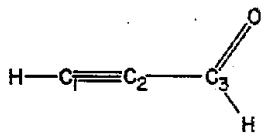

$\mathrm{C}_{3} \mathrm{H}_{2} \mathrm{O}$

$\mathrm{C}_{\mathrm{s}}$

\begin{tabular}{lcc|ccc}
\hline \hline Bond & $\begin{array}{c}\text { Substitu- } \\
\text { tion }\end{array}$ & Average & Angle & $\begin{array}{c}\text { Substitu- } \\
\text { tinn }\end{array}$ & Average \\
\hline $\mathrm{HC}_{1}$ & $1.055 \mathrm{~B}$ & $1.054 \mathrm{C}$ & $\mathrm{C}_{2} \mathrm{C}_{3} \mathrm{O}$ & $123.9 \mathrm{D}$ & $124.2 \mathrm{~B}$ \\
$\mathrm{HC}_{3}$ & $1.106 \mathrm{~B}$ & $1.114 \mathrm{C}$ & $\mathrm{C}_{2} \mathrm{C}_{3} \mathrm{H}$ & $113.9 \mathrm{D}$ & $113.8 \mathrm{D}$ \\
$\mathrm{C}_{1} \mathrm{C}_{2}$ & $1.209 \mathrm{~B}$ & $1.205 \mathrm{C}$ & $\mathrm{C}_{1} \mathrm{C}_{2} \mathrm{C}_{3}$ & $178.4 \mathrm{E}^{3}$ & $178.6 \mathrm{C}$ \\
$\mathrm{C}_{2} \mathrm{C}_{3}$ & $1.444 \mathrm{~B}$ & $1.449 \mathrm{~A}$ & $\mathrm{HC}_{2} \mathrm{C}_{2}$ & $180.0 \mathrm{E}$ & \\
$\mathrm{C}_{3} \mathrm{O}$ & $1.214 \mathrm{~B}$ & $1.212 \mathrm{~B}$ & & & \\
\hline
\end{tabular}

a The tilt is toward the aldehydic hydrogen.

[1] C. C. Costain and J. R. Morton, J. Chem. Phys. 31, 389 (1959).

[2] M. Sugie, T. Kukuyama and K. Kuchitsu, J. Mol. Struet. 14, 333 (1972)

\section{Propiolic Acid}

(Propargylic or Propynoic Acid)

$\mathrm{C}_{3} \mathrm{H}_{2} \mathrm{O}_{2}$

$\mathrm{HC} \equiv \mathrm{CCOOH}$

$\mathrm{C}_{\mathrm{a}}$

It was determined that the species is planar with the hydroxyl hydrogen cis to the carbonyl group.

[1] D. G. Lister and J. K. Tyler, Spectrochim. Acta 28A, 1423 (1972).

Cyclic Vinylene Ester (Vinylene Carbonate)

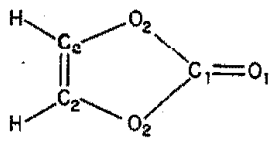

$\mathrm{C}_{3} \mathrm{Ir}_{2} \mathrm{O}_{3}$

$\mathrm{C}_{3 \mathrm{v}}$

\begin{tabular}{cc|cc}
\hline Bond & Substitution & Angle & Substitution \\
\hline & & & \\
\hline $\mathrm{C}_{2} \mathrm{C}_{2}$ & $1.331 \mathrm{~A}$ & $\mathrm{C}_{2} \mathrm{C}_{2} \mathrm{O}_{2}$ & $108.6 \mathrm{X}$ \\
$\mathrm{C}_{2} \mathrm{O}_{2}$ & $1.385 \mathrm{X}$ & $\mathrm{C}_{2} \mathrm{O}_{2} \mathrm{C}_{1}$ & $106.9 \mathrm{X}$ \\
$\mathrm{C}_{1} \mathrm{O}_{2}$ & $1.364 \mathrm{X}$ & $\mathrm{O}_{2} \mathrm{C}_{1} \mathrm{O}_{2}$ & $108.8 \mathrm{X}$ \\
$\mathrm{C}_{1} \mathrm{O}_{1}$ & $1.191 \mathrm{~A}$ & & \\
\hline
\end{tabular}

[1] W. F. White and J. E. Boggs, J. Chem. Phys. 54, 4714 (1971). 


\section{3-Bromopropyne \\ (Propargyl bromide)}

\begin{tabular}{|c|c|c|c|}
\hline $\mathrm{I}_{3} \mathrm{Br}$ & & $=\mathrm{C}^{1} \mathrm{H}$ & \\
\hline Bond & Effective & Angle & Effective \\
\hline $\begin{array}{l}\mathrm{C}^{3} \mathrm{Br} \\
\mathrm{C}^{2} \mathrm{C}^{3}\end{array}$ & $\begin{array}{l}1.94 \mathrm{E} \\
1.46 \mathrm{E}\end{array}$ & $\mathrm{C}^{2} \mathrm{C}^{3} \mathrm{~B} \mathrm{r}$ & $112 \mathrm{E}$ \\
\hline
\end{tabular}

Four isotopic species studied; $\mathrm{C}^{2} \mathrm{H}, \mathrm{C} \mathrm{CH}^{3} \mathrm{C}^{2} \equiv \mathrm{C}^{1}$ and $\mathrm{C}^{2} \mathrm{C}^{3} \mathrm{H}$ values were assumed.

[1] Y. Kikuchi, E. Hirota and Y. Morino, Bull. Chem. Soc. Japan 34, 348 (1961).

\section{3-Chloropropyne \\ (Propargyl Chloride)}

\begin{tabular}{cc|cc}
$\mathrm{C}_{3} \mathrm{H}_{3} \mathrm{Cl}$ & \multicolumn{2}{c}{$\mathrm{ClH}_{2} \mathrm{Cl}^{3} \mathrm{C}^{2}=\mathrm{C}^{1} \mathrm{H}$} & $\mathrm{C}_{8}$ \\
\hline \hline \multirow{2}{*}{ Bond } & Effective & Angle & Effective \\
\hline \multirow{2}{*}{$\mathrm{CCl}$} & $1.780 \mathrm{E}$ & $\mathrm{CCCl}$ & $111.9 \mathrm{E}$ \\
$\mathrm{C}^{2} \mathrm{C}^{3}$ & $1.465 \mathrm{E}$ & $\mathrm{HCH}$ & $108.7 \mathrm{D}$ \\
& & $\mathrm{C}^{2} \mathrm{C}^{3} \mathrm{H}$ & $111.5 \mathrm{E}$ \\
& &
\end{tabular}

Four isotopic species studied; $\mathrm{C}^{2} \mathrm{H}, \mathrm{C}^{3} \mathrm{H}, \mathrm{C}^{2} \equiv \mathrm{C}^{1}$ bond lengths were assumed.

[1] E. Hirota and Y. Morino, Bull. Chem. Soc. Japan 34, 341 (1961).
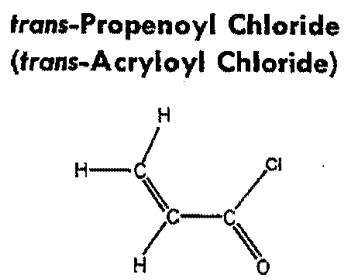

$\mathrm{C}_{3} \mathrm{H}_{3} \mathrm{ClO}$

Spectra were assigned for one isomer, established as the s-trans conformer as shown in the frgure. Evidence for a second conformer (cis or possibly gauche) was discussed but no assignment was obtained.

[1] R. Kewley, D. C. Hemphill, and R. F. Curl, Jr., J. Mol. Spectrosc. 44,443 (1972)

\section{cis-Propenoyl Fluoride \\ (cis-Acryloyl Fluoride)}

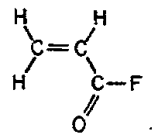

$\mathrm{C}_{3} \mathrm{H}_{3} \mathrm{FO}$

$\mathrm{C}_{\mathrm{s}}$

Existence of two planar rotational isomers was established. One isotopic species was assigned for each isomer and rotational constants were determined. Although the authors proposed structures consistent with rotational constants, they could not positively determine which isomer was cis and which was trans. Figure shows cis form; see acrylnyl chloride for trans form.

[1] J. J. Keirns and R. F. Curl, Jr., J. Chem. Phys. 48, 3773 (1968).

\section{cis-1,2,3-Trifuorocyclopropane}

\begin{tabular}{ccc|cc}
$\mathrm{C}_{3} \mathrm{H}_{3} \mathrm{~F}_{3}$ & \multicolumn{2}{c}{$\mathrm{HFC}-\mathrm{HFC}-\mathrm{HFC}$} & $\mathrm{C}_{3 \mathrm{~V}}$ \\
\hline \hline Bond & Substitution & Effective & Angle & Effective \\
\hline $\mathrm{CC}$ & $1.567 \mathrm{D}$ & & $\mathrm{HCF}$ & $112.3 \mathrm{~B}$ \\
$\mathrm{CH}$ & $1.095 \mathrm{~B}$ & & & \\
$\mathrm{CF}$ & & $1.354 \mathrm{~B}$ & & \\
\hline
\end{tabular}

[1] C. W. Gillies, J. Mol. Spectrosc. 59, 482 (1976).

\section{3,3,3-Trifluoro-1-propene}

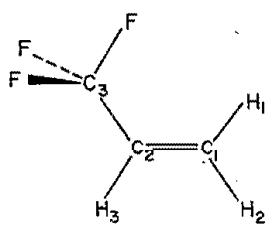

$\mathrm{C}_{*} \mathrm{H}_{3} \mathrm{~F}_{3}$

\begin{tabular}{ll|lr}
\hline Bond & Effective & Angle & Effective \\
\hline & & & \\
$\mathrm{C}_{2} \mathrm{C}_{3}$ & $1.489 \mathrm{~B}$ & $\mathrm{FCF}$ & $106.8 \mathrm{C}$ \\
$\mathrm{C}_{1} \mathrm{C}_{2}$ & $1.312 \mathrm{C}$ & $\mathrm{C}_{3} \mathrm{C}_{2} \mathrm{C}_{1}$ & $124.8 \mathrm{C}$ \\
$\mathrm{CH}_{1}$ & $1.085 \mathrm{C}$ & $\mathrm{CCH}_{1}$ & $120.6 \mathrm{D}$ \\
$\mathrm{CH}_{2}$ & $1.092 \mathrm{C}$ & $\mathrm{CCH}_{2}$ & $122.8 \mathrm{D}$ \\
$\mathrm{CH}_{3}$ & $1.109 \mathrm{~B}$ & $\mathrm{C}_{1} \mathrm{C}_{2} \mathrm{H}_{3}$ & $121.2 \mathrm{D}$ \\
$\mathrm{CF}^{\mathrm{a}}$ & $1.345 \mathrm{~B}$ & $\theta^{\mathrm{b}}$ & $1.0 \mathrm{D}$ \\
& & & \\
\hline
\end{tabular}

a Assumed value.

b Tilt angle of the $\mathrm{CF}_{3}$ symmetry axis from $\mathrm{C}-\mathrm{C}$ bond axis and away from the double bond.

[1] S. Saito and F. Makino, Bull. Chem. Soc., Japan 47, 1863 (1974). 
Trifluoroacelic Acid-Formic Acid Dlmer

\begin{tabular}{ccc}
$\mathrm{C}_{3} \mathrm{H}_{3} \mathrm{~F}_{3} \mathrm{O}_{4}$ & $\mathrm{CF}_{3} \mathrm{CO}_{2} \mathrm{H} \cdot \mathrm{HCO}_{2} \mathrm{H}$ & $\mathrm{C}_{8}$ \\
\hline Bond & Effective \\
\hline $0 \cdots 0$ & $2.67 \mathrm{X}$
\end{tabular}

[1] C. C. Costain and G. P. Srivastava, J. Chem. Phys. 41, 1620 (1964).

\section{Vinyl cyanide}

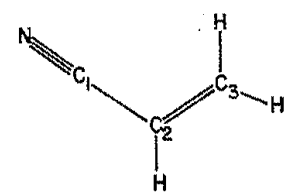

$\mathrm{C}_{3} \mathrm{H}_{8} \mathrm{~N}$

\begin{tabular}{ll|ll}
\hline \hline Bond & Substitution & Angle & Substitution \\
\cline { 3 - 4 } & & & \\
$\mathrm{CN}$ & $1.164 \mathrm{~B}$ & $\mathrm{C}_{1} \mathrm{C}_{2} \mathrm{C}_{3}$ & $122.6 \mathrm{C}$ \\
$\mathrm{C}_{1} \mathrm{C}_{2}$ & $1.426 \mathrm{~B}$ & $\mathrm{HC}_{2} \mathrm{C}_{3}$ & $121.7 \mathrm{~B}$ \\
$\mathrm{C}_{2} \mathrm{C}_{3}$ & $1.339 \mathrm{~B}$ & & \\
$\mathrm{C}_{2} \mathrm{H}$ & $1.086 \mathrm{~B}$ & & \\
\hline
\end{tabular}

$\mathrm{C}_{2} \mathrm{C}_{1} \mathrm{~N}$ was linear to within experimental accuracy.

[1] C. C. Costain and B. P. Stoicheff, J. Chem. Phys. 30, 777 (1959).

\section{Acetyl cyanide}

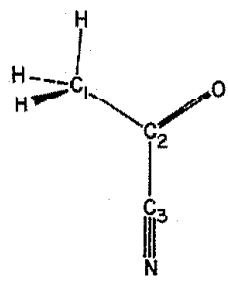

$\mathrm{C}_{5} \mathrm{H}_{3} \mathrm{NO}$

\begin{tabular}{lll|lll}
\hline \hline Bond Substitution & Effective & Angle & Substitution & Effective \\
\hline & & & & \\
$\mathrm{CO}$ & $1.226 \mathrm{D}$ & & $\mathrm{C}_{1} \mathrm{C}_{2} \mathrm{O}$ & $124.0 \mathrm{D}$ & \\
$\mathrm{C}_{2} \mathrm{C}_{2}$ & $1.490 \mathrm{D}$ & & $\mathrm{C}_{1} \mathrm{C}_{2} \mathrm{C}_{3}$ & $115.0 \mathrm{D}$ & \\
$\mathrm{C}_{2} \mathrm{C}_{3}$ & $1.466 \mathrm{C}$ & & $\mathrm{HCH}$ & & $108.7 \mathrm{C}$ \\
$\mathrm{CN}$ & $1.164 \mathrm{C}$ & & & & \\
$\mathrm{CH}$ & & $1.086 \mathrm{C}$ & & & \\
\hline
\end{tabular}

$\mathrm{C}_{2} \mathrm{C}_{3} \mathrm{~N}$ group was assumed to be linear, and the methyl group was assumed to be symmetrical.

[1] L. C. Krisher and E. B. Wilson, J. Chem. Phys., 31, 882 (1959)

$\mathrm{C}_{s}$
Isocyanoethene

(Vinyl isocyanate)

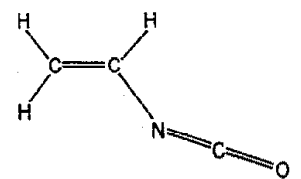

$\mathrm{C}_{3} \mathrm{H}_{3} \mathrm{NO}$

$\mathrm{C}_{\text {。 }}$

Microwave, data confirm the planar trans conformation of the molecule.

[1] A. Bouchy and G. Roussy, C. R. Acad. Sci. Paris 284, 411 (1977).

\section{Isoxazole}

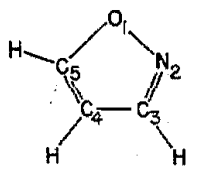

\begin{tabular}{cc|cc}
\hline \multicolumn{2}{c}{$\mathrm{C}_{3} \mathrm{H}_{3} \mathrm{NO}$} & & \\
\hline \hline Bond & Substitution & Angle & Substitution \\
\hline & & & \\
\hline $\mathrm{O}_{1} \mathrm{~N}_{2}$ & $1.399 \mathrm{~A}$ & $\mathrm{C}_{5} \mathrm{O}_{5} \mathrm{~N}_{2}$ & $108.9 \mathrm{~A}$ \\
$\mathrm{~N}_{2} \mathrm{C}_{3}$ & $1.308 \mathrm{~A}$ & $\mathrm{O}_{1} \mathrm{~N}_{2} \mathrm{C}_{3}$ & $105.3 \mathrm{~A}$ \\
$\mathrm{C}_{3} \mathrm{C}_{4}$ & $1.426 \mathrm{~A}$ & $\mathrm{~N}_{2} \mathrm{C}_{3} \mathrm{C}_{4}$ & $112.3 \mathrm{~A}$ \\
$\mathrm{C}_{4} \mathrm{C}_{5}$ & $1.357 \mathrm{~A}$ & $\mathrm{C}_{3} \mathrm{C}_{4} \mathrm{C}_{5}$ & $103.0 \mathrm{~A}$ \\
$\mathrm{C}_{5} \mathrm{O}_{2}$ & $1.344 \mathrm{~A}$ & $\mathrm{C}_{4} \mathrm{C}_{5} \mathrm{O}_{1}$ & $110.5 \mathrm{~A}$ \\
$\mathrm{C}_{3} \mathrm{H}$ & $1.078 \mathrm{~B}$ & $\mathrm{C}_{4} \mathrm{C}_{3} \mathrm{H}$ & $129.1 \mathrm{~B}$ \\
$\mathrm{C}_{4} \mathrm{H}$ & $1.074 . \mathrm{B}$ & $\mathrm{C}_{3} \mathrm{C}_{4} \mathrm{H}$ & $128.5 \mathrm{~B}$ \\
$\mathrm{C}_{5} \mathrm{H}$ & $1.074 \mathrm{~B}$ & $\mathrm{C}_{4} \mathrm{C}_{5} \mathrm{H}$ & $133.4 \mathrm{~B}$
\end{tabular}

[1] 0. L. Stiefvater, J. Chem. Phys. 63, 2560 (1975).

[2] O. L. Sticfvater, P. Nosbergor and J. Sheridas, Chem. Phys. 9, 435 (1974). . 


\begin{tabular}{|c|c|c|c|}
\hline \multirow{2}{*}{$\mathrm{C}_{3} \mathrm{H}_{3} \mathrm{NS}$} & \multicolumn{2}{|c|}{ Thiczole } & \\
\hline & & & $C_{8}$ \\
\hline Bond & Substitution & Angle & Substitution \\
\hline $\mathrm{SC}_{2}$ & $1.724 \mathrm{X}$ & $\mathrm{C}_{5} \mathrm{SC}_{2}$ & $89.3 \times$ \\
\hline $\mathrm{C}_{2} \mathrm{~N}$ & 1.304. X & $\mathrm{SC}_{2} \mathrm{~N}$ & $115.1 \mathrm{X}$ \\
\hline $\mathrm{NC}_{4}$ & $1.372 \mathrm{~A}$ & $\mathrm{C}_{2} \mathrm{NC}_{4}$ & $110.1 \times$ \\
\hline $\mathrm{C}_{4} \mathrm{C}_{\bar{z}}$ & $1.367 \times$ & $\mathrm{NC}_{4} \mathrm{C}_{5}$ & $115.8 \mathrm{X}$ \\
\hline $\mathrm{C}_{5} \mathrm{~S}$ & $1.713 \mathrm{X}$ & $\mathrm{C}_{4} \mathrm{C}_{5} \mathrm{~S}$ & $109.5 \mathrm{X}$ \\
\hline $\mathrm{C}_{2} \mathrm{H}_{2}$ & $1.077 \mathrm{X}$ & $5 \mathrm{C}_{2} \mathrm{H}_{2}$ & $121.2 \mathrm{X}$ \\
\hline $\mathrm{C}_{4} \mathrm{H}_{4}$ & $1.080 \mathrm{~A}$ & $\mathrm{NC}_{2} \mathrm{H}_{2}$ & $123.5 \mathrm{X}$ \\
\hline $\mathrm{C}_{5} \mathrm{H}_{5}$ & $1.076 \mathrm{X}$ & $\mathrm{NC}_{4} \mathrm{H}_{4}$ & $119.3 \mathrm{~A}$ \\
\hline & & $\mathrm{C}_{5} \mathrm{C}_{4} \mathrm{H}_{4}$ & $124.8 \times$ \\
\hline & & $\mathrm{C}_{4} \mathrm{C}_{5} \mathrm{H}_{5}$ & $129.0 \times$ \\
\hline & & $\mathrm{SC}_{5} \mathrm{H}_{5}$ & $121.4 \mathrm{X}$ \\
\hline
\end{tabular}

Uncertain structural data caused by a number of small substitution coordinates.

[1] L. Nygaard, E. Asmussen, J. H. Hgig, R. C. Maheshwari, C. H. Nielsen, 1. B. Yetersen, J. Hastrup-Andersen, and 6. $U$. Sørensen, J. Mol. Struct. 8, 225 (1971).

\begin{tabular}{|c|c|c|c|}
\hline \multirow[b]{2}{*}{$\mathrm{C}_{3} \mathrm{H}_{3} \mathrm{~N}_{3}$} & \multicolumn{2}{|c|}{ s-Triazine } & \multirow[b]{2}{*}{$\mathrm{D}_{3}$} \\
\hline & \multicolumn{2}{|c|}{$\mathrm{N}=\mathrm{CH}-\mathrm{N}=\mathrm{CH}-\mathrm{N}=\mathrm{CH}$} & \\
\hline Bond & Effective & Angle & Effective \\
\hline $\mathrm{CN}$ & $\mathrm{I} .338 \mathrm{X}$ & NCN & $127 \mathrm{X}$ \\
\hline & & & \\
\hline
\end{tabular}

In order to obtain the structural parameters it was necessary to assume the $\mathrm{CH}$ bond distances. Since the uncertainties in the other parameters are dependent upon this assumption, their uncertainties are given an $\mathrm{X}$ rating.

[1] J. E. Lancaster and B. P. Stoicheff, Can. J. Phys. 34, 1016 (1956).

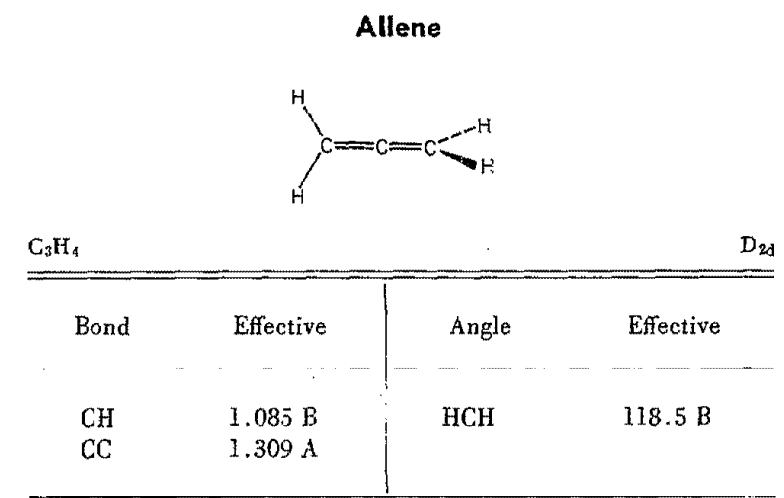

[1] A. G. Maki and R. A. Toth, J. Mol. Spectrosc. 17, 136 (1965).

[2] R. J. Butcher and W. J. Jones, J. Raman Spectrosc. I, 393 (1973).

\section{Cyclopropene}

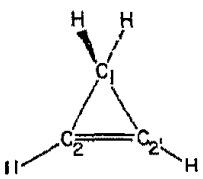

$\mathrm{C}_{3} \mathrm{H}_{4}$

\begin{tabular}{ll|ll}
\hline \multicolumn{2}{c}{$\mathrm{C}_{3} \mathrm{H}_{4}$} & & $\mathrm{C}_{2 \mathrm{r}}$ \\
\hline Bond & Substitution & Angle & Substitution \\
\hline & & & \\
$\mathrm{C}_{1} \mathrm{C}_{2}$ & $1.509 \mathrm{~B}$ & $\mathrm{C}_{1} \mathrm{C}_{2} \mathrm{C}_{2^{\prime}}$ & $50.8 \mathrm{~A}$ \\
$\mathrm{C}_{2} \mathrm{C}_{2^{\prime}}$ & $1.296 \mathrm{~A}$ & $\mathrm{C}_{2} \mathrm{C}_{2^{\prime}} \mathrm{H}$ & $149.9 \mathrm{~B}$ \\
$\mathrm{C}_{2} \mathrm{H}$ & $1.072 \mathrm{~B}$ & $\mathrm{HCH}$ & $114.6 \mathrm{~B}$ \\
$\mathrm{C}_{1} \mathrm{H}$ & $1.088 \mathrm{~B}$ & & \\
\hline
\end{tabular}

[1] W. M. Stigliani, V. W. Laurie and J. C. Li, J. Chem. Phys. 62, 1890 (1975).

[2] P. H. Kasai, R. J. Myers, D. F. Eggers and K. B. Wiberg, J. Chem. Phys. 30, $512(1959)$.

\section{Methyl acetylene}

\begin{tabular}{ll|ll}
$\mathrm{C}_{3} \mathrm{H}_{4}$ & $\mathrm{H}_{3} \mathrm{C}^{3}-\mathrm{C}^{2} \equiv \mathrm{C}^{3}-\mathrm{H}$ & $\mathrm{C}_{3 \mathrm{r}}$ \\
\hline Bond & Substitution & Angle & Substitution \\
\hline & & & \\
$\mathrm{C}^{1} \mathrm{C}^{2}$ & $1.459 \mathrm{~B}$ & $\mathrm{HC}^{1} \mathrm{C}^{2}$ & $110.2 \mathrm{~B}$ \\
$\mathrm{C}^{2} \mathrm{C}^{3}$ & $1.206 \mathrm{~B}$ & & \\
$\mathrm{C}^{3} \mathrm{H}$ & $1.056 \mathrm{~B}$ & & \\
$\mathrm{C}^{1} \mathrm{H}$ & $1.105 \mathrm{~B}$ & &
\end{tabular}

[1] C. C. Costain, J. Chem. Phys. 29, 864 (1958).

[2] R. Trambarulo and W. Gordy, J. Chem. Phys. 18, 1613 (1950).

[3] L. F. Thomlas, E. I. Sherrard and J. Skeridan, Trans. Faraday Soc. 51,619 (1955). 


\section{1,1-Dichlorocyclopropane}

\begin{tabular}{|c|c|c|c|}
\hline $\mathrm{C}_{3} \mathrm{H}_{4} \mathrm{Cl}_{2}$ & \multicolumn{2}{|c|}{$\mathrm{Cl}_{2} \mathrm{C}^{1}-\mathrm{C}^{2} \mathrm{H}_{2}-\mathrm{C}^{3} \mathrm{H}_{2}$} & $\mathrm{C}_{24}$ \\
\hline Bond & Substitution & Angle & Substitution \\
\hline $\mathrm{CH}$ & $1.085 \mathrm{~B}$ & $\mathrm{HCH}$ & $117.5 \mathrm{~B}$ \\
\hline $\mathrm{C}^{2} \mathrm{C}^{3}$ & $1.534 \mathrm{~B}$ & $\mathrm{C}^{2} \mathrm{C}^{3} \mathrm{H}$ & $117.5 \mathrm{~B}$ \\
\hline $\mathrm{C}^{1} \mathrm{C}^{2}$ & $1.532 \mathrm{C}$ & $\mathrm{ClCCl}$ & $114.2 \mathrm{C}$ \\
\hline $\mathrm{CCl}$ & $1.734 \mathrm{C}$ & $\mathrm{C}^{2} \mathrm{C}^{\mathrm{x}} \mathrm{C}^{3}$ & $60.1 \mathrm{~B}$ \\
\hline
\end{tabular}

[1] W. H. Flygare, A. Narath, and W. D. Gwinn, J. Chem. Phys. 36, $200(1962)$.

\section{1,1-Difluorocyclopropane}

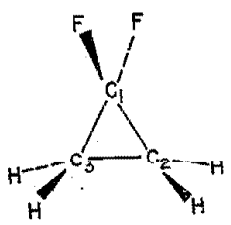

$\mathrm{C}_{2} \mathrm{H}_{4} \mathrm{~F}_{2}$

\begin{tabular}{ll|ll}
\hline Bond & Substitution & Angle & Substitution \\
\hline $\mathrm{C}_{1} \mathrm{C}_{2}$ & $1.464 \mathrm{C}$ & $\mathrm{FCH}$ & $108.3 \mathrm{C}$ \\
$\mathrm{C}_{2} \mathrm{C}_{3}$ & $1.553 \mathrm{~A}$ & $\mathrm{HCH}$ & $116.9 \mathrm{~B}$ \\
$\mathrm{CF}$ & $1.355 \mathrm{C}$ & & \\
$\mathrm{CH}$ & $1.082 \mathrm{~B}$ & & \\
\hline
\end{tabular}

Structural parameters were evaluated for the $\mathrm{D}_{4}$ species.

[1] A. T. Perretta and V. W. Laurie, J. Chem. Phys. 62, 2469 (1975).

\section{1,3-Dinuorocacetone}

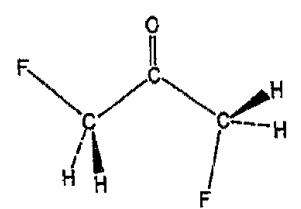

$\mathrm{C}_{3} \mathrm{H}_{4} \mathrm{~F}_{2} \mathrm{O}$

$\mathrm{C}_{\mathrm{s}}$

The observed spectra were consistent with the above conformation. Intensity measurements suggest that this species may represent only about $20 \%$ of the gas-phase sample.

[1] D. J. Finnigan, C. W. Cilliee, R. D. Suenram and E. B. Wileon, J. Mol. Spectrosc. 57, 363 (1975).
Pyrazole

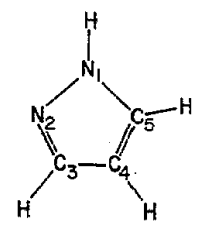

$\mathrm{C}_{3} \mathrm{H}_{4} \mathrm{~N}_{2}$

$\mathrm{C}_{\text {m }}$

\begin{tabular}{ll|ll}
\hline \hline Bond & Substitution & Angle & Substitution \\
\cline { 3 - 4 } & & & \\
\cline { 3 - 4 } $\mathrm{N}_{1} \mathrm{~N}_{2}$ & $1.349 \mathrm{~B}$ & $\mathrm{~N}_{1} \mathrm{~N}_{2} \mathrm{C}_{3}$ & $104.1 \mathrm{~B}$ \\
$\mathrm{~N}_{2} \mathrm{C}_{3}$ & $1.331 \mathrm{~B}$ & $\mathrm{~N}_{2} \mathrm{C}_{3} \mathrm{C}_{4}$ & $111.9 \mathrm{~B}$ \\
$\mathrm{C}_{3} \mathrm{C}_{4}$ & $1.416 \mathrm{~B}$ & $\mathrm{C}_{3} \mathrm{C}_{4} \mathrm{C}_{5}$ & $104.5 \mathrm{~B}$ \\
$\mathrm{C}_{4} \mathrm{C}_{3}$ & $1.372 \mathrm{~A}$ & $\mathrm{C}_{4} \mathrm{C}_{5} \mathrm{~N}_{1}$ & $106.4 \mathrm{~B}$ \\
$\mathrm{C}_{5} \mathrm{~N}_{1}$ & $1.359 \mathrm{~B}$ & $\mathrm{C}_{5} \mathrm{~N}_{1} \mathrm{~N}_{2}$ & $113.1 \mathrm{~B}$ \\
$\mathrm{~N}_{2} \mathrm{H}$ & $0.998 \mathrm{~B}$ & $\mathrm{~N}_{2} \mathrm{C}_{3} \mathrm{H}$ & $119.3 \mathrm{~B}$ \\
$\mathrm{C}_{3} \mathrm{H}$ & $1.078 \mathrm{~B}$ & $\mathrm{C}_{3} \mathrm{C}_{4} \mathrm{H}$ & $127.9 \mathrm{~B}$ \\
$\mathrm{C}_{4} \mathrm{H}$ & $1.076 \mathrm{~B}$ & $\mathrm{~V}_{1} \mathrm{C}_{5} \mathrm{H}$ & $121.5 \mathrm{~B}$ \\
$\mathrm{C}_{5} \mathrm{H}$ & $1.077 \mathrm{~B}$ & $\mathrm{~N}_{2} \mathrm{~N}_{1} \mathrm{H}$ & $118.4 \mathrm{~B}$ \\
& & & \\
\hline
\end{tabular}

[1] L. Nygaard, D. Christen, J. T. Nielsen, E. J. Pedersen, 0. Snerling, E. Vestergaard and G. O. Sorensen, J. Mol. Struct. 22, 401 (1974).

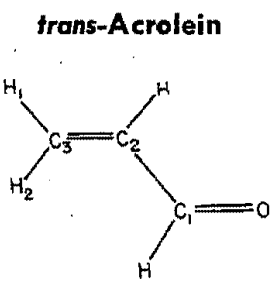

$\mathrm{C}_{3} \mathrm{H}_{4} \mathrm{O}$

$\mathrm{C}_{\mathrm{B}}$

\begin{tabular}{lll|lll}
\hline \hline Bond & Substitution & Effective & Angle & Substitution & Effective \\
\hline & & & & & \\
$\mathrm{CO}$ & $1.219 \mathrm{C}$ & & $\mathrm{CCO}$ & $123.3 \mathrm{C}$ & \\
$\mathrm{C}_{1} \mathrm{C}_{2}$ & $1.470 \mathrm{~B}$ & & $\mathrm{C}_{2} \mathrm{C}_{1} \mathrm{H}$ & $115 . \mathrm{B}$ & \\
$\mathrm{C}_{1} \mathrm{H}$ & $1.108 \mathrm{~B}$ & & $\mathrm{C}_{1} \mathrm{C}_{2} \mathrm{H}$ & $117.3 \mathrm{~B}$ & \\
$\mathrm{C}_{2} \mathrm{H}$ & $1.084 \mathrm{~B}$ & & $\mathrm{C}_{1} \mathrm{C}_{2} \mathrm{C}_{8}$ & & $119.8 \mathrm{C}$ \\
$\mathrm{C}_{2} \mathrm{C}_{3}$ & & $1.345 \mathrm{~B}$ & $\mathrm{C}_{2} \mathrm{C}_{3} \mathrm{H}_{1}$ & & $121.5 \mathrm{C}$ \\
$\mathrm{C}_{3} \mathrm{H}_{1}$ & & $1.086 \mathrm{C}$ & $\mathrm{C}_{2} \mathrm{C}_{3} \mathrm{H}_{2}$ & & $120.0 \mathrm{C}$ \\
$\mathrm{C}_{3} \mathrm{H}_{2}$ & & $1.086 \mathrm{C}$ & & & \\
& & & & & \\
\hline
\end{tabular}

The $\mathrm{C}_{3} \mathrm{H}_{1}$ and $\mathrm{C}_{3} \mathrm{H}_{2}$ distances have been assumed to be equal. [1] E. A. Cherniak and C. C. Costain, J. Chem. Phys. 45, 104 (1966). 


\section{Cyclopropanone}

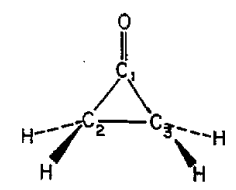

$\mathrm{C}_{3} \mathrm{H}_{4} \mathrm{O}$

\begin{tabular}{ll|lc}
\hline \hline Bond & Substitution & Angle & Substitution \\
\hline & & & \\
$\mathrm{CO}$ & $1.191 \mathrm{E}$ & $\mathrm{C}_{1} \mathrm{C}_{2} \mathrm{C}_{3}$ & $57.7 \mathrm{C}$ \\
$\mathrm{C}_{2} \mathrm{C}_{2}$ & $1.475 \mathrm{D}$ & $\mathrm{C}_{2} \mathrm{C}_{2} \mathrm{C}_{3}$ & $64.6 \mathrm{C}$ \\
$\mathrm{C}_{2} \mathrm{C}_{3}$ & $1.575 \mathrm{D}$ & $\mathrm{HCH}$ & $114.1 \mathrm{D}$ \\
$\mathrm{CH}$ & $1.086 \mathrm{D}$ & $\mathrm{HC}_{2} \mathrm{C}_{1}$ & $118.5 \mathrm{D}$ \\
& & $\mathrm{HC}_{2} \mathrm{C}_{3}$ & $118.3 \mathrm{D}$ \\
& & & \\
\hline
\end{tabular}

[I] J. M. Pochan, J. E. Baldwin and W. H. Flygare, J. Amer. Chem. Soc. 91, 1896 (1969).

[2] J. M. Poohan, J. E. Baldwin and W. H. Flygare, J. Amer. Chem. Soc. 90, 1072 (1968).

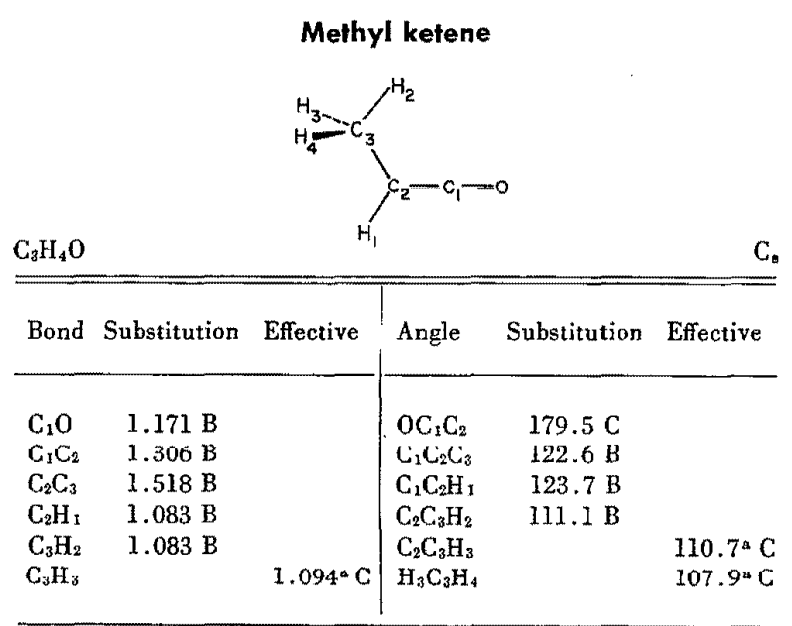

"Recalculated from the original data.

[1] B. Bak, J. J. Christiansen, K. Kunstmann, L. Nygaard and J. Rastrup-Andersen, J. Chem. Phys. 45, 883 (1966).

\section{Malonaldehyde}

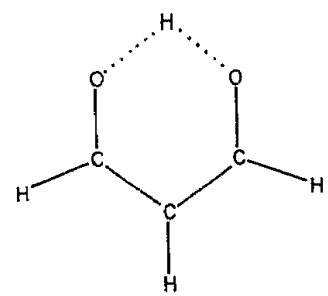

$\mathrm{C}_{3} \mathrm{II}_{4} \mathrm{O}_{2}$

$\mathrm{C}_{2 v}$

Experimental evidence supports a structure with average $\mathrm{C}_{2 v}$ sym. metry. A low-barrier double-minimum hydrogen-bond potential function may exist. The $0 \cdots 0$ distanec was found to be $2.55(\mathrm{X})$.

[1] W. F. Rowe, Jr., R. W. Duerst, and E. B. Wilson, J. Amer. Chem. Soc. 98, 4021 (1976).

\section{3-Oxetanone}

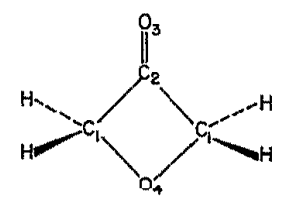

$\mathrm{C}_{3} \mathrm{H}_{4} \mathrm{O}_{2}$

\begin{tabular}{|c|c|c|c|c|c|}
\hline Bond & Substitution & Effective & Angle & Substitution & Effective \\
\hline $\mathrm{C}_{2} \mathrm{C}_{2}$ & $1.522 \mathrm{~B}$ & & $\mathrm{C}_{1} \mathrm{C}_{2} \mathrm{C}_{1}$ & $88.1 \mathrm{~B}$ & \\
\hline $\mathrm{C}_{1} \mathrm{O}_{4}^{\mathrm{ta}}$ & & $1.450 \mathrm{X}$ & $\mathrm{C}_{2} \mathrm{C}_{1} \mathrm{O}_{4}^{\mathrm{B}}$ & & $88.5 \mathrm{X}$ \\
\hline $\mathrm{C}_{2} \mathrm{O}_{3^{\mathrm{a}}}^{\mathrm{n}}$ & & $1.230 \mathrm{X}$ & $\mathrm{C}_{1} \mathrm{O}_{4} \mathrm{C}_{1}{ }^{8}$ & & $94.8 \mathrm{X}$ \\
\hline
\end{tabular}

a In order to obtain parametere invalving oxygen atoms, the methylene group parameters were assumed.

[1] J. S. Gibson and D. O. Harris, J. Chem. Phys. 57, 2318 (1972).

\section{Oxiranecarboxaldehyde}

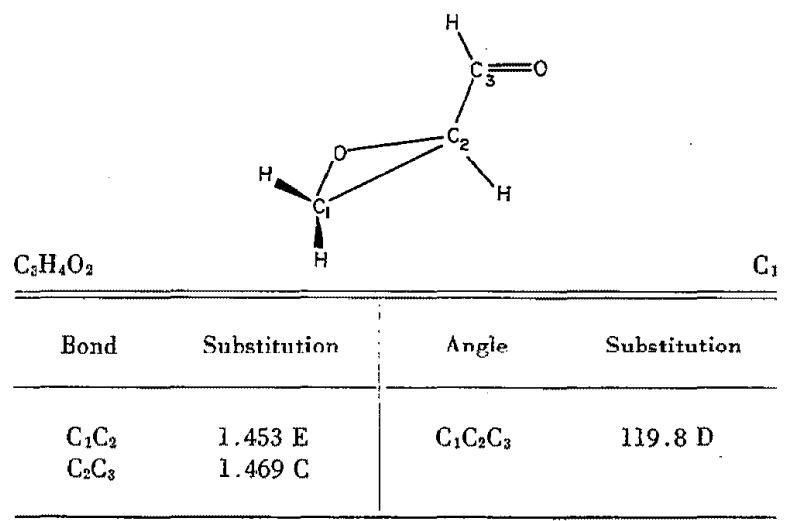

[1] R. A. Creswell, P. J. Manor, R. A. Assink and R. H. Schwendeman, J. Mol. Spectrosc. 64, 365 (1977). 
Ethynyl Methyl Sulfide

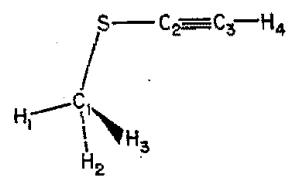

$\mathrm{C}_{3} \mathrm{H}_{4} \mathrm{~S}$

\begin{tabular}{lll|lll}
\hline \hline Bond Substitution & Effective & Angle & Substitution & Effective \\
\hline $\mathrm{SC}_{1}$ & $1.813 \mathrm{~B}$ & & $\mathrm{C}_{1} \mathrm{SC}_{2}$ & & \\
$\mathrm{SC}_{2}$ & & $1.685 \mathrm{C}$ & $\mathrm{H}_{1} \mathrm{C}_{1} \mathrm{H}_{2}$ & $110.0 \mathrm{~B}$ & $99.9 \mathrm{C}$ \\
$\mathrm{C}_{2} \mathrm{C}_{3}$ & & $1.205 \mathrm{C}$ & $\mathrm{SC}_{2} \mathrm{C}_{3}$ & & $178.0 \mathrm{C}$ \\
$\mathrm{C}_{3} \mathrm{H}_{4}$ & & $1.061 \mathrm{C}$ & $\mathrm{H}_{2} \mathrm{C}_{1} \mathrm{H}_{3}$ & $110.3 \mathrm{~B}$ & \\
$\mathrm{C}_{1} \mathrm{H}_{1}$ & $1.079 \mathrm{~B}$ & & & & \\
$\mathrm{C}_{1} \mathrm{H}_{2}$ & $1.090 \mathrm{~B}$ & & & & \\
\hline
\end{tabular}

The $\mathrm{C}_{2} \mathrm{C}_{3} \mathrm{H}_{4}$ angle was assumed to be $180^{\circ}$. The acetylene group is tilted away from the methyi group. The methyl group is tilted apm proximately $2^{\circ}$ away from the acetylene group.

[1] D. den Engelsen, J. Mol. Spectrose. 30, 474 (1969).

[2] D. den Engelsen, J. Mol. Spectrosc. 22, 426 (1967).

\section{Chlorocyclopropane}

(Cyclopropyl chloride)

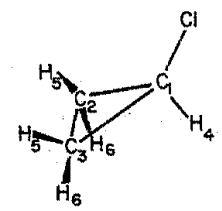

$\mathrm{C}_{3} \mathrm{H}_{5} \mathrm{Cl}$

\begin{tabular}{ll|ll}
\hline \hline Bond & Substitution & Angle & Substitution \\
\hline & & & \\
$\mathrm{CCl}$ & $1.7410 \mathrm{C}$ & $\mathrm{CCCl}$ & $118.7 \mathrm{C}$ \\
$\mathrm{C}_{1} \mathrm{C}_{2}$ & $1.513 \mathrm{C}$ & $\mathrm{ClCH}$ & $115.8 \mathrm{C}$ \\
$\mathrm{C}_{2} \mathrm{C}_{3}$ & $1.515 \mathrm{~B}$ & $\mathrm{CCH}_{4}$ & $116.1 \mathrm{C}$ \\
$\mathrm{CH}_{4}$ & $1.079 \mathrm{~B}$ & $\mathrm{C}_{1} \mathrm{C}_{3} \mathrm{H}_{5}$ & $115.5 \mathrm{C}$ \\
$\mathrm{CH}_{5}$ & $1.086 \mathrm{C}$ & $\mathrm{C}_{1} \mathrm{C}_{3} \mathrm{H}_{6}$ & $117.8 \mathrm{C}$ \\
$\mathrm{CH}_{6}$ & $1.082 \mathrm{C}$ & $\mathrm{C}_{2} \mathrm{C}_{3} \mathrm{H}_{5}$ & $116.9 \mathrm{C}$ \\
& & $\mathrm{C}_{2} \mathrm{C}_{3} \mathrm{H}_{6}$ & $118.7 \mathrm{C}$ \\
& & $\mathrm{HCH}$ & $116.2 \mathrm{C}$ \\
& & & \\
\hline $\mathrm{C}$
\end{tabular}

[1] R. H. Schwendeman, G. D. Jacobs and T. M. Krigas, J. Chem. Phys. 40, 1022 (1964).
cis-1-Chloropropene

$\mathrm{C}_{3} \mathrm{H}_{5} \mathrm{Cl}$

$\mathrm{CH}_{3}-\mathrm{CH}=\mathrm{CHCl}$

$\mathrm{C}_{\mathrm{B}}$

Assuming all other parameters to be the same as in propene, $\mathrm{C}-\mathrm{Cl}=1.735(\mathrm{X})$ and $\mathrm{C}=\mathrm{C}-\mathrm{Cl}=125.2(\mathrm{X})$. A trans form was also investigated and similar assumptions led to $\mathrm{C}-\mathrm{Cl}=1.728(\mathrm{X})$ and $\mathrm{C}=\mathrm{C}-\mathrm{Cl}=\mathrm{I} 21.9(\mathrm{X})$.

[1] R. A. Beaudet, J. Chem. Phys. 40, 2705 (1964).

[2] R. A. Beaudet, J. Chem. Phys. 37, 2398 (1962).

\section{2-Chloropropene}

$\mathrm{C}_{3} \mathrm{H}_{5} \mathrm{Cl}$

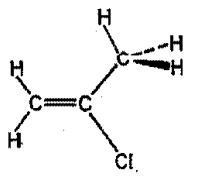

$\mathrm{C}_{\mathrm{s}}$

The equilibrium conformation of the methyl group was found to be that given in figure. The authors have also given a reasonable structure based on assumed parameters from propene and vinyl chloride.

[1] W. Good, R. J. Conan, A. Bauder and Hs. H. Gunthard, J. Mol. Spectrosc. 41, 381 (1972).

$\lceil 2\rceil$ M. L. Unland, V. Weiss and W. H. Flygare, J. Chem. Phys. 42, $2138(1965)$

cis-3-Chloropropene

$\mathrm{C}_{6} \mathrm{H}_{5} \mathrm{Cl}$

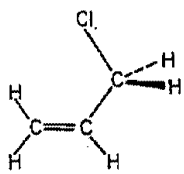

$\mathrm{C}_{n}$

Rotational constants for two chlorine isotopic species were in agrecrnent with a plane of symmetry. $A$ socond rotamcr, with a dihedral angle of $122^{\circ}$ about $\mathrm{C}-\mathrm{C}$ bond, was also observed.

[1] E. Hirota, J. Mol. Spectrosc. 35, 9 (1970).

\section{Propanoyl Chloride \\ (Propionyl Chloride)}

$\mathrm{C}_{3} \mathrm{H}_{5} \mathrm{ClO}$

$\mathrm{CH}_{3} \mathrm{CH}_{2} \mathrm{COCl}$

$\mathrm{C}_{\mathrm{s}}$

The most stable isomer was determined as a heavy atom planar configuration with the methyl group and the carbonyl oxygen atom cis to each other.

[1] H. Karlsson, J. Mol. Struct. 33, 227 (1976). 
cis-3-Flvoropropene<smiles>C=C(C)C(C)(C)F</smiles>

$\mathrm{C}_{3} \mathrm{H}_{5} \mathrm{~F}$

\begin{tabular}{ll|ll}
\hline Bnno & Suhstitution & Angle & Substitution \\
\hline & & & \\
\hline$C_{1} \mathrm{C}_{2}$ & $1.333 \mathrm{C}$ & $\mathrm{C}_{1} \mathrm{C}_{2} \mathrm{C}_{3}$ & $124.6 \mathrm{C}$ \\
$\mathrm{C}_{2} \mathrm{C}_{3}$ & $1.488 \mathrm{C}$ & $\mathrm{C}_{2} \mathrm{C}_{3} \mathrm{~F}$ & $111.7 \mathrm{C}$ \\
$\mathrm{C}_{2} \mathrm{~F}$ & $1.382 \mathrm{C}$ & $\mathrm{C}_{2} \mathrm{C}_{1} \mathrm{H}_{1}$ & $120.9 \mathrm{C}$ \\
$\mathrm{C}_{3} \mathrm{H}_{1}$ & $1.080 \mathrm{C}$ & $\mathrm{C}_{2} \mathrm{C}_{1} \mathrm{H}_{2}$ & $119.2 \mathrm{C}$ \\
$\mathrm{C}_{1} \mathrm{H}_{2}$ & $1.105 \mathrm{C}$ & $\mathrm{H}_{2} \mathrm{C}_{2} \mathrm{H}_{2}$ & $119.9 \mathrm{C}$ \\
$\mathrm{C}_{3} \mathrm{H}_{4}$ & $1.098 \mathrm{C}$ & $\mathrm{C}_{2} \mathrm{C}_{3} \mathrm{H}_{4}$ & $111.1 \mathrm{C}$ \\
$\mathrm{C}_{3} \mathrm{H}_{5}$ & $1.098 \mathrm{C}$ & $\mathrm{C}_{2} \mathrm{C}_{3} \mathrm{H}_{3}$ & $111.1 \mathrm{C}$ \\
& & $\mathrm{H}_{4} \mathrm{C}_{3} \mathrm{H}_{3}$ & $108.1 \mathrm{C}$ \\
& & $\mathrm{H}_{4} \mathrm{C}_{3} \mathrm{~F}$ & $107.4 \mathrm{C}$ \\
& & $\mathrm{H}_{3} \mathrm{C}_{3} \mathrm{~F}$ & $107.4 \mathrm{C}$ \\
\hline
\end{tabular}

$\mathrm{C}_{2} \mathrm{H}_{3}$ and $\mathrm{C}_{1} \mathrm{C}_{2} \mathrm{H}_{3}$ parameters assumed.

[1] E. Hirota, J. Chem. Phys. 42, 2071 (1965).

gauche-3-Fluoropropene

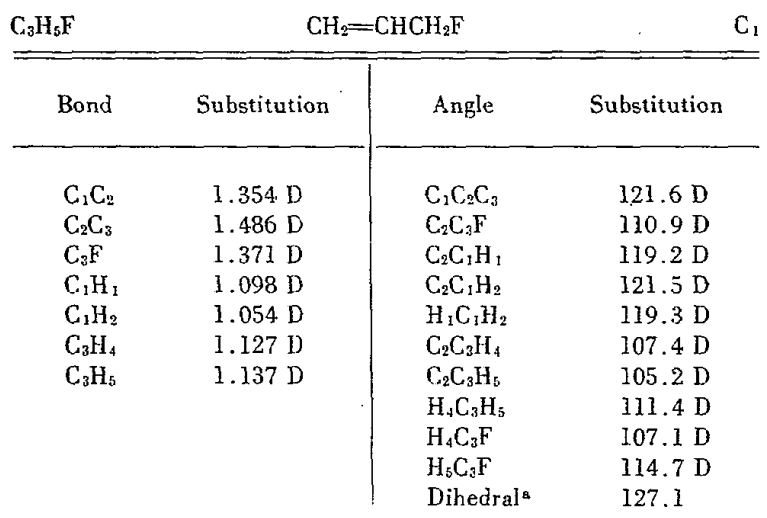

Gauche form has $\mathrm{CH}_{2} \mathrm{~F}$ rotated 127.1 relative to cis. See cis-3 fluoropropene structure. $\mathrm{C}_{2} \mathrm{H}_{3}$ and $\mathrm{C}_{1} \mathrm{C}_{2} \mathrm{H}_{3}$ parameters assumed. [1] E. Hirota, J. Chem. Phys. 42, 2071 (1965).

\section{1-Fluoro-2-propanone (Fluorocacetone)}

$\mathrm{C}_{3} \mathrm{H}_{5} \mathrm{FO}$

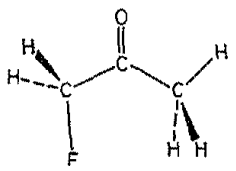

$\mathrm{C}_{s}$

No quantitative structural information other than the conformation of the most stable rotamer was obtained.

[1] E. Saegerbarth and L. Krisher, J. Chem. Phys. 52, 3555 (1970).

\section{cis-Propionyl Fluoride}

$\mathrm{C}_{3} \mathrm{H}_{5} \mathrm{FO}$

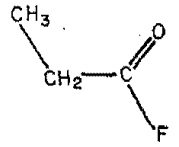

$\mathrm{C}_{\mathrm{s}}$

Other than establishing this confornation as the most stable, no quantitative information was obtained about the structure.

A gauche form, $1290 \mathrm{cal} / \mathrm{mol}$ less stable, was also observed. See the following.

[1] O.Stiefvater and E. B. Wilson, J. Chem. Phys. 50, 5385 (1969).

\section{gauche-Propionyl Fluoride}

$\mathrm{C}_{0} \mathrm{H}_{6} \mathrm{FO}$

$\mathrm{CH}_{2} \mathrm{CH}_{2} \mathrm{COF}$

$C_{1}$

Dihedral angle between CCC and COF plane is $120^{\circ}$ (E). A cis form, $1290 \mathrm{cal} / \mathrm{mol}$ more stable, was also observed. See the preceding.

[1] O. Stiefvater and E. B. Wilson, J. Chem. Phys. 50, 5385 (1969).

\section{Propanenitrile \\ (Ethyl cyanide)}

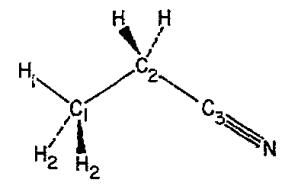

$\mathrm{C}_{3} \mathrm{H}_{5} \mathrm{~N}$

\begin{tabular}{ll|ll}
\hline \hline Bond & Substitution & Angle & Substitution \\
\hline & & & \\
$\mathrm{C}_{1} \mathrm{C}_{2}$ & $1.537 \mathrm{~B}$ & $\mathrm{C}_{2} \mathrm{C}_{3} \mathrm{~N}$ & $178.7^{\mathrm{a}} \mathrm{D}$ \\
$\mathrm{C}_{2} \mathrm{C}_{3}$ & $1.459 \mathrm{C}$ & $\mathrm{C}_{1} \mathrm{C}_{2} \mathrm{C}_{3}$ & $112.0 \mathrm{~B}$ \\
$\mathrm{CN}$ & $1.159 \mathrm{C}$ & $\mathrm{C}_{2} \mathrm{C}_{2} \mathrm{H}$ & $110.6 \mathrm{~B}$ \\
$\mathrm{C}_{2} \mathrm{H}$ & $1.094 \mathrm{~B}$ & $\mathrm{HC}_{2} \mathrm{C}_{3}$ & $108 . \mathrm{I} \mathrm{B}$ \\
$\mathrm{C}_{2} \mathrm{H}_{1}$ & $1.079 \mathrm{D}$ & $\mathrm{HC}_{2} \mathrm{H}$ & $107.2 \mathrm{~B}$ \\
$\mathrm{C}_{1} \mathrm{H}_{2}$ & $1.091 \mathrm{~B}$ & $\mathrm{H}_{2} \mathrm{C}_{1} \mathrm{H}_{2}$ & $107.8 \mathrm{~B}$ \\
& & $\mathrm{II}_{2} \mathrm{C}_{1} \mathrm{C}_{2}$ & $110.5 \mathrm{~B}$ \\
& & $\mathrm{H}_{1} \mathrm{C}_{1} \mathrm{C}_{2}$ & $110.1 \mathrm{E}$ \\
\hline
\end{tabular}

a The CN group is bent slightly toward the methyl group.

[1] H. M. Heise, H. Lutz and H. Dreizler, Z. Naturforsch. 29a, 3345 (1974). 


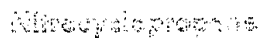

$\mathrm{C}_{3} \mathrm{H}_{6} \mathrm{NO}_{2}$
Dens not

$S_{S}$

C.

$\left(\mathrm{CH}_{3}\right) \mathrm{MON}$

$\mathrm{C}_{5}$
The conformation of the molecule is iound to be the bisected one with the plane of the nitro yroup perpen aicular to the piane of the cyclopropyl ring.

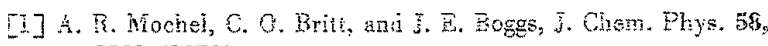
$322 i(1973)$.

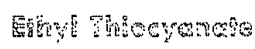

$\mathrm{C}_{3} \mathrm{i}, \mathrm{NS}$

$\mathrm{CH}_{3} \mathrm{CESCH}$

C:

One rotatona isomer, having the CHs gron and sCr groun gauche to one arother, was ilantified.

[1] A. Bjprseth and K. M. Marstohi, J. Mol. Stuct. 21. 15 (1972),

\begin{tabular}{|c|c|c|c|}
\hline \multicolumn{4}{|c|}{ Gych arome } \\
\hline$c_{3} \underline{\underline{A}}_{5}$ & \multicolumn{2}{|c|}{$\mathrm{CH}$} & $D_{n}$ \\
\hline Bond & Encclive & Anglo & Efiective \\
\hline $\begin{array}{ll}\mathrm{CI} \\
\mathrm{CO}\end{array}$ & $\begin{array}{l}3.088 \mathrm{C} \\
1.5 \mathrm{i} 2 \mathrm{C}\end{array}$ & $\mathrm{HCL}$ & 31.00 \\
\hline
\end{tabular}

In the calewlation, $\mathrm{o}(\mathrm{C}-\mathrm{H})-\mathrm{n}(\mathrm{C}-\mathrm{D})=0.002$ i was assumed.

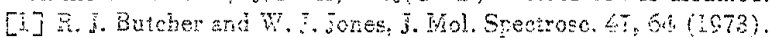

[2] J. J. Jones ent B. P. Stoicheff, Can. J. Phys, 42, 2259 (1964).

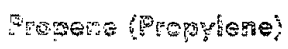

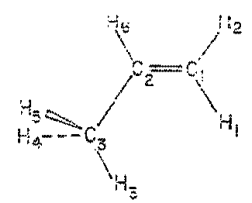

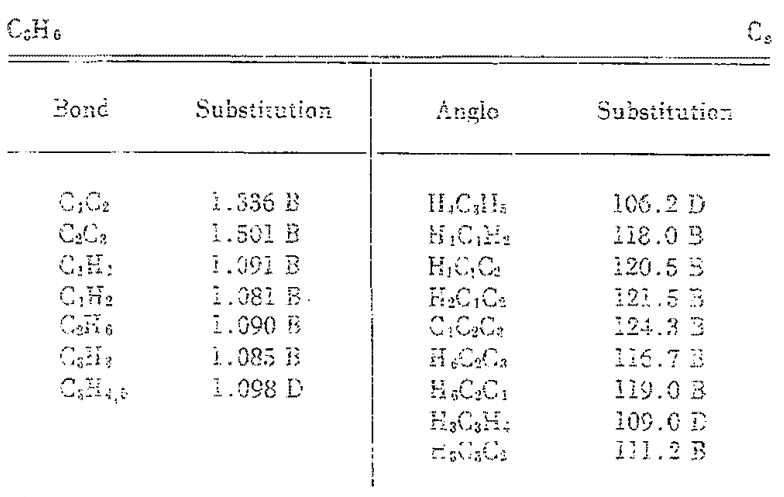

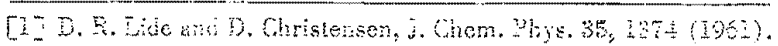

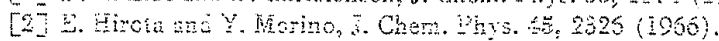

It was ollown that the heavy atom skieton is non-bianar.

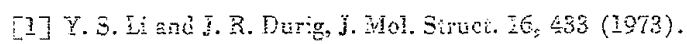

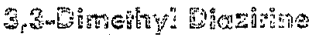

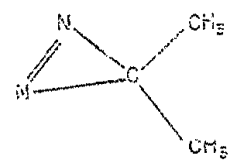

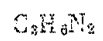

\begin{tabular}{|c|c|c|c|}
\hline Bond & Effective & Anggle & Effective \\
\hline $\mathrm{CC}$ & $1.50 \mathrm{X}$ & $\mathrm{CC}$ & $120 x$ \\
\hline $\mathrm{CN}$ & $1.40 \mathrm{I}$ & $\mathrm{NA}_{3} \mathrm{CH}_{3}$ & $100 x$ \\
\hline DNT & $1.235 \mathrm{P}$ & 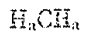 & $108 x$ \\
\hline $\mathrm{CH}_{\mathrm{s}}$ & $\$ .08 \%$ & & \\
\hline$C W_{\mathrm{n}}$ & $1.10 X$ & & \\
\hline
\end{tabular}

TVCN and NNC angies assumed. $H_{5}$ and $\mathrm{H}_{n}$ reier co hydrogen atons in and out of the plane of symmetry, respectively.

[I] J. E. Wolirab, L. K. Sharpen, D. Y. Ames, and I. A. Vererit, J. Chem. Why. 49,2208 (1965).

\begin{tabular}{|c|c|c|c|}
\hline \multicolumn{4}{|c|}{ A getore } \\
\hline $\mathrm{C}_{3} \mathrm{H}_{6} \mathrm{O}$ & \multicolumn{2}{|c|}{$\mathrm{CF}_{3} \mathrm{COCH}_{3}$} & $\mathrm{C}_{27}$ \\
\hline Bond & Subetitution & Argle & Substitedion \\
\hline $\mathrm{CO}$ & $1.507 \mathrm{~B}$ & $\cos$ & $117.2 \mathrm{I3}$ \\
\hline $\mathrm{CO}$ & $1.222 \pi$ & $\mathrm{HCI}$ & $1033.7 \mathrm{~g}$ \\
\hline $\mathrm{CH}$ & $\vdots .085 \mathrm{C}$ & 20 & $119.9 \mathrm{~B}$ \\
\hline
\end{tabular}

Niethyl group assumed to be symmetrical. Internal rotation anplysis movided 20 , the angle between symeiny axes of methy groups.

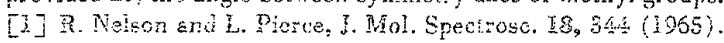

22 J. B. Swalen and G. E. Coswin, J. Chem. Whys. 31 , is62 (igso).

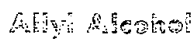

$\mathrm{C}_{3} \mathrm{HO}_{0}$

$\mathrm{CH}=\mathrm{CHCHOH}$

$\mathrm{G}_{\tilde{z}}$

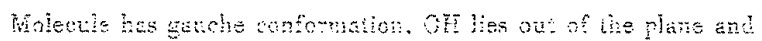

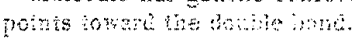

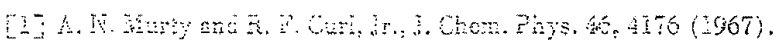




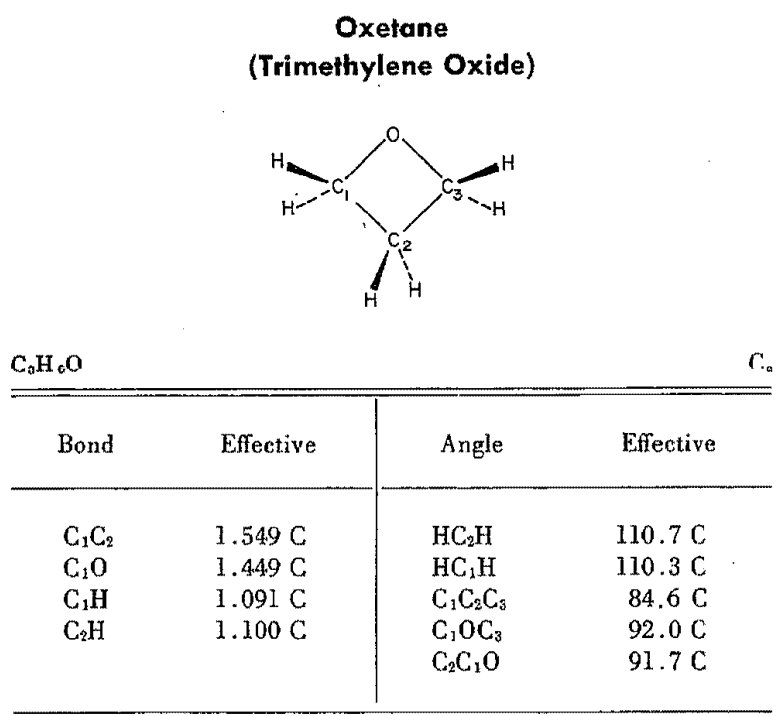

Because of low barrier, the effective symmetry is $\mathrm{C}_{2 \gamma}$.

[1] S. I. Chan. J. Zinn and W. D. Gwinn, J. Chem. Phys. 34, 1319 (1961).

\section{cis-Propionaldehyde}

$\mathrm{C}_{3} \mathrm{H}_{6} \mathrm{O}$

$\mathrm{CH}_{3} \mathrm{CH}_{2} \mathrm{CHO}$

$\mathrm{C}_{\mathrm{s}}$

Gauche form also observed. Carbonyl eclipses methyl group but no detailed structure available.

[1] S. S. Butcher and E. B. Wilson, J. Chem. Phys. 40, 1671 (1964).

gouche-Propionaldehyde

\begin{tabular}{ccc} 
& $\mathrm{CH}_{3} \mathrm{CH}_{2} \mathrm{CHO}$ & $\mathrm{C}_{3} \mathrm{H}_{6} \mathrm{O}$ \\
\hline Angle & Effective \\
\hline$\theta^{\mathrm{a}}$ & $131 \mathrm{X}$
\end{tabular}

a $\theta$ is angle of rotation of carbonyl group from cis conformation where carbonyl eclipses methyl group.

[1] S. S. Butcher and E. B. Wilson, J. Chem. Phys. 40, 1671 (1964).

\section{Methoxyacetic Acid}

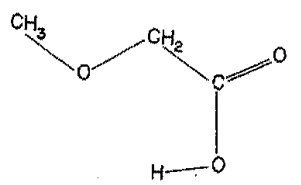

$\mathrm{C}_{3} \mathrm{H}_{6} \mathrm{O}_{3}$

Symmetry plane and conformation seem well established, but no detailed structure is available.

[1] K. M. Marstokk and H. Mollendal, J. Mol. Struct. 18, 247 (1973).

J. Phys. Chem. Ref. Datt, Vol. 8, No. 3, 1979

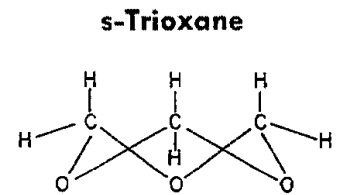

$\mathrm{C}_{3} \mathrm{H}_{6} \mathrm{O}_{3}$

\begin{tabular}{cc|cc}
\hline \hline Bond & Substitution & Angle & Substitution \\
\hline \multirow{2}{*}{ CO } & $1.420 \mathrm{C}$ & OCO & $112.0 \mathrm{C}$ \\
& & COC & $109.5 \mathrm{C}$
\end{tabular}

[1] J. M. Colmont, J. Mol. Struct. 21, 387 (1974).

[2] T. Oka, K. Tsuchiya, S. Iwata and Y. Morino, Bull. Chem. Soc. Japan 37, 4 (1964).

\section{Methyl Vinyl Sulfide}

$\mathrm{C}_{3} \mathrm{H}_{6} \mathrm{~S}$

$\mathrm{CH}_{3} \mathrm{SCH}=\mathrm{CH}_{2}$

$\mathrm{C}_{\sharp}$

Cis conformation (with planar heavy atoms) was definitely established.

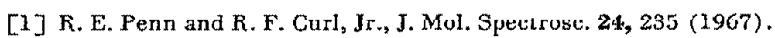

2-Propene-1-thiol (Allyl Mercaptan)

$\mathrm{C}_{3} \mathrm{H}_{6} \mathrm{~S}$

$\mathrm{CH}_{2}=\mathrm{CHCH}_{2} \mathrm{SH}$

$\mathrm{C}_{1}$

\begin{tabular}{cc}
\hline \hline Angle & Effective \\
\hline$\alpha$ & $124 \mathrm{X}$ \\
$\beta$ & $50 \mathrm{X}$
\end{tabular}

Only gauche form exists. With $\alpha$ and $\beta$ defined as zero in hypo. thetical cis form, the observed gauche form is estimated to have the above values of $\alpha$ and $\beta$, the sense being such that vinyl and thiol groups, respectively, are rotated abuve the plane of the paper.

[1] K, V. L. N. Sastry, S. C. Dass, W. V. F. Brooks, and A. Bhaumitt, J. Mol. Spectrosc. 31, 54 (1969).

\section{Selenetane (Trimethylene Selenide)}

\begin{tabular}{lll}
$\mathrm{C}_{3} \mathrm{H}_{6} \mathrm{Se}$ & $\stackrel{\mathrm{CH}_{2}-\mathrm{CH}_{2}-\mathrm{CH}_{2}-\mathrm{Se}}{\mathrm{C}}$ \\
\hline \hline Angle & Effective \\
\hline Dihedral & $29.5 \mathrm{X}$
\end{tabular}

Various assumptions about vibrational motion required to obtain dihedral angle estimate.

[1] M. G. Petit, J. S. Gibson, and D. O. Harris, J. Chem. Phys. 53, $3408(1970)$. 


\section{2-Bromopropane}

\begin{tabular}{cc|cc}
$\mathrm{C}_{3} \mathrm{H}_{7} \mathrm{Br}$ & \multicolumn{2}{c}{$\mathrm{CH}_{2} \mathrm{CHBrCH}_{3}$} & $\mathrm{C}_{*}$ \\
\hline Bond & Substitution & Angle & Substitution \\
\hline $\mathrm{CBr}$ & $1.957 \mathrm{~B}$ & $\mathrm{CCBr}$ & $110.0 \mathrm{~B}$ \\
$\mathrm{CC}$ & $1.508 \mathrm{D}$ & $\mathrm{CCC}$ & $114.2 \mathrm{C}$
\end{tabular}

[1] 11. II. Soldwendenan and F, L. Tobiason, J. Chom. Phys. 48, 201 (1965).

\section{2-Chloropropane}

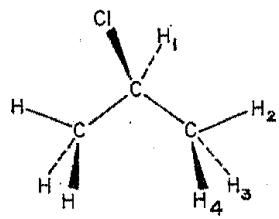

$\mathrm{C}_{3} \mathrm{H}_{7} \mathrm{Cl}$

\begin{tabular}{ll|ll}
\hline \hline Bond & Substitution & Angle & Substitution \\
\hline & & & \\
& $1.798 \mathrm{C}$ & $\mathrm{CCC}$ & $112.5 \mathrm{C}$ \\
$\mathrm{CC}$ & $1.523 \mathrm{C}$ & $\mathrm{CCCl}$ & $109.3 \mathrm{~B}$ \\
$\mathrm{CH}{ }_{1}$ & $1.091 \mathrm{~B}$ & $\mathrm{CCH}$ & $110.1 \mathrm{~B}$ \\
$\mathrm{CH}_{2,3,4}$ & $1.092 \mathrm{C}$ & $\mathrm{ClCH}_{1}$ & $105.4 \mathrm{C}$ \\
& & $\mathrm{CCH}_{2}$ & $110.7 \mathrm{C}$ \\
& & $\mathrm{CCH}_{3,4}$ & $109.7 \mathrm{C}$ \\
& & $\mathrm{H}_{3} \mathrm{CH}_{4}$ & $109.1 \mathrm{C}$ \\
& & $\mathrm{H}_{2} \mathrm{CH}_{3}$ & $109.1 \mathrm{C}$ \\
& & $\mathrm{H}_{2} \mathrm{CH}_{4}$ & $108.7 \mathrm{C}$ \\
\hline
\end{tabular}

[1] F. L. Tobiason and R. H. Schwendeman, Y. Chem. Phys. 40, 1014 (1964).
Cyclopropaneamine

(Cyclopropylamine)

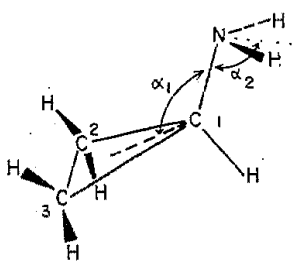

$\mathrm{C}_{3} \mathrm{H}_{7} \mathrm{~N}$

$\mathrm{C}_{\mathrm{s}}$

\begin{tabular}{lll|lll}
\hline \hline Bond Substitution & Effective $^{\mathrm{a}}$ & Angle & Substitution & Effective \\
\hline & & & & & \\
$\mathrm{C}_{1} \mathrm{C}_{2}$ & $1.486 \mathrm{C}$ & & $\mathrm{C}_{2} \mathrm{C}_{1} \mathrm{C}_{3}$ & $61.2 \mathrm{~B}$ & \\
$\mathrm{C}_{2} \mathrm{C}_{3}$ & $1.513 \mathrm{~B}$ & & $\mathrm{CCN}$ & & $116.3 \mathrm{C}$ \\
$\mathrm{CN}$ & & $1.462 \mathrm{D}$ & $\mathrm{CNH}$ & & $111.0 \mathrm{E}$ \\
$\mathrm{NH}$ & & $1.008 \mathrm{D}$ & $\mathrm{HNH}$ & & $108.1 \mathrm{D}$ \\
$\mathrm{H} \cdots \mathrm{H}$ & $1.632 \mathrm{~B}$ & & $\alpha_{1}$ & & $121.0 \mathrm{C}$ \\
& & & $\alpha_{2}$ & & $127.5 \mathrm{X}$ \\
& & & & \\
\hline
\end{tabular}

${ }^{2}$ All CH parameters were assumed to obtain these values. Un certainties reflect a reasonable range for the assumed parameters.

[1] D. K. Hendricksen and M. D. Harmony, J. Chem. Phys. 51, $700(1969)$.

[2] M. D. Harmony, R. E. Bostrom and D. K. Hendricksen, J. Chem. Phys. 62, 1599 (1975).

[3] M. D. Harmony, personal communication.

\section{2-Methylaziridine (trans-Propyleneimine)}

$\mathrm{C}_{3} \mathrm{H}_{3} \mathrm{~N}$

$$
\mathrm{HN}-\mathrm{CH}_{2}-\mathrm{CH}-\mathrm{CH}_{3}
$$

Methyl group trans to hydrogen on nitrogen.

[1] Y. S. L.i. M. D. Harmony, D. Hayes, and E. T.. Beeson, Ir.. I. Chem. Phys. 47, 4514 (1967).

\section{Cyclopropylphosphine}

$\mathrm{C}_{3} \mathrm{H}_{7} \mathrm{P}$

$$
\mathrm{CH}_{2}-\mathrm{CHI}_{2}-\mathrm{CHPH}_{2}
$$

$\mathrm{C}_{s}$

$\mathrm{C}_{\mathrm{s}}$ conformation confirmed; see cyclopropylamine.

[1] L. A. Dinsmore, C. O. Britt, and J. E. Boggs, J. Chem. Phys. 54, 915 (1971). 
Propane

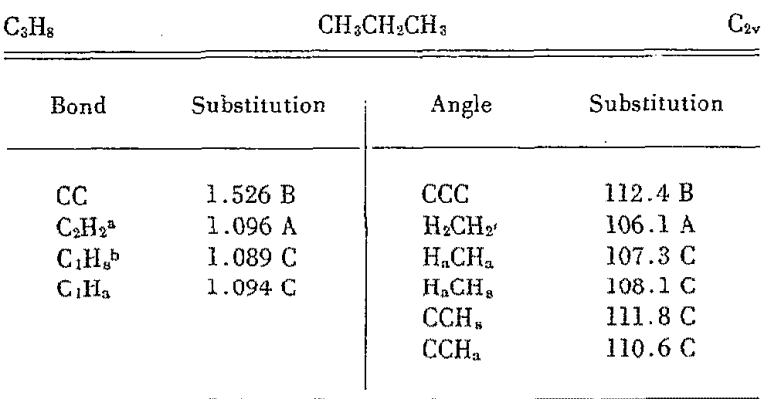

a $\mathrm{C}_{9}$ is methylene carbon.

b $\mathrm{H}_{8}$ and $\mathrm{H}_{\mathrm{a}}$ are in-plane and out-of-plane, respectively.

[1] D. R. Lide, Jr., J. Chem. Phys. 33, 1514 (1960).

\section{trans-Ethylmethylether}

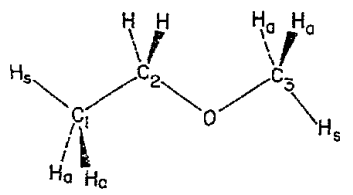

$\mathrm{C}_{3} \mathrm{H}_{8} \mathrm{O}$

\begin{tabular}{ll|ll}
\hline \hline Bond & Substztution & Angle & Substitution \\
\hline & & & \\
$\mathrm{C}_{1} \mathrm{C}_{2}$ & $1.520 \mathrm{~B}$ & $\mathrm{C}_{2} \mathrm{OC}_{3}$ & $111.8 \mathrm{C}$ \\
$\mathrm{C}_{2} \mathrm{O}$ & $1.404 \mathrm{C}$ & $\mathrm{C}_{1} \mathrm{C}_{2} \mathrm{O}$ & $108.2 \mathrm{C}$ \\
$\mathrm{C}_{3} \mathrm{O}$ & $1.415 \mathrm{C}$ & $\mathrm{HC}_{2} \mathrm{H}$ & $107.2 \mathrm{~B}$ \\
$\mathrm{C}_{2} \mathrm{H}$ & $1.101 \mathrm{~B}$ & $\mathrm{C}_{1} \mathrm{C}_{2} \mathrm{H}$ & $110.9 \mathrm{~B}$ \\
$\mathrm{C}_{3} \mathrm{H}_{8}$ & $1.084 \mathrm{C}$ & $\mathrm{OC}_{3} \mathrm{H}_{3}$ & $107.7 \mathrm{~B}$ \\
$\mathrm{C}_{3} \mathrm{H}_{\mathrm{n}}$ & $1.100 \mathrm{~B}$ & $\mathrm{OC}_{3} \mathrm{H}_{8}$ & $111.0 \mathrm{C}$ \\
$\mathrm{C}_{3} \mathrm{H}_{3}$ & $1.039 \mathrm{C}$ & $\mathrm{H}_{2} \mathrm{C}_{3} \mathrm{H}_{3}$ & $108.4 \mathrm{~B}$ \\
$\mathrm{C}_{1} \mathrm{H}_{3}$ & $1.092 \mathrm{~B}$ & $\mathrm{C}_{2} \mathrm{C}_{1} \mathrm{H}_{3}$ & $110.5 \mathrm{~B}$ \\
& & $\mathrm{C}_{2} \mathrm{C}_{1} \mathrm{H}_{3}$ & $110.1 \mathrm{~B}$ \\
& & $\mathrm{H}_{3} \mathrm{C}_{1} \mathrm{H}_{2}$ & $108.6 \mathrm{~B}$
\end{tabular}

[1] M. Hayashi and K. Kuwada, J. Mol. Struct. 28, 147 (1975).

\section{n-Propyl Alcohol}

$\mathrm{C}_{3} \mathrm{H}_{8} \mathrm{O}$

$\mathrm{CH}_{3} \mathrm{CH}_{2} \mathrm{CH}_{2} \mathrm{OH}$

Both trans and gauche forms exist. Insufficient data for structural determination.

[1] L. M. Imanov, A. A. Abdurakhmanov and R. A. Ragimova, Opt. and Spectrosc. 25, 528 (1968).

[2] A. A. Abdurakhmanov, R. A. Ragimova and L. M. Imanov, Opt. and Spectrosc. 26, 75 (1969).

\section{2-Methoxyethanol}

$\mathrm{C}_{3} \mathrm{H}_{8} \mathrm{O}_{2}$

$\mathrm{CH}_{3}-\mathrm{O}-\mathrm{CH}_{2}-\mathrm{CH}_{2}-\mathrm{OH}$

$\mathrm{C}_{1}$

Conformation has been shown to be gauche about each of the $\mathrm{CH}_{3} \mathrm{O}-\mathrm{C}, \mathrm{C}-\mathrm{C}$ and $\mathrm{C}-\mathrm{OH}$ bonds.

[1] P. Buckley and M. Brochu, Can. J. Chem. 50, 1149 (1972).

\section{Trimethylarsine}

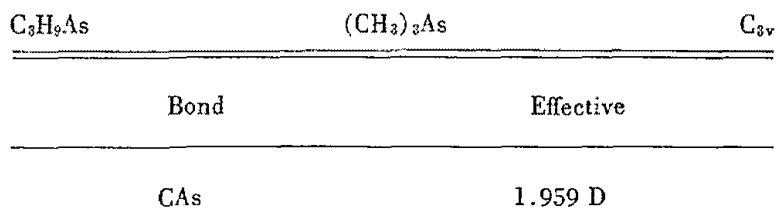

Assumptions concerning angle $\mathrm{CAsC}$ and methyl group parameters have a relatively small effect upon the $\mathrm{CA}$ s distance.

[1] D. R. Lide, Spectrochim. Acta, 1959, 473 (1959).

Trimethylamine-Boron Trifluoride

\begin{tabular}{ll} 
Bond & $\left(\mathrm{CH}_{3}\right)_{3} \mathrm{NBF}_{3}$ \\
\hline BN & $1.636 \mathrm{~B}$ \\
\hline L P. S. Bryan and R. L. Kuczkowski, Inorg. Chem. 10, 200 (1971).
\end{tabular}

\section{Bromotrimethylgermane}

\begin{tabular}{|c|c|c|c|c|}
\hline \multicolumn{2}{|l|}{$\mathrm{C}_{3} \mathrm{H}_{9} \mathrm{BrGe}$} & \multicolumn{2}{|c|}{$\left(\mathrm{CH}_{3}\right)_{3} \mathrm{GeBr}$} & $\mathrm{C}_{3 \mathrm{v}}$ \\
\hline Bond & Substitution & Effective & Angle & Effective \\
\hline $\begin{array}{l}\mathrm{GeBr} \\
\mathrm{GeC}\end{array}$ & $2.323 \mathrm{~A}$ & $1.936 \mathrm{C}$ & $\mathrm{BrGeC}^{\mathrm{a}}$ & $106.3 \mathrm{C}$ \\
\hline
\end{tabular}

a Methyl group parameters were assumed to obtain this parameter. [1] Y. S. Li and J. R. Durig, Inorg. Chem. 12, 306 (1973)

\section{Trimethylchlorogermane}

\begin{tabular}{|c|c|c|c|c|}
\hline $\mathrm{C}_{3} \mathrm{H}_{9} \mathrm{ClGe}$ & & $\left(\mathrm{CH}_{3}\right)_{3} \mathrm{Ge}$ & & $\mathrm{C}_{3 v}$ \\
\hline Bond & Substitution & Effective & Angle & Effective \\
\hline $\begin{array}{l}\mathrm{GeCl} \\
\mathrm{GeC}\end{array}$ & $2.170 \mathrm{~A}$ & $1.940 \mathrm{~B}$ & $\mathrm{CGeCl}$ & $105.9 \mathrm{~B}$ \\
\hline
\end{tabular}

Methyl group parameters were assumed $(\mathrm{CH}=1.095, \mathrm{HCH}=$ 109.5) to obtain effective parameters.

[I] J. R. Durig and K. L. Hellams, J. Mol. Struct. 29, 349 (1975). 
Chlorotrimethylsilane

\section{(Trimethylchlorosilane)}

\begin{tabular}{ll|ll}
$\mathrm{C}_{3} \mathrm{H}_{9} \mathrm{ClSi}$ & & $\left(\mathrm{CH}_{3}\right)_{3} \mathrm{SiCl}$ & $\mathrm{C}_{3 \mathrm{v}}$ \\
\hline \hline Bond & Effective & Angle & Effective \\
\hline $\mathrm{SiCl}$ & $\begin{array}{l}2.022 \mathrm{E} \\
\mathrm{SiC}\end{array}$ & $\mathrm{ClsiC}$ & $110.5 \mathrm{D}$ \\
& & & \\
\hline
\end{tabular}

Methyl group parameters were assumed in order to obtain the other parameters.

[1] J. R. Durig, R. O. Carter, and Y. S. Li, J. Mol. Spectrusc. 44, $18(1972)$

\section{2-Aminopropane}<smiles>CC(C)N</smiles>

$\mathrm{C}_{3} \mathrm{H}_{9} \mathrm{~N}$

Isotopic substitution showed conclusively that. amino group occupied the trans symmetrical position.

[1] S. C. Mehrota, L. L. Griffin, C. 0. Britt and J. E. Boggs, J. Mol. Spectrosc. 64, 244 (1977).

\section{Trimethylamine}

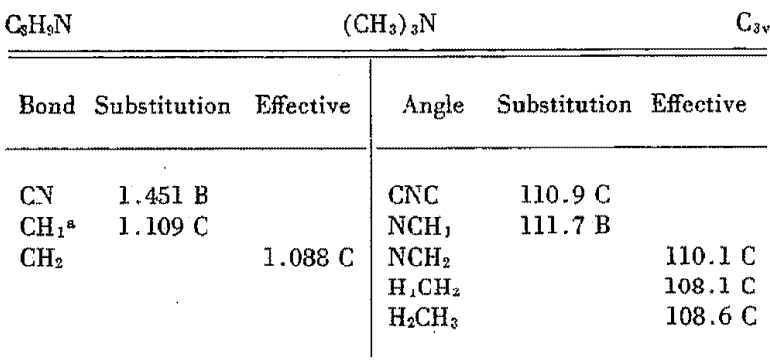

The $\mathrm{H}_{2} \mathrm{H}_{3}$ distance was assumed to be $1.7668 \AA$. The methyl groups are tilted $1.3^{\circ}$ toward the nitrogen lone pair.

$\therefore \mathrm{H}_{1}$ lies in plane of symmetry; $\mathrm{H}_{2}$ and $\mathrm{H}_{3}$ are symmetrically situated out of plane.

[1] J. E. Wollrab and V. W. Laurie, J. Chem. Phys. 51, 1580 (1969).

[2] D. R. Lide, Jr., and D. E. Mann, J. Chem. Phys. 28, 572 (1958).

\section{1-Amino-2-propanol}

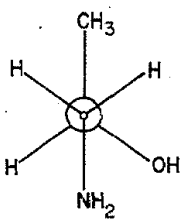

$\mathrm{C}_{3} \mathrm{H}_{3} \mathrm{NO}$

The microwave data were consistent with an intramolecularly hydrogen-bonded conformation as shown.

[1] K. M. Marstokk and H. Mollendal, J. Mol. Struct. 35, 57 (1976).

Trimethylphosphine

\begin{tabular}{|c|c|c|c|c|}
\hline Bond & Substitution & Effective & Angle & Effective \\
\hline $\begin{array}{l}\mathrm{CH}_{\mathrm{s}}{ }^{\mathrm{a}} \\
\mathrm{CH}_{\mathrm{a}}{ }^{\mathrm{a}} \\
\mathrm{PC}\end{array}$ & $1.112 \mathrm{D}$ & $\begin{array}{l}1.090 \mathrm{D} \\
1.843 \mathrm{C}\end{array}$ & $\begin{array}{l}\mathrm{PCH}_{\mathrm{g}} \\
\mathrm{PCH}_{\mathrm{a}} \\
\mathrm{H}_{8} \mathrm{CH}_{9} \\
\mathrm{H}_{\mathrm{a}} \mathrm{CH}_{\mathrm{g}^{\prime}} \\
\mathrm{CPC}\end{array}$ & $\begin{array}{r}111.4 \mathrm{D} \\
109.8 \mathrm{D} \\
108.2 \mathrm{D} \\
109.4 \mathrm{D} \\
98.9 \mathrm{C}\end{array}$ \\
\hline
\end{tabular}

a $s$ refers to in plare, a to out of plane.

[1] P. S. Bryan and R. L. Kuczkowski, J. Chem. Phys. 55, 3049 (1971).

\section{Trimethylgermane}

\begin{tabular}{lcc|cc}
$\mathrm{C}_{3} \mathrm{H}_{10} \mathrm{Ge}$ & & $\left(\mathrm{CH}_{3}\right)_{3} \mathrm{GeH}$ & $: \mathrm{C}_{3 \mathrm{v}}$ \\
\hline \hline Bond & Substitution & Effective & Angle & Effective \\
\hline $\begin{array}{l}\mathrm{GeH} \\
6 \mathrm{GeC}\end{array}$ & $1.522 \mathrm{C}$ & & & \\
\hline
\end{tabular}

Methyl group parameters were assumed to obtain these parameters.

[1] J. R. Durig, M. M. Chen, Y. S. I,i, and J. B. Turner, J. Phys. Chem. 77, 227 (1973).

\section{Trimethylsilane}

\begin{tabular}{lll|lll}
$\mathrm{C}_{3} \mathrm{H}_{10} \mathrm{Si}$ & $\left(\mathrm{CH}_{3}\right)_{3} \mathrm{SiH}$ & $\mathrm{C}$ \\
\hline \hline Bond & Substitution & Effective & Angle & Substitution & Effective \\
\hline $\mathrm{SiC}$ & $1.868 \mathrm{~B}$ & & $\mathrm{CSiC}$ & $110.2 \mathrm{~B}$ & \\
$\mathrm{SiH}$ & $1.489 \mathrm{~B}$ & & $\mathrm{HCH}$ & & $107.9 \mathrm{C}$ \\
$\mathrm{CH}$ & & $1.095 \mathrm{C}$ & & & \\
\hline
\end{tabular}

The methyl groups are staggered with respect to the SiH bond, and have been assumed to be symmetric about the $\mathrm{SiC}$ bond.

[1] L. Pierce and D. H. Petersen, J. Chem. Phys. 33, 907 (1960). 
Trimethylamineborane

\begin{tabular}{ll|ll}
$\mathrm{C}_{3} \mathrm{H}_{13} \mathrm{BN}$ & $\left(\mathrm{CH}_{8}\right)_{3} \mathrm{NBH}_{3}$ & $\mathrm{C}_{3 \mathrm{v}}$ \\
\hline \hline Bond & Substitution & Angle & Substitution \\
\hline $\mathrm{BN}$ & $1.638 \mathrm{C}$ & $\mathrm{CNB}$ & $109.9 \mathrm{C}$ \\
$\mathrm{CN}$ & $1.483 \mathrm{C}$ & $\mathrm{NBH}$ & $105.3 \mathrm{C}$ \\
$\mathrm{BH}$ & $1.211 \mathrm{C}$ & & \\
\hline
\end{tabular}

[1] P. Cassoux, R. L. Kuczkowski, P. S. Bryan, and R. C. Taylor, Inorg. Chem, 14, 126 (1975).

[2] J. R. Durig, Y. S. Li, and J. Odom, J. Mol. Struct. 16, 443 (1973).

[1] H. G. Schirdewahn, Doctoral Thesis, University of Freiburg, 1965.

Trimethylphosphineborane

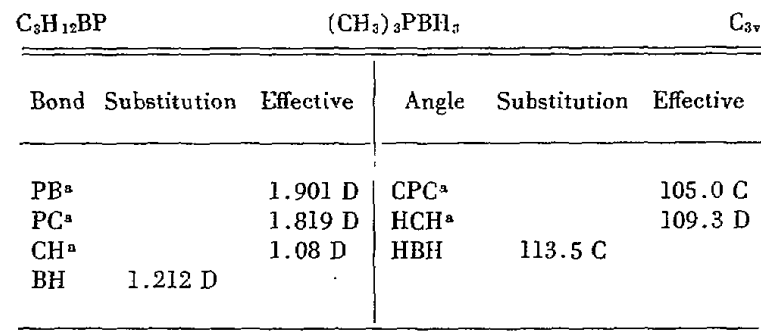

- In order to obtain these parameters, symmetrical $\mathrm{CH}_{3}$ groups and the $\mathrm{H}$....H distance in the methyl groups were assumed. [1] P. S. Bryan and R. L. Kuczkowski, Inorg. Chem. 11, 553 (1972).

\section{lodocyanoacefylene}

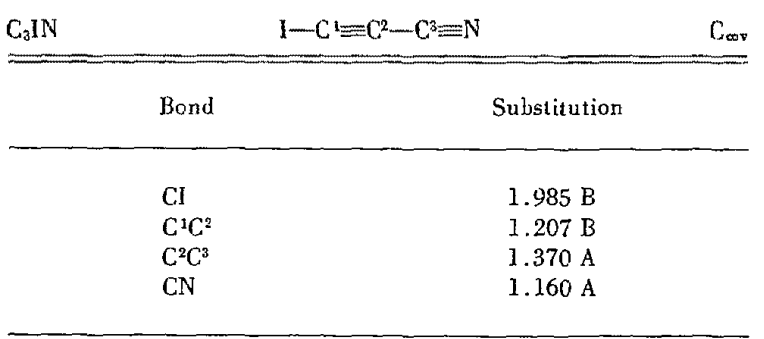

[1] T. Bjorvatten, J. Mol. Struct. 20, 75 (1974).

\section{$\mathrm{C}_{4}$ Molecules}

\section{Butadiyne (Diacetylene)}

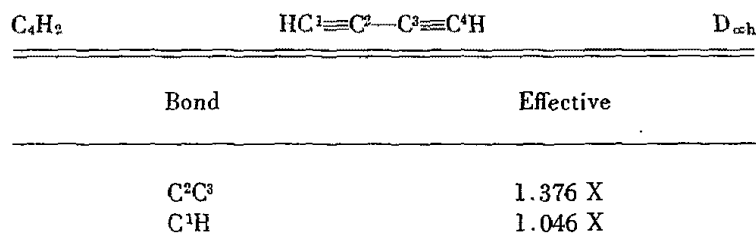

In order to calculate the structure it was necessary to assume the $\mathrm{C} \equiv \mathrm{C}$ bond distance. The structural parameters listed above are rated $\mathrm{X}$ because of the uncertainty arising from this assumption.

[1] J. H. Callomon and B. P. Stoicheff, Can. J. Phys. 35, 373 (1957).

\section{1,1,1-Trifluara-2-butyne}

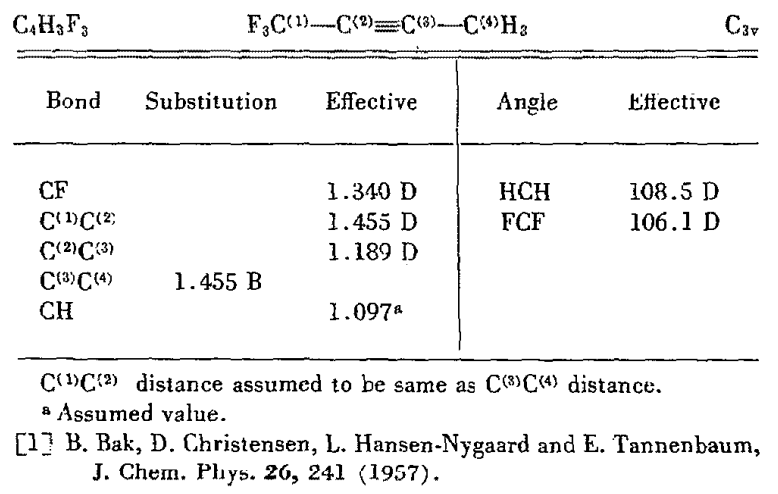

\section{2-Butynenitrile \\ (Methylcyanoacelylene)}

$\mathrm{C}_{4} \mathrm{H}_{3} \mathrm{~N} \quad \mathrm{CH}_{3}-\mathrm{C} \equiv \mathrm{C}-\mathrm{CN} \quad \mathrm{C}_{3 \mathrm{v}}$

Molecular symmetry confirmed.

[1] J. Sheridan and L. F. Thomas, Nature 174, 798 (1954).

$\underbrace{\mathrm{C}_{4} \mathrm{H}_{4}}_{\mathrm{C}^{2} \mathrm{C}^{3}} \underbrace{\mathrm{C}^{3} \mathrm{H}_{2}=\mathrm{C}^{2}=\mathrm{C}^{3}=\mathrm{C}^{4} \mathrm{H}_{2}}_{\text {Effective }} \underbrace{\mathrm{D}_{2 \mathrm{~b}}}_{1.284 \mathrm{X}}$

In order to derive the $\mathrm{C}^{2} \mathrm{C}^{3}$ bond distance it was necessary to assume $\mathrm{HCH}_{3} \mathrm{C}^{3} \mathrm{C}^{4}$, and $\mathrm{C}^{1} \mathrm{H}$. Because of the assumptions an estimate of the uncertainty in $\mathrm{C}^{2} \mathrm{C}^{3}$ can not be derived.

[1] B. P. Stoicheff, Can. J. Phys. \$5, 837 (1957). 
Trifluoroacetic Acid-Monofluoroacetic Acid Dimer

$\mathrm{C}_{4} \mathrm{H}_{4} \mathrm{~F}_{4} \mathrm{O}$

\begin{tabular}{cc}
\hline Bond & Effective \\
\hline $0 \cdots 0$ & $2.69 \times$
\end{tabular}

$\lceil 17$ C. C. Costain and G. P. Srivastava. J. Chem. Phys. 41, 1620 (1964).

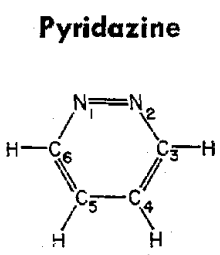

$\mathrm{C}_{4} \mathrm{H}_{4} \mathrm{~N}_{2}$

\begin{tabular}{ll|ll}
\hline Bond & Substitution & Angle & Substitution \\
\hline $\mathrm{NN}$ & $1.330 \mathrm{~A}$ & $\mathrm{NNC}$ & $119.0 \mathrm{E}$ \\
$\mathrm{NC}$ & $1.353 \mathrm{E}$ & $\mathrm{NCC}$ & $123.7 \mathrm{E}$ \\
$\mathrm{C}_{3} \mathrm{C}_{4}$ & $1.382 \mathrm{E}$ & $\mathrm{CCC}$ & $117.3 \mathrm{E}$ \\
$\mathrm{C}_{4} \mathrm{C}_{3}$ & $1.375 \mathrm{~A}$ & & \\
\hline
\end{tabular}

[1] W. Werner, H. Dreizler, and H. D. Rudolph, Z. Naturforsch. 22A, 531 (1967).

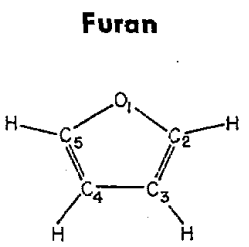

\begin{tabular}{|c|c|c|c|}
\hline Bond & Substitution & Angle & Substitution \\
\hline $\mathrm{CO}$ & $1.362 \mathrm{~B}$ & $\mathrm{C}_{5} \mathrm{OC}_{2}$ & $106.5 \mathrm{~B}$ \\
\hline $\mathrm{C}_{2} \mathrm{C}_{3}$ & $1.36 \mathrm{lB}$ & $O \mathrm{C}_{2} \mathrm{C}_{3}$ & $110.7 \mathrm{~B}$ \\
\hline $\mathrm{C}_{3} \mathrm{C}_{4}$ & $1.431 \mathrm{~A}$ & $\mathrm{C}_{2} \mathrm{C}_{3} \mathrm{C}_{4}$ & $106.0 \mathrm{~B}$ \\
\hline $\mathrm{C}_{2} \mathrm{H}$ & $1.075 \mathrm{~B}$ & $\mathrm{OC}_{2} \mathrm{H}$ & $115.9 \mathrm{~B}$ \\
\hline $\mathrm{C}_{3} \mathrm{H}$ & $1.0 \% \mathrm{~A}$ & $\mathrm{C}_{4} \mathrm{C}_{3} \mathrm{H}$ & $127.9 \mathrm{~A}$ \\
\hline
\end{tabular}

[1] B. Bak, D. Christensen, W. B. Dixon, L. Hansen-Nygaard, J. R. Andersen, and M. Schottländer, J. Mol. Spectrose. 9, 124 (1962).
Thiophene

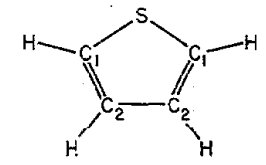

\begin{tabular}{|c|c|c|c|}
\hline Bond & Substitution & Angle & Substitution \\
\hline $\mathrm{SC}$ & $1.714 \mathrm{~B}$. & $\csc$ & $92.1 \mathrm{~A}$ \\
\hline $\mathrm{C}_{1} \mathrm{C}_{2}$ & I. $369 \mathrm{~B}$ & $\mathrm{SC}_{1} \mathrm{C}_{2}$ & $111.5 \mathrm{~A}$ \\
\hline $\mathrm{C}_{2} \mathrm{C}_{2}$ & 1. $423 \mathrm{~B}$ & $\mathrm{C}_{1} \mathrm{C}_{2} \mathrm{C}_{2}$ & $112.5 \mathrm{~A}$ \\
\hline $\mathrm{C}_{1} \mathrm{H}$ & $1.078 \mathrm{~B}$ & $\mathrm{SCH}$ & $119.8 \mathrm{~B}$ \\
\hline $\mathrm{C}_{2} \mathrm{H}$ & $1.080 \mathrm{~B}$ & $\mathrm{C}_{2} \mathrm{C}_{2} \mathrm{H}$ & $124.3 \mathrm{~A}$ \\
\hline
\end{tabular}

[1] B. Bak, D. Christensen, L. Hansen-Nygaard and J. RastrupAndersen, J. Mol. Spectrosc. 7, 58 (1961).

[2] B. Bak, D. Christensen, J. Rastrup-Andersen and E. Tannenbaum, J. Chem. Phys. 25, 892 (1956).

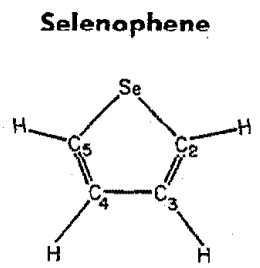

$\mathrm{C}_{4} \mathrm{H}_{4} \mathrm{Se}$

\begin{tabular}{lc|cr}
\hline Bond & Substitution & Angle & Substitution \\
\hline & & & \\
$\mathrm{SeC}_{2}$ & $1.855 \mathrm{~A}$ & $\mathrm{C}_{5} \mathrm{SeC}_{2}$ & $87.8 \mathrm{~B}$ \\
$\mathrm{C}_{2} \mathrm{C}_{3}$ & $1.369 \mathrm{~B}$ & $\mathrm{SeC}_{2} \mathrm{C}_{3}$ & $111.6 \mathrm{~B}$ \\
$\mathrm{C}_{3} \mathrm{C}_{4}$ & $1.433 \mathrm{~B}$ & $\mathrm{C}_{2} \mathrm{C}_{3} \mathrm{C}_{4}$ & $114.6 \mathrm{~B}$ \\
$\mathrm{C}_{2} \mathrm{H}$ & $1.070 \mathrm{~B}$ & $\mathrm{SeC}_{2} \mathrm{H}_{2}$ & $121.7 \mathrm{~B}$ \\
$\mathrm{C}_{3} \mathrm{H}$ & $1.079 \mathrm{~B}$ & $\mathrm{C}_{4} \mathrm{C}_{3} \mathrm{H}_{3}$ & $122.9 \mathrm{~B}$ \\
\hline
\end{tabular}

[1] N. M. Posdeev: O. B. Akulinin, A. A. Shaplin and N. N. Magdesieva, J. Struct. Chem. 11, 804 (1970). 
1-Chloro-2-butyne

\begin{tabular}{ll|ll}
$\mathrm{C}_{4} \mathrm{H}_{5} \mathrm{Cl}$ & \multicolumn{2}{c}{$\mathrm{ClH}_{2} \mathrm{C}-\mathrm{C}=\mathrm{C}-\mathrm{CH}_{3}$} & $\mathrm{C}_{\mathrm{B}}$ \\
\hline \hline \multirow{2}{*}{ Bond } & Effective & Angle & Effective \\
\hline & & & \\
$\mathrm{CH}^{\mathrm{s}}$ & $1.110^{\mathrm{a}}$ & $\mathrm{HCH}^{\mathrm{a}}$ & $108.5^{\circ}$ \\
$\mathrm{CH}^{\mathrm{b}}$ & $1.090^{\circ}$ & $\mathrm{HCH}^{\mathrm{b}}$ & $108.5^{\circ}$ \\
$\mathrm{C} \equiv \mathrm{C}$ & $1.207^{\mathrm{a}}$ & $\mathrm{CCCl}$ & $111.5 \mathrm{X}$ \\
$\mathrm{CC}$ & $1.458 \mathrm{X}$ & & \\
$\mathrm{CC}$ & $1.460 \mathrm{X}$ & & \\
$\mathrm{CCl}$ & $1.798 \mathrm{X}$ & & \\
\hline
\end{tabular}

a Refers to $\mathrm{CH}_{3}$ portion of molecule.

b Refers to $\mathrm{CH}_{2} \mathrm{Cl}$ portion of molecule.

- These values were assumed.

[1] V. W. Laurie and D. R. Lide, J. Chem. Phys. 31, 939 (1959).

\section{Cyclopropanecarboxylic acid chloride}

$\mathrm{C}_{4} \mathrm{H}_{5} \mathrm{ClO}$

$\left(\mathrm{C}_{3} \mathrm{H}_{8}\right) \mathrm{COCl}$

c.

The $\mathrm{COCl}$ group bisects the ring plane and has the $\mathrm{Cl}$ atom cis to the ring hydrogen.

[1] K. P. R. Nair and J. E. Boggs, J. Mol. Struct. 33, 45 (1976).

Trifluoroacetic Acid-Acetic Acid Dimer

\begin{tabular}{l}
$\mathrm{C}_{4} \mathrm{H}_{5} \mathrm{~F}_{3} \mathrm{O}_{4} \quad \mathrm{CF}_{3} \mathrm{CO}_{2} \mathrm{H} \cdot \mathrm{CH}_{3} \mathrm{CO}_{2} \mathrm{H}$ \\
\hline
\end{tabular}

\begin{tabular}{cc}
\hline \hline Bond & Effective \\
\hline $0 \cdots 0$ & $2.67 \times$
\end{tabular}

[1] C. C. Costain and G. P. Srivastava, J. Chem. Phys. 41, 1620 (1964).

\section{Butenonitrilo \\ (Allyl Cyanide)}

$\mathrm{C}_{6} \mathrm{H}_{5} \mathrm{~N}$

Two rotational isomers, "cis" and "gauche" were identified. No further structural analyses were performed.

[1] K. V. L. N. Sastry, V. M. Rao and S. C. Dass, Can. J. Physics 46,959 (1968).

\section{Cyclopropanecarbonitrile}

(Cyclopropylcyanide)

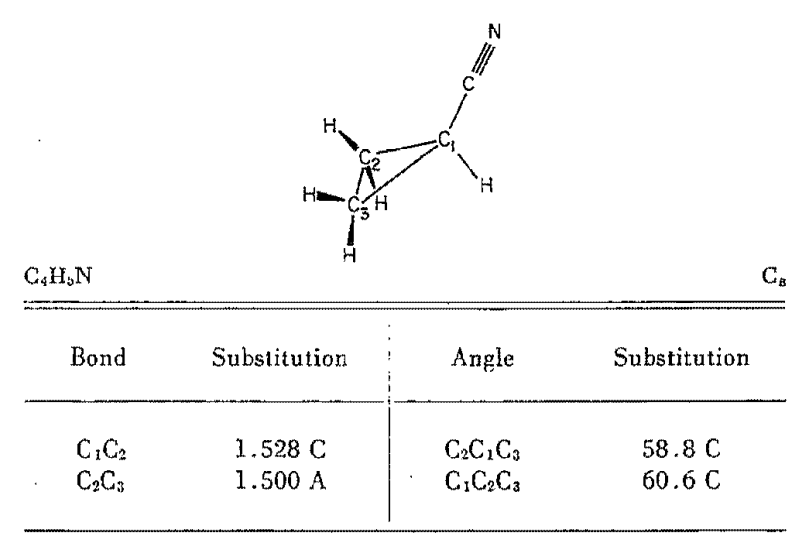

[1] R. Pearson, Jr., A. Choplin and V. W. Laurie, J. Chem. Phys. 624859 (1975).

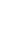

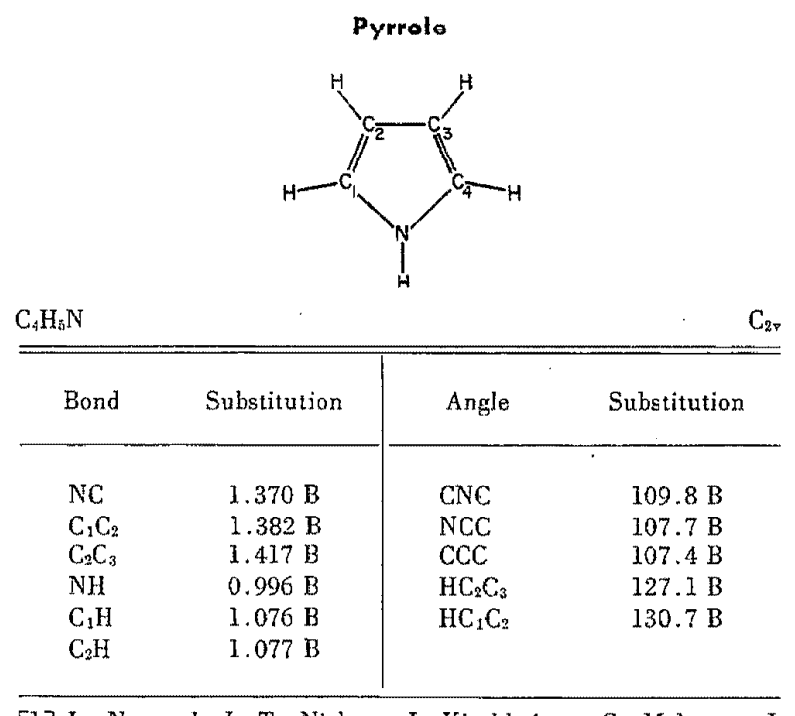

[1] L. Nygaard, J. T. Nielsen, J. Kirchheiner, G. Maltesen, J. Rastrup-Andersen and G. O. Sorenson, J. Mol. Struct. 3, 491 (1969),

[2] B. Bak, D. Christensen, L. Hansen and J. Rastrup-Andersen, J. Chem. Phys. 24, 720 (1956). 
Bicyclo[1.1.0]butane

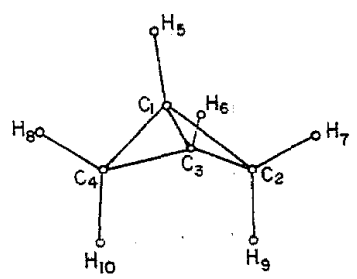

$\mathrm{C}_{4} \mathrm{H}_{5}$

\begin{tabular}{ll|lr}
\hline \hline Bond & Substitution & Angle & Substitution \\
\hline & & & \\
$\mathrm{C}_{3} \mathrm{C}_{3}$ & $1.497 \mathrm{~B}$ & $\mathrm{C}_{1} \mathrm{C}_{3} \mathrm{C}_{2}$ & $60.0 \mathrm{~A}$ \\
$\mathrm{C}_{2} \mathrm{C}_{3}$ & $\mathrm{i} .498 \mathrm{~B}$ & $\mathrm{C}_{1} \mathrm{C}_{2} \mathrm{C}_{3}$ & $60.0 \mathrm{~A}$ \\
$\mathrm{C}_{2} \mathrm{H}_{9}$ & $1.093 \mathrm{C}$ & $\mathrm{C}_{2} \mathrm{C}_{3} \mathrm{C}_{4}$ & $98.3 \mathrm{~B}$ \\
$\mathrm{C}_{2} \mathrm{H}_{7}$ & $1.093 \mathrm{C}$ & $\mathrm{HCH}$ & $113.6 \mathrm{~B}$ \\
$\mathrm{C}_{1} \mathrm{H}_{5}$ & $1.071 \mathrm{~B}$ & $\mathrm{H}_{9} \mathrm{C}_{2} \mathrm{C}_{3}$ & $116.9 \mathrm{~B}$ \\
& & $\mathrm{H}_{7} \mathrm{C}_{2} \mathrm{C}_{3}$ & $118.1 \mathrm{~B}$ \\
& & $\mathrm{C}_{2} \mathrm{C}_{3} \mathrm{H}_{\mathrm{B}}$ & $129.9 \mathrm{~B}$ \\
& & $\mathrm{C}_{1} \mathrm{C}_{3} \mathrm{H}_{6}$ & $128.4 \mathrm{~B}$ \\
\hline
\end{tabular}

The dihedral angle between the heavy atom planes is $121.7^{\circ}$.

[1] K. W. Cox and M. D. Harmony, J. Chem. Phys. 50, 1976 (1969).

[2] M. D. Harmony and K. W. Cox, J. Amer. Chem. Soc. 88, 5049 (1966).

\begin{tabular}{cccc}
\multicolumn{4}{c}{ trans-1,3-Butadiene } \\
$\mathrm{C}_{4} \mathrm{H}_{6}$ & $\mathrm{C}_{2}=\mathrm{C}_{2}^{2} \mathrm{HC} H=\mathrm{C}^{4} \mathrm{fH}_{2}$ & $\mathrm{C}_{2 \mathrm{~h}}$ \\
\hline Bond & Effective & Angle & Effective \\
\hline $\mathrm{C}^{2} \mathrm{C}^{3}$ & $1.464 \mathrm{X}$ & $\mathrm{C}^{1} \mathrm{C}^{2} \mathrm{C}^{3}$ & $123.2 \mathrm{X}$
\end{tabular}

Because of the assumptions required to derive the above structural parameters, the uncertainties are difficult to evaluate.

[1] A. R. H. Cole, G. M. Mohay and G. A. Osborne, Spectrochim. Acta 23A, 909 (1967).

[2] D. J. Marais, N. Sheppard and B. P. Stoicheff, Tetrahedron 17, 163 (1962).

\section{Cyclobutene}

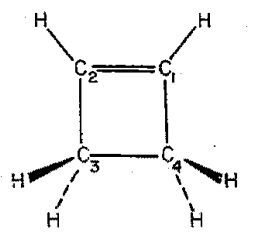

\begin{tabular}{cc|cc}
\multicolumn{1}{c}{$\mathrm{C}_{4} \mathrm{H}_{6}$} & & $\mathrm{C}_{2 \mathrm{r}}$ \\
\hline \hline Bond & Substitution & Angle & Substitution \\
\hline & $1.342 \mathrm{~B}$ & & \\
$\mathrm{C}_{1} \mathrm{C}_{4}$ & $1.517 \mathrm{~B}$ & $\mathrm{C}_{1} \mathrm{C}_{2} \mathrm{C}_{3}$ & $94.2 \mathrm{~B}$ \\
$\mathrm{C}_{1} \mathrm{C}_{4}$ & $\mathrm{C}_{2} \mathrm{C}_{3} \mathrm{C}_{4}$ & $85.8 \mathrm{~B}$ \\
$\mathrm{C}_{3} \mathrm{C}_{4}$ & $1.566 \mathrm{~B}$ & $\mathrm{HC}_{2} \mathrm{C}_{2}$ & $133.5 \mathrm{~B}$ \\
$\mathrm{C}_{1} \mathrm{H}$ & $1.083 \mathrm{~B}$ & $\mathrm{HC}_{3} \mathrm{H}$ & $109.2 \mathrm{~B}$ \\
$\mathrm{C}_{3} \mathrm{H}$ & $1.094 \mathrm{~B}$ & $\mathrm{HC}_{3} \mathrm{C}_{4}$ & $114.5 \mathrm{~B}$ \\
& & $\alpha^{\mathrm{B}}$ & $135.8 \mathrm{~B}$ \\
\hline
\end{tabular}

"Angle between bisector of $\mathrm{HCH}$ and $\mathrm{C}_{3} \mathrm{C}_{4}$ bond.

[I] B. Bak, J. J. Led, L. Nygaard, J. Rastrup-Andersen and G. O. Sorensen, J. Mol. Struct. 3, 369 (1969).

\section{Methylenecyclopropane}

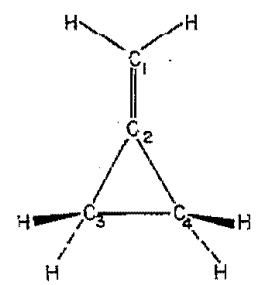

$\mathrm{C}_{4} \mathrm{H}_{6}$

$\mathrm{C}_{34}$

\begin{tabular}{lll|llr}
\hline \hline Bond Substitution & Effective & Angle & Substitution & Effective \\
\hline & & & & & \\
$\mathrm{C}_{1} \mathrm{C}_{2}$ & $1.332 \mathrm{~B}$ & & $\mathrm{C}_{3} \mathrm{C}_{2} \mathrm{C}_{4}$ & $63.9 \mathrm{~B}$ & \\
$\mathrm{C}_{2} \mathrm{C}_{5}$ & $1.457 \mathrm{~B}$ & & $\mathrm{HC}_{1} \mathrm{H}$ & & $114.3 \mathrm{D}$ \\
$\mathrm{C}_{3} \mathrm{C}_{4}$ & $1.542 \mathrm{~A}$ & & $\mathrm{HC}_{3} \mathrm{H}$ & & $113.5 \mathrm{D}$ \\
$\mathrm{C}_{2} \mathrm{HI}$ & & $1.088^{\mathrm{a}}$ & $\alpha^{\mathrm{b}}$ & & $29.2 \mathrm{D}$ \\
$\mathrm{C}_{3} \mathrm{H}$ & & $1.09 \mathrm{D}$ & & & \\
& & & & &
\end{tabular}

Hydrogen parameters based upon assumed value of $\mathrm{C}_{1} \mathrm{H}$.

assumed value.

Angle between $\mathrm{HC}_{3} \mathrm{H}$ bisector and $\mathrm{C}_{3} \mathrm{C}_{4}$ bond axis.

[1] V. W. Laurie and W. M. Stigliani, J. Am. Chem. Soc. 92, 1485 (1970). 
2,3,4,5-Tetracarbahexaborane (6)

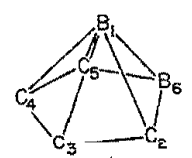

$\mathrm{C}_{4} \mathrm{H}_{6} \mathrm{~B}_{2}$

$\mathrm{C}_{\mathrm{B}}$

\begin{tabular}{ll}
\hline \hline Bond & Substitution \\
\hline $\mathrm{B}_{1} \mathrm{~B}_{6}$ & $1.886 \mathrm{~B}$ \\
$\mathrm{~B}_{1} \mathrm{C}_{2}$ & $1.709 \mathrm{E}$ \\
$\mathrm{B}_{1} \mathrm{C}_{3}$ & $1.697 \mathrm{D}$ \\
$\mathrm{B}_{6} \mathrm{C}_{2}$ & $1.541 \mathrm{C}$ \\
$\mathrm{C}_{2} \mathrm{C}_{3}$ & $1.436 \mathrm{C}$ \\
$\mathrm{C}_{3} \mathrm{C}_{4}$ & $1.424 \mathrm{C}$ \\
\hline
\end{tabular}

Above structure was that obtained by choosing the positive sign for small $C_{2}$ (and $C_{5}$ ) $c$ coordinates. The other choice leads to a less reasonable $\mathrm{B}_{1} \mathrm{C}_{2}$ distance.

[1] J. P. Pasinski and R. A. Beaudet, J. Chem. Phys. 61, 683 (1974).

\section{trans-2-Butenal}

(Crotonaldehyde)

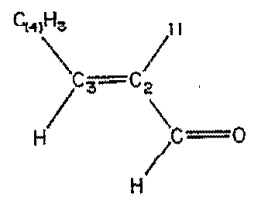

$\mathrm{C}_{4} \mathrm{H}_{6} \mathrm{O}$

$\mathrm{C}_{\mathrm{s}}$

\begin{tabular}{cc}
\hline \hline Angles & Effective \\
\hline $\mathrm{C}_{2} \mathrm{C}_{3} \mathrm{C}_{4}$ & $125.6 \mathrm{X}$ \\
$\mathrm{HC}_{3} \mathrm{C}_{4}$ & $116.1 \mathrm{X}$ \\
\hline
\end{tabular}

a Assumed values used for all other structural parameters.

[1] M. Suzuki and K. Kozima, Bull, Chem. Soc. Japan 42, 2183 (1969).

[2] S. L. Hsu and W. H. Flygare, Chem. Phys. Lett. 4, 317 (1969).

\section{3-Butyn-1-ol}

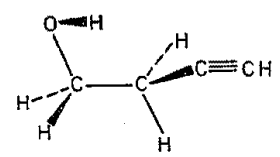

$\mathrm{C}_{4} \mathrm{H}_{6} \mathrm{O}$

$\mathrm{C}_{3}$

Observed molecular conformation was gauche, with evidence for an intramolecular hydrogen bond.

[1] L. D. Szalanski and R. G. Ford, J. Mol. Spcctrosc. 54, 148 (1975).

J. Phys. Chem. Ref. Data, Vol, 8, No. 3, 1979

\section{Cyclobutanone}

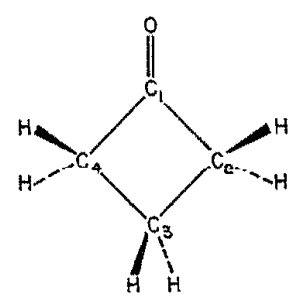

\begin{tabular}{|c|c|c|c|}
\hline Bond & Substitution & Angle & Substitution \\
\hline $\mathrm{C}_{1} \mathrm{C}_{2}$ & $1.529 \mathrm{~B}$ & $\mathrm{C}_{2} \mathrm{C}_{1} \mathrm{C}_{4}$ & $93.0 \mathrm{~B}$ \\
\hline $\mathrm{C}_{2} \mathrm{C}_{3}$ & $1.556 \mathrm{~B}$ & $\mathrm{C}_{1} \mathrm{C}_{2} \mathrm{C}_{3}$ & $88.1 \mathrm{~B}$ \\
\hline $\mathrm{CO}$ & $1.204 \mathrm{C}$ & $\mathrm{C}_{2} \mathrm{C}_{3} \mathrm{C}_{4}$ & $90.8 \mathrm{~B}$ \\
\hline $\mathrm{C}_{2} \mathrm{H}$ & $1.099 \mathrm{~B}$ & $\mathrm{HC}_{2} \mathrm{H}$ & $109.2 \mathrm{C}$ \\
\hline
\end{tabular}

The HCatt group is tilted toward the carbonyl group by $4.6^{\circ}$ (C).

[1] W. M. Stigliani and V. W. Laurie, J. Mol. Spectrosc. 62, 85 (1976).

2] L. H. Scharpen and V. M. Laurie, J. Chem. Phys. 49, 221 (1968).

\section{Dimethylketene}

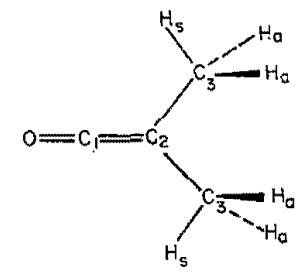

$\mathrm{C}_{4} \mathrm{H}_{6} \mathrm{O}$

\begin{tabular}{lll|lll}
\hline \hline \multirow{2}{*}{ Bond Substitution } & Effective & Angle & Substitution Effective \\
& & & $\mathrm{C}_{1} \mathrm{C}_{2} \mathrm{C}_{3}$ & & \\
$\mathrm{CII}_{3}$ & $1.088 \mathrm{C}$ & & $\mathrm{CCH}_{\mathrm{s}}$ & & $120.6 \mathrm{X}$ \\
$\mathrm{CH}_{\mathrm{s}}$ & $1.093 \mathrm{~B}$ & & $111.5 \mathrm{X}$ \\
$\mathrm{CO}$ & & 1.171 & $\mathrm{CCH}_{\mathrm{a}}$ & & $110.6 \mathrm{X}$ \\
$\mathrm{C}_{2} \mathrm{C}_{2}$ & & $1.300 \mathrm{X}$ & $\mathrm{H}_{\mathrm{a}} \mathrm{CH}_{\mathrm{s}}$ & $107.3 \mathrm{C}$ & \\
$\mathrm{C}_{2} \mathrm{C}_{3}$ & & $1.514 \mathrm{X}$ & $\mathrm{H}_{3} \mathrm{CH}_{\mathrm{a}}$ & $108.3 \mathrm{C}$ & \\
\hline
\end{tabular}

CO bond length was fixed at value reported for ketene.

[1] K. P. R. Nair, H. D. Rudolph, and H. Dreizler, J. Mol. Spectrose. 48, 571 (1973).

\section{3,4-Epoxy-1-butene}

$\mathrm{C}_{4} \mathrm{H}_{6} \mathrm{O}$

$$
\mathrm{H}_{2} \mathrm{C}=\mathrm{CH}-\mathrm{CH}-\mathrm{O}-\mathrm{CH}_{2}
$$

The molecule was shown to exist in the trans conformation relative to the CC single bond.

[1] T. Ikeda, K. V. I. N. Sastry and R. F. Curl, Jr., J. Mol. Spectrose. 56, $411(1975)$.

$\mathrm{Cov}_{28}$ 


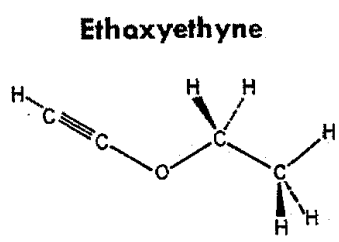

$\mathrm{C}_{4} \mathrm{H}_{6} \mathrm{O}$

The spectrum of the anti conformation, shown above, js consistent with a planar heavy-atom skeleton. A less stable gauche form, having a dihedral angle about the $\mathrm{CO}$ bond of $108^{\circ}(\mathrm{X})$, was also observed.

[1] A. Bjorseth, J. Mol. Struct, 20, 61 (1974).

\section{3-Methoxy-1-propyne (Methylpropargyl ether)}

$\mathrm{C}_{4} \mathrm{H}_{6} \mathrm{O}$

$$
\mathrm{CH}_{3}-\mathrm{O}-\mathrm{CH}_{2}-\mathrm{C} \equiv \mathrm{CH}
$$

$\mathrm{C}_{1}$

The observed stable molecular conformation was gauche with -peppect to the $\mathrm{C}$ hond, the repnrted dihedral angle being $68^{\circ} \cdot\left(0^{\circ}\right.$ = syn conformation).

[1] K. M. Marstokk and H. Mollendal, J. Mol. Struct. 32, 191 (1976).

\section{3-Methyleneoxetane}

$\mathrm{C}_{4} \mathrm{H}_{6} \mathrm{O}$

$$
\mathrm{H}_{2} \mathrm{C}=\mathrm{C}-\mathrm{CH}_{2}-\mathrm{O}-\mathrm{CH}_{3}
$$

$\mathrm{C}_{2 *}$

Analysis of ground and excited states of the ring-puckering vibration indicated a planar heavy-atom equilibrium structure.

[1] J. S. Gibson and D. 0. Harris, J. Chem. Phys. 52, 5234 (1970).

\section{trans-2-Methyl-2-propenal}

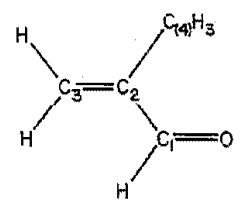

$\mathrm{C}_{4} \mathrm{H}_{6} \mathrm{O}$

\begin{tabular}{|c|c|}
\hline Angle & Effective \\
\hline $\begin{array}{l}\mathrm{C}_{3} \mathrm{C}_{2} \mathrm{C}_{4} \\
\mathrm{C}_{1} \mathrm{C}_{2} \mathrm{C}_{3}\end{array}$ & $\begin{array}{l}123.4 \mathrm{X} \\
116.4 \mathrm{X}\end{array}$ \\
\hline
\end{tabular}

$\mathrm{C}_{\mathrm{u}}$

Assumed values used for all other structural parameters.

[1] M. Suzuki and K. Kozima, J. Mol. Spectrosc. 38, 314 (1971).

\section{Methyl vinyl ketone.}

$\mathrm{C}_{4} \mathrm{H}_{6} \mathrm{O}$

$\mathrm{H}_{3} \mathrm{C}-\mathrm{CO}-\mathrm{CH}=\mathrm{CH}_{2}$

$\mathrm{C}_{\mathrm{s}}$

Observed conformation is that with the carbonyl trans to the vinyl group.

[1] P. D. Foster, V. M. Rao and R. F. Curl, Jr., J. Chem. Phys, 43,1064 (1965).

\section{Oxaspiro[2.2]pentane}

\begin{tabular}{|c|c|c|c|}
\hline Bond & Substitution & Angle & Substitution \\
\hline $\mathrm{C}_{2} \mathrm{O}$ & $1.460 \mathrm{~B}$ & $\mathrm{HC}_{2} \mathrm{H}$ & $117.0 \mathrm{~B}$ \\
\hline $\mathrm{C}_{2} \mathrm{C}_{4}$ & $1,550 \mathrm{~B}$ & $\mathrm{HC}_{3} \mathrm{H}$ & $115.0 \mathrm{C}$ \\
\hline $\mathrm{C}_{3} \mathrm{C}_{5}$ & $1.470 \mathrm{C}$ & $\mathrm{C}_{2} \mathrm{OC}_{65}$ & $60.4 B$ \\
\hline $\mathrm{C}_{5} \mathrm{O}$ & $1.416 \mathrm{C}$ & $\mathrm{C}_{2} \mathrm{C}_{5} \mathrm{O}$ & $61.3 \mathrm{C}$ \\
\hline $\mathrm{C}_{2} \mathrm{C}_{5}$ & $1.447 \mathrm{C}$ & $\mathrm{C}_{3} \mathrm{C}_{5} \mathrm{C}_{4}$ & $63.6 \mathrm{C}$ \\
\hline $\mathrm{C}_{2} \mathrm{H}$ & $1.086 \mathrm{~B}$ & $\mathrm{C}_{5} \mathrm{C}_{3} \mathrm{C}_{4}$ & $58.2 \mathrm{C}$ \\
\hline $\mathrm{C}_{3} \mathrm{H}_{\mathrm{b}}$ & $1.080 \mathrm{C}$ & $\mathrm{HC}_{2} \mathrm{C}_{5}$ & $119.5 \mathrm{~B}$ \\
\hline \multirow[t]{5}{*}{$\mathrm{C}_{3} \mathrm{H}_{\mathrm{c}}$} & $1.075 \mathrm{C}$ & $\mathrm{HC}_{2} \mathrm{O}$ & $114.1 \mathrm{~B}$ \\
\hline & & $\mathrm{H}_{b} \mathrm{C}_{3} \mathrm{H}_{5}$ & $118.9 \mathrm{C}$ \\
\hline & & $\mathrm{H}_{3} \mathrm{C}_{3} \mathrm{C}_{4}$ & $116.9 \mathrm{~B}$ \\
\hline & & $\mathrm{H}_{6} \mathrm{C}_{3} \mathrm{C}_{5}$ & $118.0 \mathrm{C}$ \\
\hline & & $\mathrm{H}_{8} \mathrm{C}_{3} \mathrm{C}_{4}$ & $116.9 \mathrm{~B}$ \\
\hline
\end{tabular}

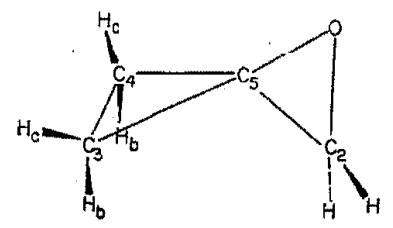

[1] W. D. Slafer, A. D. English, D. O. Harris, D. F. Shellhamer, M. J. Meshishnek and D. H. Aue, J. Am. Chem. Soc. 97, $6638(1975)$.

\section{2,3-Dihydro-p-dioxin \\ (1,4-Dioxene)}

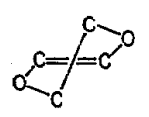

$\mathrm{C}_{4} \mathrm{H}_{6} \mathrm{O}_{2}$

The twist (half-chair) conformation was reliably established.

[1] J. A. Wells and T. B. Malloy, Ir., J. Chem. Phys. 60, 2132 (1974). 


\section{Ethylthicethyne}

$\mathrm{C}_{4} \mathrm{H}_{6} \mathrm{~S}$

$\left(\mathrm{CH}_{3} \mathrm{CH}_{2}\right) \mathrm{S}(\mathrm{C} \equiv \mathrm{CH})$

The observed spectrum was consistent only with the methyl group occupying a gauche conformation, $119^{\circ}(\mathrm{X})$ from the anti position.

[1] A. Bjorseth, J. Mol. Struet. 23, 1 (1974).

\section{Bromocyclobutane}

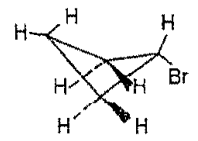

$\mathrm{C}_{4} \mathrm{H}_{7} \mathrm{Br}$

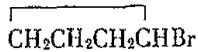

Ring is puckered with a dihedral angle of approximately $29^{\circ}$. Only the equatorial form was observed.

[1] W. G. Rothschild and B. P. Dailey, J. Chem. Phys. 36, 2931 (1962).

Chlorocyclobutane

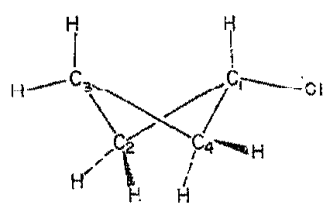

$\mathrm{C}_{4} \mathrm{H}_{7} \mathrm{Cl}$

\begin{tabular}{cc|cc}
\hline \hline Bond & Effective & Angle & Fffective \\
\hline $\mathrm{C}_{1} \mathrm{Cl}$ & $1.775 \mathrm{D}$ & $\mathrm{HCCl}$ & $114 \mathrm{D}$ \\
$\mathrm{C}_{1} \mathrm{C}_{2}$ & $1.525 \mathrm{D}$ & $\mathrm{C}_{2} \mathrm{C}_{2} \mathrm{C}_{4}$ & $91 \mathrm{D}$ \\
$\mathrm{C}_{2} \mathrm{C}_{3}$ & $1.550 \mathrm{D}$ & $\mathrm{HC}_{2} \mathrm{H}$ & $112 \mathrm{D}$ \\
$\mathrm{C}_{1} \mathrm{H}$ & $1.10 \mathrm{D}$ & $\mathrm{HC}_{3} \mathrm{H}$ & $110 \mathrm{D}$ \\
$\mathrm{C}_{2} \mathrm{H}$ & $1.09 \mathrm{D}$ & $\mathrm{HC}_{2} \mathrm{C}_{1}$ & $114 \mathrm{D}$ \\
$\mathrm{C}_{3} \mathrm{H}$ & $1.10 \mathrm{D}$ & $\begin{array}{c}\text { ring } \\
\text { rihedral }\end{array}$ & $20 \mathrm{D}$ \\
$\mathrm{CCl}$ with & \\
$\mathrm{C}_{2} \mathrm{C}_{2} \mathrm{C}_{4}$ plane & $135 \mathrm{D}$ \\
& &
\end{tabular}

$\mathrm{CH}_{2}$ groups were assumed to bisect ring angles. Only equatorial Cl observed.

[1] H. Kim and W. D. Gwinn, J. Chem. Phys. 44, 865 (1966).
cis-1-Butene

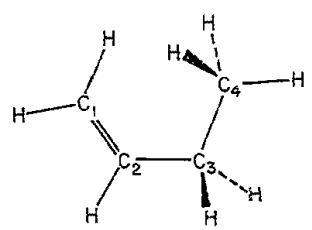

$\mathrm{C}_{4} \mathrm{H}_{8}$

\begin{tabular}{cc|cc}
\hline \hline Bond & Effective & Angle & Effective \\
\hline & $1.336 \mathrm{D}$ & & \\
$\mathrm{C}_{2} \mathrm{C}_{2}$ & $1.507 \mathrm{D}$ & $\mathrm{C}_{3} \mathrm{C}_{2} \mathrm{C}_{4}$ & $126.7 \mathrm{D}$ \\
$\mathrm{C}_{2} \mathrm{C}_{3}$ & $\mathrm{C}_{2} \mathrm{C}_{3} \mathrm{C}_{4}$ & $114.8 \mathrm{D}$ \\
$\mathrm{C}_{3} \mathrm{C}_{4}$ & $1.536 \mathrm{D}$ & $\mathrm{HC}_{2} \mathrm{C}_{3}$ & $115.1 \mathrm{D}$ \\
& & $\mathrm{HC}_{4} \mathrm{C}_{3}$ & $110.4 \mathrm{D}$ \\
& & $\mathrm{HC}_{4} \mathrm{H}$ & $105.2 \mathrm{D}$ \\
\hline
\end{tabular}

$\mathrm{CH}$ bond lengths were assumed. $\mathrm{HC}_{3} \mathrm{H}$ was assumed to bisect $\mathrm{C}_{2} \mathrm{C}_{3} \mathrm{C}_{4}$. The shew form was also observed. See the following.

[J.] S. Kondo, E. Hirota, Y. Morino, J. Mol. Spectrusti. 28, 471 (1968).

\section{skew-1-Butene}

\begin{tabular}{llll}
$\mathrm{C}_{4} \mathrm{H}_{8}$ & $\mathrm{C}^{1} \mathrm{H}_{2}=\mathrm{C}^{2} \mathrm{HC}^{2} \mathrm{H}_{2} \mathrm{C}^{4} \mathrm{H}_{3}$ & $\mathrm{C}_{1}$ \\
\hline Rand & Efrective & Angio & Effoctive \\
\hline & & & \\
$\mathrm{C}^{1} \mathrm{C}^{2}$ & $1.342 \mathrm{D}$ & $\mathrm{C}^{1} \mathrm{C}^{2} \mathrm{C}^{8}$ & $125.4 \mathrm{C}$ \\
$\mathrm{C}^{2} \mathrm{C}^{3}$ & $1.493 \mathrm{D}$ & $\mathrm{C}^{2} \mathrm{C}^{3} \mathrm{C}^{4}$ & $112.1 \mathrm{C}$ \\
$\mathrm{C}^{2} \mathrm{C}^{4}$ & $1.536 \mathrm{D}$ & dihedral $^{\mathrm{s}}$ & $119.9 \mathrm{C}$ \\
& & $\mathrm{HC}^{2} \mathrm{C}^{8}$ & $117.1 \mathrm{E}$ \\
& & $\mathrm{HC}^{4} \mathrm{C}^{3}$ & $110.3 \mathrm{D}$ \\
& & $\mathrm{HC}^{3} \mathrm{H}$ & $105.7 \mathrm{D}$ \\
\hline
\end{tabular}

$\mathrm{CH}$ bond lengths were assumed. The cis form was also observed. See the preceding.

${ }^{a}$ Angle between $C_{8} C_{4} C_{* 3}$ and $C_{8} C_{0} C_{4}$ plares.

[1] S. Kondo, E. Hirota, and Y. Morino, J. Mol. Spectrosc. 28, 471 (1968).

\section{cis-2-Butene}

\begin{tabular}{cccc}
$\mathrm{C}_{4} \mathrm{H}_{8}$ & \multicolumn{2}{c}{$\mathrm{H}_{3} \mathrm{CCH}=\mathrm{CHCH}_{3}$} & $\mathrm{CH}_{28}$ \\
\hline Bond & Effective & Angle & Effective \\
\hline $\mathrm{CC}$ & $1.497 \mathrm{D}$ & $\mathrm{CCC}$ & $126.7 \mathrm{D}$ \\
\hline
\end{tabular}

A number of structural parameters were assumed.

[1] S. Koudo, Y. Sakurai, E. Hirota, and Y. Morino, J. Mol. Spectrose. 34,231 (1970) 


\begin{tabular}{|c|c|c|c|}
\hline \multirow[b]{2}{*}{$\mathrm{C}_{4} \mathrm{H}_{8}$} & \multicolumn{2}{|c|}{ Cyclobutane } & \multirow[b]{2}{*}{$D_{2 C}$} \\
\hline & & $\mathrm{H}_{2} \mathrm{CH}_{2}$ & \\
\hline Bond & Effective & Angle & Effective \\
\hline $\mathrm{CC}$ & $1.558 \mathrm{C}$ & ring dihedral & $35 \mathrm{E}$ \\
\hline
\end{tabular}

[1] K. C. Lord and B. P. Stoicheff, Cani. J. Phys. 40, 725 (1962).

[2] J. M. R. Stone and I. M. Mills, Mol. Phys. 18, 63l (1970).

[3] F. A. Miller and R. J. Capwell, Spectrochim. Acta 27 A, 947 (1971).

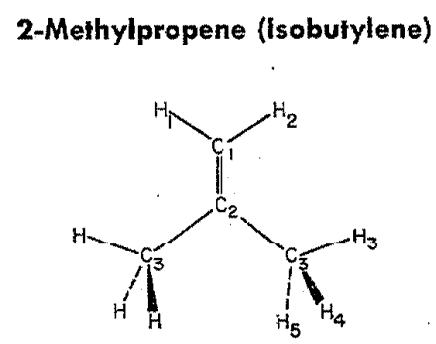

\begin{tabular}{cc|cc}
\multicolumn{1}{c}{} & & \\
\hline \hline Bond & Substitution & Angle & Substitution \\
\hline & & & \\
\hline $\mathrm{C}_{2} \mathrm{C}_{2}$ & $1.330 \mathrm{D}$ & $\mathrm{C}_{1} \mathrm{C}_{2} \mathrm{C}_{3}$ & $115.3 \mathrm{D}$ \\
$\mathrm{C}_{2} \mathrm{C}_{3}$ & $1.507 \mathrm{C}$ & $\mathrm{H}_{1} \mathrm{C}_{1} \mathrm{H}_{2}$ & $118.5 \mathrm{~B}$ \\
$\mathrm{C}_{2} \mathrm{H}_{1}$ & $1.088 \mathrm{~B}$ & $\mathrm{C}_{2} \mathrm{C}_{3} \mathrm{H}_{3}$ & $112.9 \mathrm{E}$ \\
$\mathrm{C}_{8} \mathrm{H}_{3}$ & $1.072 \mathrm{E}$ & $\mathrm{C}_{2} \mathrm{C}_{3} \mathrm{H}_{4}$ & $110.7 \mathrm{C}$ \\
$\mathrm{C}_{3} \mathrm{H}_{4}$ & $1.095 \mathrm{C}$ & $\mathrm{H}_{4} \mathrm{C}_{8} \mathrm{H}_{4}$ & $106.0 \mathrm{C}$ \\
& & & \\
\hline
\end{tabular}

[1] L. H. Scharpen and V. W. Laurie, J. Chem. Phys. 39, 1732 (1963).

[2] V. W. Laurie, J. Chem. Phys. 34, 1516 (1961).

Tefrahydroselenophene

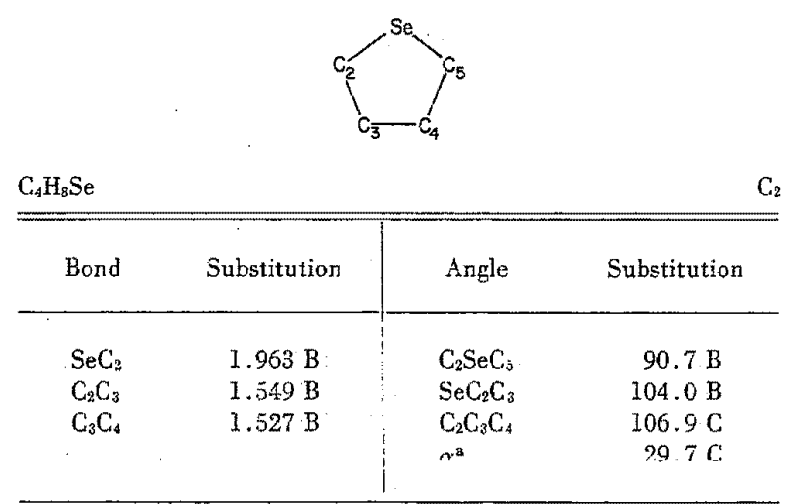

- Twist angle formed by intersection of $\mathrm{C}_{5} \mathrm{SeC}_{2}$ plane and $\mathrm{C}_{4} \mathrm{SeC}_{8}$ plane.

[1] A. H. Mamleev, N. M. Pozdeev and N. N. Magdesieva, J. Mol. Struct. 33, 211 (1976).
2-Chloro-2-methylpropane

(Tertiary butyl chloride)

\begin{tabular}{|c|c|c|c|c|c|}
\hline${ }_{4} \mathrm{H}_{9} \mathrm{Cl}$ & \multicolumn{4}{|c|}{$\left(\mathrm{CH}_{3}\right)_{3} \mathrm{CCl}$} & \multirow[b]{2}{*}{ Average } \\
\hline Bond & $\begin{array}{l}\text { Substitu- } \\
\text { tion }\end{array}$ & Average & Angle & $\begin{array}{l}\text { Substitu- } \\
\text { tion }\end{array}$ & \\
\hline $\begin{array}{l}\mathrm{CCl} \\
\mathrm{CC}\end{array}$ & $\begin{array}{l}1.303 \mathrm{~B} \\
1.530 \mathrm{~B}\end{array}$ & $\begin{array}{l}1.831 \mathrm{D} \\
1.525 \mathrm{~B}\end{array}$ & $\mathrm{CCCl}$ & $108.0 \mathrm{~B}$ & $107.0 \mathrm{C}$ \\
\hline Bond & \multicolumn{2}{|c|}{ Effective } & Angle & \multicolumn{2}{|r|}{ Effective } \\
\hline $\mathrm{CCl}$ & \multicolumn{2}{|c|}{$1.828 \mathrm{~B}$} & $\mathrm{CCO}$ & \multicolumn{2}{|r|}{$111.7 \mathrm{D}$} \\
\hline $\mathrm{CC}$ & \multirow{2}{*}{\multicolumn{2}{|c|}{$\begin{array}{l}1.528 \mathrm{~B} \\
1.097 \mathrm{~B}\end{array}$}} & $\mathrm{HCH}$ & \multirow{2}{*}{\multicolumn{2}{|c|}{$109.4 \mathrm{~B}$}} \\
\hline $\mathrm{CH}$ & & & & & \\
\hline
\end{tabular}

[1] D. R. Lide and M. Jen, J. Chem. Phys. 38, 1504 (1963).

[2] R. L. Hilderbrandt and J. D. Wieser, J. Chem, Phys. 56, 1143 (1972).

[3] W. Braun, H. Günther, H. Umbrecht and W. Zeil, Z. Physik. Chem. 93, 247 (1974).

\begin{tabular}{|c|c|c|c|}
\hline \multirow{3}{*}{$\frac{\mathrm{C}_{4} \mathrm{H}_{4} \mathrm{~F}}{\text { Bond }}$} & \multicolumn{3}{|c|}{$\begin{array}{l}\text { 2-Fluoro-2-methylpropane } \\
\text { (Tertiary Butyl Fluoride) }\end{array}$} \\
\hline & \multicolumn{2}{|c|}{$\left(\mathrm{CH}_{3}\right)_{3} \mathrm{CF}$} & $C_{38}$ \\
\hline & Effective & Angle & Effective \\
\hline $\mathrm{CF}$ & $1.43 \quad \mathrm{D}$ & $\mathrm{CCC}$ & $112.7 \mathrm{C}$ \\
\hline $\mathrm{CC}$ & $1.516 \mathrm{C}$ & $\mathrm{HCH}$ & $107.9 \mathrm{D}$ \\
\hline
\end{tabular}

$\mathrm{CH}$ distance was fixed at $1.090 \AA$.

[1] D. R. Lide and D. E. Mann, J.' Chem. Phys. 29, 9l4 (1958).

\section{Trimethylgermanecarbonitrile (Trimethylcyanogermane)}

\begin{tabular}{lcc|cc}
$\mathrm{C}_{4} \mathrm{H}_{9} \mathrm{GeN}$ & & $\left(\mathrm{CH}_{3}\right)_{3} \mathrm{GeCN}$ & $\mathrm{C}_{3 v}$ \\
\hline Bond & Substitution & Effective & Angle & Effective \\
& & & & \\
$\mathrm{CN}$ & $1.115 \mathrm{~A}$ & & $\mathrm{CGeC}(\mathrm{N})$ & $106.2 \mathrm{X}$ \\
$\mathrm{GeC}(\mathrm{N})$ & $1.947 \mathrm{C}$ & & $\mathrm{HCGe}$ & $111.0^{3}$ \\
$\mathrm{GeC}\left(\mathrm{H}_{3}\right)$ & & $1.930 \mathrm{X}$ & & \\
$\mathrm{CH}$ & & $1.095^{\circ}$ & & \\
\hline
\end{tabular}

Assumed values.

[1] J. R. Durig, Y. S. Li and J. B. Turner, Inorg. Chem. 13, 1495 (1974). 


\section{2-lodo-2-methylpropane \\ (Tertiary butyl iodide)}

\begin{tabular}{cc|cc}
$\mathrm{C}_{4} \mathrm{H}_{9} \mathrm{I}$ & $\left(\mathrm{CH}_{3}\right)_{3} \mathrm{CI}$ & $\mathrm{C}_{3 \mathrm{v}}$ \\
\hline Bond & Effective & Angle & Effective \\
\hline $\mathrm{CI}$ & $2.190 \mathrm{C}$ & $\mathrm{CCC}$ & $111.0 \mathrm{~A}$ \\
$\mathrm{CC}$ & $1.527 \mathrm{~B}$ & & \\
\hline
\end{tabular}

The parameters $\mathrm{CC}, \mathrm{CH}$ and $\mathrm{CCC}$ were assumed to obtain $\mathrm{CI}$. [1] J. Q. Willians and W. Gordy, J. Chem. Phys. 18, 994, (1950). [2] W. Winkle and H. Hartmann, Z. Naturforsch. 25a, 840 (1970).

\section{Trimethylsilyl isocyanate}

$\mathrm{C}_{4} \mathrm{H}_{9} \mathrm{NOSi}$

$$
\left(\mathrm{CH}_{3}\right)_{3} \mathrm{SiNCO}
$$

$\mathrm{C}_{3 \mathrm{v}}$

The spectral evidence is consistent with a linear or very nearly linear $\mathrm{Si}-\mathrm{N}=\mathrm{C}=\mathrm{O}$ group.

[1] A. J. Careless, M. C. Green and H. W. Kroto, Chem. Phys Lett. 16, 414 (1972).

\section{Trimethylsilyleyanide}

\section{$\mathrm{C}_{4} \mathrm{H}_{2} \mathrm{NSi}$}

$\left(\mathrm{CH}_{3}\right)_{3} \mathrm{SiCN}$

$\mathrm{C}_{3 \mathrm{v}}$

The conventionally prepared sample of this compound was found to contain (based upon symmetric rotor spectra for each isomer) approximately $5 \%$ of the isocyanide (-SiNC) isomer.

[1] J. R. Durig, W. O. George, Y. S. Li and R. O. Carter, J. Mol. Struct. 16, 47 (1973)

\section{2-Mełhyl propane \\ (Isobutane)}

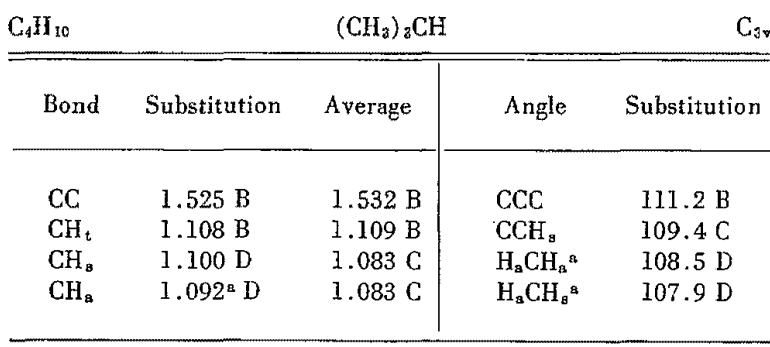

Average structure parameters are $r_{\mathrm{z}}$ values from combined microwave-electron diffraction data. $\mathrm{H}_{2}$ refers to tertiary hydrogen, and $\mathrm{H}_{\mathrm{B}}$ and $\mathrm{H}_{\mathrm{a}}$ refer to the in-plane and out-of-plane atoms, respectively.

shese parameters utilized assumption that methyl hydrogeno form an equilateral triangle.

[1] D. R. Lide, J. Chem. Phys. 33, 1519 (1960).

[2] R. L. Hilderbrandt and J. D. Wieser, J. Mol. Struct. 15, 27 (1973).

\section{Germacyclopentare}

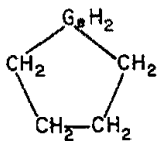

$\mathrm{C}_{4} \mathrm{H}_{10} \mathrm{Ge}$

$\mathrm{C}_{2}$

\begin{tabular}{lr}
\hline Angle $^{\circledR}$ & Effective \\
\hline CGeC & $98^{\circ} \mathrm{X}$ \\
CCGe & $106^{\circ} \mathrm{X}$ \\
CCC & $115^{\circ} \mathrm{X}$ \\
Twist & $18^{\circ} \mathrm{X}$
\end{tabular}

Determined by fitting moments of inertia along with the as sumptions $\mathrm{CGe}=1.95 \mathrm{~A}, \mathrm{CC}=1.53 \mathrm{~A}, \mathrm{CH}=1.09 \mathrm{~A}, \mathrm{GeH}=$ $1.53 \mathrm{~A}, \mathrm{HCH}=109^{\circ}, \mathrm{HGeH}=111^{\circ}$.

[1] E. C. Thomas and V. W. Laurie, J. Chem. Phys. 51, 4327 (1969).

\section{Diethyl ether}

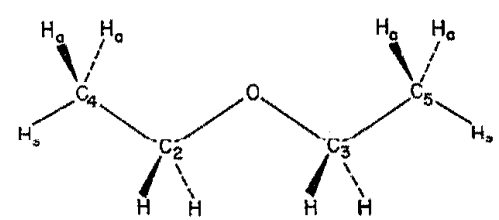

$\mathrm{C}_{4} \mathrm{H}_{10} \mathrm{O}$

$\mathrm{C}_{2 \mathrm{~V}}$

\begin{tabular}{|c|c|c|c|c|c|}
\hline Bunel & $\begin{array}{l}\text { Substitu- } \\
\text { tiun }\end{array}$ & Emetive & Angle & $\begin{array}{l}\text { Substitu- } \\
\text { tion }\end{array}$ & Effective \\
\hline $\mathrm{CO}$ & $1.408 \mathrm{~B}$ & & $\mathrm{COC}$ & $112.6 \mathrm{~B}$ & \\
\hline $\mathrm{cc}$ & $1.516 \mathrm{~B}$ & & OCC & $108.6 \mathrm{~B}$ & \\
\hline $\mathrm{C}_{2} \mathrm{H}$ & $1.100 \mathrm{~B}$ & & $\mathrm{C}_{4} \mathrm{C}_{2} \mathrm{H}$ & I10.4 B & \\
\hline $\mathrm{C}_{4} \mathrm{H}_{8}$ & $1.090 \mathrm{~B}$ & & & & \\
\hline \multirow[t]{4}{*}{$\mathrm{C}_{4} \mathrm{H}_{\mathrm{B}}$} & & $1.090 \mathrm{X}$ & $\mathrm{OC}_{2} \mathrm{H}$ & $109.9 \mathrm{~B}$ & \\
\hline & & & $\mathrm{C}_{2} \mathrm{C}_{4} \mathrm{H}_{8}$ & $110.2 \mathrm{~B}$ & \\
\hline & & & $\mathrm{C}_{2} \mathrm{C}_{4} \mathrm{H}_{8}$ & & $110.6 \mathrm{D}$ \\
\hline & & & $\begin{array}{l}\mathrm{M}_{\mathrm{B}} \mathrm{C}_{4} \mathrm{n}_{\mathrm{B}} \\
\mathrm{H}_{8} \mathrm{C}_{4} \mathrm{H}_{\mathrm{B}}\end{array}$ & $108.1 \mathrm{C}$ & \\
\hline
\end{tabular}

The $b$ coordinate of $\mathrm{H}_{a}$ was computed under the assumption that $\mathrm{C}_{4} \mathrm{H}_{\mathrm{B}}=\mathrm{C}_{4} \mathrm{H}_{\mathrm{B}}$. The coordinates of the methylene $\mathrm{H}$ atoms were recomputed from the reported rotational constants.

[1] M. Hayashi and K. Kuwada, Bull. Chem. Soc. Japan 47, 3006, 1974.

[2] M. Hayashi and K. Kuwada, Bull. Chem. Soc. Japan 44, 299 (1971). 


\section{Methylpropyl ether}

$\mathrm{C}_{4} \mathrm{H}_{10} \mathrm{O}$

$\left(\mathrm{CH}_{3}\right) \mathrm{O}\left(\mathrm{C}_{3} \mathrm{H}_{7}\right)$

$\mathrm{C}_{\mathrm{B}}$

The observed spectrum is consistent only with the trans-trans conformation.

[1] M. Hayashi, M. Imachi, J. Nakagawa and A. Ozaki, Chem. Lett. (Japan) 1977, 41 (1977).

\section{$C_{5}-C_{11}$ Molecules}

Cyanobutadiyne

(Cyanodiacetylene)

\begin{tabular}{cc}
$\mathrm{C}_{5} \mathrm{HN} \quad \mathrm{H}-\mathrm{C}^{1} \equiv \mathrm{C}^{2}-\mathrm{C}^{3}=\mathrm{C}^{4}-\mathrm{C}^{5}=\mathrm{N}$ & $\mathrm{C}_{\infty \rightarrow \infty}$ \\
\hline \hline Bond & Substitution \\
\hline $\mathrm{CH}$ & $1.057 \mathrm{~B}$ \\
$\mathrm{C}^{1} \mathrm{C}^{2}$ & $1.209 \mathrm{~A}$ \\
$\mathrm{C}^{2} \mathrm{C}^{5}$ & $1.362 \mathrm{~B}$ \\
$\mathrm{C}^{3} \mathrm{C}^{4}$ & $1.222 \mathrm{~B}$ \\
$\mathrm{C}^{4} \mathrm{C}^{5}$ & $1.364 \mathrm{~B}$ \\
$\mathrm{C}^{5} \mathrm{~N}$ & $1.261 \mathrm{~A}$
\end{tabular}

[1] A. J. Alexander, H. W. Kroto and D. R. M. Walter, J. Mol. Spectrosc. 62, 175 (1976).

\section{2,6-Difluoropyridine}

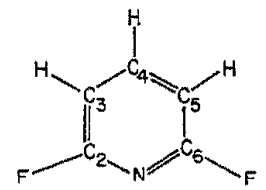

\begin{tabular}{lcc|ccc}
$\mathrm{C}_{5} \mathrm{H}_{3} \mathrm{~F}_{2} \mathrm{~N}$ & & & $\mathrm{C}_{2 \mathrm{v}}$ \\
\hline \hline Bond & $\begin{array}{c}\text { Substitu- } \\
\text { tion }\end{array}$ & Effective & Angle & $\begin{array}{c}\text { Substitu- } \\
\text { tion }\end{array}$ & Effective \\
\hline $\mathrm{NC}_{2}$ & $1.317 \mathrm{~B}$ & & $\mathrm{C}_{3} \mathrm{C}_{4} \mathrm{C}_{5}$ & $119.8 \mathrm{~A}$ & \\
$\mathrm{C}_{2} \mathrm{C}_{3}$ & $1.377 \mathrm{C}$ & & $\mathrm{C}_{2} \mathrm{C}_{3} \mathrm{C}_{4}$ & $116.1 \mathrm{~A}$ & \\
$\mathrm{C}_{3} \mathrm{C}_{4}$ & $1.394 \mathrm{~A}$ & & $\mathrm{NC}_{2} \mathrm{C}_{3}$ & $126.5 \mathrm{~B}$ & \\
$\mathrm{C}_{3} \mathrm{H}$ & $1.080 \mathrm{~B}$ & & $\mathrm{C}_{2} \mathrm{NC}_{6}$ & $115.0 \mathrm{~B}$ & \\
$\mathrm{C}_{4} \mathrm{HI}$ & $1.082 \mathrm{~B}$ & & $\mathrm{C}_{3} \mathrm{C}_{4} \mathrm{H}$ & $120.1 \mathrm{~B}$ & \\
$\mathrm{C}_{2} \mathrm{~F}$ & & $1.347 \mathrm{~B}$ & $\mathrm{C}_{2} \mathrm{C}_{3} \mathrm{H}$ & $120.8 \mathrm{~B}$ & \\
& & & $\mathrm{C}_{3} \mathrm{C}_{2} \mathrm{~F}$ & & $118.8 \mathrm{~B}$ \\
& & & & & \\
& & & & &
\end{tabular}

[1] 0. L. Stiefvater, Z. Naturforsch. 30a, 1765 (1975).
4H-Thiapyran-4-thione<smiles>S=C1C=CCSC1</smiles>

$\mathrm{C}_{5} \mathrm{H}_{4} \mathrm{~S}_{2}$

\begin{tabular}{ll|ll}
\hline \hline Bond & Substitution & Angle & Substitution \\
\cline { 3 - 4 } & & & \\
\cline { 3 - 3 } $\mathrm{C}_{1} \mathrm{~S}$ & $1.671 \mathrm{~B}$ & $\mathrm{C}_{2} \mathrm{C}_{1} \mathrm{C}_{2}$ & $117.5 \mathrm{D}$ \\
$\mathrm{C}_{3} \mathrm{~S}$ & $1.759 \mathrm{~A}$ & $\mathrm{C}_{1} \mathrm{C}_{2} \mathrm{C}_{3}$ & $128.1 \mathrm{D}$ \\
$\mathrm{C}_{1} \mathrm{C}_{2}$ & $1.406^{\mathrm{a}} \mathrm{D}$ & $\mathrm{C}_{2} \mathrm{C}_{3} \mathrm{~S}$ & $122.4 \mathrm{C}$ \\
$\mathrm{C}_{2} \mathrm{C}_{3}$ & $1.342^{\mathrm{a}} \mathrm{D}$ & $\mathrm{C}_{3} \mathrm{SC}_{3}$ & $101.4 \mathrm{~A}$ \\
\hline
\end{tabular}

* These parameters required that hydrogen positions be fixed by assumption.

[1] M. J. Corkill, A. P. Cox and I. C. Ewart, J. C. S. Chem. Comm. $1976,546(1976)$.

\section{Cyclopentadienylberyllium chloride}

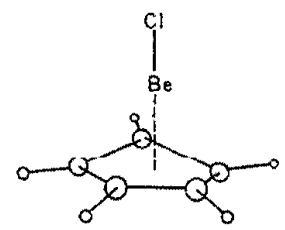

$\mathrm{C}_{5} \mathrm{H}_{5} \mathrm{BeCl}$

$\mathrm{C}_{5 \mathrm{v}}$

\begin{tabular}{lll}
\hline Bond & Substitituon & Effective \\
\hline $\mathrm{CC}$ & $1.424 \mathrm{~B}$ & $1.424 \mathrm{~B}$ \\
$\mathrm{BeCl}$ & $1.81 \mathrm{E}$ & $1.839 \mathrm{C}$ \\
$\mathrm{h}^{\mathrm{a}}$ & $1.52 \mathrm{E}$ & $1.485 \mathrm{C}$ \\
$\mathrm{CH}$ & $1.09 \mathrm{E}$ & $1.090 \mathrm{D}$ \\
$\mathrm{Cl} \cdot \mathrm{C}$ & $3.546 \mathrm{~B}$ & $3.538 \mathrm{~B}$ \\
& & \\
\hline
\end{tabular}

Hydrogen atoms were assumed to be coplanar with the fivemembered ring. Also, to obtain $\mathrm{CH}$ substitution distance, the value $\mathrm{B}-\mathrm{C}=26.8 \mathrm{MHz}$ was assumed for the monodeuterated species.

a Distance of $\mathrm{Be}$ atom from plane of the five-membered ring.

[1] A. Bjorseth, D. A. Drew, K. M. Marstokk and H. Mollendal, J. Mol. Struct. 13, 233 (1972). 
Pyridine<smiles>c1ccccc1</smiles>

Pyridine N-oxide<smiles>[O-][n+]1ccccc1</smiles>

\begin{tabular}{cc|cc}
$\mathrm{C}_{5} \mathrm{H}_{5} \mathrm{~N}$ & & $\mathrm{C}_{2 v}$ \\
\hline \hline Bond & Substitution & Angle & Substitution \\
\hline & & & \\
\hline $\mathrm{NC}_{2}$ & $1.338 \mathrm{~A}$ & $\mathrm{C}_{2} \mathrm{NC}_{2}$ & $116.0 \mathrm{~A}$ \\
$\mathrm{C}_{2} \mathrm{C}_{3}$ & $1.394 \mathrm{~B}$ & $\mathrm{NC}_{2} \mathrm{C}_{3}$ & $123.8 \mathrm{~A}$ \\
$\mathrm{C}_{3} \mathrm{C}_{4}$ & $1.392 \mathrm{~A}$ & $\mathrm{C}_{2} \mathrm{C}_{3} \mathrm{C}_{4}$ & $118.5 \mathrm{~A}$ \\
$\mathrm{C}_{2} \mathrm{H}$ & $1.086 \mathrm{~B}$ & $\mathrm{C}_{3} \mathrm{C}_{4} \mathrm{C}_{5}$ & $118.4 \mathrm{~A}$ \\
$\mathrm{C}_{3} \mathrm{H}$ & $1.082 \mathrm{~B}$ & $\mathrm{NC}_{2} \mathrm{H}$ & $116.0 \mathrm{~B}$ \\
$\mathrm{C}_{4} \mathrm{H}$ & $1.081 \mathrm{~B}$ & $\mathrm{C}_{2} \mathrm{C}_{3} \mathrm{H}$ & $120.1 \mathrm{~B}$ \\
& & $\mathrm{C}_{3} \mathrm{C}_{4} \mathrm{H}$ & $120.8 \mathrm{~B}$ \\
& & & \\
\hline
\end{tabular}

[1] G. O. Sorensen, L. Moliler and N. Rash up Andersen, J. Mul. Struct. 20, 119 (1974).

[2] B. Bak, L. Hansen-:Nygaard and J. Rastrup-Andersen, J. Mol Spectrosc. 2, 361 (1958).

\section{a-Cyclopentadienylnitrosylnickel}

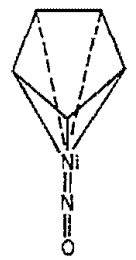

\begin{tabular}{lll}
\hline \hline Bond & Substitution & Effective \\
\hline $\mathrm{NO}$ & $1.165 \mathrm{~B}$ & \\
$\mathrm{NiN}$ & $1.626 \mathrm{C}$ & \\
$\mathrm{NiC}$ & & $2.11 \mathrm{C}$ \\
$\mathrm{CC}$ & & $1.43 \mathrm{C}$
\end{tabular}

No information regarding the planarity of the $\mathrm{C}_{5} \mathrm{H}_{5}$ ring system (i.e., coplanarity of $\mathrm{C}_{5}, \mathrm{H}_{5}$ planes) was obtained. The $\mathrm{CH}$ distances were assumed.

[1] A, P. Cox and A. H. Brittain, Trans. Faraday Soc. 66, 557 (1970).

\begin{tabular}{lcc|cc}
$\mathrm{C}_{5} \mathrm{H}_{3} \mathrm{NO}$ & & $\mathrm{C}_{2 \mathrm{v}}$ \\
\hline Bond & Substitution & Effective & Angle & Substitution \\
\hline & & & & \\
$\mathrm{NC}_{2}$ & $1.262 \mathrm{C}$ & & $\mathrm{CNC}$ & $119.8 \mathrm{C}$ \\
$\mathrm{C}_{2} \mathrm{C}_{3}$ & $1.389 \mathrm{C}$ & & $\mathrm{NCC}$ & $120.7 \mathrm{~B}$ \\
$\mathrm{C}_{3} \mathrm{C}_{4}$ & $1.395 \mathrm{~A}$ & & $\mathrm{C}_{2} \mathrm{C}_{3} \mathrm{C}_{4}$ & $120.6 \mathrm{~A}$ \\
$\mathrm{NO}$ & & $1.278 \mathrm{D}$ & $\mathrm{C}_{3} \mathrm{C}_{4} \mathrm{C}_{3}$ & $117.6 \mathrm{~A}$ \\
& & & & \\
\hline
\end{tabular}

Hydrogen parameters were assumed to obtain the NO distance. [1] 0. Snerling, C. J. Nielsen, L. Nygaard, E. J. Pedersen and G. 0 . Sorensen, J. Mol. Struc. 27, 205 (1975).

\section{cis-Pyrrole-2-carboxaldehyde}

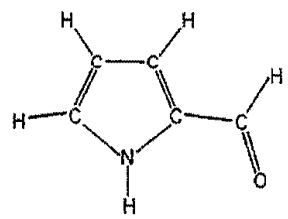

$\mathrm{C}_{5} \mathrm{H}_{5} \mathrm{NO}$

$\mathrm{C}_{s}$

The cis conformation was definitely established with a non-bonding $0 \cdots \mathrm{H}$ distance of $2.592(\mathrm{C})$.

[1] K. M. Marstokk and H. Mollendal, J. Mol. Struct. 23, 93 (1974).

\section{Cyclopentadienyl Thallium}

$\mathrm{C}_{5} \mathrm{H}_{5} \mathrm{Tl}$

$\mathrm{C}_{\tilde{5} \mathrm{v}}$

\begin{tabular}{cc}
\hline Bond & Effective \\
\hline & \\
CC & $1.43 \times$ \\
CTI & $2.705 \mathrm{X}$
\end{tabular}

$\mathrm{C}_{5} \mathrm{H}_{5}$ moiety was assumed to be coplanar, with $\mathrm{CH}=1.080 \AA$. [1] J. K. Tyler, A. P. Cox and J. Sheridan, Nature 183, 1182 (1959). 


\section{Cyclopenjadiene}

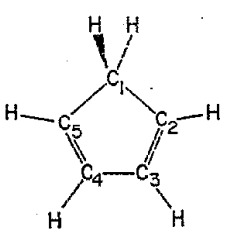

\begin{tabular}{cc|cc}
$\mathrm{C}_{5} \mathrm{H}_{6}$ & & $\mathrm{C}_{2 \mathrm{v}}$ \\
\hline Bond & Substitution & Angle & Substitution \\
\hline & & & \\
$\mathrm{C}_{1} \mathrm{C}_{2}$ & $1.506 \mathrm{~B}$ & $\mathrm{C}_{3} \mathrm{C}_{2} \mathrm{C}_{2}$ & $102.9 \mathrm{~B}$ \\
$\mathrm{C}_{2} \mathrm{C}_{3}$ & $1.344 \mathrm{~B}$ & $\mathrm{C}_{1} \mathrm{C}_{2} \mathrm{C}_{3}$ & $109.2 \mathrm{~B}$ \\
$\mathrm{C}_{3} \mathrm{C}_{4}$ & $1.468 \mathrm{~B}$ & $\mathrm{C}_{2} \mathrm{C}_{3} \mathrm{C}_{4}$ & $109.3 \mathrm{~B}$ \\
$\mathrm{C}_{1} \mathrm{H}$ & $1.099 \mathrm{~B}$ & $\mathrm{HC}_{1} \mathrm{H}$ & $106.3 \mathrm{~B}$ \\
$\mathrm{C}_{2} \mathrm{H}$ & $1.078 \mathrm{~B}$ & $\mathrm{HC}_{2} \mathrm{C}_{3}$ & $127.1 \mathrm{~B}$ \\
$\mathrm{C}_{3} \mathrm{H}$ & $1.080 \mathrm{~B}$ & $\mathrm{HC}_{3} \mathrm{C}_{2}$ & $126.0 \mathrm{~B}$ \\
& & &
\end{tabular}

[1] D. Damianni, L. Ferretti and E. Gallinella, Chem. Phys. Letz. $37,265(1976)$

[2] L. H. Scharpen and V. W. Laurie, J. Chem, Phys. 43, 2765 (1965).

\begin{tabular}{|c|c|c|c|}
\hline \multirow{3}{*}{$\frac{\mathrm{C}_{5} \mathrm{H}_{6} \mathrm{~N}_{2}}{\text { Bond }}$} & \multicolumn{2}{|c|}{$\begin{array}{l}\text { 3-Pyridinamine } \\
\text { (3-Aminopyridine) }\end{array}$} & \multirow[b]{2}{*}{$\mathrm{C}_{1}$} \\
\hline & $\mathrm{CH}-\mathrm{CH}-\mathrm{CH}$ & $\mathrm{H}-\mathrm{C}-\mathrm{NII}$ & \\
\hline & Substitution & Angle & Effective \\
\hline $\mathrm{H} \cdots \mathrm{H}^{\prime}$ & $1.672 \mathrm{E}$ & $\begin{array}{l}\mathrm{HNH} \\
\phi\end{array}$ & $\begin{array}{r}113.4 . \mathrm{E} \\
37.0 \mathrm{X}\end{array}$ \\
\hline
\end{tabular}

The parameters for the amine group are obtained from the dewterium substitution along with the assumption that $\mathrm{d}(\mathrm{NH})=1.00$ $A$ and some assumptions for ring parameters. $\phi$ is the angle between the $\mathrm{HNH}$ bisector and the $\mathrm{CN}$ bond.

[1] D. Christen, D. Norbury, D. G. Lister and P. Palmieri, J.C.S., Faraday II, 1975, 438.

\section{4-Pyridinamine \\ (4-Aminopyridine)}

\begin{tabular}{cc|cc}
$\mathrm{C}_{5} \mathrm{H}_{6} \mathrm{~N}_{2}$ & $\mathrm{CH}-\mathrm{CH}-\mathrm{N}-\mathrm{CH}-\mathrm{CH}-\mathrm{C}-\mathrm{NH}_{2}$ & $\mathrm{C}_{\mathrm{B}}$ \\
\hline Bond & Substitution & Angle & Effective \\
\hline $\mathrm{H} \cdots \mathrm{H}^{\prime}$ & $1.699 \mathrm{E}$ & $\begin{array}{l}\mathrm{HNH} \\
\phi\end{array}$ & $\begin{array}{r}116.3 \mathrm{E} \\
27.6 \mathrm{X}\end{array}$ \\
\hline
\end{tabular}

The parameters for the amine group are obtained from deuterium substitution data along with the assumption that $\mathrm{d}(\mathrm{NH})=1.00 \mathrm{~A}$ and some assumptions for ring parameters. $\phi$ is the angle between the HNH bisector and the CN bond.

[1] D. Christen, D. Norbury, D. G. Lister and P. Palmieri, J.C.S., Faraday II, 1975, 438 .

\section{Cyclopent-2-en-1-one}

$\mathrm{C}_{5} \mathrm{H}_{6} \mathrm{O}$

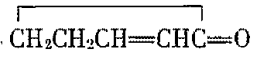

$\mathrm{C}_{4}$

Planarity of heavy atoms has been conclusively established.

[1] D. Chadwick, A. C. Legon and D. J. Millen, Chem. Comm. 1969,1130 (1969).

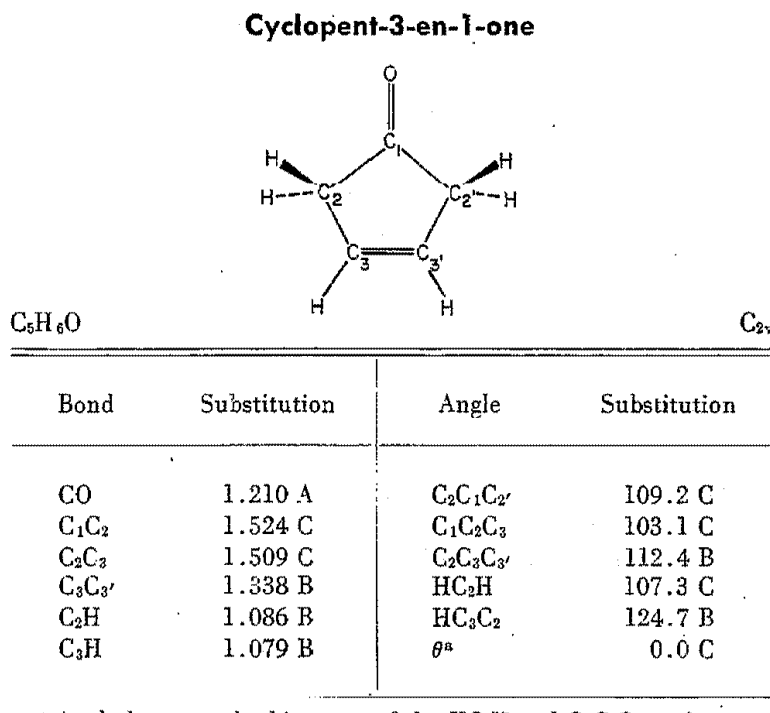

A Angle between the bisectors of the $\mathrm{HC}_{2} \mathrm{H}$ and $\mathrm{C}_{1} \mathrm{C}_{2} \mathrm{C}_{3}$ angles.

[1] J.W. Bevan and A. C. Legon, J. Chem. Soc. Faraday Trans., $67,902(1973)$.

\section{2-Methylfuran}

$\mathrm{C}_{5} \mathrm{H}_{6} \mathrm{O}$

$\mathrm{C}_{s}$

Heavy atom planarity seems assured by the data. See structure of furan.

[1] U. Andersen and H. Dreizler, Z. Naturforsch. 25a, 570 (1970). [2] W. G. Norris and L. C. Krisher, J. Chem. Phys. 51, 403 (1969). 
1-Chlorobicyclo[1.1.1] Pentane

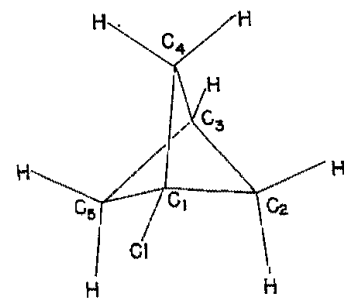

$\mathrm{C}_{5} \mathrm{H}_{7} \mathrm{Cl}$

\begin{tabular}{ll|lr}
\hline \hline Bond & Effective & Angle & Effective \\
\hline & & & \\
$\mathrm{C}_{1} \mathrm{C}_{2}$ & $1.536 \mathrm{X}$ & $\mathrm{C}_{1} \mathrm{C}_{2} \mathrm{C}_{3}$ & $73.5 \mathrm{X}$ \\
$\mathrm{C}_{2} \mathrm{C}_{3}$ & $1.556 \mathrm{X}$ & $\mathrm{C}_{2} \mathrm{C}_{1} \mathrm{C}_{4}$ & $88.6 \mathrm{X}$ \\
$\mathrm{C}_{1} \mathrm{Cl}$ & $1.761 \mathrm{X}$ & $\mathrm{C}_{2} \mathrm{C}_{3} \mathrm{C}_{4}$ & $87.2 \mathrm{X}$ \\
& & $\mathrm{ClC}_{1} \mathrm{C}_{2}$ & $126.2 \mathrm{X}$ \\
& & & \\
\hline
\end{tabular}

In order to obtain the structural parameters, the constrain $r\left(\mathrm{C}_{2} \mathrm{C}_{3}\right)-r\left(\mathrm{C}_{1} \mathrm{C}_{2}\right)=0.02 \pm 0.01 \AA$ and parameters for the methylene groups had to be assumed.

[1] K. W. Cox and M. D. Harmony, J. Mol. Spectrose. 36, 34 (1970).

\section{Cyclobutanecarbonitrile}

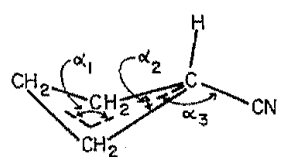

$\mathrm{C}_{5} \mathrm{H}_{7} \mathrm{~N}$

\begin{tabular}{cr}
$\mathrm{C}_{5} \mathrm{H}_{7} \mathrm{~N}$ & $\mathrm{C}_{\mathrm{s}}$ \\
\hline \hline Angle & Effective \\
\hline$\alpha_{1}$ & $21.4 \mathrm{X}$ \\
$\kappa_{2}$ & $90.0 \mathrm{X}$ \\
$\alpha_{3}$ & $133.0 \mathrm{X}$ \\
\end{tabular}

The equatorial conformation was established.

[1] M. Y. Fong and M. D. Harmony, J. Chem. Phys. 58, 4260 (1973).

$\mathrm{C}_{\mathrm{a}}$

\section{Bicyclo[2.1.0]pentane}

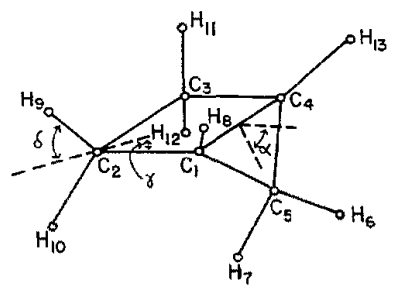

$\mathrm{C}_{55} \mathrm{H}_{8}$

\begin{tabular}{ll|ll}
\hline \hline Bond & Substitution & Angle & Sulstitution \\
\hline & $1.336 \mathrm{~B}$ & $\mathrm{C}_{1} \mathrm{C}_{2} \mathrm{H}_{9}$ & $113.3 \mathrm{D}$ \\
$\mathrm{C}_{1} \mathrm{C}_{4}$ & $1.565 \mathrm{~B}$ & $\mathrm{C}_{1} \mathrm{C}_{2} \mathrm{H}_{10}$ & $115.2 \mathrm{C}$ \\
$\mathrm{C}_{2} \mathrm{C}_{3}$ & $1.528 \mathrm{C}$ & $\mathrm{C}_{3} \mathrm{C}_{2} \mathrm{H}_{9}$ & $111.9 \mathrm{C}$ \\
$\mathrm{C}_{1} \mathrm{C}_{2}$ & $1.507 \mathrm{~B}$ & $\mathrm{C}_{3} \mathrm{C}_{2} \mathrm{H}_{10}$ & $116.6 \mathrm{D}$ \\
$\mathrm{C}_{1} \mathrm{C}_{5}$ & $1.088 \mathrm{~B}$ & $\mathrm{H}_{6} \mathrm{C}_{3} \mathrm{H}_{7}$ & $116.7 \mathrm{C}$ \\
$\mathrm{C}_{5} \mathrm{H}_{6}$ & $1.090 \mathrm{~B}$ & $\mathrm{H}_{8} \mathrm{C}_{1} \mathrm{C}_{5}$ & $121.2 \mathrm{C}$ \\
$\mathrm{C}_{5} \mathrm{H}_{7}$ & $1.082 \mathrm{~B}$ & $\mathrm{H}_{8} \mathrm{C}_{4} \mathrm{C}_{4}$ & $128.6 \mathrm{C}$ \\
$\mathrm{C}_{1} \mathrm{H}_{8}$ & $1.085 \mathrm{C}$ & $\alpha$ & $67.3 \mathrm{C}$ \\
$\mathrm{C}_{2} \mathrm{H}_{8}$ & $1.097 \mathrm{C}$ & $\beta$ & $61.0 \mathrm{C}$ \\
$\mathrm{C}_{2} \mathrm{H}_{10}$ & & $\beta$ & \\
\hline
\end{tabular}

Redundant parameters: $\mathrm{H}_{6} \mathrm{C}_{5} \mathrm{C}_{1}=114.7(\mathrm{C}), \mathrm{H}_{7} \mathrm{C}_{5} \mathrm{C}_{1}=119.0$ (B), $\mathrm{H}_{8} \mathrm{C}_{8} \mathrm{C}_{2}=126.3(\mathrm{C}), \mathrm{H}_{2} \mathrm{C}_{2} \mathrm{H}_{10}=109.4(\mathrm{C}), \gamma=44.8$ (C), $\delta=57.3$ (D). Angle $\beta$ is the acute angle formed by the intersection of $\mathrm{C}_{5} \mathrm{H}_{6}$ with the bisector of the $\mathrm{C}_{1} \mathrm{C}_{5} \mathrm{C}_{4}$ angle.

[1] S. N. Mathur, M. D. Harmony and R. D. Suenram, J. Chem. Phys. 64, 4340 (1976).

\section{Dimerhylallene}

\begin{tabular}{ll|ll} 
Bond & Effective & Angle & Effective \\
\hline $\mathrm{C}_{1} \mathrm{C}_{(2)}$ & $1.514 \mathrm{X}$ & $\mathrm{C}_{(2)} \mathrm{C}_{1} \mathrm{C}_{(2)}$ & $116.4 \mathrm{X}$
\end{tabular}

Double-bond distances were fixed at the allene value, $1.308 \AA$; methyl group and vinyl hydrogen parameters were taken from dimethyl ketene and allene structures, respectively.

[1] J. Demaison and H. D. Rudolph, J. Mol. Spectrosc. 40, 445 (1971). 
3-Methyl-1-butyne

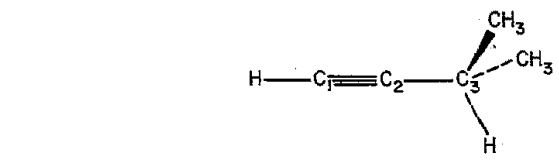

\begin{tabular}{|c|c|c|c|}
\hline Bond & Effective & Angle & Effective \\
\hline $\mathrm{C}_{\mathrm{Me}_{\mathrm{e}} \mathrm{C}_{3}}$ & $1.527 \times$ & $\mathrm{C}_{\mathrm{Me}} \mathrm{C}_{3} \mathrm{C}_{2}$ & $109.6 \mathrm{X}$ \\
\hline $\mathrm{C}_{2} \mathrm{C}_{3}$ & $1.495 \mathrm{X}$ & $\mathrm{C}_{\mathrm{Me}} \mathrm{CC}_{\mathrm{Mo}}$ & $112.9 \times$ \\
\hline $\mathrm{C}_{1} \mathrm{C}_{2}^{\mathrm{a}}$ & $1.203 \wedge$ & $\mathrm{HC}_{1} \mathrm{C}_{2}^{2}$ & 180.0 \\
\hline $\mathrm{C}_{1} \mathrm{H}^{4}$ & $1.058 \mathrm{~A}$ & $\mathrm{HC}_{\mathrm{Ma}_{0}} \mathrm{H}^{\mathrm{a}}$ & 109.5 \\
\hline $\mathrm{C}_{\mathrm{Me}} \mathrm{H}^{\mathrm{A}}$ & 1.092 & & \\
\hline
\end{tabular}

"Assumed value.

[1] A. R. Mochel, A. Biørseth, C. O. Britt, and J. E. Boggs, J. Mol. Spectrose. 48, 107 (1973).

\section{Cyclopentanone}

$\mathrm{C}_{5} \mathrm{H}_{8} \mathrm{O}$

$$
\mathrm{CH}_{2} \mathrm{CH}_{2} \mathrm{CH}_{2} \mathrm{CH}_{2} \mathrm{C}=\mathrm{O}
$$

$\mathrm{C}_{2}$

The ground state conformation is a twisted $\mathrm{C}_{2}$ form.

[1] H. Kim and W. D. Gwinn, J. Chem. Phys: 51, 1815 (1969).

Pivalonitrile

(Tertiary Bulyl Cyanide)

\begin{tabular}{cc|cc}
$\mathrm{C}_{5} \mathrm{H}_{0} \mathrm{~N}$ & $\left(\mathrm{C}^{1} \mathrm{H}_{3}\right)_{2} \mathrm{C}^{2} \mathrm{C}^{3} \mathrm{~N}$ & $\mathrm{C}_{8 *}$ \\
\hline Bond & Substitution & Angle & Substitution \\
\hline $\mathrm{C}^{2}$ & $1.159 \mathrm{~A}$ & $\mathrm{C}^{1} \mathrm{C}^{2} \mathrm{C}^{1}$ & $110.5 \mathrm{C}$ \\
$\mathrm{C}^{1} \mathrm{C}^{2}$ & $1.536 \mathrm{C}$ & & \\
$\mathrm{C}^{2} \mathrm{C}^{3}$ & $1.478 \mathrm{C}$ & & \\
\hline
\end{tabular}

[1] L. J. Nugent, D. E. Mann and D. R. Lide, J. Chem. Phys. 36, $965(1962)$

\section{Cyclopentane}

\begin{tabular}{lrr} 
& $\mathrm{CH}_{2} \mathrm{CH}_{2} \mathrm{CH}_{2} \mathrm{CH}_{2} \mathrm{CH}_{2}$ & Undetermined \\
\hline Bond & Effective \\
\hline $\mathrm{CC}$ & $1.537 \mathrm{X}$
\end{tabular}

$D_{5 \mathrm{~b}}$ symmetry was assumed in the derivation of the above bond distance.

[1] K. Tanner and A. Weber, J. Mol. Spectrosc. 10, 381 (1963).

\section{1,3,5-Trifluorobenzene}

$\mathrm{C}_{6} \mathrm{H}_{3} \mathrm{~F}_{3}$ $\mathrm{D}_{3 \mathrm{~h}}$

\begin{tabular}{cc}
\hline Bond & Effective \\
\hline $\mathrm{CF}$ & $1.304 \mathrm{X}$
\end{tabular}

In order to determine $r_{0}(\mathrm{C}-\mathrm{F})$ the $\mathrm{CC}$ and $\mathrm{CH}$ bond distances were absumed.

[1] J. Schlupf and A. Weber, J. Raman Spectrosc. 1, 3 (1973).

1-Chloro-2-fluorobenzene

(o-Fluorochlorobenzene)

\begin{tabular}{cc}
$\mathrm{C}_{6} \mathrm{H}_{4} \mathrm{ClF}$ & \\
\hline \hline Bond & Effective \\
\hline $\mathrm{CC}$ & $1.397 \mathrm{X}$ \\
$\mathrm{CH}$ & $1.084 \mathrm{X}$ \\
$\mathrm{CF}$ & $1.31 \mathrm{X}$ \\
$\mathrm{CCl}$ & $1.72 \mathrm{X}$ \\
\hline
\end{tabular}

Structure assumes ring is a regular hexagon

[1] P. Kökeritz and H. Selen, Ark. Fys. 30, 193 (1965).

\section{1-Chloro-3-nuorobenzene} (m-Fluorochlorobenzene)

\begin{tabular}{cc}
$\mathrm{C}_{6} \mathrm{H}_{8} \mathrm{ClF}$ & $\mathrm{C}_{\mathrm{s}}$ \\
\hline Bond & Effective \\
\hline $\mathrm{CC}$ & $1.397 \mathrm{X}$ \\
$\mathrm{CH}$ & $1.084 \mathrm{X}$ \\
$\mathrm{CF}$ & $1.329 \mathrm{X}$ \\
$\mathrm{CCl}$ & $1.699 \mathrm{X}$
\end{tabular}

Structure assumes ring is a regular hexagon.

[1] A. Rachman, P. Kökeritz and H. Selen J. Mol. Spectrose. 8, 338 (1962).

\section{m-Difluorobenzene \\ (1,3-Difluorobenzene)}

\begin{tabular}{ll}
$\mathrm{C}_{6} \mathrm{H}_{4} \mathrm{~F}_{2}$ & $\mathrm{C}_{24}$ \\
\hline Bond & Effective \\
\hline $\mathrm{CG}$ & $1.40 \mathrm{X}$ \\
$\mathrm{CH}$ & $1.004 \mathrm{X}$ \\
$\mathrm{CF}$ & $1.30 \mathrm{X}$
\end{tabular}

Ring assumed to be regular hexagon.

[1] L. Nygaard, E. R. Hansen, R. I. Hansen, J. Rastrup-Anỏersen, and G. O. Sorensen, Spectrochim. Acta 23A, 2813 (1967). 


\section{o-Difluorobenzene \\ (1,2-Difluorobenzene)}

$\mathrm{C}_{6} \mathrm{H}_{4} \mathrm{~F}_{2}$

\begin{tabular}{cl}
\hline Bond & Effective \\
\hline $\mathrm{CC}$ & $1.40 \mathrm{X}$ \\
$\mathrm{CH}$ & $1.084 \mathrm{X}$ \\
$\mathrm{CF}$ & $1.31 \mathrm{X}$
\end{tabular}

Ring assumed to be regular hexagon.

[1] A. Hatta, C. Hirose, and K. Kozima, Bull. Chem. Soc. Japan 41, 1088 (1268).

[2] L. Nygaard, E. R. Hansen, R. Lykke. Hansen, J. Rastrup. Andersen and G. O. Sorensen, Spectrochim. Acta 23A, 2813 (1967).

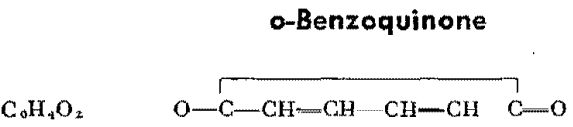

Cov

The observed microwave spectrum supports a planar molecule with the quinonoid structure.

[1] G. L. Blackman, R. D. Brown and A. P. Porter, J. C. S. Chem. Comm. 1975, 499 (1975).

\begin{tabular}{ll}
\multicolumn{2}{c}{ Bromobenzene } \\
$\mathrm{C}_{6} \mathrm{H}_{5} \mathrm{Br}$ & $\mathrm{C}_{2 \mathrm{v}}$ \\
\hline & Effective \\
\hline & \\
$\mathrm{CC}$ & $1.40 \mathrm{X}$ \\
$\mathrm{CH}$ & $1.07 \mathrm{X}$ \\
$\mathrm{CBr}$ & $1.87 \mathrm{X}$
\end{tabular}

Structure assumes regular hexagon. Four isotopic species studier (no $\mathrm{C}^{13}$ species).

[1] E. Rosenthal and B. P. Dailey, J. Chem. Phys. 43, 2093 (1965).

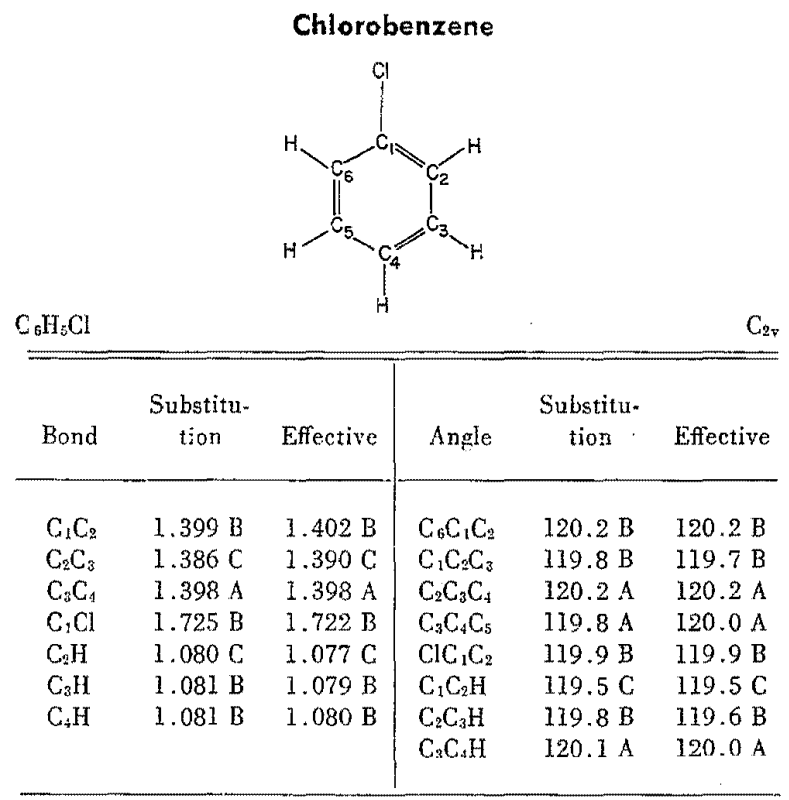

[1] F. Michel, H. Nery, P. Nosberger, and G. Roussy, J. Mol. Struct. 30, 409 (1976).

[2] 6. Rnesey and F. Michel, J. Mol. Struot. 30, 390 (1076).

\section{Fluorobenzene}

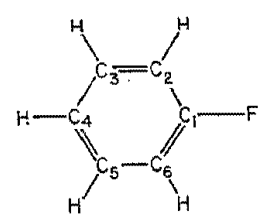

\begin{tabular}{ccc|ccc}
$\mathrm{C}_{6} \mathrm{H}_{5} \mathrm{~F}$ & & & & $\mathrm{C}_{28}$ \\
\hline Bond & $\begin{array}{c}\text { Substitu- } \\
\text { tion }\end{array}$ & Fffective & Angle & $\begin{array}{c}\text { Substitu- } \\
\text { tion }\end{array}$ & Effective \\
& & & & & \\
$\mathrm{C}_{1} \mathrm{C}_{2}$ & & $1.383 \mathrm{D}$ & $\mathrm{C}_{6} \mathrm{C}_{1} \mathrm{C}_{2}$ & & $123.4 \mathrm{D}$ \\
$\mathrm{C}_{2} \mathrm{C}_{3}$ & & $1.395 \mathrm{D}$ & $\mathrm{C}_{1} \mathrm{C}_{2} \mathrm{C}_{3}$ & & $117.9 \mathrm{D}$ \\
$\mathrm{C}_{3} \mathrm{C}_{4}$ & $1.397 \mathrm{~B}$ & & $\mathrm{C}_{2} \mathrm{C}_{3} \mathrm{C}_{4}$ & & $120.5 \mathrm{D}$ \\
$\mathrm{C}_{2} \mathrm{~F}$ & & $1.354 \mathrm{D}$ & $\mathrm{C}_{3} \mathrm{C}_{4} \mathrm{C}_{3}$ & $119.8 \mathrm{~B}$ & \\
$\mathrm{C}_{2} \mathrm{H}$ & & $1.081 \mathrm{D}$ & $\mathrm{C}_{1} \mathrm{C}_{2} \mathrm{H}$ & & $120.0 \mathrm{D}$ \\
$\mathrm{C}_{5} \mathrm{H}$ & $1.083 \mathrm{C}$ & & $\mathrm{C}_{4} \mathrm{C}_{3} \mathrm{H}$ & $119.9 \mathrm{C}$ & \\
$\mathrm{C}_{4} \mathrm{H}$ & $1.080 \mathrm{C}$ & & & & \\
& & & & &
\end{tabular}

[1] B. Bak, D. Christensen, L. Hansen -Nygaard, and E. Tannenbaum, J. Chem. Phys. 26, 134. (1957).

[2] L. Nygaard, I. Bojesen, T. Pederson, and I. Rastrup-Andersen, J. Mol. Struct. 2, 209 (1968). 
lodobenzene

\begin{tabular}{ll}
$\mathrm{C}_{6} \mathrm{H}_{6} \mathrm{I}$ & \\
\hline \hline Bond & Effective \\
\hline $\mathrm{CC}$ & $1.397 \mathrm{X}$ \\
$\mathrm{CH}$ & $1.084 \mathrm{X}$ \\
$\mathrm{Cl}$ & $2.08 \mathrm{X}$
\end{tabular}

Structure assumes ring is a regular hexagon.

[I] K. Johansson, H. Oldeberg and H. Selen, Ark. Fys. 29, 531 (1965).

\begin{tabular}{cc|cc}
\multicolumn{3}{c}{ Nitrosobenzene } & \\
\hline Bond & Effective & Angle & Effective \\
\hline $\mathrm{CN}$ & $1.47 \mathrm{X}$ & $\mathrm{CNO}$ & $116 \mathrm{X}$
\end{tabular}

Ring parameters were taken to be those of benzonitrile and NO distance was chosen to be 1.21 .

[1] Y. Hanyu and J. E. Boggs, J. Chem. Phys. 43, 3454 (1965).

\section{2-Nitrophenol}

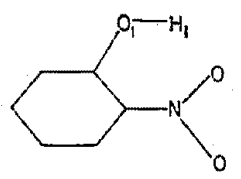

\begin{tabular}{|c|c|}
\hline Bond & Substitution \\
\hline $\mathrm{O}_{1} \mathrm{H}_{\mathrm{r}}$ & $1.00 \mathrm{D}$ \\
\hline
\end{tabular}

[1] S. Leavell and R. F. Curl, Jr., J. Mol. Spectrosc. 45, 4.28 (1973).

Benzene

\begin{tabular}{cc}
$\mathrm{C}_{\mathrm{E}} \mathrm{H}_{6}$ & \\
\hline Bond & Effective \\
\hline $\mathrm{CC}$ & $1.396 \mathrm{~B}$ \\
$\mathrm{CH}$ & $1.083 \mathrm{~B}$
\end{tabular}

[1] A. Cabena, J. Bachand and J. Giguere, Can. J. Phys. 52, 1949 (1974).
5-Methylene-1,3-Cyclopentadiune (Fulvene)

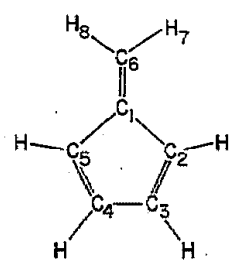

\begin{tabular}{|c|c|c|c|}
\hline Bbond & Substilutive & Angle & Sulstitution \\
\hline $\mathrm{C}_{8} \mathrm{C}_{8}$ & $1.348 \mathrm{~A}$ & $\mathrm{C}_{2} \mathrm{C}_{1} \mathrm{C}_{5}$ & $106.8 \mathrm{~A}$ \\
\hline $\mathrm{C}_{1} \mathrm{C}_{2}$ & $1.468 \mathrm{~A}$ & $\mathrm{C}_{1} \mathrm{C}_{2} \mathrm{C}_{3}$ & $107.7 \mathrm{~A}$ \\
\hline $\mathrm{C}_{2} \mathrm{C}_{3}$ & $1.357 \mathrm{~A}$ & $\mathrm{C}_{2} \mathrm{C}_{3} \mathrm{C}_{4}$ & $108.9 \mathrm{~A}$ \\
\hline $\mathrm{C}_{5} \mathrm{C}_{4}$ & $1.476 \mathrm{~A}$ & $\mathrm{C}_{1} \mathrm{C}_{2} \mathrm{H}$ & $124.9 \mathrm{~A}$ \\
\hline $\mathrm{C}_{2} \mathrm{H}$ & $1.077 \mathrm{~A}$ & $\mathrm{C}_{2} \mathrm{C}_{3} \mathrm{H}$ & $126.4 \mathrm{~A}$ \\
\hline $\mathrm{C}_{3} \mathrm{H}$ & $1.080 \mathrm{~A}$ & $\mathrm{H}_{7} \mathrm{C}_{8} \mathrm{H}_{8}$ & $118.1 \mathrm{~A}$ \\
\hline $\mathrm{C}_{6} \mathrm{H}_{7}$ & $1.083 \mathrm{~A}$ & & \\
\hline
\end{tabular}

[1] P. A. Baron, R. D. Brown, F. R. Burden, P. J. Domaille and J. E. Kent, J. Mol. Speetrose, 43, 401 (1972).

[2] R. D. Suenram and M. D. Harmony, J. Chem. Phys. 58, 5843 (1973).

Tricyclo[3.1.0.02,6]hex-3-ene (Benzvalene)

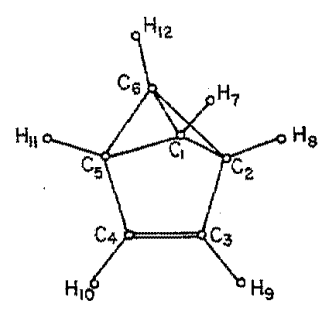

\begin{tabular}{cc|cc}
$\mathrm{C}_{6} \mathrm{H}_{6}$ & & & $\mathrm{C}_{2 \mathrm{v}}$ \\
\hline \hline Bond & Substitution & Angle & Substitution \\
\hline & & & \\
$\mathrm{C}_{2} \mathrm{C}_{6}$ & $1.452 \mathrm{~A}$ & $\alpha^{\mathrm{a}}$ & $106.0 \mathrm{C}$ \\
$\mathrm{C}_{4} \mathrm{C}_{2}$ & $1.529 \mathrm{~B}$ & $\mathrm{C}_{6} \mathrm{C}_{2} \mathrm{H}_{7}$ & $133.7 \mathrm{~A}$ \\
$\mathrm{C}_{2} \mathrm{C}_{3}$ & $1.503 \mathrm{C}$ & $\mathrm{C}_{2} \mathrm{C}_{1} \mathrm{H}_{7}$ & $135.3 \mathrm{~A}$ \\
$\mathrm{C}_{3} \mathrm{C}_{4}$ & $1.339 \mathrm{~A}$ & $\mathrm{C}_{1} \mathrm{C}_{2} \mathrm{H}_{8}$ & $119.8 \mathrm{C}$ \\
$\mathrm{C}_{7} \mathrm{H}_{2}$ & $1.078 \mathrm{~A}$ & $\mathrm{C}_{3} \mathrm{C}_{2} \mathrm{H}_{3}$ & $124.2 \mathrm{C}$ \\
$\mathrm{C}_{4} \mathrm{H}_{8}$ & $1.082 \mathrm{~A}$ & $\mathrm{C}_{2} \mathrm{C}_{3} \mathrm{H}_{3}$ & $125.4 \mathrm{~A}$ \\
$\mathrm{C}_{3} \mathrm{II}_{3}$ & $1.070 \mathrm{~A}$ & $\mathrm{C}_{4} \mathrm{C}_{3} \mathrm{II}_{9}$ & $128.9 \mathrm{~A}$ \\
& & $\mathrm{C}_{2} \mathrm{C}_{3} \mathrm{C}_{4}$ & $105.7 \mathrm{~A}$ \\
& & & \\
\hline
\end{tabular}

s Dihedral angle of four membered ring.

[1] K. D. Suenram and M. D. Harmony, J, Amer. Chem. Soc. 95, $4506(1973)$. 
Phenol<smiles>Oc1ccccc1</smiles>

Aniline<smiles>NC1C=CCC=CC1</smiles>

$\mathrm{C}_{6} \mathrm{H}_{6} \mathrm{O}$

\begin{tabular}{lll|ll}
\hline \hline Bond & Effective & Substitution & Angle & Effective \\
\hline $\mathrm{CC}$ & $1.397 \mathrm{X}$ & & $\mathrm{COH}$ & $109.0 \mathrm{X}$ \\
$\mathrm{C}_{2} \mathrm{H}$ & $1.084 \mathrm{X}$ & & $\mathrm{OC}_{1} \mathrm{C}_{2}$ & $122.2 \mathrm{X}$ \\
$\mathrm{C}_{3} \mathrm{H}$ & $1.076 \mathrm{X}$ & & & \\
$\mathrm{C}_{4} \mathrm{H}$ & $1.082 \mathrm{X}$ & & & \\
$\mathrm{CO}$ & $1.364 \mathrm{X}$ & \multirow{2}{*}{$0.956 \mathrm{X}$} & & \\
$\mathrm{OH}$ & & 0.956 & & \\
\hline
\end{tabular}

Assumed:

Ring is a regular hexagon.

$\mathrm{C}_{2}$ axis of benzene ring coincides with the $b$ principal axis.

Center w Inass of $\mathrm{OH}$ group lies on the $b$ axis.

[1] T. Kojima, J. Phys. Soc. Japan 15, 284 (1960).

[2] H. Forest and B. P. Dailey, J. Chem, Phys. 45, 1736 (1966).

[3] T. Pederson, M. W. Larsen and L. Nygaard, J. Mol. Struct. 4, 50 (1969).

\section{Thiophenol}

$\mathrm{L}_{6} \mathrm{H}_{6} \mathrm{~S}$

$$
\mathrm{C}_{6} \mathrm{H}_{6} \mathrm{SH}
$$

Data are consistent with planarity.

[1] K. I. Johansson, H. Oldeberg and H. Selen, Arkiv. Fysik. 33, 313 (1967).

$\mathrm{C}_{\mathrm{s}}$
$\mathrm{C}_{8} \quad \mathrm{C}_{6} \mathrm{H}_{7} \mathrm{~N}$

\begin{tabular}{ll|ll}
\hline \hline Bond & Substitution & Angle & Substitution \\
\hline & & & \\
$\mathrm{NH}_{1}$ & $1.001 \mathrm{D}$ & $\mathrm{H}_{1} \mathrm{NH}_{1}$ & $113.1 \mathrm{D}$ \\
$\mathrm{C}_{1} \mathrm{~N}$ & $1.402 \mathrm{~B}$ & $\mathrm{C}_{6} \mathrm{C}_{1} \mathrm{C}_{2}$ & $119.4 \mathrm{~A}$ \\
$\mathrm{C}_{1} \mathrm{C}_{2}$ & $1.397 \mathrm{~B}$ & $\mathrm{C}_{1} \mathrm{C}_{2} \mathrm{C}_{3}$ & $120.1 \mathrm{~A}$ \\
$\mathrm{C}_{2} \mathrm{C}_{3}$ & $1.394 \mathrm{~B}$ & $\mathrm{HC}_{2} \mathrm{C}_{3}$ & $120.1 \mathrm{~A}$ \\
$\mathrm{C}_{3} \mathrm{C}_{4}$ & $1.396 \mathrm{~B}$ & $\mathrm{C}_{2} \mathrm{C}_{3} \mathrm{C}_{4}$ & $120.7 \mathrm{~A}$ \\
$\mathrm{C}_{2} \mathrm{H}$ & $1.082 \mathrm{~B}$ & $\mathrm{HC}_{3} \mathrm{C}_{2}$ & $119.4 \mathrm{~A}$ \\
$\mathrm{C}_{3} \mathrm{H}$ & $1.083 \mathrm{~B}$ & $\mathrm{C}_{3} \mathrm{C}_{4} \mathrm{C}_{5}$ & $118.9 \mathrm{~A}$ \\
$\mathrm{C}_{4} \mathrm{H}$ & $1.080 \mathrm{~B}$ & & \\
\hline
\end{tabular}

The $\mathrm{C}_{6} \mathrm{H}_{5} \mathrm{~N}$ fragment is essentially planar. The dihedral angle between the $\mathrm{NH}_{2}$ and $\mathrm{C}_{6} \mathrm{H}_{5} \mathrm{~N}$ planes is $37.55^{\circ}$.

[1] D. G. Lister, J. K. Tyler, J. H. Hog, and N. W. Larsen, J. Mol. Struct, 23, 253 (1974).

[2] D. G. Lister and J. K. Tyler, Chem. Commun. 1966, 152 (1966).

\section{3-Methylenecyclobutanecarbonitrile}

$\mathrm{C}_{6} \mathrm{H}_{7} \mathrm{~N}$

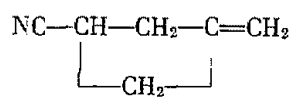

$\mathrm{C}_{\mathrm{B}}$

The ring is non-planar with the CN group in the equatorial position.

[1] J. R. Durig, Y. S. Li, M. D. Harmony and M. Y. Fong, J. Mol. Struct. 23, 377 (1974).

Bicyclo[2.1.1]hex-2-ene

\begin{tabular}{|c|c|c|c|}
\hline Bond & Substitution & Angle & Substitution \\
\hline $\mathrm{C}_{1} \mathrm{C}_{2}$ & $1.528 \mathrm{~B}$ & $\mathrm{C}_{1} \mathrm{C}_{2} \mathrm{C}_{3}$ & $103.3 \mathrm{C}$ \\
\hline $\mathrm{C}_{2} \mathrm{C}_{3}$ & $1.341 \mathrm{~B}$ & $\mathrm{C}_{5} \mathrm{C}_{2} \mathrm{C}_{2}$ & $100.4 \mathrm{C}$ \\
\hline \multirow[t]{3}{*}{$\mathrm{C}_{1} \mathrm{C}_{5}$} & $1.568 \mathrm{~B}$ & $\mathrm{C}_{5} \mathrm{C}_{1} \mathrm{C}_{6}$ & $85.3 \mathrm{C}$ \\
\hline & & $\mathrm{C}_{1} \mathrm{C}_{5} \mathrm{C}_{4}$ & $81.4 \mathrm{C}$ \\
\hline & & $\theta^{\mathrm{a}}$ & $126.7 \mathrm{C}$ \\
\hline
\end{tabular}

${ }^{2}$ Dihedral angle formed by intersection of $\mathrm{C}_{4} \mathrm{C}_{5} \mathrm{C}_{1}$ and $\mathrm{C}_{4} \mathrm{C}_{6} \mathrm{C}_{1}$ planes.

[1] C. S. Wang and M. D. Harmony, J. Am. Chem. Soc. 98, 1976. 


\section{1,3-Cyclohexadiene}

\begin{tabular}{cc}
$\mathrm{C}_{6} \mathrm{H}_{8} \quad$ & $\mathrm{H}_{2} \mathrm{C}-\mathrm{HC}=\mathrm{CH}-\mathrm{CH}=\mathrm{CH}-\mathrm{CH}_{2}$ \\
Angle & Effective \\
\hline$\tau$ & $17.5 \mathrm{X}$
\end{tabular}

$r$ is the toroional angle botwoon the two double boride. [1] S. S. Butcher, J. Chem. Phys, 42, 1830 (1965).

Tricyclo[2.2.0.0 2,6$]$ hexane

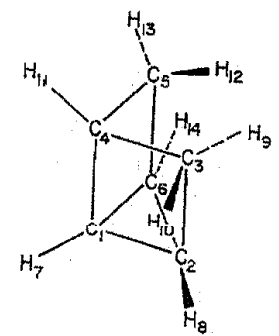

$\mathrm{C}_{6} \mathrm{H}_{8}$

\begin{tabular}{|c|c|c|c|c|c|}
\hline Bond & $\begin{array}{l}\text { Substitu- } \\
\text { tion }\end{array}$ & Effective & Angle & $\begin{array}{l}\text { Substitu- } \\
\text { tion }\end{array}$ & Effective \\
\hline $\mathrm{C}_{1} \mathrm{C}_{2}$ & $1.513 \mathrm{~B}$ & $1.518 \mathrm{C}$ & $\mathrm{H}_{9} \mathrm{C}_{3} \mathrm{H}_{10}$ & $110.2 \mathrm{C}$ & $110.7 \mathrm{C}$ \\
\hline $\mathrm{C}_{1} \mathrm{C}_{4}$ & $1.584 \mathrm{~B}$ & $1.589 \mathrm{C}$ & $\mathrm{C}_{3} \mathrm{C}_{4} \mathrm{H}_{31}$ & $126.4 \mathrm{~B}$ & $126.5 \mathrm{~B}$ \\
\hline $\mathrm{C}_{2} \mathrm{C}_{3}$ & $1.523 \mathrm{~B}$ & $1.527 \mathrm{~B}$ & $\mathrm{C}_{3} \mathrm{C}_{4} \mathrm{H}_{17}$. & $120.9 \mathrm{~B}$ & $120.9 \mathrm{~B}$ \\
\hline $\mathrm{C}_{2} \mathrm{C}_{6}$ & 1.533 .8 & $1.541 \mathrm{C}$ & $\mathrm{C}_{4} \mathrm{C}_{1} \mathrm{H}_{7}$ & $134.6 \mathrm{~B}$ & $134.5 \mathrm{~B}$ \\
\hline $\mathrm{C}_{3} \mathrm{C}_{4}$ & $1.54 .9 \mathrm{~B}$ & $1.553 \mathrm{~B}$ & $\mathrm{C}_{2} \mathrm{C}_{1} \mathrm{H}_{7}$ & $131.9 \mathrm{~B}$ & $131.8 \mathrm{~B}$ \\
\hline $\mathrm{C}_{7} \mathrm{II}_{7}$ & $1.079 \mathrm{C}$ & $1.077 \mathrm{G}$ & $\mathrm{C}_{1} \mathrm{C}_{2} \mathrm{II}_{8}$ & $127.4 \mathrm{~J}$ & $122.4 \mathrm{~B}$ \\
\hline $\mathrm{C}_{2} \mathrm{H}_{\theta}$ & $1.082 \mathrm{C}$ & $1.080 \mathrm{C}$ & $\mathrm{C}_{6} \mathrm{C}_{2} \mathrm{H}_{3}$ & $120.6 \mathrm{~B}$ & $120.5 \mathrm{~B}$ \\
\hline $\mathrm{C}_{3} \mathrm{H}_{9}$ & $1.099 \mathrm{C}$ & $1.098 \mathrm{C}$ & $\mathrm{C}_{3} \mathrm{C}_{5} \mathrm{H}_{8}$ & $128.4 \mathrm{~B}$ & $128.6 \mathrm{~B}$ \\
\hline $\mathrm{C}_{3} \mathrm{H}_{10}$ & $1.087 \mathrm{C}$ & $1.090 \mathrm{C}$ & $\mathrm{C}_{1} \mathrm{C}_{2} \mathrm{C}_{5}$ & $91.8 \mathrm{~B}$ & \\
\hline \multirow[t]{5}{*}{$\mathrm{C}_{4} \mathrm{H}_{41}$} & $1.086 \mathrm{C}$ & $1.086 \mathrm{C}$ & $\mathrm{C}_{2} \mathrm{C}_{3} \mathrm{C}_{4}$ & $86.2 \mathrm{~A}$ & \\
\hline & & & $\mathrm{C}_{3} \mathrm{C}_{4} \mathrm{C}_{2}$ & $88.2 \mathrm{~A}$ & \\
\hline & & & $\mathrm{C}_{4} \mathrm{C}_{1} \mathrm{C}_{2}$ & $85.4 \mathrm{~A}$ & \\
\hline & & & $\mathrm{C}_{1} \mathrm{C}_{2} \mathrm{C}_{5}$ & $59.6 \Lambda$ & \\
\hline & & & $\mathrm{C}_{2} \mathrm{C}_{3} \mathrm{C}_{6}$ & $60.9 \Lambda$ & \\
\hline
\end{tabular}

[1] R. D. Suenram, J. Amer, Chem. Soc. 97, 4869 (1975).

1-Chloro-3,3-dimethyl-1-butyne (Tertiary Butyl Chloroacetylene)

\begin{tabular}{ccc}
$\mathrm{C}_{0} \mathrm{H}_{3} \mathrm{Cl}$ & $\left(\mathrm{CH}_{3}\right)_{3} \mathrm{C}^{1} \mathrm{C}^{2} \equiv \mathrm{C}^{3} \mathrm{Cl}$ & $\mathrm{C}_{30}$ \\
\hline Bond & Substitution & Effective \\
\hline $\mathrm{C}^{3} \mathrm{Cl}$ & $1.638 \mathrm{~B}$ & \\
$\mathrm{C}^{1} \cdots \mathrm{C}^{2}$ & $2.671 \mathrm{~B}$ & \\
$\mathrm{C}^{1} \mathrm{C}^{2 \mathrm{~s}}$ & $1.47 \mathrm{X}$ & $1.47 \mathrm{D}$ \\
\hline
\end{tabular}

${ }^{8} r\left(\mathrm{C}^{1} \mathrm{C}^{2}\right)$ calculated assuming $r\left(\mathrm{C}^{2} \mathrm{C}^{3}\right)$ is $1.205 \AA$.

[1] H. Bodenseh, R. Gegenheimer, J. Mennicke and W. Zeil, Z. Naturforsch. 22A, 523 (1967).

\section{Cyclohexene}

$\mathrm{C}_{6} \mathrm{H}_{10}$

Stark effect data and rotational constants are consistent with "half-chair" form ( $C_{2}$ symmetry) for the molecule. Data are insufficient for structure determination.

[1] L. H. Scharpen, J. E. Wollrab, and D. P. Ames, J. Chem. Phys. 49, 2368 (1968).

\section{3,3-Dimethyl-1-butyne \\ ( $t$-Butyl Acetylene)}

\begin{tabular}{ll|cc}
$\mathrm{C}_{\mathrm{b}} \mathrm{I}_{\mathrm{w}}$ & $\left(\mathrm{C}^{(2)} \mathrm{H}_{3}\right)_{3} \mathrm{C}^{(3)}-\mathrm{C}^{(2)}=\mathrm{C}^{(1)} \mathrm{II}$ & $\mathrm{C}_{3 \mathrm{r}}$ \\
\hline Bond & Substitation & Angle & Substitution \\
& $1.056 \mathrm{~A}$ & $\mathrm{C}^{(2)} \mathrm{C}^{(3)} \mathrm{C}^{(4)}$ & $108.0 \mathrm{C}$ \\
$\mathrm{C}^{(1)} \mathrm{H}$ & $1.209 \mathrm{~A}$ & & \\
$\mathrm{C}^{(1)} \mathrm{C}^{(2)}$ & $1.496 \mathrm{D}$ & & \\
$\mathrm{C}^{(2)} \mathrm{C}^{(3)}$ & $1.532 \mathrm{C}$ & & \\
$\mathrm{C}^{(3)} \mathrm{C}^{(4)}$ & & \\
\hline
\end{tabular}

[1- L. J. Nugent, D. E. Mann and D. R. I.ide, Jr., J. Chem. Phys. 36,965 (1962)

$\mathrm{C}_{\mathrm{s}}$

\section{1,1-Difluorocyclohexane}

$\mathrm{C}_{\mathrm{s}}$

The microwave data show that the molecule adopts the chair conformation.

[1] D. Damiani and L. Ferretti, Chem. Phys. Lett. 24, 357 (1974).

\section{Cyclohexanone}

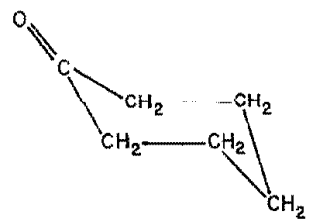

$\mathrm{C}_{6} \mathrm{H}_{10} \mathrm{O}$

$\mathrm{C}_{\text {。 }}$

Rotational constants of assigned transitions are for the "chair" form.

[1] Y. Ohnishi and K. Kozima, Bull. Chem. Soe. Japan 41, 1323 (1968). 
7-Oxabicyclo[2.2.1]heptane

\begin{tabular}{|c|c|c|c|c|}
\hline $\mathrm{C}_{6} \mathrm{H}_{10} \mathrm{O}$ & & & & $\mathrm{C}_{2}$ \\
\hline Bond & Substitution & Effective & Angle & Substitution \\
\hline $\mathrm{C}_{1} \mathrm{C}_{2}$ & $1.537 \mathrm{~B}$ & & $\mathrm{C}_{1} \mathrm{C}_{2} \mathrm{C}_{3}$ & $101.2 \mathrm{~B}$ \\
\hline $\mathrm{C}_{2} \mathrm{C}_{3}$ & $1.551 \mathrm{~B}$ & & $\mathrm{C}_{2} \mathrm{C}_{1} \mathrm{C}_{8}$ & $109.9 \mathrm{~B}$ \\
\hline $\mathrm{C}_{1} \mathrm{O}$ & & $1.452 \mathrm{C}$ & & \\
\hline
\end{tabular}

Hydrogen parameters were assumed to obtain the CO distance. [1] R. A. Creswell, J. Mol. Spectrosc. 56, 133 (1975).

\section{Fluorocyclohexane}

\section{$\mathrm{C}_{6} \mathrm{H}_{11} \mathrm{~F}$}

Insufficient data available for structure determination. Both axial and equatorial conformers observed. Ground vibrational state of equatorial form is more stable by $259 \pm 28 \mathrm{cal} / \mathrm{mol}$.

[1] L. H. Scharpen, J. Amer. Chem. Soc. 94, 3737 (1972).

\section{Cyclohexane}

\begin{tabular}{cccc}
$\mathrm{C}_{6} \mathrm{H}_{12}$ & $\stackrel{\mathrm{CH}_{2} \mathrm{CH}_{2} \mathrm{CH}_{2} \mathrm{CH}_{2} \mathrm{CH}_{2} \mathrm{CH}_{2}}{\mathrm{D}_{3 \mathrm{~d}}}$ \\
\hline Bond & Effective & Angle & Effective \\
\hline $\mathrm{CC}$ & $1.535 \mathrm{X}$ & $\mathrm{HCH}$ & $110.0 \mathrm{X}$ \\
\hline
\end{tabular}

Conformation is the chair form.

[1] R. A. Peters, W. J. Walker and A. Weher, J. Raman Spectrosc. I, 159 (1973).

\section{Hexafluorobenzene}

$\frac{\mathrm{C}_{6} \mathrm{~F}_{6}}{\text { Bond }}=\frac{\text { Effective }}{\mathrm{CF}}$

In order to obtain the $C F$ bond distance it was necessary to as. sume the CC distance.

[1] J. Schlupf and A. Weber, J. Raman. Spectrosc. 1, 3 (1973).

\section{Benzonitrile}

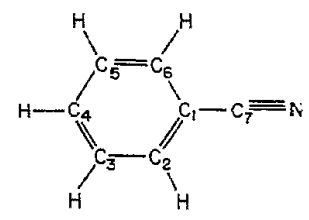

\begin{tabular}{|c|c|c|c|}
\hline Bond & Substitution & Angle & Substitution \\
\hline $\mathrm{C}_{1} \mathrm{Cls}_{\mathrm{B}}$ & 1. $388 \mathrm{C}$ & $\mathrm{C}_{0} \mathrm{C}_{1} \mathrm{C}_{m}$ & $121.8 \mathrm{C}$ \\
\hline $\mathrm{C}_{2} \mathrm{C}_{3}$ & $1.396 \mathrm{C}$ & $\mathrm{C}_{1} \mathrm{C}_{2} \mathrm{C}_{3}$ & $119.0 \mathrm{~B}$ \\
\hline $\mathrm{C}_{3} \mathrm{C}_{4}$ & $1.397 \mathrm{~B}$ & $\mathrm{C}_{2} \mathrm{C}_{3} \mathrm{C}_{4}$ & $120.1 \mathrm{~A}$ \\
\hline $\mathrm{C}_{1} \mathrm{C}_{\bar{z}}$ & $1.451 \mathrm{~B}$ & $\mathrm{C}_{3} \mathrm{C}_{4} \mathrm{C}_{3}$ & $120.1 \mathrm{~A}$ \\
\hline $\mathrm{C}_{7} \mathrm{~N}$ & $1.158 \mathrm{~A}$ & $\mathrm{C}_{3} \mathrm{C}_{2} \mathrm{H}$ & $120.4 \mathrm{C}$ \\
\hline $\mathrm{C} H$ & $1.080 \mathrm{C}$ & $\mathrm{C}_{2} \mathrm{C}_{3} \mathrm{H}$ & $120.0 \mathrm{~A}$ \\
\hline $\mathrm{C}_{3} \mathrm{H}$ & $1.082 \mathrm{~B}$ & & \\
\hline $\mathrm{C}_{4} \mathrm{H}$ & $1.080 \mathrm{~A}$ & & \\
\hline
\end{tabular}

[1] J. Casado, L. Nygaard, and G. O. Sqrensen, J. Mol. Struct. 8, 211 (1971).

[2] B. Bak, D. Christensen, W. B. Dixon, L. Hansen-Nygaard, and I. Rastrup-Andersen, I. Chem. Phyc. 37, 2097 (1062).

\section{Isocyanatobenzene \\ (Phenylisocyanate)}

$\mathrm{C}_{7} \mathrm{H}_{5} \mathrm{NO}$

$\mathrm{C}_{6} \mathrm{H}_{3} \mathrm{NCO}$

$\mathrm{C}_{\mathrm{R}}$

The microwave data indicate a planar structure with a non-linear $\mathrm{C}-\mathrm{N}=\mathrm{C}=\mathrm{O}$ moiety.

[1] A. Bouchy and G. Roussy, C. R. Acad. Sci. Paris 277, 1.43 (1973).

\section{Salicylaldehyde}

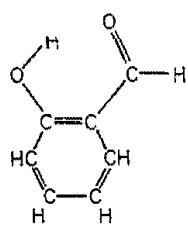

$\mathrm{C}_{7} \mathrm{H}_{6} \mathrm{O}_{2}$ $\mathrm{C}_{\mathrm{s}}$

\begin{tabular}{c|c}
\hline Bond & Substitution \\
\hline $0 \ldots 11$ & $1.76 \mathrm{C}$ \\
\hline
\end{tabular}

[1] H. Jones and R. F. Curl, Jr., J. Mol. Spectrosc. 42, 65 (1972), 
4-Chloro-tricycio[2.2.1.02,6] heptane

(4-Chloronoriticyclene)

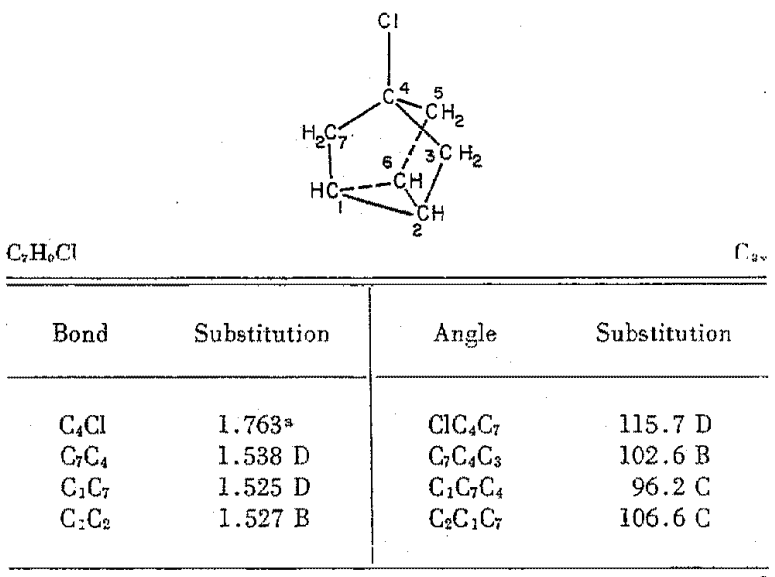

- This distance was assumed with an uncertainty of \pm 0.003 . in order to determine the $\mathrm{C}_{4}$ substitution coordinate.

[1. V. W. Laurie and W. M. Stiglianf, J. Am. Chem. Soc. 95, 4154 (1973).

\section{Ethynyl benzene \\ (Phenyl acetylene)}

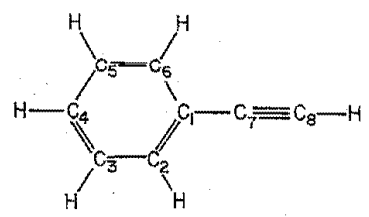

$\mathrm{C}_{8} \mathrm{H}_{6}$

\begin{tabular}{|c|c|c|c|c|c|}
\hline Bond & $\begin{array}{l}\text { Subetitu } \\
\text { tion }\end{array}$ & Effective & Angle & $\begin{array}{c}\text { Subotitu } \\
\text { tion }\end{array}$ & Effective \\
\hline $\begin{array}{l}\mathrm{C}_{4} \mathrm{C}_{8} \\
\mathrm{C}_{2} \mathrm{C}_{3} \\
\mathrm{C}_{3} \mathrm{C}_{4} \\
\mathrm{C}_{1} \mathrm{C}_{7} \\
\mathrm{C}_{7} \mathrm{C}_{8} \\
\mathrm{C}_{8} \mathrm{H}\end{array}$ & $\begin{array}{l}1.398 \mathrm{~A} \\
1.208 \mathrm{~A} \\
1.055 \mathrm{~A}\end{array}$ & $\begin{array}{l}1.388 \mathrm{C} \\
1.396 \mathrm{D} \\
1.448 \mathrm{C}\end{array}$ & $\begin{array}{l}\mathrm{C}_{2} \mathrm{C}_{1} \mathrm{C}_{6} \\
\mathrm{C}_{1} \mathrm{C}_{2} \mathrm{C}_{3} \\
\mathrm{C}_{2} \mathrm{C}_{3} \mathrm{C}_{4} \\
\mathrm{C}_{3} \mathrm{C}_{4} \mathrm{C}_{5}\end{array}$ & $119.9 \mathrm{~A}$ & $\begin{array}{l}120.8 \mathrm{C} \\
119.8 \mathrm{~B} \\
119.9 \mathrm{~B}\end{array}$ \\
\hline
\end{tabular}

Ring $\mathrm{CH}$ parameters were assumed for calculation of effective bond distances and angles.

[1] A. P. Cox, I. C. Ewart, and W. M. Stigliani, J. Chem. Soc. Faraday Trans. II. 71, 504 (1975).
7.Methylenem 1,3,3-cyclohoplatriuns: (Heptertulvens)

$\mathrm{C}_{8} \mathrm{H}_{5}$

$\therefore$

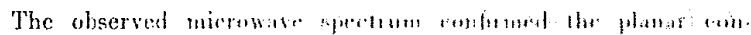
formation.

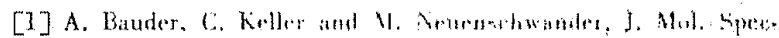
trose: 6.3. 2931 ! 190101.

ortho-Xylene<smiles>CC1CC=CC(C(C)(C)C)C1C</smiles>

$\mathrm{Cs}_{\mathrm{B}} \mathrm{H}_{10}$

\begin{tabular}{|c|c|c|c|c|c|}
\hline Bond & $\begin{array}{c}\text { Substitu- } \\
\text { tion }\end{array}$ & Effective & Angle & $\begin{array}{c}\text { Substitu } \\
\text { tion }\end{array}$ & Effective \\
\hline $\mathrm{C}_{4} \mathrm{H}_{1}$ & $1.080 \mathrm{~B}$ & & $\mathrm{H}_{1} \mathrm{C}_{4} \mathrm{H}_{2}$ & $108.9 \mathrm{C}$ & \\
\hline $\mathrm{C}_{4} \mathrm{H}_{2}$ & $1.095 \mathrm{~B}$ & & $\mathrm{H}_{2} \mathrm{C}_{4} \mathrm{H}_{3}$ & $106.0 \mathrm{D}$ & \\
\hline $\mathrm{C}_{1} \mathrm{C}_{4}$ & & $1.509 \times$ & $\mathrm{C}_{4} \mathrm{C}_{1} \mathrm{C}_{2^{\prime}}$ & & $121.1 \mathrm{X}$ \\
\hline $\mathrm{C}_{1} \mathrm{C}_{2}$ & & $1.414 X$ & $\mathrm{C}_{2} \mathrm{C}_{1} \mathrm{C}_{1^{\prime}}$ & & $119.8 \times$ \\
\hline $\mathrm{C}_{2} \mathrm{C}_{1^{\prime}}$ & & $1.394 \mathrm{X}$ & $\mathrm{C}_{1} \mathrm{C}_{2} \mathrm{H}$ & & $118.9 \mathrm{X}$ \\
\hline $\mathrm{C}_{2} \mathrm{H}$ & & $1.072 \mathrm{X}$ & $\mathrm{C}_{2} \mathrm{C}_{3} \mathrm{H}$ & & $119.7 \mathrm{X}$ \\
\hline \multirow[t]{2}{*}{$\mathrm{C}_{3} \mathrm{H}$} & & $1.079 \times$ & $\mathrm{H}_{1} \mathrm{C}_{4} \mathrm{C}_{1}$ & & $111.2 \mathrm{X}$ \\
\hline & & & $\mathrm{H}_{2} \mathrm{C}_{4} \mathrm{C}_{1}$ & & $111.0 \mathrm{X}$ \\
\hline
\end{tabular}

[1] H. D. Rudolph, K. Walzer and I. Krutzik, J. Mol. Spectrosc. $47,314 .(1973)$.

1-Cyanoadamantane

\begin{tabular}{|c|c|c|c|c|}
\hline$a_{1} \mathrm{H}_{i j, \mathrm{~N}} \mathrm{~N}$ & & $\mathrm{C}_{10} \mathrm{H}_{10} \mathrm{CN}$ & & $\mathrm{C}_{3 v}$ \\
\hline Bond & Substitution & Effective & Angle & Effective \\
\hline $\mathrm{CN}$ & $1.159 \mathrm{~A}$ & & $\mathrm{CCC}$ & $109.5^{\mathrm{a}}$ \\
\hline $\mathrm{CC}$ & & $1.543 \mathrm{X}$ & $\mathrm{HCH}$ & $109.5^{\mathrm{a}}$ \\
\hline $\mathrm{CC}(\mathrm{N})$ & & $1.466 \mathrm{X}$ & & \\
\hline $\mathrm{CH}$ & & $1.09^{\circ}$ & . & \\
\hline
\end{tabular}

All CC bonds were assumed to be equal.

a Assumed values.

[1] D. Chadwick, A. C. Legon and D. J. Millen, J. Chem. Soc. Faraday Trans. 68, 2064 (1972). 